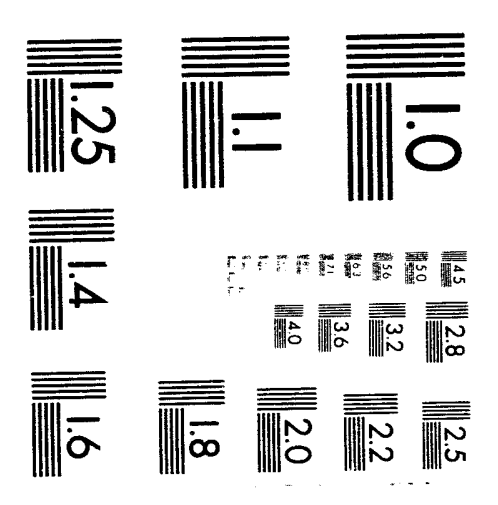



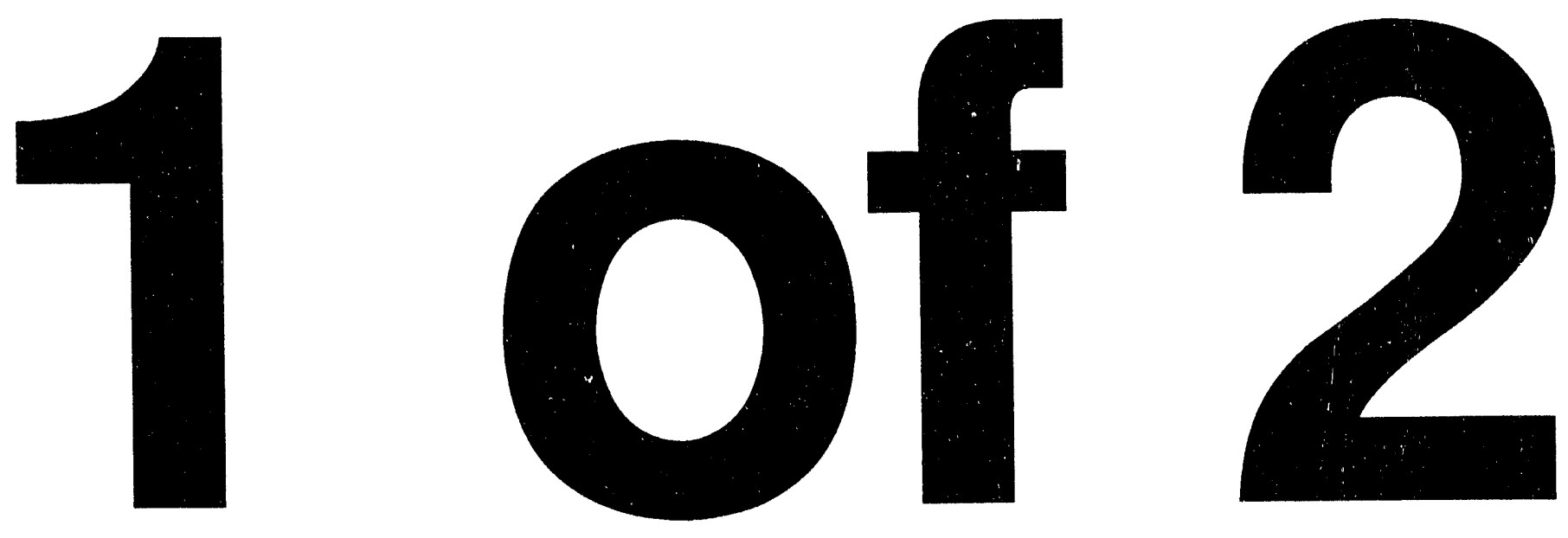


\section{Natural Gas}

\section{Imports and Exports}

\section{Fourth Quarter Report 1993}

Prepared By:

U.S. Department of Energy Office of Fuels Programs Office of Fossil Energy Washington, DC 20585

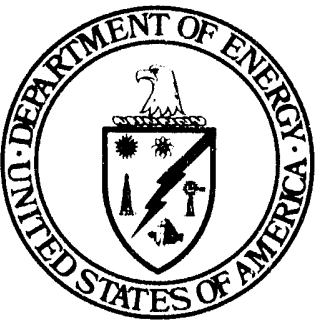




\section{SUMMARY}

The Office of Fuels Programs prepares quarterly reports summarizing the data provided by companies authorized to import or export natural gas. Companies are required, as a condition of their authorizations, to file quarterly reports with the OFP. This report is for the fourth quarter of 1993 (October-December).

Attachment A shows the percentage of takes to maximum firm contract levels and the weighted average per unit price for each of the long-term importers during the 5 most recent reporting quarters.

Attachment B shows volumes and prices of gas purchased by long-term importers and exporters during the past 12 months.

Attachment $C$ shows volume and price information for gas imported on a short-term basis.

Attachment $D$ shows the gas exported on a short-term basis to Canada and Mexico.

During 1993, data indicates gas imports grew by about 10 percent over the 1992 level (2328 vs. 2122 Bcf), with Canadian and Algerian imports increasing by 8 and 82 percent, respectively. During the same time period, exports declined by 41 percent (144 vs. 243 Bcf). Exports to Canada decreased 47 percent from the 1992 level (50 vs. $95 \mathrm{Bcf}$ ) and exports to Mexico decreased by 60 percent ( 38 vs. 95 Bcf).

Highlights to and events affecting importexport activity during the fourth quarter include the following. The PG\&E/PGT pipeline expansion project and 2. new pipelines, Empire State and North Country, became operational. Beginning in November, the majority of Canadian gas marketed in California was imported under short-term authorizations. The number of shortterm importers grew to 109 from 74 during the preceding quarter. The number of active longterm import contracts increased to 202 compared to 157 during the last quarter (36 of these new contracts are attributable to FERC Order No. 636). In December, Mexico began exporting gas to the U.S. for the first time in over 9 years.
Long-term immerts for the quarter totaled 369.5 Bcf, or a 3 percent increase over the fourth quarter of 1992. Long-term Canadian imports totaled about $349.7 \mathrm{Bct}$. The average price of these supplies was $\$ 2.14 / \mathrm{MMBtu}$, which was 2 cents more than the preceding quarter. Under other long-term import arrangements, Distrigas Corporation imported 15.2 Bcf of Algerian LNG at an average landed price of $\$ 2.18 / \mathrm{MMBtu}$ and Pan National Gas Sales, Inc. imported 4.6 Bcf of Algerian LNG at an average tailgate price of \$1.75/MMBtu.

During the fourth quarter, 109 companies used short-term "blanket" authorizations to import $251 \mathrm{Bcf}$ of natural gas. This volume represents a 27 percent increase over the fourth quarter of 1992. Approximately 1.7 Bcf was imported from Mexico at Hidalgo, Texas, at \$1.91/MMBtu.

The average price of Canadian natural gas under short-term import arrangements was $\$ 1.96 / \mathrm{MMBtu}$, compared with $\$ 1.67 / \mathrm{MMBtu}$ during the preceding quarter. Approximately 30 percent of the short-term sales were made at Eastport, Idaho, at an average price of $\$ 1.97 / \mathrm{MMBtu} ; 26$ percent at Sumas, Washington, at $\$ 1.90 / \mathrm{MMBtu} ; 18$ percent at Port of Morgan, Montana, at $\$ 1.70 / \mathrm{MMBtu} ; 12$ percent at Noyes, Minnesota, at \$2.18/MMBtu; 6 percent at Niagara Falls, New York, at \$2.22/MMBtu; 3 percent at Waddington, New York, at $\$ 2.28 / \mathrm{MMBtu}$; and 5 percent at various other entry points, at $\$ 2.04 / \mathrm{MMBiu}$.

In addition, a total of 18 short-term blanket export authorizations were used, exporting a total of 13.4 Bcf of gas. 12 authorizations were used to export about $8.8 \mathrm{Bcf}$ of gas to Canada, at an average price of $\$ 2.46 / \mathrm{MMBtu}$. Under 6 other authorizations, about 4.6 Bcf of gas was exported to Mexico at an average price of $\$ 2.05 / \mathrm{MMB} t u$.

All data are derived from reports filed by importers and exporters, and are subject to future revision. Future revisions to the report will be available on OFP's Electronic Bulletin Board at (202) 586-7853. All general queries concerning this report should be made io Yvonne Caudillo at (202) 586*4587. 
1993 NATURAL GAS TRADE IN REVIEW

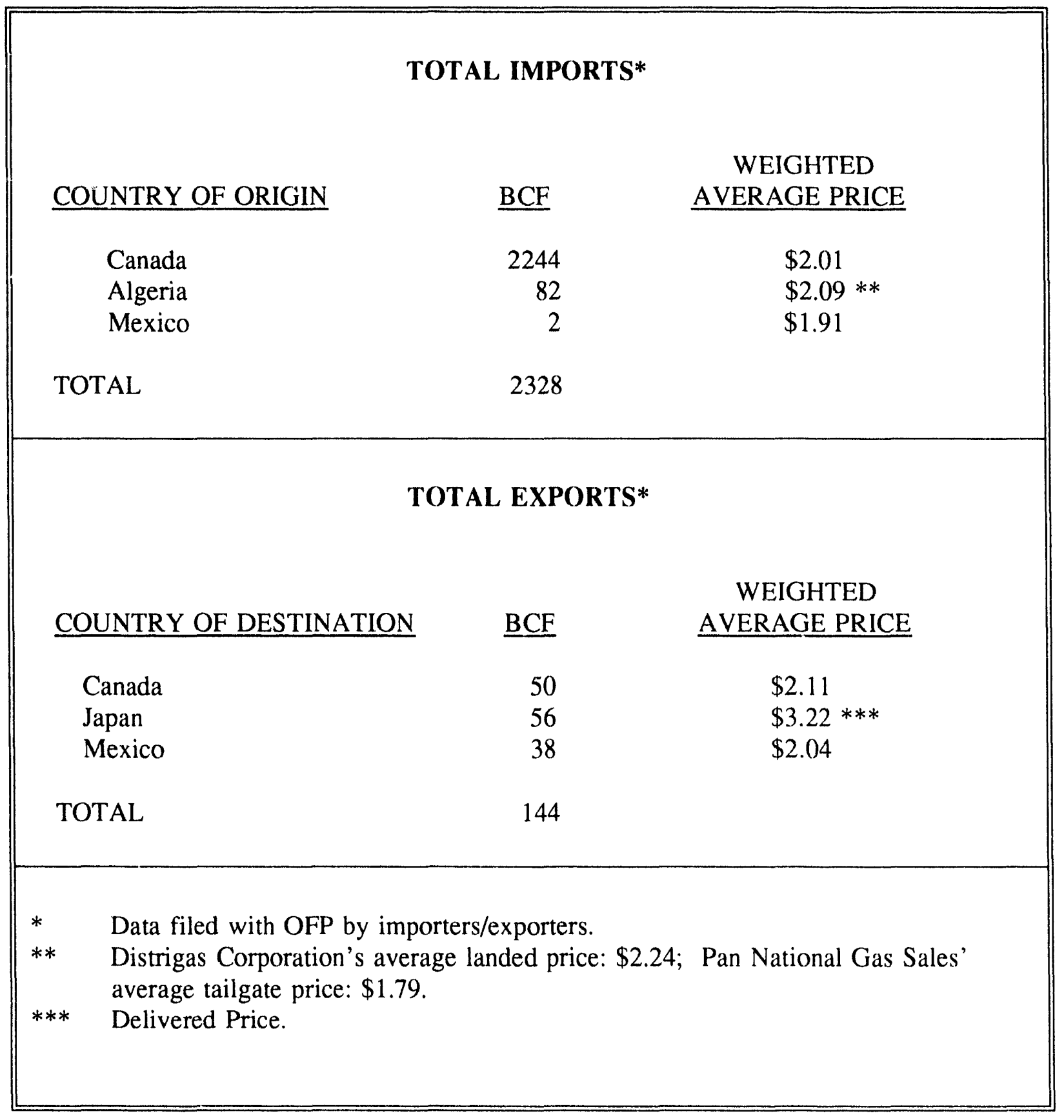




\section{NATURAL GAS IMPORTS \\ Volumes and Weighted Average Prices \\ By Point of Entry and Type of Authorization \\ $(10 / 01 / 93-12 / 31 / 93)$}

POINT OF ENTRY

VOLUME

WEIGHTED

AVERAGE

(MMCF)

PRICE

(\$MMBTU)

\section{LONG-TERM AUTHORIZATIONS}

Babb, Montana

$\begin{array}{rl}478.00 & 1.93 \\ 15.56 & 2.97 \\ 70,035.97 & 1.88 \\ 15,173.98 & 2.18^{*} \\ 1,104.46 & 3.19 \\ 2,151.58 & 2.73 \\ 1,748.37 & 2.64 \\ 4,612.26 & 1.75^{* *} \\ 4,250.64 & 2.97 \\ 47,832.64 & 2.56 \\ 2,553.86 & 2.44 \\ 63,140.34 & 2.22 \\ 76,210.81 & 1.71 \\ 21,016.63 & 1.96 \\ 57,058.40 & 2.53 \\ 135.48 & 2.63 \\ 2,001.23 & 1.47\end{array}$

Champlain, New York

2.97

Eastport, Idaho

$2.18^{*}$

Everett, Massachusetts

Grand Island, New York

Highgate Springs, Vermont

3.19

International Falls, MN

Lake Charles, Louisiana

Massena, New York

Niagara Falls, New York

North Troy, Vermont

Noyes, Minnesota

Port of Morgan, Montana

Sumas, Washington

Waddington, New York

Warroad, Minnesota

Whitlash, Montana

$369,520.22$

2.13

\section{SHORT-TERM AUTHORIZATIONS}

Babb, Montana

Detroit, Michigan

Eastport, Idaho

Grand Island, NY

Hidalgo, Texas

Highgate Spr., VT

Lake Charles, LA

Massena, New York

Niagara Falls, NY

North Tro ${ }_{j}$, Vermont

Noyes, Minnesota

Port of Morgan, Montana

St. Clair, Michigan

Sumas, Washington

Waddington, NY

$\begin{array}{ll}3,068.17 & 1.88\end{array}$

$\begin{array}{ll}1,990.31 & 2.13\end{array}$

$\begin{array}{ll}76,078.20 & 1.97\end{array}$

$21.09 \quad 2.93$

$\begin{array}{ll}1,727.73 & 1.91\end{array}$

$\begin{array}{ll}51.95 & 3.19\end{array}$

$281.02 \quad 1.80^{* *}$

$\begin{array}{ll}409.35 & 2.09\end{array}$

$\begin{array}{ll}15,357.59 & 2.22\end{array}$

$30.18 \quad 2.49$

$31,224.53 \quad 2.18$

$46,063.30 \quad 1.70$

$1,839.37 \quad 2.30$

$\begin{array}{ll}65,565.74 & 1.90\end{array}$

$\begin{array}{ll}7,321.51 & 2.28\end{array}$

$\begin{array}{ll}251,030.03 & 1.95\end{array}$

TOTAL SHORT-TERM IMPORTS

* Average landed price

** Average tailgate price

Note: Import figures in this table may vary slightly from sum of components in report due to independent rounding in calculations. 
LONG-TERM IMPORTS

ACTUAL TAKES AS A PERCENT OF AUTHORIZED VOLUMES

\section{IMPORTER/EXPORTER}

AG-ENERGY, L.P./Home Oil Company Limited

ANR PIPELINE COMPANY/ProGas Limited

BOSTON GAS COMPANY/ALberta Northeast Gas (WGM Ltd. 1)

BOSTON GAS COMPANY/ESSO Resources Canada Limited

BOUNDARY GAS, INC. /Transcanada Pipel ines Limited

BROOKLYN LINION GAS COMPANY/ALberta NE Gas (AEC Dil \& Gas company)

BROOKLYN UNION GAS COMPANY/Alberta Northeast Gas (WGM Ltd. 1)

BROOKLYN UNION GAS COMPANY/ALberta Northeast Gas (WGM Ltd. 2)

BROOKLYN UNION GAS COMPANY/Alberta Northeast Gas (ProGas)

BROOKLYN UNION GAS COMPANY/Alberta Northeast Gas (Atcor)

CASCADE NATURAL GAS CORPORATION/Mobil Oil Canada, Ltd.

CASCADE NATURAL GAS CORPORATION/Canadian Hydrocarbons Marketing

CASCADE NATURAL GAS CORPORATION/Canadian Hydrocarbons Market ing

CASCADE NATURAL GAS CORPORATION/Canadian Hydrocarbons Marketing

CENTRAL HUDSON COMPANY/ALberta Northeast Gas (WGM Ltd. 1)

CENTRAL HUDSON COMPANY/Alberta Northeast Gas (WGM Ltd.

CENTRAL HUDSON COMPANY/ALberta Northeast Gas (ProGas)

CENTRAL HUDSON COMPANY/ALberta Northeast Gas (Atcor)

CENTRAL HUDSON COMPANY/ALberta NE Gas (AEC Oil \& Gas Company)

CITY OF BURBANK, CALIFORNIA/Westcoast Gas Services

CITY OF GLENDALE, CALIFORNIA/westcoast Gas Services

CITY OF PASADENA, CALIFORNIA/Westcoast Gas Services

COLONIAL NATURAL GAS/Alberta Northeast Gas (WGM Ltd. 1)

COMMONWEALTH GAS COMPANY/ALberta Northeast Gas (WGM Ltd. 1)

CONNECTICUT NATURAL GAS/ALberta Northeast Gas (WGM Ltd. 1)

CONNECTICUT NATURAL GAS/Alberta Northeast Gas (WGM Ltd. 2)

CONNECTICUT NATURAL GAS/Alberta Northeast Gas (ProGas)

CONNECTICUT NATURAL GAS/ALberta Northeast Gas (Atcor)

CONNECTICUT NATURAL GAS/ALberta NE Gas (AEC Oil \& Gas Company)

CONSOLIDATED EDISON COMPANY OF N.Y. /ALberta NE Gas (WGM LTd. 1)

CONSOLIDATED EDISON COMPANY OF M.Y./ALLERT NE GOS (UGM LTd.

CONSOLIDATED EDISON COMPANY OF N.Y./ALberta NE Gas (ProGas)

CONSOLIDATED EDISON COMPANY OF N.Y. /ALberta NE Gas (ProGas)

CONSOLIDATED EDISON COMPANY OF N. Y. ALberta NE Gas (AEC Oil \& Gas)

CONSOLIDATED EDISON COMPANY OF NEW YORK/AmOCO Canada

CONSUMERS POWER COMPANY/WGM LTd.

CONSUMERS POWER COMPANY1/Norcen Energy Resources Ltd.

CONSUMERS POWER COMPANY2/HUSKY Oil

CONSUMERS POWER COMPANY3/Shell Canada Limited

CONSUMERS POWER COMPANY4/POCO Petroleums, Ltd.

CONSUMERS POWER COMPANY5/North Canadian Oils, Lto.

CRESTAR ENERGY MARKETING CORPORATION/Crestar Energy

\begin{tabular}{rr}
1992 & 1993 \\
4th & 1 st \\
qtr & gtr \\
\hline 0 & 0 \\
50 & 19 \\
100 & 100 \\
67 & 100 \\
100 & 100 \\
99 & 99 \\
100 & 100 \\
100 & 100 \\
100 & 100 \\
100 & 100 \\
97 & 97 \\
0 & 0 \\
0 & 0 \\
0 & 0 \\
101 & 100 \\
109 & 103 \\
102 & 101 \\
100 & 99 \\
64 & 95 \\
0 & 0 \\
0 & 0 \\
0 & 0 \\
100 & 98 \\
100 & 98 \\
100 & 100 \\
99 & 99 \\
100 & 100 \\
101 & 96 \\
102 & 100 \\
100 & 100 \\
100 & 100 \\
100 & 100 \\
100 & 100 \\
102 & 101 \\
99 & 99 \\
100 & 88 \\
100 & 88 \\
100 & 88 \\
100 & 88 \\
92 & 88 \\
91 & 86 \\
0 & 0 \\
& \\
& \\
100 &
\end{tabular}

WEIGHTED AVERAGE PRICE ( $\$$ /MMBTU) OF TAKES

$\begin{array}{rrrrr}1992 & 1993 & 1993 & 1993 & 1993 \\ \text { 4th } & 1 \text { st } & \text { 2nd } & \text { 3rd } & \text { 4th } \\ \text { gtr } & \text { qtr } & \text { qtr } & \text { qtr } & \text { qtr } \\ \text { N/A } & \text { N/A } & \text { N/A } & \text { N/A } & 2.19 \\ 2.92 & 4.21 & 2.85 & 2.32 & 2.27 \\ 2.95 & 2.91 & 2.51 & 2.37 & 2.55 \\ 3.51 & 3.04 & 3.09 & 2.61 & 2.90 \\ 2.92 & 2.89 & 2.50 & 2.35 & 2.53 \\ 2.97 & 2.91 & 2.51 & 2.37 & 2.54 \\ 3.00 & 2.91 & 2.51 & 2.37 & 2.55 \\ 2.90 & 2.91 & 2.51 & 2.37 & 2.55 \\ 2.96 & 2.91 & 2.53 & 2.37 & 2.55 \\ 2.96 & 2.91 & 2.51 & 2.37 & 2.55 \\ 2.44 & 2.68 & 2.62 & 2.31 & 2.70 \\ \text { N/A } & \text { N/A } & \text { N/A } & \text { N/A } & 2.51 \\ \text { N/A } & \text { N/A } & \text { N/A } & \text { N/A } & 2.30 \\ \text { N/A } & \text { N/A } & \text { N/A } & 1.63 & 2.24 \\ 2.97 & 2.91 & 2.51 & 2.37 & 2.55 \\ 3.02 & 2.91 & 2.51 & 2.37 & 2.55 \\ 3.02 & 2.91 & 2.53 & 2.37 & 2.55 \\ 2.96 & 2.91 & 2.51 & 2.37 & 2.55 \\ 3.11 & 2.91 & 2.83 & 2.37 & 2.54 \\ \text { N/A } & \text { N/A } & \text { N/A } & \text { N/A } & 1.74 \\ \text { N/A } & \text { N/A } & \text { N/A } & \text { N/A } & 1.69 \\ \text { N/A } & \text { N/A } & \text { N/A } & \text { N/A } & 1.74 \\ 2.95 & 2.91 & 2.51 & 2.37 & 2.55 \\ 2.95 & 2.91 & 2.51 & 2.37 & 2.55 \\ 3.00 & 2.91 & 2.51 & 2.37 & 2.55 \\ 2.98 & 2.91 & 2.51 & 2.37 & 2.55 \\ 2.98 & 2.91 & 2.53 & 2.37 & 2.55 \\ 3.00 & 2.91 & 2.82 & 2.37 & 2.55 \\ 3.04 & 2.91 & 2.51 & 2.37 & 2.54 \\ 2.94 & 2.91 & 2.84 & 2.37 & 2.55 \\ 2.94 & 2.91 & 2.51 & 2.37 & 2.55 \\ 2.94 & 2.91 & 2.53 & 2.37 & 2.55 \\ 2.94 & 2.91 & 2.51 & 2.37 & 2.55 \\ 2.94 & 2.91 & 2.51 & 2.37 & 2.54 \\ 2.61 & 2.72 & 2.64 & 2.42 & 2.51 \\ 2.00 & 2.08 & 2.03 & 1.90 & 1.68 \\ 2.07 & 2.16 & 2.12 & 1.99 & 1.77 \\ 2.02 & 2.10 & 2.06 & 1.94 & 1.72 \\ 2.00 & 2.07 & 2.04 & 1.91 & 1.69 \\ 2.12 & 2.11 & 2.00 & 1.88 & 1.66 \\ 2.03 & 2.04 & 1.98 & 1.87 & 1.66 \\ \text { N/A } & \text { N/A } & \text { N/A } & \text { N/A } & 2.32 \\ & & & & \end{array}$


LONG-TERM IMPORTS

\section{ACTUAL TAKES AS A PERCENT OF AUTHORIZED VOLUMES}

\section{IMPORTER/EXPORTER}

DARTMOUTH POWER ASSOCIATES, L.P. / Canadian Natural Gas Resources DARTMOUTH POWER ASSOCIATES, L.P. /Anderson Oil \& Gas inc. DARTMOUTH POWER ASSOCIATES, L.P. /Excel Energy Inc DARTMOUTH POWER ASSOCIATES, L.P./Remington Energy Ltd. DISTRIGAS CORPORATION (88-37-LNG)/Sonatrach

DISTRIGAS CORPORATION (89-16-LNG)/Sonatrach

DOME PETROLEUM CORPORATION/DOme Petroleum Limited

ELIZABETHTOWN GAS COMPANY/WGM Ltd.

ENCOGEN FOUR PARTNERS, L.P./Sceptre Resources Limited

ENCOGEN NORTHWEST, L.P./CanWest Gas Supply, Inc.

ENERGYNORTH, INC./Alberta Northeast Gas (WGM Ltd. 1)

ESSEX COUNTY GAS CO./Alberta Northeast Gas (WGM Ltd. 1)

FULTON COGENERATION ASSOCIATES/Star Oil \& Gas Limited

FULTON COGENERATION ASSOCIATES/OMV (Canada) Limited

GRANITE STATE GAS TRANSMISSION: INC. / Shell Canada Limited

GRANITE STATE GAS TRANSMISSION, INC./Shell Canada Limited

GRANITE STATE GAS TRANSMISSION, INC. /Direct Energy Marketing Ltd. GREAT FALLS GAS COMPANY/Shell Canada Limited

IGI RESOURCES, INC./MObil Oil Canada

INDECK ENERGY SERVICES OF OSWEGO, INC. / Indeck Gas Supply corp.

INDECK-YERKES ENERGY SERVICES, INC. / Indeck Gas Supply corp.

INDECK-YERKES LIMITED PARTNERSHIP/Northstar Energy Corporation

KAMINE/BESICORP CARTHAGE L.P./Renaissance Energy Ltd.

KAMINE/BESICORP NATURAL DAM L.P. /North Canadian Marketing Inc.

KAMINE/BESICORP SOUTH GLENS FALLS L.P. /Renaissance Energy Ltd.

KAMINE/BESICORP SYRACUSE L.P. /North Canadian Marketing Inc.

KCS ENERGY MARKETING, INC./Ramarro Resources Ltd.

LOCKPORT ENERGY ASSOCIATES, L.P./ProGas Limited

LONG ISLAND LIGHTING COMPANY/Alberta Northeast Gas (WGM Ltd. 1)

LONG ISLAND LIGHTING COMPANY/Alberta Northeast Gas (WGM Ltd. 2)

LONG ISLAND LIGHTING COMPANY/ALberta Northeast Gas (ProGas)

ONG ISLAND IGHTING COMPANY/Alberta Northeast Gas (Atcor)

LONG ISLAND LIGHTING COMPANY/ALberta NE Gas (AEC Oil \& Gas)

MEGAN-RACINE ASSOCIATES, INC. /WGM Ltd.

MICHIGAN CONSOLIDATED GAS COMPANY/WGM Ltd.

MIDLAND COGENERATION VENTURE1/Norcen Energy Resources Ltd.

MIDLAND COGENERATION VENTURE2/Shell Canada Limited

MIDLAND COGENERATION VENTURE3/Canterra Energy Limited

MIDLAND COGENERATION VENTURE4/TransCanada Pipelines Limited

MIDLAND COGENERATION VENTURE5/POCO Petroleums, Ltd.

MIDLAND COGENERATION VENTURE6/North Canadian oils, Ltd.

MINNEGASCO INC. /TransCanada Pipelines Limited

N Y STATE ELECTRIC \& GAS CO./Alberta Northeast Gas (WGM Ltd. 2)

$\begin{array}{rrrrr}1992 & 1993 & 1993 & 1993 & 1993 \\ \text { 4th } & 1 \text { st } & \text { 2nd } & \text { 3rd } & \text { 4th } \\ \text { qtr } & \text { qtr } & \text { qtr } & \text { qtr } & \text { qtr } \\ 53 & 66 & 52 & 71 & 63 \\ 55 & 50 & 49 & 82 & 72 \\ 62 & 51 & 40 & 83 & 65 \\ 59 & 53 & 44 & 77 & 60 \\ \text { N/A } & \text { N/A } & \text { N/A } & \text { N/A } & \text { N/A } \\ \text { V/A } & \text { N/A } & \text { N/A } & \text { N/A } & \text { N/A } \\ 100 & 99 & 88 & 97 & 33 \\ 67 & 100 & 100 & 99 & 100 \\ 0 & 0 & 0 & 30 & 72 \\ 0 & 0 & 0 & 82 & 85 \\ 99 & 78 & 98 & 98 & 99 \\ 99 & 98 & 98 & 98 & 99 \\ 87 & 79 & 83 & 92 & 98 \\ 30 & 0 & 0 & 0 & 0 \\ 93 & 100 & 63 & 52 & 88 \\ 65 & 98 & 82 & 83 & 96 \\ 0 & 0 & 0 & 0 & 96 \\ 66 & 101 & 101 & 61 & 104 \\ 83 & 100 & 100 & 100 & 100 \\ 77 & 63 & 78 & 79 & 78 \\ 66 & 75 & 76 & 64 & 73 \\ 0 & 37 & 109 & 109 & 110 \\ 84 & 79 & 57 & 81 & 80 \\ 0 & 0 & 0 & 0 & 58 \\ 93 & 85 & 66 & 86 & 72 \\ 0 & 0 & 0 & 0 & 8 \\ 95 & 98 & 50 & 97 & 100 \\ 43 & 97 & 86 & 74 & 79 \\ 100 & 100 & 100 & 100 & 100 \\ 100 & 100 & 100 & 100 & 100 \\ 100 & 100 & 100 & 100 & 100 \\ 99 & 99 & 99 & 99 & 99 \\ 99 & 99 & 99 & 99 & 99 \\ 76 & 79 & 81 & 72 & 86 \\ 90 & 90 & 91 & 87 & 90 \\ 100 & 100 & 100 & 100 & 100 \\ 99 & 99 & 100 & 100 & 100 \\ 100 & 97 & 100 & 98 & 99 \\ 100 & 100 & 100 & 100 & 100 \\ 100 & 100 & 100 & 100 & 100 \\ 67 & 51 & 100 & 90 & 91 \\ 87 & 99 & 49 & 39 & 89 \\ 99 & 99 & 99 & 99 & 99\end{array}$

\section{WEIGHTED AVERAGE PRICE ( $\$$ /MMBTU) OF TAKES}

$\begin{array}{rrrrr}1992 & 1993 & 1993 & 1993 & 1993 \\ \text { 4th } & 1 \text { st } & \text { 2nd } & \text { 3rd } & \text { 4th } \\ \text { qtr } & \text { qtr } & \text { qtr } & \text { qtr } & \text { qtr } \\ 2.83 & 2.70 & 2.67 & 2.57 & 2.42 \\ 2.83 & 2.68 & 2.67 & 2.57 & 2.43 \\ 2.81 & 2.68 & 2.67 & 2.58 & 2.43 \\ 2.81 & 2.68 & 2.67 & 2.57 & 2.43 \\ 2.81 & 2.84 & 1.73 & 2.11 & 2.21 \\ \text { N/A } & 2.17 & 2.13 & 2.13 & 2.11 \\ 2.06 & 1.98 & 2.37 & 2.17 & 1.54 \\ 2.42 & 2.55 & 2.97 & 2.60 & 2.97 \\ \text { N/A } & \text { N/A } & \text { N/A } & 2.61 & 2.86 \\ \text { N/A } & \text { N/A } & \text { N/A } & 1.84 & 1.84 \\ 2.95 & 2.91 & 2.51 & 2.37 & 2.55 \\ 2.95 & 2.91 & 2.51 & 2.37 & 2.55 \\ 2.34 & 2.56 & 2.47 & 2.43 & 2.49 \\ 2.02 & \text { N/A } & \text { N/A } & \text { N/A } & \text { N/A } \\ 2.89 & 2.72 & 3.28 & 3.49 & 2.42 \\ 2.85 & 2.65 & 2.95 & 2.65 & 2.50 \\ \text { N/A } & \text { N/A } & \text { N/A } & \text { N/A } & 2.51 \\ 1.51 & 1.53 & 1.57 & 1.86 & 1.93 \\ 1.78 & 1.78 & 1.78 & 1.76 & 1.78 \\ 2.71 & 2.52 & 2.47 & 2.52 & 2.52 \\ 2.41 & 2.22 & 2.19 & 2.28 & 2.25 \\ \text { N/A } & 1.79 & 1.78 & 1.73 & 2.06 \\ 2.49 & 2.81 & 2.97 & 2.48 & 2.63 \\ \text { N/A } & \text { N/A } & \text { N/A } & \text { N/A } & 2.51 \\ 2.08 & 2.17 & 2.07 & 1.94 & 2.09 \\ \text { N/A } & \text { N/A } & \text { N/A } & \text { N/A } & 4.65 \\ 2.45 & 2.66 & 3.10 & 2.63 & 2.56 \\ 4.02 & 3.01 & 3.11 & 3.27 & 3.28 \\ 2.98 & 2.91 & 2.51 & 2.37 & 2.55 \\ 2.98 & 2.91 & 2.51 & 2.37 & 2.55 \\ 2.98 & 2.91 & 2.53 & 2.37 & 2.55 \\ 2.97 & 2.91 & 2.51 & 2.37 & 2.55 \\ 2.95 & 2.91 & 2.51 & 2.37 & 2.54 \\ 3.19 & 3.09 & 2.81 & 3.01 & 2.84 \\ 2.62 & 1.97 & 2.51 & 2.33 & 2.35 \\ 1.91 & 1.83 & 1.87 & 1.86 & 1.84 \\ 1.73 & 1.75 & 1.78 & 1.78 & 1.76 \\ 1.90 & 1.91 & 1.93 & 1.93 & 1.91 \\ 1.73 & 1.75 & 1.77 & 1.77 & 1.74 \\ 1.74 & 1.90 & 2.03 & 1.91 & 1.77 \\ 2.45 & 2.68 & 2.35 & 2.40 & 2.38 \\ 2.66 & 2.28 & 2.71 & 2.79 & 2.58 \\ 3.03 & 2.91 & 2.51 & 2.37 & 2.55\end{array}$


IONG-TERM IMPORTS

ACTUAL TAKES AS A PERCENT OF AUTHORIZED VOLUMES

IMPORTER/EXPORTER

N.Y. STATE ELECTRIC \& GAS CO./Alberta Northeast Gas (ProGas) N.Y. STATE ELECTRIC \& GAS CO./Alberta Northeast Gas (Atcor) N.Y STATE ELECTRIC \& GAS Co./Alberta NE Gas (AEC Oil \& Gas Co.) N.Y. STATE ELECTRIC \& GAS CO./Progas Limited N.Y. STATE ELECTRIC \& GAS CO./Progas Limited N.Y. STATE ELECTRIC \& GASUTION COPPORATION/Alberta NE Gas (WGM Ltd) NATTONAL FUEL GAS DISTRIBUTION CORPORATIONALLETTa NE Ga NATURAL GAS PIPELIE COMPANY OF AMERICA/WGM Ltd.

NATURAL GAS PIPELINE COMPANY OF AMERICA WGM Ltd.

NEW ENGLAND POWER COMPANY/Talisman Energy Ino.

NEW ENGLAND POWER COMPANY/Renaissance Energy Limited

NEW ENGLAND POWER COMPANY/Sceptre Resources Limited

NEW ENGLAND POWER COMPANY/Transwest Energy Inc.

NEW JERSEY NATURAL GAS/ALberta Northeast Gas (WGM Ltd. 1)

NEW JERSEY NATURAL GAS/Alberta Northeast Gas (WGM Ltd. 2)

NEW JERSEY NATURAL GAS/ALberta Nor theast Gas (ProGas;

NEW JERSEY NATURAL GAS/Aiberta Northeast Gas (Atcor)

NEW JERSEY NATURAL GAS/Alberta NE Gas (AEC Oil \& Gas Company)

NIAGARA MOHAWK POWER CORPORATION/WGM Ltd.

NORTH JERSEY ENERGY ASSOCIATES/ProGas Limited

NORTHEAST ENERGY ASSOCIATES/ProGas Limited

NORTHERN MINNESOTA UTILITIES/WGM LTd.

NORTHERN MINNESOTA UTILITIES - EASTERN MARKET/WGM Ltd.

NORTHERN MINNESOTA UTILITIES - WESTERN MARKET/WGM Ltd.

NORTHERN MINNESOTA UTILITIES/CITY OF WARROAD/WGM Ltd.

NORTHERN NATURAL GAS COMPANY/WEst coast Gas Services

NORTHERN NATURAL GAS COMPANY/WGM Ltd.

NORTHERN NATURAL GAS COMPANY/Mobil Gas Canada

NORTHERN STATES POWER COMPANY/Canadian Occidental Petroleum Company NORTHERN STATES POWER COMPANY/Amoco Canada Petroleum Company Ltd.

NORTHERN STATES POWER COMPANY/ProGas Limited

NORTHWEST NATURAL GAS COMPANY/CanWest Gas Supply

NORTHUEST NATURAL GAS COMPANY/Amoco Canada Petroleum Co. Ltd.

NORTHWEST NATURAL GAS COMPANY/POCO Petroleums Limited

NORTHWEST NATURAL GAS COMPANY/SUmmit Resources Limited

NORTHWEST NATURAL GAS COMPANY/Westcoast Gas Services

NORTHWEST NATURAL GAS COMPANY/Canadian Hydrocarbons Marketing

NORTHWEST PIPELINE CORPORATION/Canadian Hydrocarbons Marketing

NORTHWEST PIPELINE CORPORATION/Canadian Hydrocarbons Marketing

NW ALASKAN PIPELINE CO-Northern Natural/Pan-Alberta Gas Ltd.

NW ALASKAN PIPELINE CO-Pacific Interstate/ an-Alberta Gas Ltd.

NW ALASKAN PIPELINE CO-Pan-Alberta Gas US/Pan-Alberta Gas Ltd.

OCEAN STATE POWER/ProGas Limited

OCEAN STATE POWER II/ProGas Limited

$\begin{array}{rrrrr}1992 & 1993 & 1993 & 1993 & 1993 \\ \text { 4th } & 1 \text { st } & 2 \text { nd } & 3 \text { rd } & 4 \text { th } \\ \text { qtr } & \text { qtr } & \text { qtr } & \text { qtr } & \text { qtr } \\ \text { 100 } & 101 & 101 & 101 & 101 \\ 99 & 98 & 97 & 99 & 99 \\ 101 & 102 & 102 & 102 & 102 \\ 0 & 0 & 0 & 0 & 20 \\ 0 & 0 & 0 & 0 & 1 \\ 100 & 100 & 99 & 100 & 100 \\ 58 & 95 & 60 & 97 & 82 \\ 66 & 77 & 64 & 94 & 98 \\ 0 & 0 & 0 & 0 & 39 \\ 0 & 0 & 0 & 0 & 48 \\ 0 & 0 & 0 & 0 & 39 \\ 0 & 0 & 0 & 0 & 37 \\ 100 & 100 & 100 & 100 & 100 \\ 99 & 99 & 99 & 99 & 99 \\ 100 & 100 & 100 & 100 & 100 \\ 100 & 99 & 99 & 101 & 99 \\ 101 & 102 & 102 & 102 & 102 \\ 12 & 95 & 100 & 100 & 100 \\ 95 & 97 & 98 & 90 & 91 \\ 99 & 100 & 100 & 100 & 101 \\ 85 & 84 & 53 & 85 & 83 \\ 67 & 69 & 54 & 48 & 64 \\ 83 & 114 & 35 & 25 & 72 \\ 27 & 33 & 10 & 6 & 26 \\ 85 & 80 & 47 & 56 & 16 \\ 79 & 99 & 86 & 76 & 29 \\ 73 & 98 & 55 & 51 & 17 \\ 65 & 100 & 100 & 98 & 100 \\ 64 & 104 & 104 & 102 & 104 \\ 65 & 99 & 90 & 98 & 98 \\ 88 & 94 & 99 & 100 & 97 \\ 81 & 96 & 100 & 97 & 101 \\ 0 & 0 & 0 & 0 & 65 \\ 0 & 0 & 0 & 0 & 62 \\ 0 & 0 & 0 & 0 & 55 \\ 0 & 0 & 0 & 0 & 14 \\ 39 & 55 & 41 & 39 & 11 \\ 84 & 77 & 84 & 97 & 91 \\ 97 & 100 & 97 & 87 & 98 \\ 99 & 101 & 82 & 92 & 90 \\ 33 & 0 & 0 & 0 & 0 \\ 99 & 100 & 100 & 101 & 100 \\ 100 & 100 & 100 & 101 & 100\end{array}$

WEIGHTED AVERAGE PRICE ( $\$$ /MMBTU) OF TAKES

$\begin{array}{rrrrr}\text { 1992 } & 1993 & 1993 & 1993 & 1993 \\ \text { 4th } & 1 \text { st } & \text { 2nd } & \text { 3rd } & \text { 4th } \\ \text { qtr } & \text { qtr } & \text { qtr } & \text { qtr } & \text { qtr } \\ \text { 3.03 } & 2.91 & 2.53 & 2.37 & 2.55 \\ 3.04 & 2.91 & 2.51 & 2.37 & 2.55 \\ 3.06 & 2.91 & 2.51 & 2.37 & 2.54 \\ \text { N/A } & \text { N/A } & \text { N/A } & \text { N/A } & 2.39 \\ \text { N/A } & \text { N/A } & \text { N/A } & \text { N/A } & 3.33 \\ 2.84 & 2.90 & 2.50 & 2.36 & 2.53 \\ 2.29 & 1.71 & 2.16 & 1.88 & 1.98 \\ 2.95 & 2.23 & 2.73 & 2.42 & 2.39 \\ \text { N/A } & \text { N/A } & \text { N/A } & \text { N/A } & 2.59 \\ \text { N/A } & \text { N/A } & \text { N/A } & \text { N/A } & 2.78 \\ \text { N/A } & \text { N/A } & \text { N/A } & \text { N/A } & 2.43 \\ \text { N/A } & \text { N/A } & \text { N/A } & \text { N/A } & 3.07 \\ 2.92 & 2.91 & 2.51 & 2.37 & 2.55 \\ 2.92 & 2.91 & 2.51 & 2.37 & 2.55 \\ 2.92 & 2.91 & 2.53 & 2.37 & 2.55 \\ 2.91 & 2.91 & 2.51 & 2.37 & 2.55 \\ 2.92 & 2.91 & 2.51 & 2.37 & 2.54 \\ 2.40 & 2.29 & 2.26 & 2.07 & 2.20 \\ 2.16 & 2.33 & 2.32 & 2.36 & 2.35 \\ 2.82 & 2.95 & 2.95 & 2.94 & 2.93 \\ 2.12 & 2.13 & 2.74 & 2.40 & 2.39 \\ 2.22 & 2.35 & 2.53 & 2.61 & 2.64 \\ 2.26 & 2.09 & 3.04 & 3.57 & 2.50 \\ 2.86 & 3.10 & 6.34 & 6.86 & 3.00 \\ 2.25 & 1.89 & 2.21 & 2.03 & 1.97 \\ 2.43 & 2.02 & 2.43 & 2.34 & 2.19 \\ 2.09 & 1.65 & 2.15 & 1.97 & 1.91 \\ 2.21 & 2.24 & 1.95 & 1.93 & 2.24 \\ 2.14 & 1.98 & 1.97 & 1.96 & 2.37 \\ 2.28 & 2.33 & 2.00 & 1.97 & 2.20 \\ 1.94 & 1.91 & 1.68 & 1.79 & 1.83 \\ 1.87 & 1.82 & 1.61 & 1.61 & 1.76 \\ \text { N/A } & \text { N/A } & \text { N/A } & \text { N/A } & 2.02 \\ \text { N/A } & \text { N/A } & \text { N/A } & \text { N/A } & 2.23 \\ \text { N/A } & \text { N/A } & \text { N/A } & \text { N/A } & 2.14 \\ \text { N/A } & \text { N/A } & \text { N/A } & \text { N/A } & 3.31 \\ 1.85 & 1.73 & 1.80 & 1.83 & 1.83 \\ 2.33 & 1.94 & 2.16 & 1.82 & 1.97 \\ 2.05 & 1.96 & 1.98 & 1.97 & 1.97 \\ 1.81 & 1.46 & 1.61 & 1.58 & 1.58 \\ 1.63 & \text { N/A } & \text { N/A } & \text { N/A } & \text { N/A } \\ 1.93 & 1.97 & 2.02 & 2.06 & 2.05 \\ 1.93 & 1.97 & 2.01 & 2.06 & 2.05 \\ & & & & \end{array}$




\section{ACTUAL TAKES AS A PERCENT OF AUTHORIZED VOLUMES}

\section{IMPORTER/EXPORTER}

OCEAN STATE POWER II/WGM Ltd.

ORCHARD GAS CORPORATION/PrOGas Limited

PACIFIC GAS TRANSMISSION COMPANY/ALberta \& Southern Gas Company

PAN NATIONAL GAS SALES, INC./Sonatrading

PAWTUCKET POWER ASSOCIATES/Tarragon Oil \& Gas Limited

PAUTUCKET POWER ASSOCIATES/HOme Oil Company Limited

PEOPLES NATURAL GAS COMPANY/HGM Ltd.

PEPPEREL POWER ASSOCIATES, L.P. /WGM Ltd.

POWER CITY PARTNERS, L.P. / Husky Oil Operations Ltd.

PROGAS U.S.A. INC./Progas Limited

PROJECT ORANGE ASSOCIATES, L.P./NOranda, Inc.

PUBLIC SERVICE ELECTRIC \& GAS/Alberta Northeast Gas (WGM Ltd. 1) ROCHESTER GAS AND ELECTRIC CORPORATION/Westcoast Gas Services SALMON RESOURCES LTD./Shell Canada Limited

SALMON RESOURCES LTD. -ENRON GAS MARKETING/Shell Canada Limited SALMON RESOURCES LTD. -MIDWEST GAS/Shell Canada Limited

SAN DIEGO GAS \& ELECTRIC COMPANY/BOW Valley Industries Ltd.

SAN DIEGO GAS \& ELECTRIC COMPANY/Canadian Hunter Marketing Ltd.

SAN DIEGO GAS \& ELECTRIC COMPANY/Husky Oil Operations Ltd.

SAN DIEGO GAS \& ELECTRIC COMPANY/SUmmit Resources Ltd.

SARANAC POWER PARTNERS, L.P. /Shell Canada Limited

SELKIRK COGEN PARTNERS, L.P. / Paramount Resources Ltd.

SOUTHEASTERM MICHIGAN GAS COMPANY/WGM Ltd.

SOUTHERN CALIFORNIA EDISON COMPANY/Shell Canada Limited

SOUTHERN CALIFORNIA EDISON COMPANY/AEC Oil \& Gas Company

SOUTHERN CALIFORNIA EDISON COMPANY/Imperial Oil Resources Limited SOUTHERN CALIFORNIA EDISON COMPANY/WGM LTd.

SOUTHERN CONNECTICUT GAS/Alberta Northeast Gas (WGM Ltd. 1) SOUTHERN CONNECTICUT GAS/Alberta Northeast Gas (WGM Ltd. 2) SOUTHERN CONNECTICUT GAS/ALberta Northeast Gas (ProGas)

SOUTHERN CONNECTICUT GAS/ALberta Northeast Gas (Atcor)

SOUTHERN CONNECTICUT GAS/ALberta NE Gas (AEC Oil \& Gas Company)

ST. LAURENCE GAS COMPANY INC. /Niagara Gas Transmission Ltd.

SUMAS COGENERATION COMPANY, L.P. /ENCO GaS, Ltd.

SUMAS COGENERATION COMPANY, L.P. / Canadian Hydrocarbons Marketing TENNESSEE GAS PIPELINE COMPANY/ProGas Limited

TENNESSEE GAS PIPELINE COMPANY/KannGaz Producers Ltd.

TENNESSEE GAS PIPELINE COMPANY/TransCanada Pipelines Limited

TEXAS EASTERN TRANSMISSION CORPORATION/PrOGas Limited

TEXAS EASTERN TRANSMISSION CORPORATION/ProGas Limited

THE MONTANA POWER COMPANY/Canadian-Montana Pipeline company

TM STAR FUEL COMPANY/Canwest Gas Supply

TRANSCO ENERGY MARKETING COMPANY/Canstates Gas Marketing

$\begin{array}{rrrrr}1992 & 1993 & 1993 & 1993 & 1993 \\ \text { 4th } & 1 \text { st } & \text { 2nd } & \text { 3rd } & \text { 4th } \\ \text { qtr } & \text { qtr } & \text { qtr } & \text { gtr } & \text { qtr } \\ 100 & 100 & 100 & 102 & 100 \\ 67 & 100 & 100 & 100 & 89 \\ 83 & 96 & 85 & 79 & 27 \\ \text { N/A } & \text { N/A } & \text { N/A } & \text { N/A } & \text { N/A } \\ 57 & 93 & 25 & 97 & 91 \\ 53 & 67 & 82 & 92 & 87 \\ 0 & 87 & 31 & 13 & 60 \\ 58 & 73 & 72 & 79 & 101 \\ 32 & 65 & 61 & 63 & 84 \\ 0 & 0 & 0 & 0 & 100 \\ \text { N/A } & \text { N/A } & \text { N/A } & \text { N/A } & \text { N/A } \\ 100 & 100 & 100 & 100 & 100 \\ 0 & 0 & 0 & 0 & 66 \\ 93 & 100 & 102 & 89 & 101 \\ 66 & 98 & 98 & 92 & 99 \\ 66 & 96 & 83 & 86 & 93 \\ 0 & 0 & 0 & 0 & 61 \\ 0 & 0 & 0 & 0 & 67 \\ 0 & 0 & 0 & 0 & 66 \\ 0 & 0 & 0 & 0 & 33 \\ 0 & 0 & 0 & 0 & 0 \\ 68 & 90 & 85 & 74 & 92 \\ 100 & 100 & 96 & 67 & 100 \\ 0 & 0 & 0 & 0 & 66 \\ 0 & 0 & 0 & 0 & 45 \\ 0 & 0 & 0 & 0 & 32 \\ 0 & 0 & 0 & 0 & 44 \\ 99 & 100 & 100 & 100 & 100 \\ 99 & 99 & 100 & 100 & 100 \\ 100 & 100 & 100 & 100 & 100 \\ 100 & 98 & 101 & 101 & 101 \\ 100 & 99 & 99 & 96 & 99 \\ 49 & 52 & 27 & 17 & 43 \\ 0 & 0 & 77 & 76 & 97 \\ 0 & 0 & 90 & 90 & 81 \\ 13 & 40 & 17 & 12 & 0 \\ 18 & 14 & 21 & 19 & 25 \\ 65 & 67 & 64 & 67 & 68 \\ 30 & 7 & 0 & 0 & 0 \\ 26 & 4 & 0 & 0 & 0 \\ 48 & 51 & 25 & 27 & 43 \\ 0 & 0 & 0 & 0 & 59 \\ 74 & 100 & 88 & 80 & 100 \\ & & & & \end{array}$

WEIGHTED AVERAGE PRICE ( $5 / M M B T U)$ OF TAKES

\begin{tabular}{|c|c|c|c|c|}
\hline $\begin{array}{r}1992 \\
\text { 4th }\end{array}$ & $\begin{array}{r}1993 \\
\text { 1st }\end{array}$ & $\begin{array}{r}1993 \\
\text { 2nd }\end{array}$ & $\begin{array}{r}1993 \\
3 \text { rd }\end{array}$ & $\begin{array}{r}1993 \\
\text { 4th }\end{array}$ \\
\hline gtr & qtr & qtr & qtr & gtr \\
\hline $\begin{array}{l}1.94 \\
2.63\end{array}$ & $\begin{array}{l}1.97 \\
2.63\end{array}$ & $\begin{array}{l}2.02 \\
2.15\end{array}$ & $\begin{array}{l}2.06 \\
2.72\end{array}$ & $\begin{array}{l}2.05 \\
2.60\end{array}$ \\
\hline 2.01 & 2.01 & 1.99 & 2.19 & 1.90 \\
\hline 1.78 & $N / A$ & 1.82 & 1.75 & 1.75 \\
\hline 2.81 & 2.54 & 2.66 & 2.19 & 2.33 \\
\hline 2.80 & 2.49 & 2.77 & 2.23 & 2.35 \\
\hline N/A & 2.10 & 3.11 & 3.66 & 3.35 \\
\hline 1.70 & 1.70 & 1.70 & 1.70 & 1.70 \\
\hline 3.82 & 3.35 & 3.05 & 2.86 & 3.20 \\
\hline $\mathrm{N} / \mathrm{A}$ & N/A & N/A & $N / A$ & 2.06 \\
\hline 2.31 & 2.33 & 2.50 & 2.43 & 2.48 \\
\hline 2.94 & 2.91 & 2.51 & 2.37 & 2.55 \\
\hline$N / A$ & N/A & N/A & N/A & 2.99 \\
\hline 2.87 & 3.00 & 2.93 & 2.91 & 2.88 \\
\hline 1.63 & 1.32 & 1.40 & 1.46 & 1.71 \\
\hline 1.33 & 1.49 & 1.66 & 1.51 & 1.66 \\
\hline $\mathrm{N} / \mathrm{A}$ & N/A & $N / A$ & N/A & 1.22 \\
\hline N/A & N/A & N/A & N/A & 1.26 \\
\hline$N / A$ & N/A & N/A & N/A & 1.24 \\
\hline$N / A$ & N/A & N/A & N/A & 1.02 \\
\hline N/A & $N / A$ & N/A & $N / A$ & 2.97 \\
\hline 2.66 & 2.52 & 2.58 & 2.45 & 2.35 \\
\hline 2.45 & 2.19 & 2.59 & 2.70 & 2.86 \\
\hline $\mathrm{N} / \mathrm{A}$ & N/A & N/A & $\mathrm{N} / \mathrm{A}$ & 1.48 \\
\hline $\mathrm{N} / \mathrm{A}$ & N/A & $N / A$ & N/A & 1.39 \\
\hline N/A & N/A & N/A & N/A & 1.39 \\
\hline$N / A$ & $N / A$ & $N / A$ & N/A & 1.39 \\
\hline 3.00 & 2.91 & 2.51 & 2.37 & 2.55 \\
\hline 2.96 & 2.91 & 2.51 & 2.37 & 2.55 \\
\hline 2.96 & 2.91 & 2.53 & 2.37 & 2.55 \\
\hline 2.96 & 2.91 & 2.51 & 2.37 & 2.55 \\
\hline 2.95 & 2.91 & 2.51 & 2.37 & 2.54 \\
\hline 2.75 & 2.16 & 3.35 & 2.22 & 2.81 \\
\hline N/A & $N / A$ & 2.02 & 2.02 & 2.05 \\
\hline $\mathrm{N} / \mathrm{A}$ & $N / A$ & 1.63 & 1.63 & 1.57 \\
\hline 4.12 & 2.71 & 4.65 & 4.39 & N/A \\
\hline 5.13 & 4.39 & 5.57 & 4.39 & 4.55 \\
\hline 2.97 & 2.35 & 2.83 & 2.64 & 2.69 \\
\hline 3.40 & 3.18 & N/A & N/A & N/A \\
\hline 4.69 & 6.90 & 2.65 & N/A & $N / A$ \\
\hline 1.54 & 1.55 & 1.54 & 1.49 & 1.47 \\
\hline N/A & N/A & N/A & N/A & 1.83 \\
\hline 2.91 & 2.65 & 2.76 & 2.54 & 2.65 \\
\hline
\end{tabular}


LONG-TERM IMPORTS

\section{ACTUAL TAKES AS A PERCENT OF AUTHORIZED VOLUMES}

IMPORTER/EXPORTER

TRANSCO ENERGY MARKETING COMPANY-BG\&E/Canstates Gas Marketing TRANSCO ENERGY MARKETING COMPANY-LILCO/Canstates Gas Marketing TRANSCO ENERGY MARKETING COMPANY-PSE\&/Canstates Gas Marketing VALLEY GAS COMPANY/Alberta Northeast Gas (WGM Ltd, 1)

VERMONT GAS SYSTEMS INC. / WGM Ltd.

VIKING GAS TRANSMISSION COMPANY/TransCanada Pipelines Limited WASHINGTON NATURAL GAS COMPANY/AmOCO Canada

WASHINGTON NATURAL GAS COMPANY/MObil Oil Canada

WASHINGTON NATURAL GAS COMPANY/POCO Petroleums Ltd.

UASHINGTON NATURAL GAS COMPANY/Canadian Hydrocarbons Marketing

HASHINGTON MATURAL GAS COMPANY/Canadian Hydrocarbons Marketing

WASHIMGTON HATER POUER COMPANY/Canadian Hydrocarbons Marketing

WASHINGTON WATER POWER COMPANY/AEC Oil \& Gas Company

WASHINGTON WATER POUER COMPANY/AEC OIL \& Gas COMPTY

WASHINGTON WATER POWER COMPANY/Amerada Hess Canada Ltd.

WESTERN GAS MARKETING INC. /WGM Ltd.

WESTERN GAS MARKETING INC. / WGM Ltd.

WISCONSIN FUEL \& LIGHT COMPANY/PrOgas LI

WISCONSIN FUEL \& LIGHT COMPANY/WGM Ltd.

WISCONSIN NATURAL GAS COMPANY/PrOgas L

WISCONSIN POWER \& LIGHT COMPANY/WGM LTd.

WISCONSIN POWER \& LIGHT COMPANY/ProGas Limited

WISCONSIN POWER \& LIGHT COMPANY/WGM LTd.

WISCONSIN PUBLIC SERVICE CORPORATION/TransCanada Pipelines Limited

WISCONSIN PUBLIC SERVICE CORPORATION/PrOGas Limited

WISCONSIN PUBLIC SERVICE CORPORATION/WGM Ltd.

YANKEE GAS SERVICES CO./ALberta Northeast Gas (WGM Ltd. 1)

YANKEE GAS SERVICES CO./Alberta Northeast Gas (HGM Ltd 2)

YANKEE GAS SERVICES CO./Alberta Northeast Gas (ProGas)

YANKEE GAS SERVICES CO./ALberta Northeast Gas (Atcor)

YANKEE GAS SERVICES CO./ALberta NE Gas (AEC Oil \& Gas company)

$\begin{array}{rrrrr}1992 & 1993 & 1993 & 1993 & 1993 \\ 4 \text { th } & 1 \text { st } & 2 \text { nd } & 3 \text { rd } & 4 \text { th } \\ \text { qtr } & \text { qtr } & \text { qtr } & \text { qtr } & \text { qir } \\ 95 & 32 & 0 & 36 & 51 \\ 49 & 20 & 32 & 0 & 3 \\ 84 & 40 & 51 & 31 & 23 \\ 99 & 98 & 98 & 98 & 99 \\ 74 & 93 & 45 & 32 & 73 \\ 47 & 0 & 0 & 0 & 0 \\ 100 & 98 & 101 & 97 & 101 \\ 88 & 97 & 99 & 57 & 100 \\ 100 & 98 & 67 & 81 & 100 \\ 86 & 101 & 88 & 99 & 101 \\ 0 & 0 & 0 & 0 & 28 \\ 0 & 0 & 0 & 0 & 4 \\ 0 & 0 & 0 & 0 & 40 \\ 0 & 0 & 0 & 0 & 52 \\ 0 & 0 & 0 & 0 & 35 \\ 27 & 30 & 11 & 66 & 63 \\ 0 & 0 & 0 & 0 & 65 \\ 0 & 0 & 0 & 0 & 64 \\ 0 & 0 & 0 & 0 & 58 \\ 0 & 0 & 0 & 0 & 45 \\ 98 & 99 & 97 & 98 & 98 \\ 0 & 0 & 0 & 0 & 65 \\ 0 & 0 & 0 & 0 & 65 \\ 33 & 0 & 0 & 0 & 0 \\ 0 & 0 & 0 & 0 & 67 \\ 0 & 0 & 0 & 0 & 66 \\ 100 & 100 & 100 & 100 & 100 \\ 100 & 100 & 100 & 100 & 100 \\ 100 & 100 & 100 & 100 & 100 \\ 101 & 100 & 100 & 100 & 100 \\ 101 & 101 & 101 & 101 & 101\end{array}$

WEIGHTED AVERAGE PRICE (S/MMBTU) OF TAKES

$\begin{array}{rrrrr}1992 & 1993 & 1993 & 1993 & 1993 \\ 4 \text { th } & 1 \text { st } & \text { 2nd } & 3 r d & 4 \text { th } \\ \text { qtr } & \text { qtr } & \text { qtr } & \text { qtr } & \text { qtr } \\ 2.77 & 1.84 & \text { N/A } & 1.55 & 1.70 \\ 2.64 & 1.40 & 1.77 & \text { N/A } & 1.60 \\ 2.72 & 1.28 & 1.37 & 1.49 & 1.54 \\ 2.95 & 2.91 & 2.51 & 2.37 & 2.55 \\ 2.78 & 2.54 & 3.53 & 3.95 & 2.73 \\ 2.98 & \text { N/A } & \text { N/A } & \text { N/A } & \text { N/A } \\ 1.70 & 1.76 & 1.55 & 1.53 & 1.93 \\ 2.17 & 2.37 & 2.41 & 2.12 & 2.21 \\ 1.71 & 1.83 & 1.97 & 2.22 & 2.25 \\ 1.62 & 1.75 & 1.77 & 1.69 & 2.00 \\ \text { N/A } & \text { N/A } & \text { N/A } & \text { N/A } & 2.49 \\ \text { N/A } & \text { N/A } & \text { N/A } & \text { N/A } & 2.42 \\ \text { N/A } & \text { N/A } & \text { N/A } & \text { N/A } & 2.29 \\ \text { N/A } & \text { N/A } & \text { N/A } & \text { N/A } & 2.12 \\ \text { N/A } & \text { N/A } & \text { N/A } & \text { N/A } & 1.93 \\ 4.33 & 3.45 & 5.15 & 2.72 & 2.68 \\ \text { N/A } & \text { N/A } & \text { N/A } & \text { N/A } & 2.39 \\ \text { N/A } & \text { N/A } & \text { N/A } & \text { N/A } & 2.41 \\ \text { N/A } & \text { N/A } & \text { N/A } & \text { N/A } & 2.42 \\ \text { N/A } & \text { N/A } & \text { N/A } & \text { N/A } & 2.58 \\ 2.45 & 2.08 & 2.52 & 2.26 & 2.32 \\ \text { N/A } & \text { N/A } & \text { N/A } & \text { N/A } & 2.32 \\ \text { N/A } & \text { N/A } & \text { N/A } & \text { N/A } & 2.30 \\ 2.92 & \text { N/A } & \text { N/A } & \text { N/A } & \text { N/A } \\ \text { N/A } & \text { N/A } & \text { N/A } & \text { N/A } & 2.35 \\ \text { N/A } & \text { N/A } & \text { N/A } & \text { N/A } & 2.35 \\ 3.01 & 2.91 & 2.51 & 2.37 & 2.55 \\ 2.98 & 2.91 & 2.51 & 2.37 & 2.55 \\ 2.98 & 2.91 & 2.53 & 2.37 & 2.55 \\ 2.98 & 2.91 & 2.51 & 2.37 & 2.55 \\ 2.97 & 2.91 & 2.51 & 2.37 & 2.54 \\ & & & & \end{array}$


VOLUME AND PRICE REPORT

Long Term Imports

Volumes (MMCf/d) \& Prices (S/MMBTU) of Natural

Gas Imported During the Past 12 Months 01/01/93 - 12/31/93

\begin{tabular}{|c|c|c|c|c|c|c|c|c|c|c|c|c|c|c|c|}
\hline Long-Term Importer & $\begin{array}{l}\text { Auth. } \\
\text { Vols. }\end{array}$ & $\begin{array}{l}1993 \\
\text { Jan. }\end{array}$ & $\begin{array}{l}1993 \\
\text { Feb. }\end{array}$ & $\begin{array}{l}1993 \\
\text { Mar. }\end{array}$ & $\begin{array}{l}1993 \\
\text { Apr. }\end{array}$ & $\begin{array}{l}1993 \\
\text { May }\end{array}$ & $\begin{array}{l}1993 \\
\text { June } \\
\end{array}$ & $\begin{array}{l}1993 \\
\text { July } \\
\end{array}$ & $\begin{array}{l}1993 \\
\text { Aug. }\end{array}$ & $\begin{array}{l}1993 \\
\text { Sep. }\end{array}$ & $\begin{array}{l}1993 \\
\text { Oct. }\end{array}$ & $\begin{array}{l}1993 \\
\text { Nov. }\end{array}$ & $\begin{array}{l}1993 \\
\text { Dec. }\end{array}$ & $\begin{array}{c}4093 \\
\text { TOTAL MCf } \\
\end{array}$ & $\begin{array}{c}\text { YTD } \\
\text { TOTAL MCf } \\
\end{array}$ \\
\hline $\begin{array}{l}\text { AG-ENERGY, L.P. } \\
\text { (HOME OIL COMPANY LIMITED) } \\
\text { AVG Daily Quantity } \\
\text { Total Price (Waddington, New York) } \\
\text { Commodity Component } \\
\text { Demand Component } \\
\text { Reservation Fee Component }\end{array}$ & 17.5 & $\begin{array}{r}0.0 \\
0.00 \\
0.00 \\
0.00 \\
0.00\end{array}$ & $\begin{array}{r}0.0 \\
0.00 \\
0.00 \\
0.00 \\
0.00\end{array}$ & $\begin{array}{r}0.0 \\
0.00 \\
0.00 \\
0.00 \\
0.00\end{array}$ & $\begin{array}{r}0.0 \\
0.00 \\
0.00 \\
0.00 \\
0.00\end{array}$ & $\begin{array}{r}0.0 \\
0.00 \\
0.00 \\
0.00 \\
0.00\end{array}$ & $\begin{array}{r}0.0 \\
0.00 \\
0.00 \\
0.00 \\
0.00\end{array}$ & $\begin{array}{r}0.0 \\
0.00 \\
0.00 \\
0.00 \\
0.00\end{array}$ & $\begin{array}{r}0.0 \\
0.00 \\
0.00 \\
0.00 \\
0.00\end{array}$ & $\begin{array}{r}0.0 \\
0.00 \\
0.00 \\
0.00 \\
0.00\end{array}$ & $\begin{array}{r}0.0 \\
0.00 \\
0.00 \\
0.00 \\
0.00\end{array}$ & $\begin{array}{r}5.4 \\
2.19 \\
1.36 \\
0.83 \\
0.00\end{array}$ & $\begin{array}{r}8.8 \\
2.19 \\
1.36 \\
0.83 \\
0.00\end{array}$ & 435,315 & 435,315 \\
\hline $\begin{array}{l}\text { ANR PIPELINE COMPANY (1) } \\
\text { (PROGAS LIMITED) } \\
\text { AVG Daily Quantity } \\
\text { Total Price (Noyes, Minnesota) } \\
\text { Commodity Component } \\
\text { Demand Component } \\
\text { Reservation Fee Component }\end{array}$ & 75.0 & $\begin{array}{l}32.0 \\
2.83 \\
1.79 \\
1.04 \\
0.00\end{array}$ & $\begin{array}{r}5.7 \\
8.37 \\
1.43 \\
6.94 \\
0.00\end{array}$ & $\begin{array}{r}5.6 \\
7.95 \\
1.69 \\
6.26 \\
0.00\end{array}$ & $\begin{array}{r}5.3 \\
8.74 \\
1.94 \\
6.80 \\
0.00\end{array}$ & $\begin{array}{l}68.2 \\
2.95 \\
2.44 \\
0.51 \\
0.00\end{array}$ & $\begin{array}{l}74.9 \\
2.34 \\
1.85 \\
0.49 \\
0.00\end{array}$ & $\begin{array}{l}75.0 \\
2.18 \\
1.70 \\
0.48 \\
0.00\end{array}$ & $\begin{array}{l}75.0 \\
2.27 \\
1.81 \\
0.46 \\
0.00\end{array}$ & $\begin{array}{l}75.0 \\
2.52 \\
2.06 \\
0.46 \\
0.00\end{array}$ & $\begin{array}{r}74.9 \\
2.25 \\
1.79 \\
0.46 \\
0.00\end{array}$ & $\begin{array}{r}9.4 \\
2.24 \\
1.72 \\
0.52 \\
0.00\end{array}$ & $\begin{array}{l}10.4 \\
2.46 \\
1.99 \\
0.47 \\
0.00\end{array}$ & $2,924,981$ & $15,677,094$ \\
\hline $\begin{array}{l}\text { BOSTON GAS COMPANY } \\
\text { (ALBERTA NORTHEAST GAS (WGM LTD. 1)) } \\
\text { AVG Daily Quantity } \\
\text { Total Price (Waddington, New York) } \\
\text { Commodity Component } \\
\text { Demand Component } \\
\text { Reservation Fee Component }\end{array}$ & 8.6 & $\begin{array}{r}8.6 \\
2.91 \\
1.98 \\
0.93 \\
0.00\end{array}$ & $\begin{array}{r}8.6 \\
2.91 \\
1.96 \\
0.95 \\
0.00\end{array}$ & $\begin{array}{r}8.6 \\
2.91 \\
1.95 \\
0.96 \\
0.00\end{array}$ & $\begin{array}{r}8.6 \\
2.47 \\
1.49 \\
0.98 \\
0.00\end{array}$ & $\begin{array}{r}8.6 \\
2.70 \\
1.73 \\
0.97 \\
0.00\end{array}$ & $\begin{array}{r}8.6 \\
2.37 \\
1.39 \\
0.98 \\
0.00\end{array}$ & $\begin{array}{r}8.6 \\
2.37 \\
1.39 \\
0.98 \\
0.00\end{array}$ & $\begin{array}{r}8.6 \\
2.37 \\
1.40 \\
0.97 \\
0.00\end{array}$ & $\begin{array}{r}8.6 \\
2.37 \\
1.43 \\
0.94 \\
0.00\end{array}$ & $\begin{array}{r}8.6 \\
2.29 \\
1.35 \\
0.94 \\
0.00\end{array}$ & $\begin{array}{r}8.6 \\
2.66 \\
1.73 \\
0.93 \\
0.00\end{array}$ & $\begin{array}{r}8.6 \\
2.69 \\
1.77 \\
0.92 \\
0.00\end{array}$ & 791,200 & $3,146,053$ \\
\hline $\begin{array}{l}\text { BOSTON GAS COMPANY } \\
\text { (ESSO RESOURCES CANADA LIMITED) } \\
\text { AVG Daily Quantity } \\
\text { Total Price (Waddington, New York) } \\
\text { Commodity Component } \\
\text { Demand Component } \\
\text { Reservation Fee Component }\end{array}$ & 35.0 & $\begin{array}{l}35.0 \\
3.19 \\
2.29 \\
0.90 \\
0.00\end{array}$ & $\begin{array}{r}35.0 \\
3.00 \\
2.00 \\
1.00 \\
0.00\end{array}$ & $\begin{array}{l}34.7 \\
2.94 \\
2.02 \\
0.92 \\
0.00\end{array}$ & $\begin{array}{l}35.0 \\
3.04 \\
2.10 \\
0.94 \\
0.00\end{array}$ & $\begin{array}{l}35.0 \\
3.42 \\
2.52 \\
0.90 \\
0.00\end{array}$ & $\begin{array}{r}35.0 \\
2.80 \\
1.88 \\
0.92 \\
0.00\end{array}$ & $\begin{array}{r}38.0 \\
2.46 \\
1.63 \\
0.83 \\
0.00\end{array}$ & $\begin{array}{l}38.0 \\
2.56 \\
1.74 \\
0.82 \\
0.00\end{array}$ & $\begin{array}{l}37.8 \\
2.80 \\
1.97 \\
0.83 \\
0.00\end{array}$ & $\begin{array}{l}35.0 \\
2.56 \\
1.69 \\
0.87 \\
0.00\end{array}$ & $\begin{array}{l}35.0 \\
2.99 \\
2.07 \\
0.92 \\
0.00\end{array}$ & $\begin{array}{l}35.0 \\
3.16 \\
2.28 \\
0.88 \\
0.00\end{array}$ & $3,220,083$ & $13,068,583$ \\
\hline $\begin{array}{l}\text { BOUNDARY GAS, INC. } \\
\text { (TRANSCANADA PIPELINES LIMITED) } \\
\text { AVG Daily Quant ity } \\
\text { Total Price (Niagara Falls, New York) } \\
\text { Commodity Component } \\
\text { Demand Component } \\
\text { Reservation Fee Component }\end{array}$ & 92.5 & $\begin{array}{l}92.5 \\
2.89 \\
1.98 \\
0.91 \\
0.00\end{array}$ & $\begin{array}{l}92.4 \\
2.89 \\
1.96 \\
0.93 \\
0.00\end{array}$ & $\begin{array}{r}92.3 \\
2.89 \\
1.98 \\
0.91 \\
0.00\end{array}$ & $\begin{array}{r}92.2 \\
2.45 \\
1.49 \\
0.96 \\
0.00\end{array}$ & $\begin{array}{r}92.3 \\
2.69 \\
1.73 \\
0.96 \\
0.00\end{array}$ & $\begin{array}{l}92.3 \\
2.35 \\
1.39 \\
0.96 \\
0.00\end{array}$ & $\begin{array}{l}32.2 \\
2.35 \\
1.40 \\
0.95 \\
0.00\end{array}$ & $\begin{array}{l}95.0 \\
2.35 \\
1.39 \\
0.96 \\
0.00\end{array}$ & $\begin{array}{r}92.4 \\
2.35 \\
1.43 \\
0.92 \\
0.00\end{array}$ & $\begin{array}{l}92.5 \\
2.28 \\
1.35 \\
0.93 \\
0.00\end{array}$ & $\begin{array}{l}92.5 \\
2.65 \\
1.73 \\
0.92 \\
0.00\end{array}$ & $\begin{array}{r}92.5 \\
2.67 \\
1.77 \\
0.90 \\
0.00\end{array}$ & $8,508,988$ & $33,889,576$ \\
\hline
\end{tabular}

(1) Beginning in November 1993, the daily contract demand was reduced to 10.4 MMcf. 


\section{VOLUME AND PRICE REPORT}

Long Term Imports

Volumes (MMCf/d) \& Prices ( $\$ / M M B T 0$ ) of Natural

Gas Imported During the Past 12 Months 01/01/93- 12/31/93

\section{Long-Term Importer}

BROOKLYN UNION GAS COMPANY

(ALBERTA NE GAS (AEC OIL \& GAS COMPANY)) AVG Daily Quantity

Total Price (Waddington, New York)

Commodity Component

Demand Component

Reservation Fee Component

BROOKLYN UNION GAS COMPANY

(ALBERTA NORTHEAST GAS (WGM LTD. 1)) AVG Daily Quantity

Total Price (Waddington, New York) Commodity Component

Demand Component

Reservation Fee Component

BROOKLYN UNION GAS COMPANY

(ALBERTA NORTHEAST GAS (WGM LTD. 2))

AVG Daily Quantity

Total Price (Waddington, New York)

Commodity Component

Demand Component

Reservation Fee Component

BROOKLYN UNION GAS COMPANY

(ALBERTA NORTHEAST GAS (PROGAS))

AVG Daily Quantity

Total Price (Waddingtor, New York)

Commodity Component

Demand Component

Reservation Fee Component

BROOKLYN UNION GAS COMPANY

(ALBERTA NORTHEAST GAS (ATCOR))

AVG Daily Quantity

Total Price (Waddington, New York)

Commodity Component

Demand Component

Reservation Fee Component

8.2

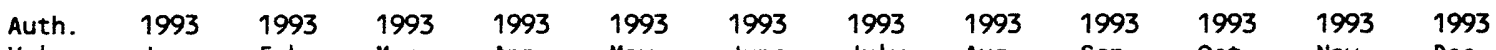

Vols. Jan. Feb. Mar. Apr. May June July Aug. Sep. Oct. Nov. Dec.

4.0

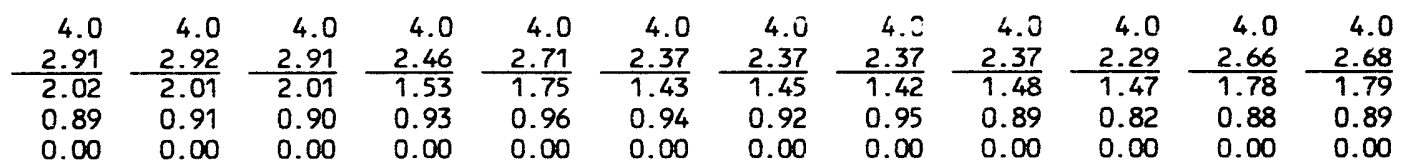

24.

\begin{tabular}{lllllllllllll}
24.0 & 24.0 & 24.0 & 24.0 & 24.0 & 24.0 & 24.0 & 24.0 & 24.0 & 24.0 & 24.0 & 24.0 \\
2.91 & 2.91 & 2.91 & 2.47 & $\frac{2.70}{1.93}$ & $\frac{2.37}{1.39}$ & $\frac{2.37}{1.39}$ & $\frac{2.37}{1.40}$ & $\frac{2.37}{1.43}$ & $\frac{2.29}{1.35}$ & $\frac{2.66}{1.73}$ & $\frac{2.69}{1.77}$ \\
\hline 1.98 & 1.96 & 1.95 & 1.49 & 1.73 & 1.39 & 0.98 & 0.97 & 0.94 & 0.94 & 0.93 & 0.92 \\
0.93 & 0.95 & 0.96 & 0.98 & 0.97 & 0.98 & 0.98 & 0.00 & 0.00 & 0.00 & 0.00 & 0.00 & 0.00
\end{tabular}

\begin{tabular}{lrrr}
18.0 & 18.0 & 18.0 & 18.0 \\
2.91 & 2.91 & $\frac{2.91}{2.95}$ & $\frac{2.47}{1.49}$ \\
\hline 1.98 & 1.96 & 1.95 & \\
0.93 & 0.95 & 0.96 & 0.98 \\
0.00 & 0.00 & 0.00 & 0.00
\end{tabular}

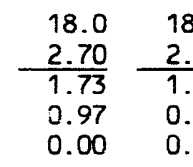

$18.0 \quad 18.0$

\begin{tabular}{llllll}
18.0 & 18.0 & 18.0 & 18.0 & 18.0 \\
& 2.37 & 2.37 & 2.29 & 2.66 & $\frac{2.69}{1.37}$ \\
\hline 1.40 & 1.43 & 1.35 & 1.73 & 1.77 \\
0.97 & 0.94 & 0.94 & 0.93 & 0.92 \\
0.00 & 0.00 & 0.00 & 0.00 & 0.00
\end{tabular}

15.8
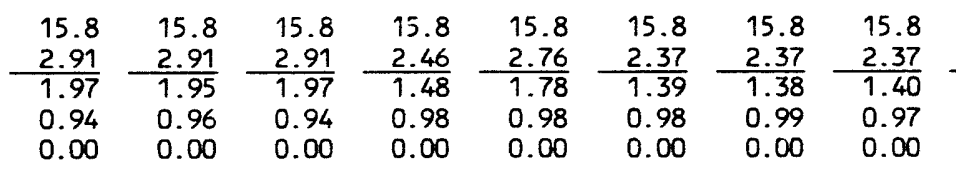

$\frac{15.8}{2.36}$

$\begin{array}{lll}15.8 & 15.8 & 15.8\end{array}$ $1.42 \quad \frac{2.30}{1.35}-\frac{2.67}{1.73} \frac{2.69}{1.73}$

$0.00 \quad 0.00$

$0.00 \quad 0.00$

0.00
$0.94 \quad 0.96$

$1,457,097$

$5,797,137$

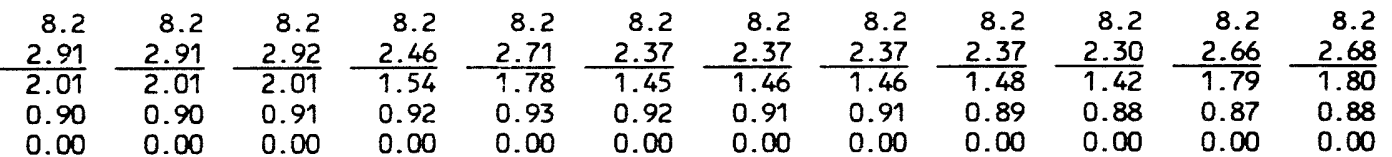

4093

TOTAL MCf TOTAL MCf

$363,585 \quad 1,446,981$

$2,208,451$

$8,784,568$

$1,656,000$

$6,588,000$

0.00

$$
0
$$$$
0.00
$$ 


\section{VOLUNE AND PRICE REPORT}

Long Term Imports

Volumes (MMCf/d) \& Prices (\$/MMBTU) of Natural

Gas Imported During the Past 12 Months $01 / 01 / 93-12 / 31 / 93$

\section{Long-Term Importer}

CASCADE NATURAL GAS CORPORATION (MOBIL OIL CANADA, LTD.)

AVG Daily Quantity

Cotal Price (Sumas, Washington) Commodity Componen Demand Component

Peservation Fee Component

CASCADE NATURAL GAS CORPORATION (CANADIAN HYDROCARBONS MARKETING)

AVG Daily Quantity

Total Price (Eastport Idaho) Commodity Component

Demand Component

Reservation Fee Component

CASCAUE NATURAL GAS CORPORATION

(CANADIAN HYDROCARBONS MARKETING)

AVG Daily Quantity

Cotal Price (Sumas, Washington)

Commodity Componen

Demand Component

Reservation Fee Component

CASCADE NATURAL GAS CORPORATION

(CANADIAN HYDROCARBONS MARKETING)

AVG Daily Quantity

Total Price (Sumas, Washington)

Commodity Component

Demand Component

Reservation Fee Component

CENTRAL HUDSON COMPANY

(ALBERTA NORTHEAST GAS (WGM LTD. 1))

AVG Daily Quant ity

Total Price (Waddington, New York)

Commodity Component

Demand Component

Reservation Fee Component

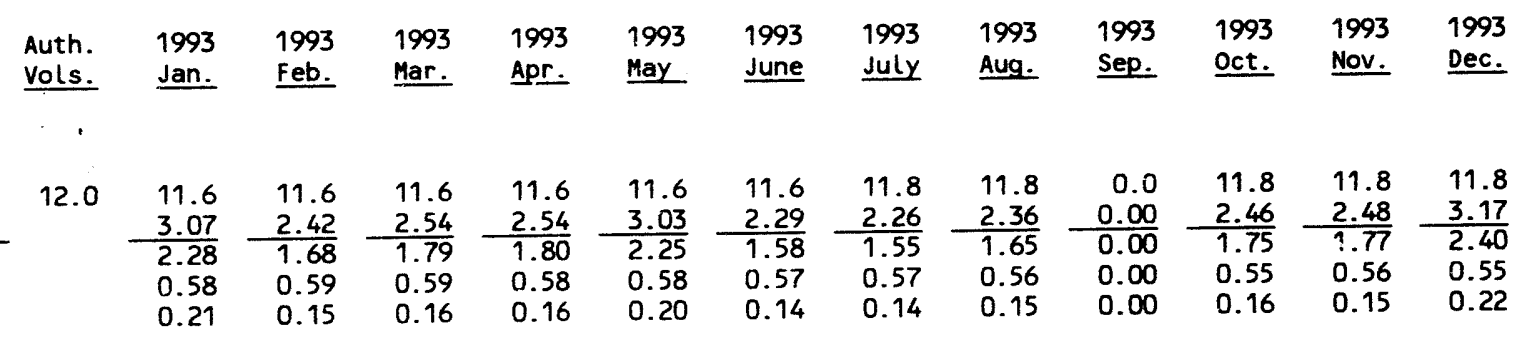

33.

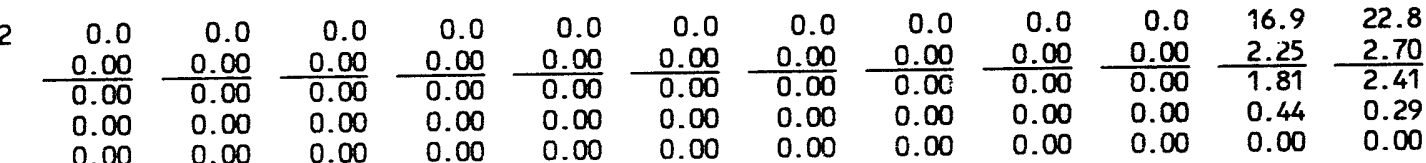

10.0

\begin{tabular}{lrr}
0.0 & 0.0 & 0.0 \\
0.00 & 0.00 & 0.00 \\
\hline 0.00 & 0.00 & 0.00 \\
0.00 & 0.00 & 0.00 \\
0.00 & 0.00 & 0.00
\end{tabular}

\begin{tabular}{l}
0.0 \\
0.00 \\
\hline 0.00 \\
0.00 \\
0.00
\end{tabular}

\begin{tabular}{r}
0.0 \\
0.00 \\
\hline 0.00 \\
0.00
\end{tabular}

\begin{tabular}{l}
0.0 \\
0.00 \\
\hline 0.00
\end{tabular}

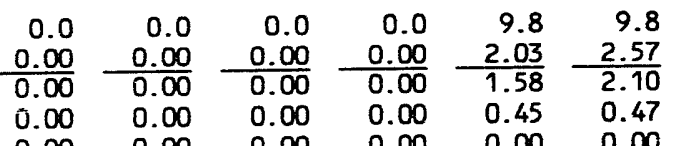

0.00

0.00

$0.00 \quad 0.00$

0.00

$0.00 \quad 0.00$

4.9

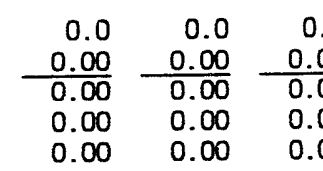

\begin{tabular}{ll}
0 & 0.0 \\
0 & 0.00 \\
\hline 00 & 0.00 \\
00 & 0.00 \\
0.00
\end{tabular}

\begin{tabular}{rrrr}
0.0 & 0.0 & 4.9 & 4.9 \\
0.00 & 0.00 & 1.62 & 1.62 \\
\hline & 0.00 & 1.12 & 1.12
\end{tabular}

\begin{tabular}{rrrrr}
.9 & 4.9 & 4.9 & 4.9 & 4.9 \\
62 & 1.64 & 2.24 & 2.24 & 2.24 \\
\cline { 3 - 5 } & 1.14 & 1.74 & 1.74 & 1.74 \\
20 & 0.20 & 0.20 & 0.20 & 0.20 \\
30 & 0.30 & 0.30 & 0.30 & 0.30
\end{tabular}

451,426

902,851

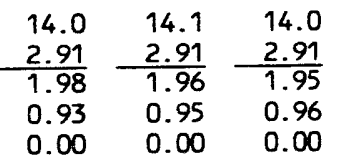

\begin{tabular}{r}
14.1 \\
2.47 \\
\hline 1.49 \\
0.98 \\
0.00
\end{tabular}

$14.1 \quad 14.1$

\begin{tabular}{l}
14.1 \\
2.37 \\
\hline
\end{tabular}

$14.1 \quad 14.1$

\begin{tabular}{ll}
14.1 & 14.1 \\
2.37 & 2 \\
\hline
\end{tabular}

$4.1 \quad 14.1$

$14.1 \quad 14.1$

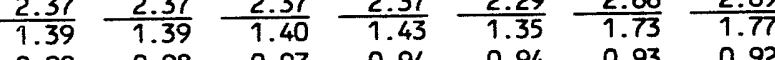

$\begin{array}{llllllllll}0.00 & 0.00 & 0.00 & 0.00 & 0.00 & 0.00 & 0.00 & 0.00 & 0.00 & 0.00\end{array}$
4093 TOTAL MCf TOTAL MCF

$1,083,420 \quad 3,924,042$

$1,215,003 \quad 1,215,003$

598,623

598,623 


\section{VOLUME AND PRICE REPORT \\ Long Term Imports}

Volumes (MMCf/d) \& Prices (S/MMBTU) of Natural

Gas Imported During the Past 12 Months $01 / 01 / 93^{-} 12 / 31 / 93$

\section{Long-Term Importer}

CENTRAL HUDSON COMPANY

(ALBERTA NORTHEAST GAS (WGM LTD. 2))

AVG Daily Quantity

Total Price (Waddington, New York

Commodity Component

Reservation Fee Component

CENTRAL HUDSON COMPANY

(ALBERTA NORTHEAST GAS (PROGAS))

AVG Daily Quantity

Total Price (Waddington, New York)

Commodity Component

Demand Component

Reservation Fee Component

CENTRAL HUDSON COMPANY

(ALBERTA NORTHEAST GAS (ATCOR))

AVG Daily Quantity

Total Price (Waddington, New York)

Commodity Component

Demand Component

Reservation Fee Component

CENTRAL HUDSON COMPANY

(ALBERTA NE GAS (AEC OIL \& GAS COMPANY))

AVG Daily Quantity

Total Price (Waddington, New York)

Commodity Component

Demand Component

Reservation Fee Component

CITY OF BURBANK, CALIFORNIA

(WESTCOAST GAS SERVICES)

AVG Daily Quantity

Total Price (Eastport, Idaho)

Commodity Component

Reservation, Fee Component

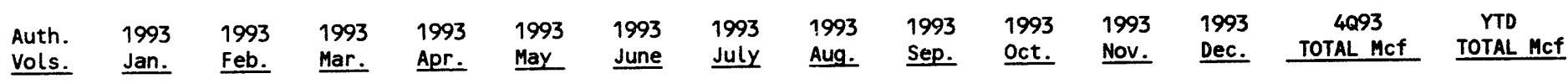

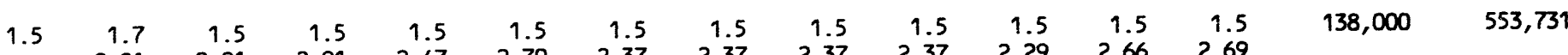

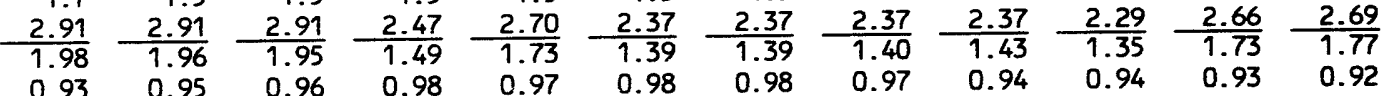

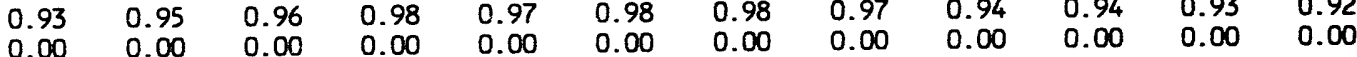

$121,440 \quad 482,357$

1.3 \begin{tabular}{rrrrrrrrrrrrr}
1.3 & 1.3 & 1.3 & 1.3 & 1.3 & 1.3 & 1.3 & 1.3 & 1.3 & 1.3 & 1.3 & 1.3 \\
& 2.91 & 2.91 & $\frac{2.91}{1.97}$ & $\frac{2.46}{1.48}$ & $\frac{2.76}{1.78}$ & $\frac{2.37}{1.39}$ & $\frac{2.37}{1.38}$ & $\frac{2.37}{1.40}$ & $\frac{2.36}{1.42}$ & $\frac{2.30}{1.35}$ & $\frac{2.67}{1.73}$ & $\frac{2.69}{1.73}$ \\
\hline 1.97 & 1.95 & 1.97 & 0.96 &
\end{tabular} $\begin{array}{llllllllllll}0.94 & 0.96 & 0.94 & 0.98 & 0.98 & 0.98 & 0.99 & 0.97 & 0.94 & 0.95 & 0.94 & 0.96\end{array}$

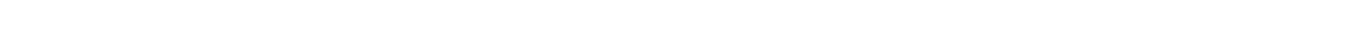

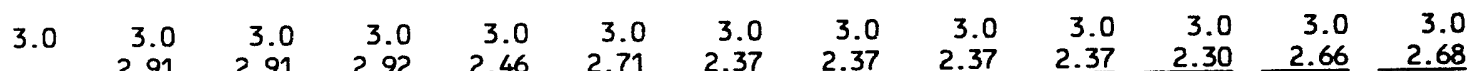
\begin{tabular}{llllllllllll}
2.91 & $\frac{2.91}{2.01}$ & $\frac{2.92}{2.01}$ & $\frac{2.46}{1.54}$ & $\frac{2.71}{1.78}$ & $\frac{2.37}{1.45}$ & $\frac{2.37}{1.46}$ & $\frac{2.37}{1.46}$ & $\frac{2.37}{1.48}$ & $\frac{2.30}{1.42}$ & $\frac{2.66}{1.79}$ & $\frac{2.68}{1.80}$ \\
\hline & 0.90 & 0.91 & 0.92 & 0.93 & 0.92 & 0.91 & 0.91 & 0.89 & 0.88 & 0.87 & 0.88
\end{tabular}

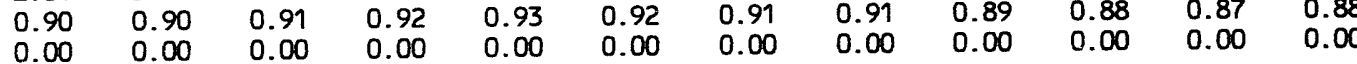

$274,528 \quad 1,090,675$

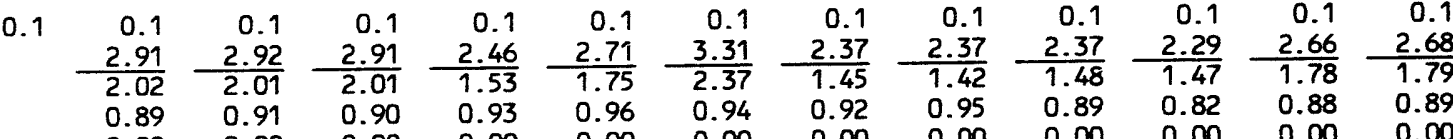

$8,832 \quad 35,055$ 
VOLULE AND PRICE REPORT

Long Term Imports

Volumes (MMcf/d) \& Prices (S/MMBTU) of Natural

Gas Imported During the Past 12 Months $01 / 01 / 93-12 / 31 / 93$

\section{Long-Term Importer}

CITY OF GLENDALE, CALIFORNIA (WESTCOAST GAS SERVICES)

AVG Daily Quantity

Total Price (Eastport, Idaho)

Commodity Component

Demand Component

Reservation Fee Component

CITY OF PASADENA, CALIFORNIA

(WESTCOAST GAS SERVICES)

AVG Daily Quantity

Total Price (Eastport, Idaho)

Commodity Component

Demand Component

Reservation Fee Component

COLONIAL NATURAL GAS

(ALBERTA NORTHEAST GAS (WGM LTD. 1))

AVG Daily Quantity

Total Price (Waddington, New York)

Commodity Component

Demand Component

Reservation Fee Component

COMMONWEALTH GAS COMPANY

(ALBERTA NORTHEAST GAS (WGM LTD. 1))

AVG Daily Quantity

Total Price (Waddington, New York)

Commodity Component

Demand Component

Reservation Fee Component

CONNECTICUT NATURAL GAS

(ALBERTA NORTHEAST GAS (WGM LTD. 1))

AVG Daily Quantity

Total Price (Waddington, New York) Commodity Component

Demand Component

\begin{tabular}{|c|c|c|c|c|c|c|c|c|c|c|c|c|c|c|}
\hline $\begin{array}{l}\text { Auth. } \\
\text { Vols. }\end{array}$ & $\begin{array}{l}1993 \\
\text { Jan. } \\
\end{array}$ & $\begin{array}{l}1993 \\
\text { Feb. }\end{array}$ & $\begin{array}{l}1993 \\
\text { Mar. }\end{array}$ & $\begin{array}{l}1993 \\
\text { Apr. }\end{array}$ & $\begin{array}{l}1993 \\
\text { May }\end{array}$ & $\begin{array}{l}1993 \\
\text { June }\end{array}$ & $\begin{array}{l}1993 \\
\text { July } \\
\end{array}$ & $\begin{array}{l}1993 \\
\text { Aug. }\end{array}$ & $\begin{array}{l}1993 \\
\text { Sep. }\end{array}$ & $\begin{array}{l}1993 \\
\text { oct. }\end{array}$ & $\begin{array}{l}1993 \\
\text { Nov. } \\
\end{array}$ & $\begin{array}{l}1993 \\
\text { Dec. }\end{array}$ & $\begin{array}{c}4093 \\
\text { TOTAL Mcf } \\
\end{array}$ & $\begin{array}{c}\text { YTD } \\
\text { TOTAL MCf } \\
\end{array}$ \\
\hline 4.1 & $\begin{array}{r}0.0 \\
0.00 \\
0.00 \\
0.00 \\
0.00\end{array}$ & $\begin{array}{r}0.0 \\
0.00 \\
0.00 \\
0.00 \\
0.00\end{array}$ & $\begin{array}{r}0.0 \\
0.00 \\
0.00 \\
0.00 \\
0.00\end{array}$ & $\begin{array}{r}0.0 \\
0.00 \\
0.00 \\
0.00 \\
0.00\end{array}$ & $\begin{array}{r}0.0 \\
0.00 \\
0.00 \\
0.00 \\
0.00\end{array}$ & $\begin{array}{r}0.0 \\
0.00 \\
0.00 \\
0.00 \\
0.00\end{array}$ & $\begin{array}{r}0.0 \\
0.00 \\
0.00 \\
0.00 \\
0.00\end{array}$ & $\begin{array}{r}0.0 \\
0.00 \\
0.00 \\
0.00 \\
0.00\end{array}$ & $\begin{array}{r}0.0 \\
0.00 \\
0.00 \\
0.00 \\
0.00\end{array}$ & $\begin{array}{r}0.0 \\
0.00 \\
0.00 \\
0.00 \\
0.00\end{array}$ & $\begin{array}{r}4.0 \\
1.71 \\
1.44 \\
0.27 \\
0.00\end{array}$ & $\begin{array}{r}4.0 \\
1.68 \\
1.44 \\
0.24 \\
0.00\end{array}$ & 246,001 & 246,001 \\
\hline 4.1 & $\begin{array}{r}0.0 \\
0.00 \\
0.00 \\
0.00 \\
0.00\end{array}$ & $\begin{array}{r}0.0 \\
0.00 \\
0.00 \\
0.00 \\
0.00\end{array}$ & $\begin{array}{r}0.0 \\
0.00 \\
0.00 \\
0.00 \\
0.00\end{array}$ & $\begin{array}{r}0.0 \\
0.00 \\
0.00 \\
0.00 \\
0.00\end{array}$ & $\begin{array}{r}0.0 \\
0.00 \\
0.00 \\
0.00 \\
0.00\end{array}$ & $\begin{array}{r}0.0 \\
0.00 \\
0.00 \\
0.00 \\
0.00\end{array}$ & $\begin{array}{r}0.0 \\
0.00 \\
0.00 \\
0.00 \\
0.00\end{array}$ & $\begin{array}{r}0.0 \\
0.00 \\
0.00 \\
0.00 \\
0.00\end{array}$ & $\begin{array}{r}0.0 \\
0.00 \\
0.00 \\
0.00 \\
0.00\end{array}$ & $\begin{array}{r}0.0 \\
0.00 \\
0.00 \\
0.00 \\
0.00\end{array}$ & $\begin{array}{r}4.3 \\
1.74 \\
1.44 \\
0.30 \\
0.00\end{array}$ & $\begin{array}{r}4.3 \\
1.74 \\
1.44 \\
0.30 \\
0.00\end{array}$ & 263,044 & 263,044 \\
\hline 6.0 & $\begin{array}{r}5.9 \\
2.91 \\
1.98 \\
0.93 \\
0.00\end{array}$ & $\begin{array}{r}5.9 \\
2.91 \\
1.96 \\
0.95 \\
0.00\end{array}$ & $\begin{array}{r}5.9 \\
2.91 \\
1.95 \\
0.96 \\
0.00\end{array}$ & $\begin{array}{r}5.9 \\
2.47 \\
1.49 \\
0.98 \\
0.00\end{array}$ & $\begin{array}{r}5.9 \\
2.70 \\
1.73 \\
0.97 \\
0.00\end{array}$ & $\begin{array}{r}5.9 \\
2.37 \\
1.39 \\
0.98 \\
0.00\end{array}$ & $\begin{array}{r}5.9 \\
2.37 \\
1.39 \\
0.98 \\
0.00\end{array}$ & $\begin{array}{r}5.9 \\
2.37 \\
1.40 \\
0.97 \\
0.00\end{array}$ & $\begin{array}{r}5.9 \\
2.37 \\
1.43 \\
0.94 \\
0.00\end{array}$ & $\begin{array}{r}5.9 \\
2.29 \\
1.35 \\
0.94 \\
0.00\end{array}$ & $\begin{array}{r}5.9 \\
2.66 \\
1.73 \\
0.93 \\
0.00\end{array}$ & $\begin{array}{r}6.0 \\
2.69 \\
1.77 \\
0.92 \\
0.00\end{array}$ & 548,040 & $2,165,059$ \\
\hline 4.5 & $\begin{array}{r}4.5 \\
2.91 \\
1.98 \\
0.93 \\
0.00\end{array}$ & $\begin{array}{r}4.4 \\
2.91 \\
1.96 \\
0.95 \\
0.00\end{array}$ & $\begin{array}{r}4.4 \\
2.91 \\
1.95 \\
0.96 \\
0.00\end{array}$ & $\begin{array}{r}4.4 \\
2.47 \\
1.49 \\
0.98 \\
0.00\end{array}$ & $\begin{array}{r}4.4 \\
2.70 \\
1.73 \\
0.97 \\
0.00\end{array}$ & $\begin{array}{r}4.4 \\
2.37 \\
1.39 \\
0.98 \\
0.00\end{array}$ & $\begin{array}{r}4.4 \\
2.37 \\
1.39 \\
0.98 \\
0.00\end{array}$ & $\begin{array}{r}4.4 \\
2.37 \\
1.40 \\
0.97 \\
0.00\end{array}$ & $\begin{array}{r}4.4 \\
2.37 \\
1.43 \\
0.94 \\
0.00\end{array}$ & $\begin{array}{r}4.5 \\
2.29 \\
1.35 \\
0.94 \\
0.00\end{array}$ & $\begin{array}{r}4.5 \\
2.66 \\
1.73 \\
0.93 \\
0.00\end{array}$ & $\begin{array}{r}4.5 \\
2.69 \\
1.77 \\
0.92 \\
0.00\end{array}$ & 413,923 & $1,627,166$ \\
\hline 11.5 & $\begin{array}{l}11.6 \\
2.91 \\
1.98 \\
0.93 \\
0.00\end{array}$ & $\begin{array}{l}11.5 \\
2.91 \\
1.96 \\
0.95 \\
0.00\end{array}$ & $\begin{array}{l}11.5 \\
2.91 \\
1.95 \\
0.96 \\
0.00\end{array}$ & $\begin{array}{l}11.5 \\
2.47 \\
1.49 \\
0.98 \\
0.00\end{array}$ & $\begin{array}{l}11.5 \\
2.70 \\
1.73 \\
0.97 \\
0.00\end{array}$ & $\begin{array}{l}11.5 \\
2.37 \\
1.39 \\
0.98 \\
0.00\end{array}$ & $\begin{array}{l}11.5 \\
2.37 \\
1.39 \\
0.98 \\
0.00\end{array}$ & $\begin{array}{l}11.5 \\
2.37 \\
1.40 \\
0.97 \\
0.00\end{array}$ & $\begin{array}{l}11.5 \\
2.37 \\
1.43 \\
0.94 \\
0.00\end{array}$ & $\begin{array}{l}11.5 \\
2.29 \\
1.35 \\
0.94 \\
0.00\end{array}$ & $\begin{array}{l}11.5 \\
2.66 \\
1.73 \\
0.93 \\
0.00\end{array}$ & $\begin{array}{l}11.5 \\
2.69 \\
1.77 \\
0.92 \\
0.00\end{array}$ & $1,057,966$ & $4,210,758$ \\
\hline
\end{tabular}




\section{VOLUNE AND PRICE REPORT}

Volumes (MM Long Term Imports

Volumes (MMCf/d) \& Prices (\$/MMBTU) of Natural
Gas Imported During the Past 12 Months $01 / 01 / 93-12 / 31 / 93$

\section{Long-Term Importer}

CONNECTICUT NATURAL GAS (ALBERTA NORTHEAST GAS (WGM LTD. 2)) AVG Daily Quantity

Total Price (Waddington, New York) Commodity Component Demand Component

Reservation Fee Component

CONNECTICUT NATURAL GAS

(ALBERTA NORTHEAST GAS (PROGAS)) AVG Daily Quantity

Total Price (Waddington, New York Commodity Component

Demand Component

Reservation Fee Component

CONNECTICUT NATURAL GAS

(ALBERTA NORTHEAST GAS (ATCOR))

AVG Daily Quantity

Total Price (Waddington New York)

Commodity Component

Demand Component

Reservation Fee Component

CONNECTICUT NATURAL GAS

(ALBERTA NE GAS (AEC OIL \& GAS COMPANY))

AVG Daily Quantity

Total Price (Waddington, New York)

Commodity Component

Demand Component

Reservation Fee Component

CONSOLIDATED EDISON COMPANY OF N.Y.

(ALBERTA NE GAS (WGM LTD. 1))

AVG Daily Quantity

Total Price (Waddington, New York)

Commodity Component

Demand Component

Reservation Fee Component

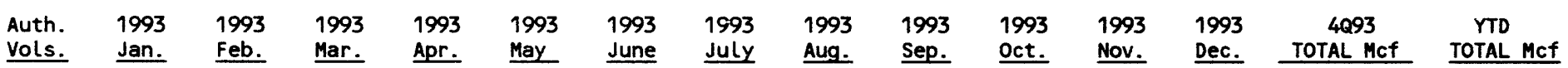

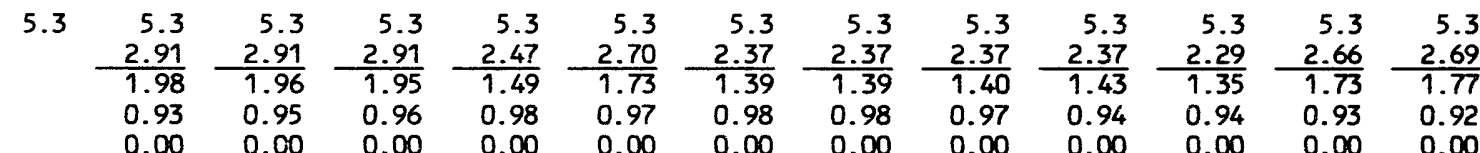

$483,000 \quad 1,921,500$

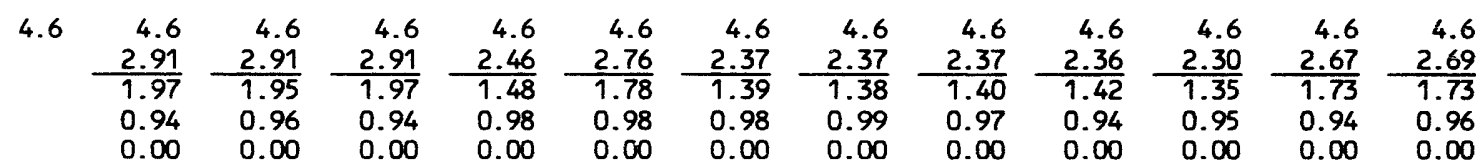

$425,040 \quad 1,690,951$

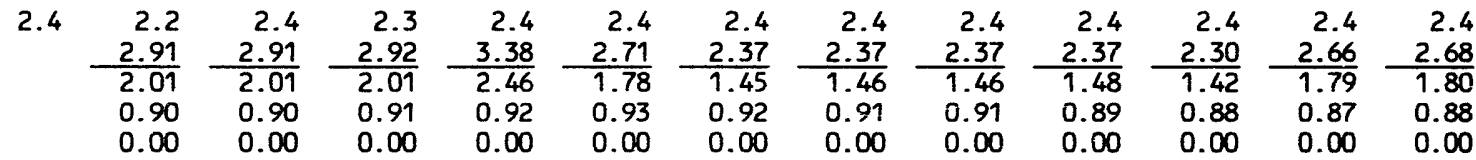

223,008

875,677

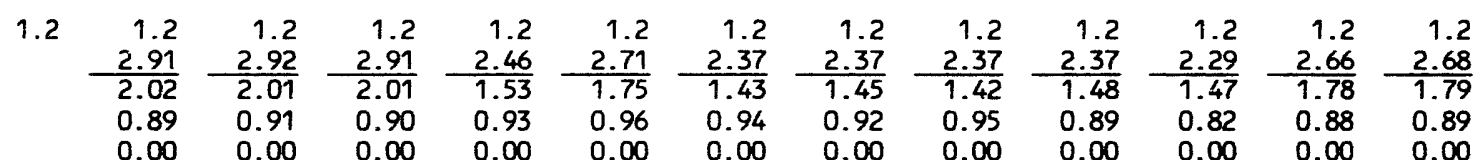

110,952

441,365

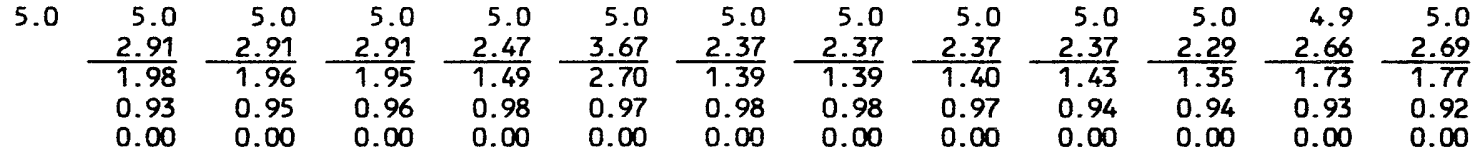




\section{VOLUNE AND PRICE REPORT}

\section{Long Term Imports}

Volumes (MMCf/d) \& Prices ( $\$$ /MMBTU) of Natural

Gas Imported During the Past 12 Months $01 / 01 / 93^{\text {Nat }}$ - 12/31/93

Long-Term Importer

CONSOLIDATED EDISON COMPANY OF N.Y.

(ALBERTA NE GAS (WGM LTD. 2))

AVG Daily Quantity

Total Price (Waddington, New York) Commodity Component

Demand Component

Reservation Fee Component

CONSOLIDATED EDISON COMPANY OF N.Y.

(ALBERTA NE GAS (PROGAS))

AVG Daily Quantity

Total Price (Waddington, New York) Commodity Component

Demand Component

Reservation Fee Component

CONSOLIDATED EDISON COMPANY OF N.Y.

(ALBERTA NE GAS (ATCOR))

AVG Daily Quantity

Total Price (Waddington, New York)

\section{Commodity Component}

Demand Component

Reservation Fee Component

CONSOLIDATED EDISON COMPANY OF N.Y.

(ALBERTA NE GAS (AEC OIL \& GAS))

AVG Daily Quantity

Total Price (Waddington, New York)

Commodity Component

Demand Component

Reservation Fee Component

CONSOLIDATED EDISON COMPANY OF NEW YORK

(AMOCO CANADA)

AVG Daily Quantity

Total Price (Niagara Falls, New York)

Commodity Component

Demand Component

Reservation Fee Component

$\begin{array}{lllllllllllll}\text { Auth. } & 1993 & 1993 & 1993 & 1993 & 1993 & 1993 & 1993 & 1993 & 1993 & 1993 & 1993 & 1993\end{array}$

vols. Jan. Feb. Mar. Apr. May June July Aug. Sep. Oct. Nov. Dec.

6

$$
\frac{1}{1.9}
$$

5.3

\begin{tabular}{lrrr}
5.3 & 5.3 & 5.3 & 5.3 \\
2.91 & 2.91 & 2.91 & 2.46 \\
\hline 1.97 & 1.95 & 1.97 & 1.48 \\
0.94 & 0.96 & 0.94 & 0.98 \\
0.00 & 0.00 & 0.00 & 0.00
\end{tabular}

2.6

$$
\frac{2.6}{2.01}
$$

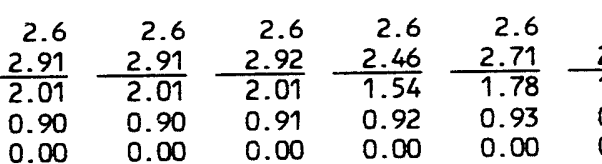

$$
\begin{array}{r}
5.3 \\
2.76 \\
\hline 1.78
\end{array}
$$

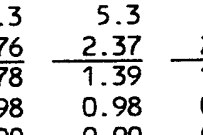$$
\frac{5.3}{1.38}
$$$$
2.37
$$$$
\begin{array}{rrrrr}
3 & 5.3 & 5.3 & 5.3 & 5.3 \\
& 2.36 & 2.30 & 2.67 & 2.69 \\
\cline { 5 - 5 } & 1.42 & 1.35 & 1.73 & 1.73 \\
7 & 0.94 & 0.95 & 0.94 & 0.96 \\
0 & 0.00 & 0.00 & 0.00 & 0.00
\end{array}
$$

1.1
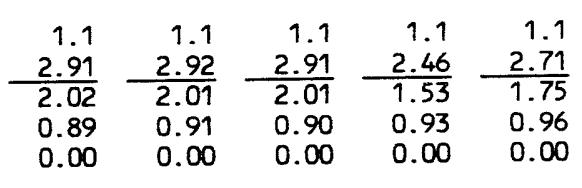

$$
\begin{array}{r}
1.1 \\
2.37 \\
\hline 1.43 \\
0.94 \\
0.00
\end{array}
$$$$
\begin{aligned}
& 1.1 \\
& 2.37 \\
& 1.45 \\
& 0.92 \\
& 0.00
\end{aligned}
$$

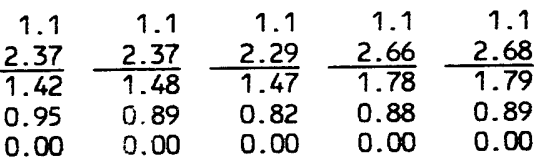

30.6

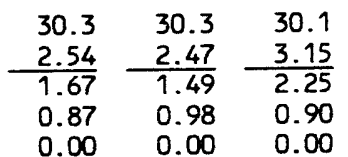

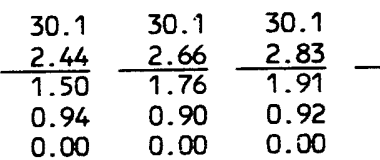

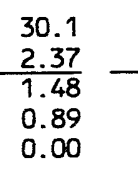

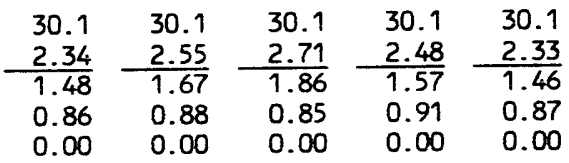

4093

TOTAL MCF TOTAL MC

$552,000 \quad 2,196,000$

$485,760 \quad 1,932,480$

240,212

955,596

101,935

405,801

$2,768,996 \quad 11,031,894$ 
VOLUNE AND PRICE REPORT

Long Term Imports

Volumes (MMCf/d) \& Prices (S/MMBTU) of Natural

Gas Imported During the Past 12 Months 01/01/93 - 12/31/93

\begin{tabular}{|c|c|c|c|c|c|c|c|c|c|c|c|c|c|c|c|}
\hline Long-Term Importer & $\begin{array}{l}\text { Auth. } \\
\text { Vols. }\end{array}$ & $\begin{array}{l}1993 \\
\text { Jan. }\end{array}$ & $\begin{array}{l}1993 \\
\text { Feb. }\end{array}$ & $\begin{array}{l}1993 \\
\text { Mar. }\end{array}$ & $\begin{array}{l}1993 \\
\text { Apr. }\end{array}$ & $\begin{array}{l}1993 \\
\text { May } \\
\end{array}$ & $\begin{array}{l}1993 \\
\text { June } \\
\end{array}$ & $\begin{array}{l}1993 \\
\text { July } \\
\end{array}$ & $\begin{array}{l}1993 \\
\text { Aug. }\end{array}$ & $\begin{array}{l}1993 \\
\text { Sep. }\end{array}$ & $\begin{array}{l}1993 \\
\text { Oct. }\end{array}$ & $\begin{array}{l}1993 \\
\text { Nov. } \\
\end{array}$ & $\begin{array}{l}1993 \\
\text { Dec. }\end{array}$ & $\begin{array}{c}4093 \\
\text { TOTAL MCf } \\
\end{array}$ & $\begin{array}{c}\text { YTD } \\
\text { TOTAL MCf }\end{array}$ \\
\hline $\begin{array}{l}\text { CONSUMERS POWER COMPANY } \\
\text { (WGM LTD.) } \\
\text { AVG Daily Quantity } \\
\text { Total Price (Noyes, Minnesota) } \\
\text { Commodity Component } \\
\text { Demand Component } \\
\text { Reservation Fee Component }\end{array}$ & 15.0 & $\begin{array}{l}15.0 \\
1.98 \\
1.54 \\
0.44 \\
0.00\end{array}$ & $\begin{array}{l}11.2 \\
2.22 \\
1.52 \\
0.70 \\
0.00\end{array}$ & $\begin{array}{l}13.5 \\
2.08 \\
1.56 \\
0.52 \\
0.00\end{array}$ & $\begin{array}{l}15.0 \\
2.04 \\
1.55 \\
0.49 \\
0.00\end{array}$ & $\begin{array}{l}15.0 \\
2.03 \\
1.57 \\
0.46 \\
0.00\end{array}$ & $\begin{array}{l}15.0 \\
2.02 \\
1.52 \\
0.50 \\
0.00\end{array}$ & $\begin{array}{l}15.0 \\
1.99 \\
1.51 \\
0.48 \\
0.00\end{array}$ & $\begin{array}{l}15.0 \\
2.00 \\
1.54 \\
0.46 \\
0.00\end{array}$ & $\begin{array}{l}15.0 \\
1.70 \\
1.24 \\
0.46 \\
0.00\end{array}$ & $\begin{array}{l}15.0 \\
1.67 \\
1.21 \\
0.46 \\
0.00\end{array}$ & $\begin{array}{l}15.0 \\
1.68 \\
1.21 \\
0.47 \\
0.00\end{array}$ & $\begin{array}{l}15.0 \\
1.69 \\
1.26 \\
0.43 \\
0.00\end{array}$ & $1,380,000$ & $5,336,663$ \\
\hline $\begin{array}{l}\text { CONSUMERS POWER COMPANY1 } \\
\text { (NORCEN ENERGY RESOURCES LTD.) } \\
\text { AVG Dai ly Quant ity } \\
\text { Total Price (Noyes, Minnesota) } \\
\text { Commodity Component } \\
\text { Demand Component } \\
\text { Reservation Fee Component }\end{array}$ & 14.0 & $\begin{array}{l}14.0 \\
2.07 \\
1.64 \\
0.43 \\
0.00\end{array}$ & $\begin{array}{r}10.5 \\
2.28 \\
1.65 \\
0.63 \\
0.00\end{array}$ & $\begin{array}{l}12.6 \\
2.16 \\
1.68 \\
0.48 \\
0.00\end{array}$ & $\begin{array}{l}14.0 \\
2.13 \\
1.67 \\
0.46 \\
0.00\end{array}$ & $\begin{array}{l}14.0 \\
2.12 \\
1.69 \\
0.43 \\
0.00\end{array}$ & $\begin{array}{l}14.0 \\
2.11 \\
1.66 \\
0.45 \\
0.00\end{array}$ & $\begin{array}{l}14.0 \\
2.09 \\
1.66 \\
0.43 \\
0.00\end{array}$ & $\begin{array}{l}14.0 \\
2.09 \\
1.66 \\
0.43 \\
0.00\end{array}$ & $\begin{array}{l}14.0 \\
1.79 \\
1.36 \\
0.43 \\
0.00\end{array}$ & $\begin{array}{l}14.0 \\
1.76 \\
1.34 \\
0.42 \\
0.00\end{array}$ & $\begin{array}{l}14.0 \\
1.77 \\
1.33 \\
0.44 \\
0.00\end{array}$ & $\begin{array}{l}14.0 \\
1.77 \\
1.35 \\
0.42 \\
0.00\end{array}$ & $1,287,922$ & $4,980,872$ \\
\hline $\begin{array}{l}\text { CONSUMERS POWER COMPANY2 } \\
\text { (HUSKY OIL) } \\
\text { AVG Daily Quantity } \\
\text { Total Price (Noyes, Minnesota) } \\
\text { Commodity Component } \\
\text { Demand Component } \\
\text { Reservation Fee Component }\end{array}$ & 15.0 & $\begin{array}{l}15.0 \\
2.02 \\
1.60 \\
0.42 \\
0.00\end{array}$ & $\begin{array}{r}11.2 \\
2.22 \\
1.61 \\
0.61 \\
0.00\end{array}$ & $\begin{array}{l}13.6 \\
2.10 \\
1.62 \\
0.48 \\
0.00\end{array}$ & $\begin{array}{l}15.0 \\
2.07 \\
1.64 \\
0.43 \\
0.00\end{array}$ & $\begin{array}{l}15.0 \\
2.07 \\
1.65 \\
0.42 \\
0.00\end{array}$ & $\begin{array}{l}15.0 \\
2.05 \\
1.62 \\
0.43 \\
0.00\end{array}$ & $\begin{array}{l}15.0 \\
2.04 \\
1.62 \\
0.42 \\
0.00\end{array}$ & $\begin{array}{l}15.0 \\
2.04 \\
1.63 \\
0.41 \\
0.00\end{array}$ & $\begin{array}{l}15.0 \\
1.74 \\
1.33 \\
0.41 \\
0.00\end{array}$ & $\begin{array}{l}15.0 \\
1.71 \\
1.31 \\
0.40 \\
0.00\end{array}$ & $\begin{array}{l}15.0 \\
1.72 \\
1.31 \\
0.41 \\
0.00\end{array}$ & $\begin{array}{l}15.0 \\
1.73 \\
1.33 \\
0.40 \\
0.00\end{array}$ & $1,379,916$ & $5,336,606$ \\
\hline $\begin{array}{l}\text { CONSUMERS POWER COMPANY3 } \\
\text { (SHELL CANADA LIMITED) } \\
\text { AVG Daily Quant ity } \\
\text { Total Price (Noyes, Minnesota) } \\
\text { Commodity Component } \\
\text { Demand Component } \\
\text { Reservation Fee Component }\end{array}$ & 15.0 & $\begin{array}{l}15.0 \\
1.99 \\
1.58 \\
0.41 \\
0.00\end{array}$ & $\begin{array}{l}11.2 \\
2.20 \\
1.59 \\
0.61 \\
0.00\end{array}$ & $\begin{array}{l}13.6 \\
2.06 \\
1.60 \\
0.46 \\
0.00\end{array}$ & $\begin{array}{l}15.0 \\
2.04 \\
1.61 \\
0.43 \\
0.00\end{array}$ & $\begin{array}{l}15.0 \\
2.04 \\
1.63 \\
0.41 \\
0.00\end{array}$ & $\begin{array}{l}15.0 \\
2.03 \\
1.61 \\
0.42 \\
0.00\end{array}$ & $\begin{array}{l}15.0 \\
2.02 \\
1.61 \\
0.41 \\
0.00\end{array}$ & $\begin{array}{l}15.0 \\
2.01 \\
1.61 \\
0.40 \\
0.00\end{array}$ & $\begin{array}{l}15.0 \\
1.71 \\
1.31 \\
0.40 \\
0.00\end{array}$ & $\begin{array}{l}15.0 \\
1.68 \\
1.30 \\
0.38 \\
0.00\end{array}$ & $\begin{array}{l}15.0 \\
1.69 \\
1.28 \\
0.41 \\
0.00\end{array}$ & $\begin{array}{l}15.0 \\
1.70 \\
1.31 \\
0.39 \\
0.00\end{array}$ & $1,379,916$ & $5,336,646$ \\
\hline $\begin{array}{l}\text { CONSUMERS POWER COMPANY4 } \\
\text { (POCO PETROLEUMS, LTD.) } \\
\text { AVG Daily Quant ity } \\
\text { Total Price (Noyes, Minnesota) } \\
\text { Commodity Component } \\
\text { Demand Component } \\
\text { Reservation Fee Component }\end{array}$ & 15.0 & $\begin{array}{l}15.0 \\
2.06 \\
1.65 \\
0.41 \\
0.00\end{array}$ & $\begin{array}{r}11.2 \\
2.22 \\
1.62 \\
0.60 \\
0.00\end{array}$ & $\begin{array}{l}13.6 \\
2.07 \\
1.61 \\
0.46 \\
0.00\end{array}$ & $\begin{array}{l}15.0 \\
2.01 \\
1.59 \\
0.42 \\
0.00\end{array}$ & $\begin{array}{l}15.0 \\
1.99 \\
1.59 \\
0.40 \\
0.00\end{array}$ & $\begin{array}{l}15.0 \\
2.01 \\
1.59 \\
0.42 \\
0.00\end{array}$ & $\begin{array}{l}15.0 \\
1.98 \\
1.58 \\
0.40 \\
0.00\end{array}$ & $\begin{array}{l}15.0 \\
1.98 \\
1.59 \\
c .39 \\
0.00\end{array}$ & $\begin{array}{l}15.0 \\
1.67 \\
1.27 \\
0.40 \\
0.00\end{array}$ & $\begin{array}{l}15.0 \\
1.65 \\
1.27 \\
0.38 \\
0.00\end{array}$ & $\begin{array}{l}15.0 \\
1.67 \\
1.26 \\
0.41 \\
0.00\end{array}$ & $\begin{array}{l}15.0 \\
1.65 \\
1.26 \\
0.39 \\
0.00\end{array}$ & $1,380,256$ & $5,336,746$ \\
\hline
\end{tabular}




\section{VOLUNE AND PRICE REPORT}

Iong Term Imports

Volumes (MMcf/d) \& Prices ( $/$ MMBTU) of Natural

Gas Imported During the Past 12 Months $01 / 01 / 93-12 / 31 / 93$

Long-Term Importer

CONSUMERS POWER COMPANYS

(NORTH CANADIAN OILS, LTD.)

AVG Daily Quantity

Total Price (Noyes, Minnesota)

Demand Component

Reservation Fee Component

CRESTAR ENERGY MARKETING CORPORATION (1) (CRESTAR ENERGY)

AVG Daily Quantity

Total Price (Noyes, Minnesota)

Commodity Component

Demand Component

Reservation Fee Component

DARTMOUTH POWER ASSOCIATES, L.P.

(CANADIAN NATURAL GAS RESOURCES)

AVG Daily Quantity

Total Price (Waddington, New York) Commodity Compon

Reservation Fee Component

DARTMOUTH POWER ASSOCIATES, L.P.

(ANDERSON OIL \& GAS INC.)

AVG Daily Quantity

Total Price (Waddington, New York)

Commodity Component

Demand Component

Reservation Fee Component

\begin{tabular}{|c|c|c|c|c|c|c|c|c|c|c|c|c|}
\hline $\begin{array}{l}\text { Auth. } \\
\text { Vols. }\end{array}$ & $\begin{array}{l}1993 \\
\text { Jan. }\end{array}$ & $\begin{array}{l}1993 \\
\text { Feb. }\end{array}$ & $\begin{array}{l}1993 \\
\text { Mar. }\end{array}$ & $\begin{array}{l}1993 \\
\text { Apr. }\end{array}$ & $\begin{array}{l}1993 \\
\text { May }\end{array}$ & $\begin{array}{l}1993 \\
\text { June }\end{array}$ & $\begin{array}{r}1993 \\
\text { July } \\
\end{array}$ & $\begin{array}{l}1993 \\
\text { Aug. }\end{array}$ & $\begin{array}{l}1993 \\
\text { Sep. }\end{array}$ & $\begin{array}{l}1993 \\
\text { Oct. }\end{array}$ & $\begin{array}{l}1993 \\
\text { Nov. }\end{array}$ & $\begin{array}{l}1993 \\
\text { Dec. }\end{array}$ \\
\hline 10.0 & $\begin{array}{l}10.0 \\
1.97 \\
1.57 \\
0.40 \\
0.00\end{array}$ & $\begin{array}{r}7.0 \\
2.17 \\
1.52 \\
0.65 \\
0.00\end{array}$ & $\begin{array}{r}8.8 \\
2.02 \\
1.55 \\
0.47 \\
0.00\end{array}$ & $\begin{array}{l}10.0 \\
1.97 \\
1.55 \\
0.42 \\
0.00\end{array}$ & $\begin{array}{l}10.0 \\
1.99 \\
1.59 \\
0.40 \\
0.00\end{array}$ & $\begin{array}{l}10.0 \\
1.99 \\
1.57 \\
0.42 \\
0.00\end{array}$ & $\begin{array}{l}10.0 \\
1.97 \\
1.57 \\
0.40 \\
0.00\end{array}$ & $\begin{array}{l}10.0 \\
1.96 \\
1.57 \\
0.39 \\
0.00\end{array}$ & $\begin{array}{l}10.0 \\
1.67 \\
1.27 \\
0.40 \\
0.00\end{array}$ & $\begin{array}{l}10.0 \\
1.65 \\
1.26 \\
0.39 \\
0.00\end{array}$ & $\begin{array}{l}10.0 \\
1.66 \\
1.26 \\
0.40 \\
0.00\end{array}$ & $\begin{array}{l}10.0 \\
1.67 \\
1.28 \\
0.39 \\
0.00\end{array}$ \\
\hline 15.0 & $\begin{array}{r}0.0 \\
0.00 \\
0.00 \\
0.00 \\
0.00\end{array}$ & $\begin{array}{r}0.0 \\
0.00 \\
0.00 \\
0.00 \\
0.00\end{array}$ & $\begin{array}{r}0.0 \\
0.00 \\
0.00 \\
0.00 \\
0.00\end{array}$ & $\begin{array}{r}0.0 \\
0.00 \\
0.00 \\
0.00 \\
0.00\end{array}$ & $\begin{array}{r}0.0 \\
0.00 \\
0.00 \\
0.00 \\
0.00\end{array}$ & $\begin{array}{l}0.0 \\
0.00 \\
0.00 \\
0.00 \\
0.00\end{array}$ & $\begin{array}{r}0.0 \\
0.00 \\
0.00 \\
0.00 \\
0.00\end{array}$ & $\begin{array}{r}0.0 \\
0.00 \\
0.00 \\
0.00 \\
0.00\end{array}$ & $\begin{array}{l}0.0 \\
0.00 \\
0.00 \\
0.00 \\
0.00\end{array}$ & $\begin{array}{r}0.0 \\
0.00 \\
0.00 \\
0.00 \\
0.00\end{array}$ & $\begin{array}{l}14.9 \\
2.10 \\
1.61 \\
0.43 \\
0.06\end{array}$ & $\begin{array}{l}14.9 \\
2.54 \\
2.04 \\
0.42 \\
0.08\end{array}$ \\
\hline 5.0 & $\begin{array}{r}2.2 \\
2.78 \\
1.84 \\
0.94 \\
0.00\end{array}$ & $\begin{array}{r}4.9 \\
2.71 \\
1.77 \\
0.94 \\
0.00\end{array}$ & $\begin{array}{r}2.9 \\
2.62 \\
1.68 \\
0.94 \\
0.00\end{array}$ & $\begin{array}{r}2.9 \\
2.68 \\
1.71 \\
0.97 \\
0.00\end{array}$ & $\begin{array}{r}3.1 \\
2.67 \\
1.70 \\
0.97 \\
0.00\end{array}$ & $\begin{array}{r}1.7 \\
2.66 \\
1.69 \\
0.97 \\
0.00\end{array}$ & $\begin{array}{r}2.5 \\
2.76 \\
1.74 \\
1.02 \\
0.00\end{array}$ & $\begin{array}{r}3.8 \\
2.58 \\
1.74 \\
0.84 \\
0.00\end{array}$ & $\begin{array}{r}4.4 \\
2.45 \\
1.74 \\
0.71 \\
0.00\end{array}$ & $\begin{array}{r}4.0 \\
2.40 \\
1.55 \\
0.85 \\
0.00\end{array}$ & $\begin{array}{r}3.4 \\
2.41 \\
1.56 \\
0.85 \\
0.00\end{array}$ & $\begin{array}{r}2.0 \\
2.50 \\
1.65 \\
0.85 \\
0.00\end{array}$ \\
\hline 3.0 & $\begin{array}{r}0.1 \\
2.78 \\
1.84 \\
0.94 \\
0.00\end{array}$ & $\begin{array}{r}2.6 \\
2.71 \\
1.77 \\
0.94 \\
0.00\end{array}$ & $\begin{array}{l}1.7 \\
2.62 \\
1.68 \\
0.94 \\
0.00\end{array}$ & $\begin{array}{r}1.4 \\
2.68 \\
1.71 \\
0.97 \\
0.00\end{array}$ & $\begin{array}{r}1.8 \\
2.67 \\
1.70 \\
0.97 \\
0.00\end{array}$ & $\begin{array}{l}1.1 \\
2.66 \\
1.69 \\
0.97 \\
0.00\end{array}$ & $\begin{array}{r}1.8 \\
2.76 \\
1.74 \\
1.02 \\
0.00\end{array}$ & $\begin{array}{r}2.7 \\
2.58 \\
1.74 \\
0.84 \\
0.00\end{array}$ & $\begin{array}{r}2.9 \\
2.45 \\
1.74 \\
0.71 \\
0.00\end{array}$ & $\begin{array}{r}2.7 \\
2.40 \\
1.55 \\
0.85 \\
0.00\end{array}$ & $\begin{array}{r}2.3 \\
2.41 \\
1.56 \\
0.85 \\
0.00\end{array}$ & $\begin{array}{r}1.4 \\
2.50 \\
1.65 \\
0.85 \\
0.00\end{array}$ \\
\hline 2.0 & $\begin{array}{r}0.1 \\
2.78 \\
1.84 \\
0.94 \\
0.00\end{array}$ & $\begin{array}{l}1.8 \\
2.71 \\
1.77 \\
0.94 \\
0.00\end{array}$ & $\begin{array}{l}1.2 \\
2.62 \\
1.68 \\
0.94 \\
0.00\end{array}$ & $\begin{array}{r}0.9 \\
2.68 \\
1.71 \\
0.97 \\
0.00\end{array}$ & $\begin{array}{r}0.9 \\
2.67 \\
1.70 \\
0.97 \\
0.00\end{array}$ & $\begin{array}{r}0.6 \\
2.66 \\
1.69 \\
0.97 \\
0.00\end{array}$ & $\begin{array}{r}1.4 \\
2.76 \\
1.74 \\
1.02 \\
0.00\end{array}$ & $\begin{array}{r}1.6 \\
2.58 \\
1.74 \\
0.84 \\
0.00\end{array}$ & $\begin{array}{r}2.0 \\
2.45 \\
1.74 \\
0.71 \\
0.00\end{array}$ & $\begin{array}{r}1.4 \\
2.40 \\
1.55 \\
0.85 \\
0.00\end{array}$ & $\begin{array}{r}1.4 \\
2.41 \\
1.56 \\
0.85 \\
0.00\end{array}$ & $\begin{array}{r}1.0 \\
2.50 \\
1.65 \\
0.85 \\
0.00\end{array}$ \\
\hline
\end{tabular}

(EXCEL ENERGY INC.)

AVG Daily Quantity

Total Price (Waddington, New York)

Demodity Component

0.00

$\begin{array}{llll}0.00 & 0.00 & 0.00 & 0.00\end{array}$

.00
4093 TOTAL MCf TOTAL MCf

$919,945 \quad 3,537,267$

908,003

908,003

$290,367 \quad 1,152,804$

197,561

691,059

118,740

435,997

(1) Supply is sold to Northern States Power Company for retail customers in the Grand Forks and Fargo areas of North Dakota. 
VOLULE AND PRICE REPORT

Long Term Imports

Volumes (MMCf/d) \& Prices (S/MMBTU) of Natural

Gas Imported During the Past 12 Months $01 / 01 / 93$ - 12/31/93

\section{Long-Term Importer}

DARTMOUTH POWER ASSOCIATES, L.P.

(REMINGTON ENERGY LTD.)

AVG Daily Quantity

Total Price (Waddington, New York)

Commodity Component

Reservation Fee Component

DISTRIGAS CORPORATION (88-37-LNG)

(SONATRACH)

AVG Daily Quantity

Total Price (Everett, Massachusetts)

Commodity Component

Reservation Fee Component

DISTRIGAS CORPORATION (89-16-LNG)

(SONATRACH)

AVG Daily Quantity

Total Price (Everett, Massachusetts)

Commodity Component

Demand Component

Reservation Fee Component

DOME PETROLEUM CORPORATION (1)

(DOME PETROLEUM LIMITED)

AVG Daily Quant ity

Total Price (Noyes, Minnesota)

Commodity Component

Reservation Fee Component

ELIZABETHTOWN GAS COMPANY

(WGM LTD.)

AVG Daily Quant ity

Total Price (Niagara Falls, New York)

Commodity Component

Demand Component

Reservation fee Component

\begin{tabular}{|c|c|c|c|c|c|c|c|c|c|c|c|c|c|c|}
\hline $\begin{array}{l}\text { Auth. } \\
\text { Vols. }\end{array}$ & $\begin{array}{l}1993 \\
\text { Jan. }\end{array}$ & $\begin{array}{l}1993 \\
\text { feb. }\end{array}$ & $\begin{array}{l}1993 \\
\text { Mar. }\end{array}$ & $\begin{array}{l}1993 \\
\text { Apr. }\end{array}$ & $\begin{array}{l}1993 \\
\text { May }\end{array}$ & $\begin{array}{l}1993 \\
\text { June }\end{array}$ & $\begin{array}{l}1993 \\
\text { July } \\
\end{array}$ & $\begin{array}{l}1993 \\
\text { Aug. }\end{array}$ & $\begin{array}{l}1993 \\
\text { Sep. }\end{array}$ & $\begin{array}{l}1993 \\
\text { Oct. }\end{array}$ & $\begin{array}{l}1993 \\
\text { Nov. }\end{array}$ & $\begin{array}{l}1993 \\
\text { Dec. }\end{array}$ & $\begin{array}{c}4093 \\
\text { TOTAL MCf }\end{array}$ & $\begin{array}{c}\text { YTD } \\
\text { TOTAL MCf } \\
\end{array}$ \\
\hline 6.0 & $\begin{array}{r}0.3 \\
2.78 \\
1.84 \\
0.94 \\
0.00\end{array}$ & $\begin{array}{l}5.8 \\
2.71 \\
1.77 \\
0.94 \\
0.00\end{array}$ & $\begin{array}{r}3.5 \\
2.62 \\
1.68 \\
0.94 \\
0.00\end{array}$ & $\begin{array}{r}2.7 \\
2.68 \\
1.71 \\
0.97 \\
0.00\end{array}$ & $\begin{array}{r}3.3 \\
2.67 \\
1.70 \\
0.97 \\
0.00\end{array}$ & $\begin{array}{l}1.9 \\
2.66 \\
1.69 \\
0.97 \\
0.00\end{array}$ & $\begin{array}{r}3.8 \\
2.76 \\
1.74 \\
1.02 \\
0.00\end{array}$ & $\begin{array}{r}4.3 \\
2.58 \\
1.74 \\
0.84 \\
0.00\end{array}$ & $\begin{array}{r}5.9 \\
2.45 \\
1.74 \\
0.71 \\
0.00\end{array}$ & $\begin{array}{r}4.1 \\
2.40 \\
1.55 \\
0.85 \\
0.00\end{array}$ & $\begin{array}{r}3.8 \\
2.41 \\
1.56 \\
0.85 \\
0.00\end{array}$ & $\begin{array}{r}2.8 \\
2.50 \\
1.65 \\
0.85 \\
0.00\end{array}$ & 328,446 & $1,281,884$ \\
\hline 0.0 & $\begin{array}{l}82.9 \\
2.84 \\
2.84 \\
0.00 \\
0.00\end{array}$ & $\begin{array}{r}181.7 \\
2.84 \\
2.84 \\
0.00 \\
0.00\end{array}$ & $\begin{array}{r}0.0 \\
0.00 \\
0.00 \\
0.00 \\
0.00\end{array}$ & $\begin{array}{l}0.0 \\
0.00 \\
0.00 \\
0.00 \\
0.00\end{array}$ & $\begin{array}{r}0.0 \\
0.00 \\
0.00 \\
0.00 \\
0.00\end{array}$ & $\begin{array}{l}83.4 \\
1.73 \\
1.73 \\
0.00 \\
0.00\end{array}$ & $\begin{array}{r}0.0 \\
0.00 \\
0.00 \\
0.00 \\
0.00\end{array}$ & $\begin{array}{r}0.0 \\
0.00 \\
0.00 \\
0.00 \\
0.00\end{array}$ & $\begin{array}{l}87.2 \\
2.11 \\
2.11 \\
0.00 \\
0.00\end{array}$ & $\begin{array}{l}83.3 \\
2.21 \\
2.21 \\
0.00 \\
0.00\end{array}$ & $\begin{array}{l}83.1 \\
2.19 \\
2.19 \\
0.00 \\
0.00\end{array}$ & $\begin{array}{r}162.4 \\
2.22 \\
2.22 \\
0.00 \\
0.00\end{array}$ & $10,109,208$ & $23,066,160$ \\
\hline 0.0 & $\begin{array}{l}82.9 \\
2.18 \\
2.18 \\
0.00 \\
0.00\end{array}$ & $\begin{array}{r}0.0 \\
0.00 \\
0.00 \\
0.00 \\
0.00\end{array}$ & $\begin{array}{l}83.2 \\
2.17 \\
2.17 \\
0.00 \\
0.00\end{array}$ & $\begin{array}{r}171.5 \\
2.14 \\
2.14 \\
0.00 \\
0.00\end{array}$ & $\begin{array}{l}86.2 \\
2.08 \\
2.08 \\
0.00 \\
0.00\end{array}$ & $\begin{array}{l}83.1 \\
2.16 \\
2.16 \\
0.00 \\
0.00\end{array}$ & $\begin{array}{l}80.3 \\
2.13 \\
2.13 \\
0.00 \\
0.00\end{array}$ & $\begin{array}{l}80.5 \\
2.15 \\
2.15 \\
0.00 \\
0.00\end{array}$ & $\begin{array}{l}83.2 \\
2.11 \\
2.11 \\
0.00 \\
0.00\end{array}$ & $\begin{array}{l}80.4 \\
2.11 \\
2.11 \\
0.00 \\
0.00\end{array}$ & $\begin{array}{l}85.7 \\
2.11 \\
2.11 \\
0.00 \\
0.00\end{array}$ & $\begin{array}{r}0.0 \\
0.00 \\
0.00 \\
0.00 \\
0.00\end{array}$ & $5,064,779$ & $28,009,930$ \\
\hline 15.0 & $\begin{array}{l}14.8 \\
2.02 \\
1.64 \\
0.38 \\
0.00\end{array}$ & $\begin{array}{l}14.8 \\
1.86 \\
1.24 \\
0.62 \\
0.00\end{array}$ & $\begin{array}{l}14.8 \\
2.05 \\
1.49 \\
0.56 \\
0.00\end{array}$ & $\begin{array}{l}14.8 \\
2.27 \\
1.69 \\
0.58 \\
0.00\end{array}$ & $\begin{array}{r}9.8 \\
3.03 \\
2.19 \\
0.84 \\
0.00\end{array}$ & $\begin{array}{l}14.9 \\
2.03 \\
1.45 \\
0.58 \\
0.00\end{array}$ & $\begin{array}{l}14.8 \\
2.01 \\
1.45 \\
0.56 \\
0.00\end{array}$ & $\begin{array}{r}14.0 \\
2.14 \\
1.55 \\
0.59 \\
0.00\end{array}$ & $\begin{array}{l}14.8 \\
2.37 \\
1.79 \\
0.58 \\
0.00\end{array}$ & $\begin{array}{l}14.8 \\
1.54 \\
1.54 \\
0.00 \\
0.00\end{array}$ & $\begin{array}{r}0.0 \\
0.00 \\
0.00 \\
0.00 \\
0.00\end{array}$ & $\begin{array}{r}0.0 \\
0.00 \\
0.00 \\
0.00 \\
0.00\end{array}$ & 458,037 & $4,337,270$ \\
\hline 10.0 & $\begin{array}{l}10.0 \\
2.47 \\
1.42 \\
1.05 \\
0.00\end{array}$ & $\begin{array}{l}10.0 \\
2.64 \\
1.48 \\
1.16 \\
0.00\end{array}$ & $\begin{array}{r}9.9 \\
2.55 \\
1.48 \\
1.07 \\
0.00\end{array}$ & $\begin{array}{r}9.9 \\
2.93 \\
1.80 \\
1.13 \\
0.00\end{array}$ & $\begin{array}{l}10.0 \\
3.08 \\
2.00 \\
1.08 \\
0.00\end{array}$ & $\begin{array}{l}10.0 \\
2.90 \\
1.79 \\
1.11 \\
0.00\end{array}$ & $\begin{array}{r}9.7 \\
2.36 \\
1.25 \\
1.11 \\
0.00\end{array}$ & $\begin{array}{r}10.0 \\
2.68 \\
1.62 \\
1.06 \\
0.00\end{array}$ & $\begin{array}{r}9.9 \\
2.75 \\
1.66 \\
1.09 \\
0.00\end{array}$ & $\begin{array}{l}10.0 \\
2.76 \\
1.72 \\
1.04 \\
0.00\end{array}$ & $\begin{array}{l}10.0 \\
3.13 \\
2.06 \\
1.07 \\
0.00\end{array}$ & $\begin{array}{l}10.0 \\
3.01 \\
1.97 \\
1.04 \\
0.00\end{array}$ & 918,770 & $3,643,147$ \\
\hline
\end{tabular}

(1) This contract expired on 10/31/93; Crestar Energy Marketing Corporation replaces Dome as the importer. 


\section{VOLULE AND PRICE REPORT}

Long Term Imports

Volumes (MMCf/d) \& Prices (S/MMBTU) of Natural

Gas Imported During the Past 12 Months $01 / 01 / 93^{-} 12 / 31 / 93$

Long-Tern Importer

ENCOGEN FOUR PARTNERS, L.P.

(SCEPTRE RESOURCES LIMITED)

AVG Daily quantity

Total Price (Niagara Falls, New York)

\section{Comand compont}

Reservation Fee Component

ENCOGEN MORTHWEST, L.P. (2)

(CANWEST GAS SUPPLY, INC.)

AVG Daily Quantity

Total Price (Sumas, Washington)

\section{Cominodity Component}

Dewand Component

Reservation Fee Component

ENERGYNORTH, INC.

(ALBERTA NORTHEAST GAS (WGM LTD. 1))

AVG Daily Quantity

Total Price (Waddington, New York)

\section{Commodity Component}

Demand Component

Reservation Fee Component

$1993 \quad 1993 \quad 1993 \quad 1993$

\begin{tabular}{llllll}
1993 & 1993 & 1993 & 1993 & 1993 & 1993 \\
\hline
\end{tabular}

4093

Q93

YTD

vols. Jan. Feb. Mar. Apr. May June July Aug

15.6

$\begin{array}{lrlllll}.6 & 0.0 & 0.0 & 0.0 & 0.0 & 0.0 & 0.0 \\ 0.00 & 0.00 & 0.00 & 0.00 & 0.00 & 0.00 \\ & 0.00 & 0.00 & 0.00 & 0.00 & 0.00 & 0.00 \\ 0.00 & 0.00 & 0.00 & 0.00 & 0.00 & 0.00 \\ 0.00 & 0.00 & 0.00 & 0.00 & 0.00 & 0.00\end{array}$

0.00
0.00
0.00

.00

$\begin{array}{llll}14.0 & 7.4 & 12.6 & 13.8\end{array}$

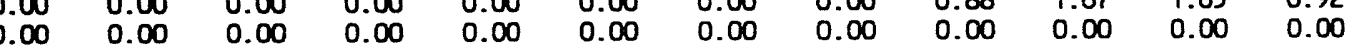

\begin{tabular}{|c|c|c|c|c|}
\hline $\begin{array}{r}0.0 \\
0.00 \\
0.00 \\
0.00 \\
0.00\end{array}$ & $\begin{array}{r}0.0 \\
0.00 \\
0.00 \\
0.00 \\
0.00\end{array}$ & $\begin{array}{r}0.0 \\
0.00 \\
0.00 \\
0.00 \\
0.00\end{array}$ & $\begin{array}{r}0.0 \\
0.00 \\
0.00 \\
0.00 \\
0.00\end{array}$ & $\begin{array}{l}0.0 \\
0.00 \\
0.00 \\
0.00 \\
0.00\end{array}$ \\
\hline
\end{tabular}

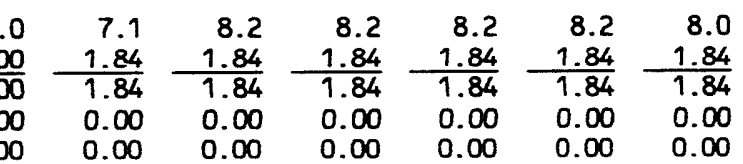

4.0

\begin{tabular}{|c|c|c|c|c|c|c|c|c|c|c|c|}
\hline $\begin{array}{r}3.9 \\
2.91 \\
1.98 \\
0.93 \\
0.00\end{array}$ & $\begin{array}{l}3.9 \\
2.91 \\
1.96 \\
0.95 \\
0.00\end{array}$ & $\begin{array}{r}3.9 \\
2.91 \\
1.95 \\
0.96 \\
0.00\end{array}$ & $\begin{array}{r}3.9 \\
2.47 \\
1.49 \\
0.98 \\
0.00\end{array}$ & $\begin{array}{l}3.9 \\
2.70 \\
1.73 \\
0.97 \\
0.00\end{array}$ & $\begin{array}{r}3.9 \\
2.37 \\
1.39 \\
0.98 \\
0.00\end{array}$ & $\begin{array}{r}3.9 \\
2.37 \\
1.39 \\
0.98 \\
0.00\end{array}$ & $\begin{array}{l}3.9 \\
2.37 \\
1.40 \\
0.97 \\
0.00\end{array}$ & $\begin{array}{l}3.9 \\
2.37 \\
1.43 \\
0.94 \\
0.00\end{array}$ & $\begin{array}{r}3.9 \\
2.29 \\
1.35 \\
0.94 \\
0.00\end{array}$ & $\begin{array}{r}4.0 \\
2.66 \\
1.73 \\
0.93 \\
0.00\end{array}$ & $\begin{array}{r}3.9 \\
2.69 \\
1.77 \\
0.92 \\
0.00\end{array}$ \\
\hline
\end{tabular}

SSEX COUNTY GAS CO

(ALBERTA NORTHEAST GAS (WGM LTD. 1))

AVG Daily Quantity

Total Price (Waddington, New York)

\section{Commodity component}

\section{Comand Component}

Reservation Fee component

FULTON COGENERATION ASSOCIATES

(STAR OIL \& GAS LIMITED)

AVG Daily Quantity

Total Price (Niagara Falls, New York)

Commodity Component

Dewand Component

Reservation fee component \begin{tabular}{lrlllllllllllll}
2.0 & 2.0 & 2.0 & 2.0 & 2.0 & 2.0 & 2.0 & 2.0 & 2.0 & 2.0 & 2.0 & 2.0 & 2.0 \\
& 2.91 & 2.91 & 2.91 & 2.47 & 2.70 & 2.37 & 2.37 & 2.37 & 2.37 & 2.29 & 2.66 & 2.69 \\
\hline & 1.98 & 1.96 & 1.95 & 1.49 & 1.73 & 1.39 & 1.39 & 1.40 & 1.43 & 1.35 & 1.73 & 1.77 \\
0.93 & 0.95 & 0.96 & 0.98 & 0.97 & 0.98 & 0.98 & 0.97 & 0.94 & 0.94 & 0.93 & 0.92 \\
0.00 & 0.00 & 0.00 & 0.00 & 0.00 & 0.00 & 0.00 & 0.00 & 0.00 & 0.00 & 0.00 & 0.00
\end{tabular}
181,499

720,349

$749,113 \quad 1,470,070$

$362,914 \quad 1,440,522$

$541,336 \quad 1,933,230$

(1) Supply used to fuel a 62 Megawatt gas-fired cogeneration facility located at Buffalo, N.Y.

(2) Supply used to fuel a 160 Megawatt gas-fired cogeneration facility located at Bellingham, WA. 


\section{VOLUNE AND PRICE REPORT}

\section{Long Term Imports}

Volumes (MMCf/d) \& Prices (S/MMBTU) of Natural

Gas Imported During the Past 12 Months $01 / 01 / 93-12 / 31 / 93$

\section{Long-Term Importer}

GRANITE STATE GAS TRANSMISSION, INC. (SHELL CANADA LIMITED)

AVG Daily Quantity

Total Price (North Troy, Vermont)

Commodity Component

Reservation Fee Component

GRANITE STATE GAS TRANSMISSION, INC.

(SHELL CAMADA LIMITED)

AVG Daily Quantity

Total Price (Haddington, New York)

Commodity Componen

Dewand Component

Reservation fee Component

GRANITE STATE GAS TRANSMISSION, INC.

(DIRECT ENERGY MARKETING LTD.)

AVG Daily Quantity

Total Price (North rroy, Vermont)

\section{Commodity Component}

\section{Demand Component}

Reservation Fee component

GREAT FALLS GAS COMPANY

(SHELL CANADA LIMITED)

AVG Daily Quantity

Total Price (Babb, Montana)

Commodity Componen

Demand Component

Reservation fee Component

IGI RESOURCES, INC.

(MOBIL OIL CANADA)

AVG Daily Quantity

Total Price (Sumas, Washington)

Compodity Component

Demand Component

Reservation fee Component

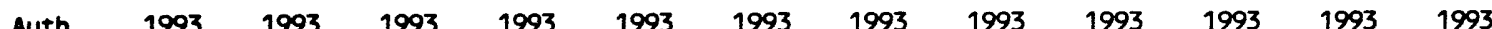

Vols. Jan. Feb. Mar. Apr. May June July Aug. Sep. oct. Nov. Dec.
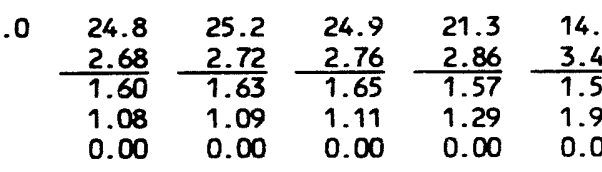

\begin{tabular}{l}
11.9 \\
3.83 \\
\hline 1.55 \\
2.28 \\
0.00
\end{tabular}

\begin{tabular}{l}
10.3 \\
4.03 \\
\hline 1.45 \\
2.58 \\
0.00
\end{tabular}

\begin{tabular}{l}
12.7 \\
3.45 \\
\hline 1.40 \\
2.05
\end{tabular}

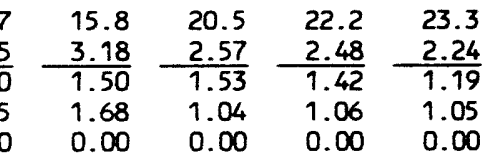

\begin{tabular}{lrr}
34.0 & 34.3 & 33.9 \\
2.66 & 2.56 & $\frac{2.72}{1.81}$ \\
\hline 1.77 & 1.66 & 0.91 \\
0.89 & 0.90 & 0.00 \\
0.00 & 0.00 & 0.00
\end{tabular}

\begin{tabular}{l}
34.3 \\
2.78 \\
\hline 1.86 \\
0.92 \\
0.00
\end{tabular}

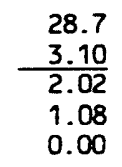

$\begin{array}{r}22.6 \\ 3.02 \\ \hline 1.65\end{array}$

\begin{tabular}{l}
20.6 \\
3.05 \\
\hline 1.56
\end{tabular}

32.3 \begin{tabular}{lrrrr}
33.7 & 31.4 & 34.6 & 34.6 \\
2.53 & 2.47 & 2.51 & 2.51 \\
\cline { 5 - 5 } & 1.64 & 1.61 & 1.63 & 1.64
\end{tabular}

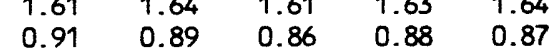

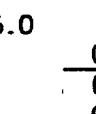

\begin{tabular}{cccccccccccc}
0.0 & 0.0 & 0.0 & 0.0 & 0.0 & 0.0 & 0.0 & 0.0 & 0.0 & 5.1 & 6.2 & 6.0 \\
0.00 & 0.00 & 0.00 & 0.00 & 0.00 & 0.00 & 0.00 & 0.00 & 0.00 & 2.59 & 2.50 & 2.45 \\
\hline 0.00 & 0.00 & 0.00 & 0.00 & 0.00 & 0.00 & 0.00 & 0.00 & 0.00 & 1.47 & 1.30 & $\frac{1.30}{1.30}$ \\
0.00 & 0.00 & 0.00 & 0.00 & 0.00 & 0.00 & 0.00 & 0.00 & 0.00 & 1.12 & 1.20 & 1.15 \\
0.00 & 0.00 & 0.00 & 0.00 & 0.00 & 0.00 & 0.00 & 0.00 & 0.00 & 0.00 & 0.00 & 0.00
\end{tabular}

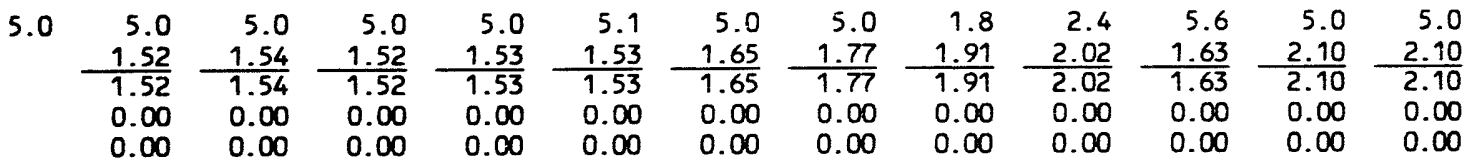

10.0

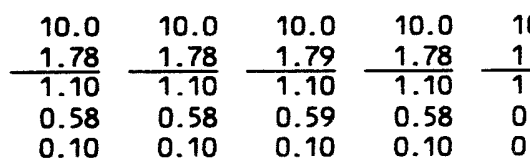

\begin{tabular}{llllllll}
.0 & 10.0 & 10.0 & 10.0 & 10.0 & 10.0 & 10.0 & 10.0 \\
10 & $\frac{1.77}{10}$ & $\frac{1.77}{1.10}$ & $\frac{1.76}{1.10}$ & $\frac{1.75}{1.10}$ & $\frac{1.75}{1.10}$ & $\frac{1.96}{1.23}$ & $\frac{1.62}{0.85}$ \\
\hline 58 & 0.57 & 0.57 & 0.56 & 0.55 & 0.55 & 0.57 & 0.55 \\
10 & 0.10 & 0.10 & 0.10 & 0.10 & 0.10 & 0.16 & 0.22
\end{tabular}

4093

TOTAL MCF TOTAL MCf

$2,022,653 \quad 6,912,620$

$3,080,114 \quad 11,429,698$

531,209

531,209

47,999

$1,680,419$

920,000

$3,660,000$ 


\section{VOLUNE AND PRICE REPORT}

\section{Long Term Imports}

Volumes (MMCf/d) \& Prices (S/MMBTU) of Natural

Gas Imported During the Past 12 Months $01 / 01 / 93-12 / 31 / 93$

\section{Long-Term Importer}

INDECK ENERGY SERVICES OF OSWEGO, INC. (INDECK GAS SUPPLY CORP.)

AVG Daily Quantity

Total Price (Niagara Falls, New York) Commodity Componen

\section{Demand Component}

Reservation Fee Component

INDECK-YERKES ENERGY SERVICES, INC.

(INDECK GAS SUPPLY CORP.)

AVG Daily Quantity

Total Price (Niagara Falls, New York)

Commodity Component

Demand Component

Reservation Fee Component

INDECK-YERKES LIMITED PARTNERSHIP

(NORTHSTAR ENERGY CORPORATION)

AVG Daily Quantity

Total Price (Niagara Falls, New York)

Commodity Componen

Demand Component

Reservation Fee Component

KAMINE/BESICORP CARTHAGE L.P.

(RENAISSANCE ENERGY LTD.)

AVG Daily Quantity

Total Price (Waddington, New York)

Commodity Component

Demand Component

Reservation Fee Component

KAMINE/BESICORP NATURAL DAM L.P.

(NORTH CANADIAN MARKETING INC.)

AVG Daily Quantity

Total Price (Waddington, New York)

Commodity Componen

Demand Component

Reservation Fee Component

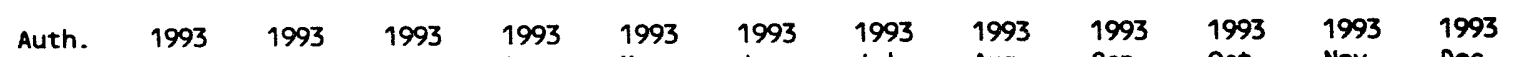

vols. Jan. Feb. Mar. Apr. May June suly Aug. $\underline{\text { Sep. }}$ oct. Nov. Dec.

12.3

\begin{tabular}{lrrr}
9.5 & 4.1 & 9.5 & 9.6 \\
2.52 & $\frac{2.53}{1.81}$ & $\frac{2.52}{1.81}$ & $\frac{2.48}{1.75}$ \\
\hline 1.81 & 0.72 & 0.71 & 0.73 \\
0.71 & 0.72 & \\
0.00 & 0.00 & 0.00 & 0.00
\end{tabular}

\begin{tabular}{r}
9.6 \\
2.47 \\
\hline 1.75 \\
0.72 \\
0.00
\end{tabular}

\begin{tabular}{r}
9.7 \\
2.47 \\
\hline 1.75 \\
0.72
\end{tabular}

$\frac{2.52}{1.81}$

$\begin{array}{rrrrr}9.7 & 9.7 & 9.7 & 9.5 & 9.5 \\ 2.52 & 2.52 & 2.51 & 2.51 & \frac{2.53}{1.81}\end{array}$

$\begin{array}{llllll}0.71 & 0.71 & 0.71 & 0.70 & 0.70 & 0.72\end{array}$

0.00

$0.00 \quad 0.00$

12.3

\begin{tabular}{rrrr}
9.3 & 9.3 & 9.3 & 9.3 \\
2.22 & 2.23 & 2.21 & 2.21 \\
\hline 1.50 & 1.51 & 1.50 & 1.48 \\
0.72 & 0.72 & 0.71 & 0.73 \\
0.00 & 0.00 & 0.00 & 0.00
\end{tabular}

9.39 .4

$\begin{array}{llllll}7.1 & 7.2 & 9.4 & 9.4 & 9.2 & 8.4\end{array}$

$\frac{2.17}{1.46} \frac{2.19}{1.47} \frac{2.33}{1.63} \frac{2.32}{1.62} \frac{2.20}{1.50} \frac{2.19}{1.50} \frac{2.27}{1.57} \frac{2.31}{1.61}$

0.00

$\begin{array}{llll}0.72 & 0.70 & 0.70 & 0.70\end{array}$

0.69

0.69
$0.70 \quad 0.70$

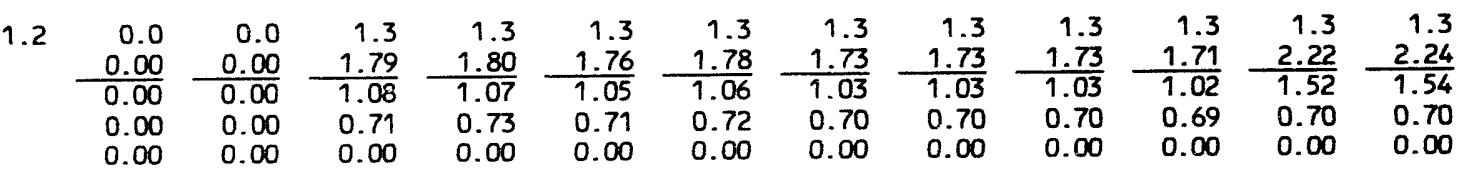

14.

\begin{tabular}{rrrrrrrrrrrrrr}
12.3 & 11.9 & 9.5 & 11.7 & 3.2 & 9.1 & 11.5 & 11.2 & 12.0 & 12.2 & 9.8 & 12.1 \\
2.72 & 2.83 & 2.89 & $\frac{2.59}{1.5}$ & $\frac{4.51}{1.81}$ & $\frac{2.91}{1.81}$ & $\frac{2.47}{1.77}$ & $\frac{2.47}{1.77}$ & $\frac{2.49}{1.76}$ & $\frac{2.61}{1.72}$ & $\frac{2.82}{1.74}$ & $\frac{2.51}{1.75}$ \\
\hline 1.96 & 1.96 & 1.96 & 1.82 & 1.81 & 1.70 & 0.70 & 0.70 & 0.73 & 0.89 & 1.08 & 0.76 \\
0.76 & 0.87 & 0.93 & 0.77 & 2.70 & 1.10 & 0.70 & 0.00 & 0.00 & 0.00 & 0.00 & 0.00 & 0.00
\end{tabular}

12.5

\begin{tabular}{cccc}
0.0 & 0.0 & 0.0 & 0.0 \\
0.00 & 0.00 & 0.00 & 0.00 \\
\cline { 3 - 4 } & 0.00 & 0.00 & 0.00 \\
0.00 & 0.00 & 0.00 & 0.00 \\
0.00 & 0.00 & 0.00 & 0.00
\end{tabular}

0.0
0.00
0.00
0.00
0.00 $\begin{array}{llll}0.0 & 0.0 & 0.0 & 0.0\end{array}$

0.

0.00
0.00
0.00 $\begin{array}{r}0.0 \\ 0.00 \\ \hline 0.00\end{array}$

$0.00 \quad 0.00$

0.00
0.00
0.00

$\begin{array}{lll}0.0 & 10.9 & 10.9\end{array}$

$\frac{0.00}{0.00} \frac{2.51}{1.76}-\frac{2.52}{1.76}$

$\begin{array}{lll}0.00 & 0.00 & 0.00\end{array}$ \begin{tabular}{cc}
4093 & YTD \\
TOTAL MCf & TOTAL MC \\
\hline
\end{tabular}

$877,417 \quad 3,353,788$

827,412

$3,246,615$

121,541

401,118

$1,047,210$

$3,853,001$

662,737

662,737 


\section{VOLUNE AND PRICE REPORT}

Volumes (MMCf/d) \& Prices (S/MMBTU) of Natural

Volumes (MMcf/d) \& Prices (\$/MMBTU) of Natural
Gas Imported During the Past 12 Months $01 / 01 / 93-12 / 31 / 93$

\section{Long-Term Importer}

KAMINE/BESICORP SOUTH GLENS FALLS L.P.

(RENAISSANCE ENERGY LTD.)

AVG Daily Quantity

Total Price (Noyes, Minnesota)

Commodity componen

Demand Component

KAMINE/BESICORP SYRACUSE L.P.

(NORTH CANADIAN MARKETING INC.)

AVG Daily Quantity

Total Price (Grand Island, New York)

Commodity Component

Demand Component

Reservation Fee Component

KCS ENERGY MARKETING, INC. (1)

(RAMARRO RESOURCES LTD.)

AVG Daily Quantity

Total Price (Niagara Falls, New York) Cemand component

Reservation Fee Component

LOCKPORT ENERGY ASSOCIATES, L.P.

(PROGAS LIMITED)

AVG Daily Quantity

Total Price (Niagara Falls, New York

Commodity Component

Demand Component

Reservation Fee Component

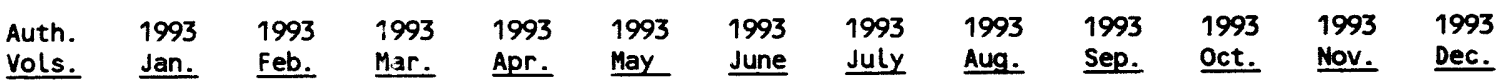

14.2

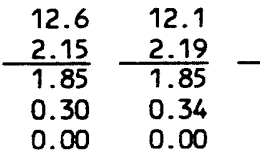

$11.4 \quad 12.6$

$\begin{array}{lllll}2.6 & 2.8 & 12.8 & 11.9 & 12.2\end{array}$

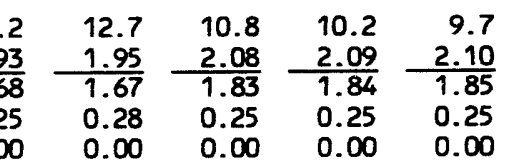

16.3

\begin{tabular}{lll}
0.0 & 0.0 & \\
0.00 & 0.00 & 0.0 \\
\hline 0.00 & 0.00 & 0.0 \\
0.00 & 0.00 & 0.0 \\
0.00 & 0.00 & 0.0
\end{tabular}

\begin{tabular}{cccccc}
0.0 & 0.0 & 0.0 & 0.0 & 0.0 & 0.0 \\
00 & 0.00 & 0.00 & 0.00 & 0.00 & 0.00 \\
\cline { 3 - 6 } & 0.00 & 0.00 & 0.00 & 0.00 & 0.00 \\
00 & 0.00 & 0.00 & 0.00 & 0.00 & 0.00 \\
00 & 0.00 & 0.00 & 0.00 & 0.00 & 0.00
\end{tabular}

\begin{tabular}{lrrr}
0.0 & 0.0 & 0.0 & 4.1 \\
0.00 & 0.00 & 0.00 & 4.65 \\
\cline { 2 - 4 } & 0.00 & 0.00 & 1.92 \\
0.00 & 0.00 & 0.00 & 2.73 \\
0.00 & 0.00 & 0.00 & 0.00
\end{tabular}

6.0

\begin{tabular}{rr}
5.9 & 5.8 \\
2.69 & 2.66 \\
\hline 1.99 & 1.84 \\
0.70 & 0.82
\end{tabular}

6.0
$\frac{2.63}{1.92}$

$\begin{array}{lllll}6.0 & 0.2 & 2.8 & 5.4 & 6.1\end{array}$

$0.00 \quad 0.00$

0.71
0.00

2.38

$\frac{23.70}{1.67}$

.23

1.93

$\frac{6.63}{1.95}$

6.06 .1

$\begin{array}{lll}6.1 & 6.0 & 6.0\end{array}$

$\begin{array}{llllll}1.93 & 1.95 & 1.93 & 1.82 & \frac{2.60}{1.90} & \frac{2.59}{1.90} \\ 0.68 & 0.68 & 0.72 & 0.68 & 0.70 & 0.69\end{array}$

0

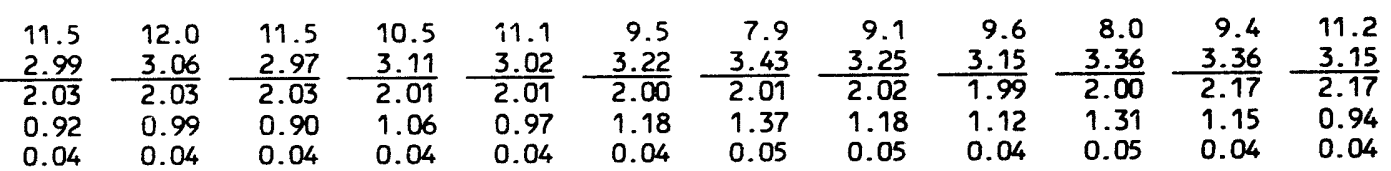

\begin{tabular}{|c|c|c|c|c|c|c|c|c|c|c|c|}
\hline $\begin{array}{l}30.4 \\
2.91 \\
1.98 \\
0.93 \\
0.00\end{array}$ & $\begin{array}{l}30.4 \\
2.91 \\
1.96 \\
0.95 \\
0.00\end{array}$ & $\begin{array}{l}30.4 \\
2.91 \\
1.95 \\
0.96 \\
0.00\end{array}$ & $\begin{array}{l}30.4 \\
2.47 \\
1.49 \\
0.98 \\
0.00\end{array}$ & $\begin{array}{l}30.4 \\
2.70 \\
1.73 \\
0.97 \\
0.00\end{array}$ & $\begin{array}{l}30.4 \\
2.37 \\
1.39 \\
0.98 \\
0.00\end{array}$ & $\begin{array}{l}30.4 \\
2.37 \\
1.39 \\
0.98 \\
0.00\end{array}$ & $\begin{array}{l}30.4 \\
2.37 \\
1.40 \\
0.97 \\
0.00\end{array}$ & $\begin{array}{l}30.4 \\
2.37 \\
1.43 \\
0.94 \\
0.00\end{array}$ & $\begin{array}{l}30.4 \\
2.29 \\
1.35 \\
0.94 \\
0.00\end{array}$ & $\begin{array}{l}30.4 \\
2.66 \\
1.73 \\
0.93 \\
0.00\end{array}$ & $\begin{array}{l}30.4 \\
2.69 \\
1.77 \\
0.92 \\
0.00\end{array}$ \\
\hline
\end{tabular}

4093

TOTAL MCf

940,505

$4,017,511$

126,957

126,957

LONG ISLAND LIGHTING COMPANY

(ALBERTA NORTHEAST GAS (WGM LTD. 1))

AVG Daily Quantity

Total Price (Waddington, New York) Commodity Component

Reservation Fee Component
30.4

-$$
0.00
$$

(1) Gas used to fuel a cogeneration facility in Milford, New Jersey. (Formerly Energy Marketing Exchange, Inc.) 


\section{VOLUME AND PRICE REPORT}

\section{Iong Term Imports}

Iong Term Imports
Volumes (MMCf/d) \& Prices ( $\$$ /MMBTU) of Natural

Gas Imported During the Past 12 Months $01 / 01 / 93-12 / 31 / 93$

\section{Long-Tern Inporter}

LONG ISLAND LIGHTING COMPANY

(ALBERTA NORTHEAST GAS (HGM LTO. 2))

AVG Daily Quantity

Total Price (Waddington, New York) Comadity Component

Demand Component

Reservation Fee Component

LONG ISLAND LIGHTING COMPANY

(ALBERTA MORTHEAST GAS (PROGAS))

AVG Daily Quantity

Total Price (Waddington, New York)

Commodity Component

Demand Component

Reservation Fee Component

LONG ISLAND LIGHTING COMPANY

(ALBERTA NORTHEAST GAS (ATCOR))

AVG Daily Quantity

Total Price (Waddington, New York) Comiod ity Component

Reservation fee component

LONG ISLAND LIGHTING COMPANY

(ALBERTA NE GAS (AEC OIL \& GAS)

AVG Daily Quantity

Total Price (Waddington, New York)

Commodity Component

Demand Component

Reservation Fee Component

MEGAN-RACINE ASSOCIATES, INC.

\section{(HGF LTD.)}

AVG Daily Quantity

Total Price (Massena, New York)

Demend compont

Demand Component
Reservation Fee Component

\begin{tabular}{|c|c|c|c|c|c|c|c|c|c|c|c|c|}
\hline $\begin{array}{l}\text { Auth. } \\
\text { Vols. }\end{array}$ & $\begin{array}{l}1993 \\
\text { Jan. }\end{array}$ & $\begin{array}{l}1993 \\
\text { Feb. }\end{array}$ & $\begin{array}{l}1993 \\
\text { Mar. }\end{array}$ & $\begin{array}{l}1993 \\
\text { Apr. }\end{array}$ & $\begin{array}{l}1993 \\
\text { May }\end{array}$ & $\begin{array}{l}1993 \\
\text { June } \\
\end{array}$ & $\begin{array}{l}1993 \\
\text { July }\end{array}$ & $\begin{array}{l}1993 \\
\text { Aug. }\end{array}$ & $\begin{array}{l}1993 \\
\text { Sep. }\end{array}$ & $\begin{array}{l}1993 \\
\text { Oct. }\end{array}$ & $\begin{array}{l}1993 \\
\text { Nov. }\end{array}$ & $\begin{array}{l}1993 \\
\text { Dec. }\end{array}$ \\
\hline 13.5 & $\begin{array}{l}13.5 \\
2.91 \\
1.98 \\
0.93 \\
0.00\end{array}$ & $\begin{array}{l}13.5 \\
2.91 \\
1.96 \\
0.95 \\
0.00\end{array}$ & $\begin{array}{l}13.5 \\
2.91 \\
1.95 \\
0.96 \\
0.00\end{array}$ & $\begin{array}{l}13.5 \\
2.47 \\
1.49 \\
0.98 \\
0.00\end{array}$ & $\begin{array}{l}13.5 \\
2.70 \\
1.73 \\
0.97 \\
0.00\end{array}$ & $\begin{array}{l}13.5 \\
2.37 \\
1.39 \\
0.98 \\
0.00\end{array}$ & $\begin{array}{l}13.5 \\
2.37 \\
1.39 \\
0.98 \\
0.00\end{array}$ & $\begin{array}{l}13.5 \\
2.37 \\
1.40 \\
0.97 \\
0.00\end{array}$ & $\begin{array}{l}13.5 \\
2.37 \\
1.43 \\
0.94 \\
0.00\end{array}$ & $\begin{array}{l}13.5 \\
2.29 \\
1.35 \\
0.94 \\
0.00\end{array}$ & $\begin{array}{l}13.5 \\
2.66 \\
1.73 \\
0.93 \\
0.00\end{array}$ & $\begin{array}{l}13.5 \\
2.69 \\
1.77 \\
0.92 \\
0.00\end{array}$ \\
\hline 11.9 & $\begin{array}{l}11.9 \\
2.91 \\
1.97 \\
0.94 \\
0.00\end{array}$ & $\begin{array}{l}11.9 \\
2.91 \\
1.95 \\
0.96 \\
0.00\end{array}$ & $\begin{array}{l}11.9 \\
2.91 \\
1.97 \\
0.94 \\
0.00\end{array}$ & $\begin{array}{l}11.9 \\
2.46 \\
1.48 \\
0.98 \\
0.00\end{array}$ & $\begin{array}{l}11.9 \\
2.76 \\
1.78 \\
0.98 \\
0.00\end{array}$ & $\begin{array}{l}11.9 \\
2.37 \\
1.39 \\
0.98 \\
0.00\end{array}$ & $\begin{array}{l}11.9 \\
2.37 \\
1.38 \\
0.99 \\
0.00\end{array}$ & $\begin{array}{l}11.9 \\
2.37 \\
1.40 \\
0.97 \\
0.00\end{array}$ & $\begin{array}{l}11.9 \\
2.36 \\
1.42 \\
0.94 \\
0.00\end{array}$ & $\begin{array}{r}11.9 \\
2.30 \\
1.35 \\
0.95 \\
0.00\end{array}$ & $\begin{array}{l}11.9 \\
2.67 \\
1.73 \\
0.94 \\
0.00\end{array}$ & $\begin{array}{l}11.9 \\
2.69 \\
1.73 \\
0.96 \\
0.00\end{array}$ \\
\hline 6.2 & $\begin{array}{r}6.2 \\
2.91 \\
2.01 \\
0.90 \\
0.00\end{array}$ & $\begin{array}{r}6.2 \\
2.91 \\
2.01 \\
0.90 \\
0.00\end{array}$ & $\begin{array}{l}6.2 \\
2.92 \\
2.01 \\
0.91 \\
0.00\end{array}$ & $\begin{array}{r}6.2 \\
2.46 \\
1.54 \\
0.92 \\
0.00\end{array}$ & $\begin{array}{l}6.2 \\
2.71 \\
1.78 \\
0.93 \\
0.00\end{array}$ & $\begin{array}{r}6.2 \\
2.37 \\
1.45 \\
0.92 \\
0.00\end{array}$ & $\begin{array}{r}6.2 \\
2.37 \\
1.46 \\
0.91 \\
0.00\end{array}$ & $\begin{array}{r}6.2 \\
2.37 \\
1.46 \\
0.91 \\
0.00\end{array}$ & $\begin{array}{r}6.2 \\
2.37 \\
1.48 \\
0.89 \\
0.00\end{array}$ & $\begin{array}{r}6.2 \\
2.30 \\
1.42 \\
0.88 \\
0.00\end{array}$ & $\begin{array}{r}6.2 \\
2.66 \\
1.79 \\
0.87 \\
0.00\end{array}$ & $\begin{array}{r}6.2 \\
2.68 \\
1.80 \\
0.88 \\
0.00\end{array}$ \\
\hline 3.1 & $\begin{array}{l}3.1 \\
2.91 \\
2.02 \\
0.89 \\
0.00\end{array}$ & $\begin{array}{r}3.1 \\
2.92 \\
2.01 \\
0.91 \\
0.00\end{array}$ & $\begin{array}{r}3.0 \\
2.91 \\
2.01 \\
0.90 \\
0.00\end{array}$ & $\begin{array}{r}3.1 \\
2.46 \\
1.53 \\
0.93 \\
0.00\end{array}$ & $\begin{array}{r}3.1 \\
2.71 \\
1.75 \\
0.96 \\
0.00\end{array}$ & $\begin{array}{r}3.1 \\
2.37 \\
1.43 \\
0.94 \\
0.00\end{array}$ & $\begin{array}{r}3.1 \\
2.37 \\
1.45 \\
0.92 \\
0.00\end{array}$ & $\begin{array}{r}3.1 \\
2.37 \\
1.42 \\
0.95 \\
0.00\end{array}$ & $\begin{array}{r}3.1 \\
2.37 \\
1.48 \\
0.89 \\
0.00\end{array}$ & $\begin{array}{r}3.1 \\
2.29 \\
1.47 \\
0.82 \\
0.00\end{array}$ & $\begin{array}{r}3.1 \\
2.66 \\
1.78 \\
0.88 \\
0.00\end{array}$ & $\begin{array}{r}3.1 \\
2.68 \\
1.79 \\
0.89 \\
0.00\end{array}$ \\
\hline 11.7 & $\begin{array}{r}8.1 \\
3.24 \\
1.72 \\
1.52 \\
0.00\end{array}$ & $\begin{array}{r}9.6 \\
3.14 \\
1.69 \\
1.45 \\
0.00\end{array}$ & $\begin{array}{l}10.0 \\
2.93 \\
1.69 \\
1.24 \\
0.00\end{array}$ & $\begin{array}{r}9.7 \\
2.79 \\
1.44 \\
1.35 \\
0.00\end{array}$ & $\begin{array}{r}9.4 \\
2.80 \\
1.44 \\
1.36 \\
0.00\end{array}$ & $\begin{array}{r}9.3 \\
2.85 \\
1.44 \\
1.41 \\
0.00\end{array}$ & $\begin{array}{r}5.3 \\
3.89 \\
1.50 \\
2.39 \\
0.00\end{array}$ & $\begin{array}{r}9.7 \\
2.80 \\
1.50 \\
1.30 \\
0.00\end{array}$ & $\begin{array}{l}10.4 \\
2.76 \\
1.50 \\
1.26 \\
0.00\end{array}$ & $\begin{array}{l}10.3 \\
2.82 \\
1.58 \\
1.24 \\
0.00\end{array}$ & $\begin{array}{r}9.2 \\
2.98 \\
1.58 \\
1.40 \\
0.00\end{array}$ & $\begin{array}{l}10.7 \\
2.74 \\
1.58 \\
1.16 \\
0.00\end{array}$ \\
\hline
\end{tabular}

4093 TOTAL MCf TOTAL MCf

$1,242,000$

$4,941,000$

$1,092,960$

$4,348,080$

566,260

$2,253,269$

281,980

$1,121,173$

$926,783 \quad 3,402,632$ 


\section{VOLUNE AND PRICE REPORT}

\section{Long Term Imports}

Volumes (MMCf/d) \& Prices ( $\$ / M M B T U$ ) of Natural

Gas Imported During the Past 12 Months $01 / 01 / 93$ - $12 / 31 / 93$

\section{Long-Term Importer}

MICHIGAN CONSOLIDATED GAS COMPANY

(WGM LTD.)

AVG Daily Quantity

Total Price (Noyes, Minnesota)

\section{Price (Noyes,}

Demand Component

Reservation Fee component

MIDLAND COGENERATION VENTURE?

(NORCEN ENERGY RESOURCES LTD.)

AVG Daity Quantity

Total Price (Noyes, Minnesota)

Comedity Component

Demand Component

Reservation Fee Component

MIDLAND COGENERATION VENTURE?

(SHELL CANADA LIMITED)

AVG Daily Quantity

Total Price (Noyes, Minnesota)

Commod ity Component

Reservation Fee Component

MIDLAND COGENERATION VENTURE3

(CANTERRA ENERGY LIMITED)

AVG Daily Quantity

Total Price (Noyes, Minnesota)

Commodity Component

Demand Component

Reservation Fee Component

MIDLAND COGENERATION VENTURE 4

(TRANSCANADA PIPELINES LIMITED)

AVG Daily Quantity

Total Price (Noyes, Minnesota)

Commodity Component

Demand Cumponent
Reservation Fee Component

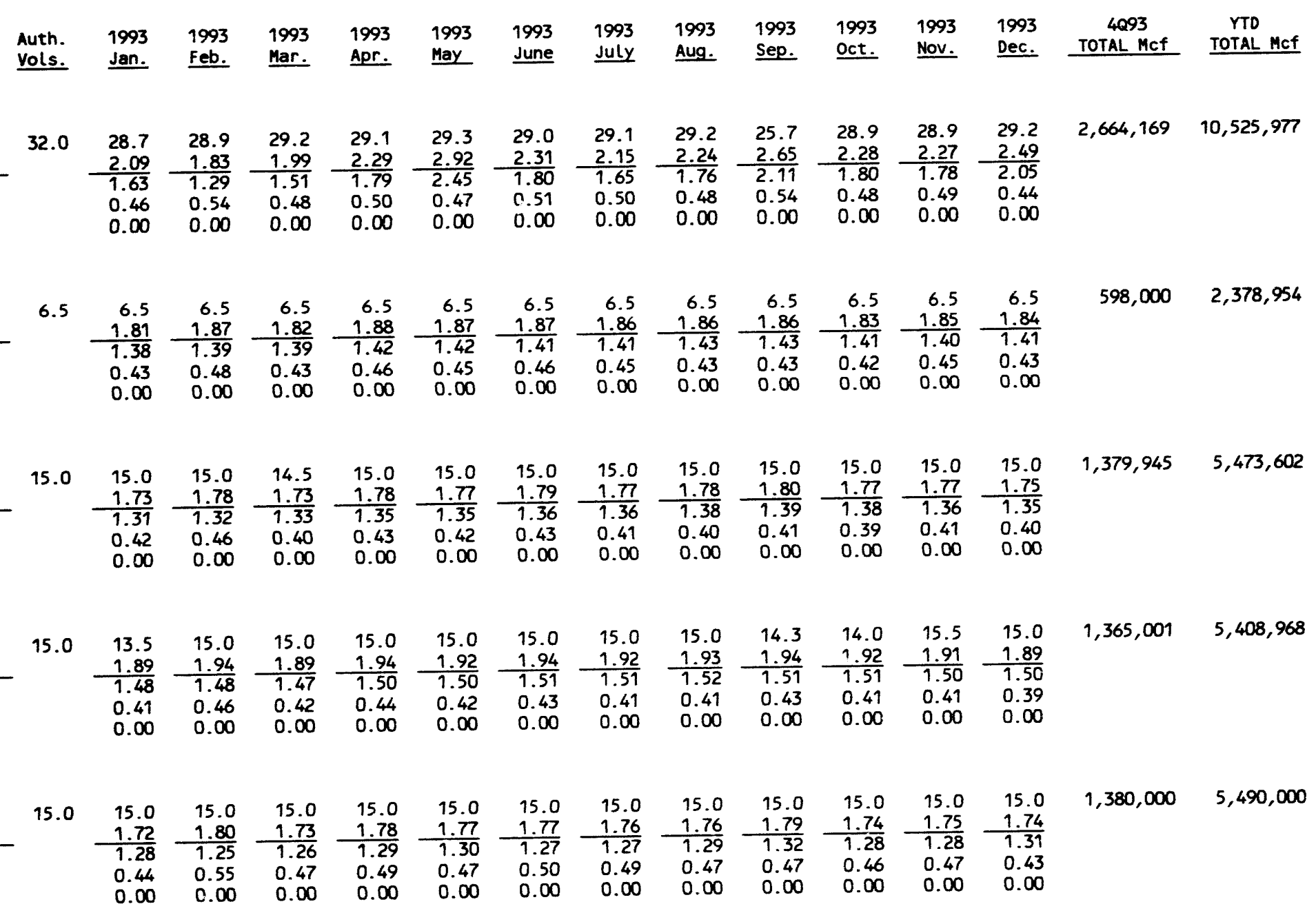




\section{VOLUNE AND PRICE REPORT}

Long Term Imports

Volumes (MMcf/d) \& Prices (S/MMBTU) of Natural

Gas Imported During the Past 12 Months $01 / 01 / 93-12 / 31 / 93$

\section{Long-Term Importer}

MIDLAND COGENERATION VENTURES (POCO PETROLEUMS, LTD.)

AVG Daily Quantity

Total Price (Noyes, Minnesota) Commodity Component

Demand Component

Reservation Fee Component

MIDLAND COGENERATION VENTURE6

(NORTH CANADIAN OILS, LTD.)

AVG Daily Quantity

Total Price (Noyes, Minnesota)

Comodity Component

Demand Component

Reservation Fee Component

MINNEGASCO, INC

(TRANSCANADA PIPELINES LIMITED)

AVG Daily Quantity

Total Price (Noyes, Minnesota)

Commodity Component

Reservation Fee Component

N.Y. STATE ELECTRIC \& GAS CO.

(ALBERTA NORTHEAST GAS (WGM LTD. 2))

AVG Daily Quantity

Total Price (Waddington, New York)

Commodity Component

Demand Component

Reservation Fee Component

N.Y. STATE ELECTRIC \& GAS CO

(ALBERTA NORTHEAST GAS (PROGAS))

AVG Daily Quantity

Total Price (Waddington, New York)

Commodity Combonent

Demand Component

Reservation Fee Component

\begin{tabular}{|c|c|c|c|c|c|c|c|c|c|c|c|c|c|c|}
\hline $\begin{array}{l}\text { Auth. } \\
\text { Vols. }\end{array}$ & $\begin{array}{l}1993 \\
\tan .\end{array}$ & $\begin{array}{l}1993 \\
\text { Feb. }\end{array}$ & $\begin{array}{l}1993 \\
\text { Mar. }\end{array}$ & $\begin{array}{l}1993 \\
\text { Apr. }\end{array}$ & $\begin{array}{l}1993 \\
\text { May }\end{array}$ & $\begin{array}{l}1993 \\
\text { June }\end{array}$ & $\begin{array}{l}1993 \\
\text { July }\end{array}$ & $\begin{array}{l}1993 \\
\text { Aug. }\end{array}$ & $\begin{array}{l}1993 \\
\text { Sep. }\end{array}$ & $\begin{array}{l}1993 \\
\text { oct. }\end{array}$ & $\begin{array}{l}1993 \\
\text { Nov. }\end{array}$ & $\begin{array}{l}1993 \\
\text { Dec. }\end{array}$ & $\begin{array}{c}4093 \\
\text { TOTAL MCf }\end{array}$ & $\begin{array}{c}\text { YTD } \\
\text { TOTAL MCf } \\
\end{array}$ \\
\hline 15.0 & $\begin{array}{l}15.0 \\
1.98 \\
1.58 \\
0.40 \\
0.00\end{array}$ & $\begin{array}{l}13.0 \\
1.87 \\
1.42 \\
0.45 \\
0.00\end{array}$ & $\begin{array}{l}15.0 \\
1.84 \\
1.43 \\
0.41 \\
0.00\end{array}$ & $\begin{array}{l}15.0 \\
1.89 \\
1.46 \\
0.43 \\
0.00\end{array}$ & $\begin{array}{l}15.0 \\
1.88 \\
1.47 \\
0.41 \\
0.00\end{array}$ & $\begin{array}{l}15.0 \\
2.32 \\
1.90 \\
0.42 \\
0.00\end{array}$ & $\begin{array}{l}15.0 \\
1.90 \\
1.49 \\
0.41 \\
0.00\end{array}$ & $\begin{array}{l}15.0 \\
1.91 \\
1.50 \\
0.41 \\
0.00\end{array}$ & $\begin{array}{l}15.0 \\
1.93 \\
1.53 \\
0.40 \\
0.00\end{array}$ & $\begin{array}{l}15.0 \\
1.51 \\
1.30 \\
0.21 \\
0.00\end{array}$ & $\begin{array}{l}15.0 \\
1.91 \\
1.50 \\
0.41 \\
0.00\end{array}$ & $\begin{array}{l}15.0 \\
1.90 \\
1.50 \\
0.40 \\
0.00\end{array}$ & $1,379,945$ & $5,489,780$ \\
\hline
\end{tabular}

10

\begin{tabular}{rrrrrrrrrrrrr}
5.2 & 5.0 & 5.0 & 10.0 & 10.0 & 10.0 & 10.0 & 10.0 & 7.1 & 9.0 & 10.0 & 8.4 \\
2.64 & 2.74 & 2.67 & 2.34 & $\frac{2.34}{1.34}$ & $\frac{2.36}{1.97}$ & $\frac{2.35}{1.98}$ & $\frac{2.37}{2.00}$ & $\frac{2.51}{1.98}$ & $\frac{2.38}{1.99}$ & $\frac{2.36}{1.99}$ & $\frac{2.42}{2.00}$ \\
\hline 1.90 & 1.71 & 1.91 & 1.95 & 1.96 & 1.97 & 0.37 & 0.37 & 0.53 & 0.39 & 0.37 & 0.42 \\
0.74 & 0.83 & 0.76 & 0.39 & 0.38 & 0.39 & 0.37 & 0.00 & 0.00 & 0.00 & 0.00 & 0.00 & 0.00 \\
0.00 & 0.00 & 0.00 & 0.00 & 0.00 & 0.00 & 0.00 & 0.00 &
\end{tabular}

50.0

\begin{tabular}{lllllllllllll}
49.3 & 49.2 & 49.3 & 34.5 & 19.1 & 19.7 & 19.7 & 19.6 & 19.7 & 34.9 & 49.3 & 49.3 \\
2.43 & 2.10 & 2.30 & $\frac{2.64}{2.04}$ & $\frac{3.15}{2.56}$ & $\frac{2.39}{1.80}$ & $\frac{2.63}{1.78}$ & $\frac{2.74}{1.89}$ & $\frac{3.01}{2.13}$ & $\frac{2.30}{1.88}$ & $\frac{2.39}{1.74}$ & $\frac{2.97}{2.34}$ \\
\hline 1.95 & 1.57 & 1.82 & 2.04 & 2.59 & 0.59 & 0.85 & 0.85 & 0.88 & 0.42 & 0.65 & 0.63 \\
0.34 & 0.38 & 0.34 & 0.50 & 0.59 & 0.59 & 0.00 & 0.00 & 0.00 & 0.00 & 0.00 & 0.00 \\
0.14 & 0.15 & 0.14 & 0.10 & 0.00 & 0.00 & 0.00 &
\end{tabular}

$4,086,936 \quad 12,598,331$

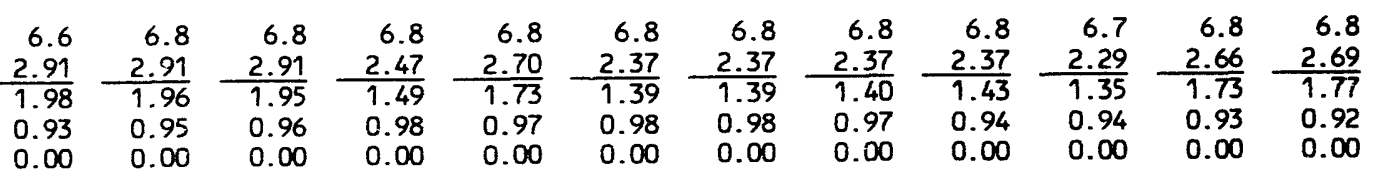

620,783

$2,465,903$

5.9

\begin{tabular}{rrrrrrrrrrrrr}
5.9 & 5.9 & 5.9 & 5.9 & 5.9 & 5.9 & 5.9 & 5.9 & 5.9 & 5.9 & 5.9 & 5.9 \\
2.91 & $\frac{2.91}{1.95}$ & $\frac{2.91}{1.97}$ & $\frac{2.46}{1.48}$ & $\frac{2.76}{1.78}$ & $\frac{2.37}{1.39}$ & $\frac{2.37}{1.38}$ & $\frac{2.37}{1.40}$ & $-\frac{2.36}{1.42}$ & $\frac{2.30}{1.35}$ & $\frac{2.67}{1.73}$ & $\frac{2.69}{1.73}$ \\
\hline 1.97 & 1.95 & 0.94 & 0.98 & 0.98 & 0.98 & 0.99 & 0.97 & 0.94 & 0.95 & 0.94 & 0.96 \\
0.94 & 0.96 & 0.94 & 0.00 & 0.00 & 0.00 & 0.00 & 0.00 & 0.00 & 0.00 & 0.00 & 0.00 \\
0.00 & 0.00 & 0.00 & 0.00 &
\end{tabular}

$546,294 \quad 2,173,793$ 


\section{VOLUNE AND PRICE REPORT
IONG Term Imports}

Volumes (MMCf/d) \& Prices (\$/MMBTU) of Natural

Gas Imported During the Past 12 Months $01 / 01 / 93^{-} 12 / 31 / 93$

\section{Long-Term Importer}

N.Y. STATE ELECTRIC \& GAS CO.

(ALBERTA NORTHEAST GAS (ATCOR))

AVG Daily Quantity

Total Price (Waddington, New York)

Commodity Component

Demand Component

Reservation fee Component

N.Y. STATE ELECTRIC \& GAS CO.

(ALBERTA NE GAS (AEC OIL \& GAS CO.))

AVG Daily Quantity

Total Price (Waddington, New York)

Commodity Component

Demand Component

Reservation Fee Component

N.Y. STATE ELECTRIC \& GAS CO. (1)

(PROGAS LIMITED)

AVG Daily Quantity

Total Price (Niagara Falls, New York)

Commodity Component

Demand Component

Reservation Fee Component

N.Y. STATE ELECTRIC \& GAS CO.

(CRESTAR ENERGY)

Total Price (Grand Island, New York)

Demand Component

Reservation Fee Component

NATIONAL FUEL GAS DISTRIBUTION CORPORATION

(ALBERTA NE GAS (WGM LTD))

AVG Daily Quantity

Total Price (Niagara Falls, New York

Commodity Component

Demand Component

Reservation Fee Component

\begin{tabular}{|c|c|c|c|c|c|c|c|c|c|c|c|c|}
\hline $\begin{array}{l}\text { Auth. } \\
\text { Vols. }\end{array}$ & $\begin{array}{l}1993 \\
\text { Jan. }\end{array}$ & $\begin{array}{l}1993 \\
\text { Feb. }\end{array}$ & $\begin{array}{l}1993 \\
\text { Mar. }\end{array}$ & $\begin{array}{l}1993 \\
\text { Apr. }\end{array}$ & $\begin{array}{l}1993 \\
\text { May }\end{array}$ & $\begin{array}{l}1993 \\
\text { June }\end{array}$ & $\begin{array}{l}1993 \\
\text { July }\end{array}$ & $\begin{array}{l}1993 \\
\text { Aug. }\end{array}$ & $\begin{array}{l}1993 \\
\text { Sep. }\end{array}$ & $\begin{array}{l}1993 \\
\text { oct. }\end{array}$ & $\begin{array}{l}1993 \\
\text { Nov. } \\
\end{array}$ & $\begin{array}{l}1993 \\
\text { Dec. }\end{array}$ \\
\hline 3.0 & $\begin{array}{r}3.0 \\
2.91 \\
2.01 \\
0.90 \\
0.00\end{array}$ & $\begin{array}{r}3.0 \\
2.91 \\
2.01 \\
0.90 \\
0.00\end{array}$ & $\begin{array}{r}2.8 \\
2.92 \\
2.01 \\
0.91 \\
0.00\end{array}$ & $\begin{array}{r}2.9 \\
2.46 \\
1.54 \\
0.92 \\
0.00\end{array}$ & $\begin{array}{r}2.9 \\
2.71 \\
1.78 \\
0.93 \\
0.00\end{array}$ & $\begin{array}{r}3.0 \\
2.37 \\
1.45 \\
0.92 \\
0.00\end{array}$ & $\begin{array}{l}3.0 \\
2.37 \\
1.46 \\
0.91 \\
0.00\end{array}$ & $\begin{array}{r}3.0 \\
2.37 \\
1.46 \\
0.91 \\
0.00\end{array}$ & $\begin{array}{r}3.0 \\
2.37 \\
1.48 \\
0.89 \\
0.00\end{array}$ & $\begin{array}{r}3.0 \\
2.30 \\
1.42 \\
0.88 \\
0.00\end{array}$ & $\begin{array}{r}3.0 \\
2.66 \\
1.79 \\
0.87 \\
0.00\end{array}$ & $\begin{array}{r}3.0 \\
2.68 \\
1.80 \\
0.88 \\
0.00\end{array}$ \\
\hline
\end{tabular}

1.

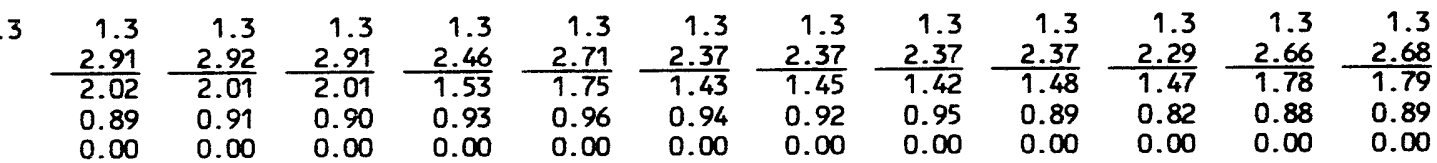

9.0

\begin{tabular}{|c|c|c|c|c|c|c|c|c|c|c|c|}
\hline $\begin{array}{r}0.0 \\
0.00 \\
0.00 \\
0.00 \\
0.00\end{array}$ & $\begin{array}{r}0.0 \\
0.00 \\
0.00 \\
0.00 \\
0.00\end{array}$ & $\begin{array}{r}0.0 \\
0.00 \\
0.00 \\
0.00 \\
0.00\end{array}$ & $\begin{array}{r}0.0 \\
0.00 \\
0.00 \\
0.00 \\
0.00\end{array}$ & $\begin{array}{r}0.0 \\
0.00 \\
0.00 \\
0.00 \\
0.00\end{array}$ & $\begin{array}{l}0.0 \\
0.00 \\
0.00 \\
0.00 \\
0.00\end{array}$ & $\begin{array}{r}0.0 \\
0.00 \\
0.00 \\
0.00 \\
0.00\end{array}$ & $\begin{array}{r}0.0 \\
0.00 \\
0.00 \\
0.00 \\
0.00\end{array}$ & $\begin{array}{r}0.0 \\
0.00 \\
0.00 \\
0.00 \\
0.00\end{array}$ & $\begin{array}{r}0.0 \\
0.00 \\
0.00 \\
0.00 \\
0.00\end{array}$ & $\begin{array}{r}0.0 \\
0.00 \\
0.00 \\
0.00 \\
0.00\end{array}$ & $\begin{array}{r}5.3 \\
2.39 \\
1.24 \\
1.15 \\
0.00\end{array}$ \\
\hline
\end{tabular}

10.0

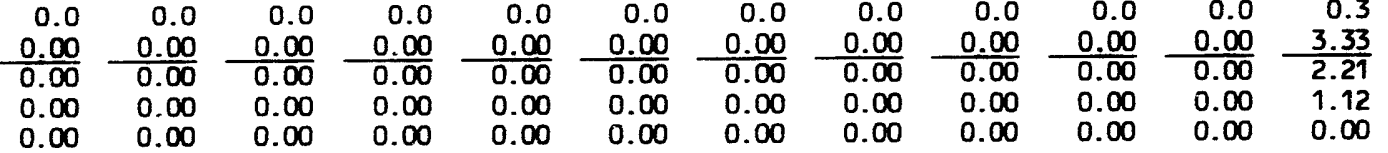

(2)

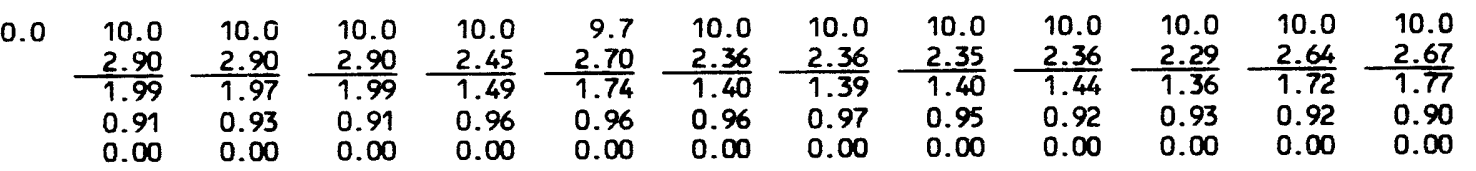

4093

TOTAL MCF TOTAL MCF

$274,435 \quad 1,080,083$

121,930

485,254

165,301

165,301

9,601

$920,000 \quad 3,650,003$

(1) NYSERG's volumes include 7,200 Mcf at Champlain, New York. Distribution Corporation. 


\section{VOLUNE AND PRICE REPORT}

\section{Long Term Imports}

Volumes (MMCf/d) \& Prices ( $\$$ /MMBTU) of Natural

Gas Imported During the Past 12 Months $01 / 01 / 93^{-} 12 / 31 / 93$

Long-Term Importer

NATURAL GAS PIPELINE COMPANY OF AMERICA

(PROGAS LIMITED)

AVG Daily Quantity

Total Price (Port of Morgan, Montana)

Commodity Component

Demand Component

Reservation Fee Component

NATURAL GAS PIPELINE COMPANY OF AMERICA

(WGM LTD.)

AVG Daily Quantity

Total Price (Noyes, Minnesota)

Commodity component

Demand Component

Reservation fee Component

NEW ENGLAND POWER COMPANY

(TALISMAN ENERGY INC.)

AVG Daily Quantity

Total Price (Waddington, New York)

Commodity Component

Demand Component

Reservation fee Component

NEW ENGLAND POWER COMPANY

(RENAISSANCE ENERGY LIMITED)

AVG Daily Quantity

Total Price (Waddington, New York)

Commodity Component

Demand Component

Reservation Fee Component

NEW ENGLAND POWER COMPANY

(SCEPTRE RESOURCES LIMITED)

AVG Daily Quantity

Total Price (Waddington, New York)

Commodity Component

Reservation Fee Component

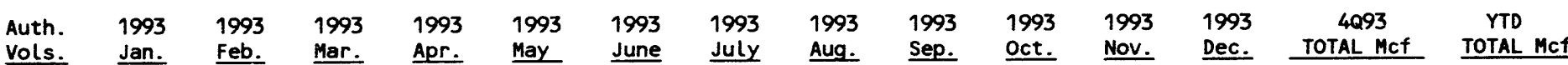

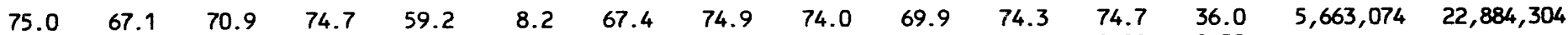
$\frac{1.85}{1.52} \frac{1.55}{1.20} \frac{1.75}{1.45} \frac{2.13}{1.73} \frac{4.99}{2.22} \frac{1.85}{1.49} \frac{1.76}{1.46} \frac{1.90}{1.60}-\frac{2.00}{1.69} \frac{1.80}{1.52} \frac{1.91}{1.60} \frac{2.50}{1.88}$

$\begin{array}{llllllllllll}0.33 & 0.35 & 0.30 & 0.40 & 2.77 & 0.36 & 0.30 & 0.30 & 0.31 & 0.28 & 0.31 & 0.62 \\ 0.00 & 0.00 & 0.00 & 0.00 & 0.00 & 0.00 & 0.00 & 0.00 & 0.00 & 0.00 & 0.00 & 0.00\end{array}$

$\begin{array}{lllllllllllll}171.3 & 75.0 & 148.5 & 171.3 & 165.6 & 9.9 & 155.1 & 171.3 & 171.3 & 139.0 & 160.3 & 171.3 & 171.3\end{array}$ $\frac{2.74}{1.58} \frac{2.06}{1.28} \frac{2.15}{1.53} \frac{2.47}{1.81} \frac{12.93}{2.37} \frac{2.35}{1.63} \frac{2.22}{1.60} \frac{2.35}{1.74} \frac{2.75}{2.00} \frac{2.28}{1.64} \frac{2.32}{1.70} \frac{2.56}{1.99}$

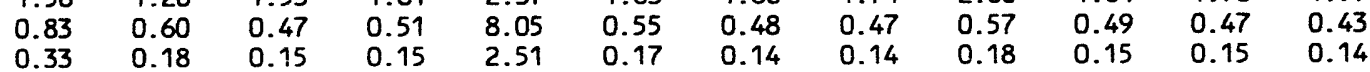

$5,419,251 \quad 52,081,925$
10. \begin{tabular}{cc}
0.0 & 0.0 \\
0.00 & 0.00 \\
\hline 0.00 & 0.00 \\
0.00 & 0.00 \\
0.00 & 0.00
\end{tabular} \begin{tabular}{ll}
.0 & 0.0 \\
00 & 0.00 \\
\hline 00 & 0.00 \\
00 & 0.00 \\
00 & 0.00
\end{tabular} $\begin{array}{r}0.0 \\ 0.00 \\ \hline .00\end{array}$ $\begin{array}{r}0.0 \\ 0.00 \\ \hline 0.00\end{array}$ $0.00 \quad 0.00$ $\begin{array}{lll}0.00 & 0.00 & 0.00\end{array}$ \begin{tabular}{ccccc}
0.0 & 0.0 & 0.0 & 6.2 & 5.6 \\
0.00 & 0.00 & 0.00 & 2.52 & 2.67 \\
\cline { 5 - 5 } & 0.00 & 0.00 & 1.68 & 1.83 \\
0.00 & 0.00 & 0.00 & 0.84 & 0.84 \\
0.00 & 0.00 & 0.00 & 0.00 & 0.00
\end{tabular}

15.

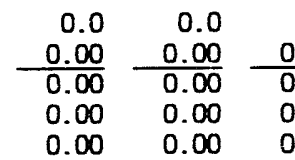
\begin{tabular}{lr}
0.0 & 0.0 \\
.00 & 0.00 \\
\hline 00 & 0.00 \\
00 & 0.00 \\
0.00
\end{tabular}
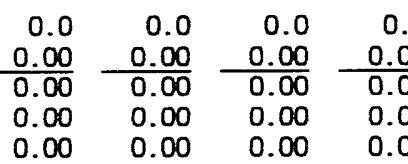

$0.0 \quad 0.0$ \begin{tabular}{rrrr}
0.0 & 0.0 & 9.9 & 11.8 \\
.00 & 0.00 & 2.76 & $\frac{2.79}{2.08}$ \\
\cline { 2 - 4 } & 0.00 & 2.04 & 2.08 \\
.00 & 0.00 & 0.72 & 0.71 \\
.00 & 0.00 & 0.00 & 0.00
\end{tabular}

20.0

\begin{tabular}{|c|c|c|c|c|c|c|c|c|c|c|}
\hline $\begin{array}{r}0.0 \\
0.00 \\
0.00 \\
0.00 \\
0.00\end{array}$ & $\begin{array}{r}0.0 \\
0.00 \\
0.00 \\
0.00 \\
0.00\end{array}$ & $\begin{array}{r}0.0 \\
0.00 \\
0.00 \\
0.00 \\
0.00\end{array}$ & $\begin{array}{r}0.0 \\
0.00 \\
0.00 \\
0.00 \\
0.00\end{array}$ & $\begin{array}{l}0.0 \\
0.00 \\
0.00 \\
0.00 \\
0.00\end{array}$ & $\begin{array}{r}0.0 \\
0.00 \\
0.00 \\
0.00 \\
0.00\end{array}$ & $\begin{array}{l}0.0 \\
0.00 \\
0.00 \\
0.00 \\
0.00\end{array}$ & $\begin{array}{r}0.0 \\
0.00 \\
0.00 \\
0.00 \\
0.00\end{array}$ & $\begin{array}{r}0.0 \\
0.00 \\
0.00 \\
0.00 \\
0.00\end{array}$ & $\begin{array}{l}0.0 \\
0.00 \\
0.00 \\
0.00 \\
0.00\end{array}$ & $\begin{array}{l}12.4 \\
2.39 \\
1.53 \\
0.86 \\
0.00\end{array}$ \\
\hline
\end{tabular}

359,065
663,532

663,532

718,130
359,065 .00 


\section{VOLUME AND PRICE REPORT}

Long Term Imports
Volumes (MMCf/d) \& Prices ( $\$$ MMBTU) of Natural

Gas Imported During the Past 12 Months $01 / 01 / 93-12 / 31 / 93$

\section{Long-Term Importer}

NEW ENGLAND POWER COMPANY

(TRANSWEST ENERGY INC.)

AVG Daily Quantity

Total Price (Waddington, New York) Commodity Component

Reservation Fee component

NEW JERSEY NATURAL GAS

(ALBERTA NORTHEAST GAS (WGM LTD. 1))

AVG Daily Quantity

Total Price (Waddington, New York)

Commodity Component

Demand Component

Reservation fee Component

NEW JERSEY NATURAL GAS

(ALBERTA NORTHEAST GAS (WGM LTD. 2))

AVG Daily Quantity

Total Price (Waddington, New York) Commod ity Component

Reservation fee Component

NEW JERSEY NATURAL GAS

(ALBERTA NORTHEAST GAS (PROGAS))

AVG Daily Quantity

Total Price (Waddington, New York)

Commodity Component

Demand Component

Reservation Fee Component

NEW JERSEY NATURAL GAS

(ALBERTA NORTHEAST GAS (ATCOR))

AVG Daily Quantity

Total Price (Waddington, New York) Comand component

Reservation Fee Component

\begin{tabular}{|c|c|c|c|c|c|c|c|c|c|c|c|c|}
\hline $\begin{array}{l}\text { Auth. } \\
\text { Vols. }\end{array}$ & $\begin{array}{l}1993 \\
\text { Jan. } \\
\end{array}$ & $\begin{array}{l}1993 \\
\text { Feb. }\end{array}$ & $\begin{array}{l}1993 \\
\text { Mar. }\end{array}$ & $\begin{array}{l}1993 \\
\text { Apr. } \\
\end{array}$ & $\begin{array}{l}1993 \\
\text { May }\end{array}$ & $\begin{array}{l}1993 \\
\text { June }\end{array}$ & $\begin{array}{l}1993 \\
\text { July } \\
\end{array}$ & $\begin{array}{l}1993 \\
\text { Aug. }\end{array}$ & $\begin{array}{l}1993 \\
\text { Sep. }\end{array}$ & $\begin{array}{l}1993 \\
\text { Oct. }\end{array}$ & $\begin{array}{l}1993 \\
\text { Nov. }\end{array}$ & $\begin{array}{l}1993 \\
\text { Dec. }\end{array}$ \\
\hline 15.0 & $\begin{array}{r}0.0 \\
0.00 \\
0.00 \\
0.00 \\
0.00\end{array}$ & $\begin{array}{r}0.0 \\
0.00 \\
0.00 \\
0.00 \\
0.00\end{array}$ & $\begin{array}{r}0.0 \\
0.00 \\
0.00 \\
0.00 \\
0.00\end{array}$ & $\begin{array}{l}0.0 \\
0.00 \\
0.00 \\
0.00 \\
0.00\end{array}$ & $\begin{array}{r}0.0 \\
0.00 \\
0.00 \\
0.00 \\
0.00\end{array}$ & $\begin{array}{r}0.0 \\
0.00 \\
0.00 \\
0.00 \\
0.00\end{array}$ & $\begin{array}{l}0.0 \\
0.00 \\
0.00 \\
0.00 \\
0.00\end{array}$ & $\begin{array}{l}0.0 \\
0.00 \\
0.00 \\
0.00 \\
0.00\end{array}$ & $\begin{array}{l}0.0 \\
0.00 \\
0.00 \\
0.00 \\
0.00\end{array}$ & $\begin{array}{r}0.0 \\
0.00 \\
0.00 \\
0.00 \\
0.00\end{array}$ & $\begin{array}{r}9.3 \\
3.05 \\
2.33 \\
0.72 \\
0.00\end{array}$ & $\begin{array}{l}7.4 \\
3.10 \\
2.39 \\
0.71 \\
0.00\end{array}$ \\
\hline 25.2 & $\begin{array}{r}25.1 \\
2.91 \\
1.98 \\
0.93 \\
0.00\end{array}$ & $\begin{array}{l}25.2 \\
2.91 \\
1.96 \\
0.95 \\
0.00\end{array}$ & $\begin{array}{l}25.2 \\
2.91 \\
1.95 \\
0.96 \\
0.00\end{array}$ & $\begin{array}{l}25.2 \\
2.47 \\
1.49 \\
0.98 \\
0.00\end{array}$ & $\begin{array}{l}25.2 \\
2.70 \\
1.73 \\
0.97 \\
0.00\end{array}$ & $\begin{array}{l}25.2 \\
2.37 \\
1.39 \\
0.98 \\
0.00\end{array}$ & $\begin{array}{l}25.2 \\
2.37 \\
1.39 \\
0.98 \\
0.00\end{array}$ & $\begin{array}{l}25.2 \\
2.37 \\
1.40 \\
0.97 \\
0.00\end{array}$ & $\begin{array}{l}25.2 \\
2.37 \\
1.43 \\
0.94 \\
0.00\end{array}$ & $\begin{array}{l}25.2 \\
2.29 \\
1.35 \\
0.94 \\
0.00\end{array}$ & $\begin{array}{l}25.2 \\
2.66 \\
1.73 \\
0.93 \\
0.00\end{array}$ & $\begin{array}{l}25.2 \\
2.69 \\
1.77 \\
0.92 \\
0.00\end{array}$ \\
\hline 5.3 & $\begin{array}{r}5.2 \\
2.91 \\
1.98 \\
0.93 \\
0.00\end{array}$ & $\begin{array}{r}5.3 \\
2.91 \\
1.96 \\
0.95 \\
0.00\end{array}$ & $\begin{array}{r}5.2 \\
2.91 \\
1.95 \\
0.96 \\
0.00\end{array}$ & $\begin{array}{r}5.3 \\
2.47 \\
1.49 \\
0.98 \\
0.00\end{array}$ & $\begin{array}{r}5.3 \\
2.70 \\
1.73 \\
0.97 \\
0.00\end{array}$ & $\begin{array}{r}5.3 \\
2.37 \\
1.39 \\
0.98 \\
0.00\end{array}$ & $\begin{array}{r}5.3 \\
2.37 \\
1.39 \\
0.98 \\
0.00\end{array}$ & $\begin{array}{l}5.3 \\
2.37 \\
1.40 \\
0.97 \\
0.00\end{array}$ & $\begin{array}{r}5.3 \\
2.37 \\
1.43 \\
0.94 \\
0.00\end{array}$ & $\begin{array}{r}5.3 \\
2.29 \\
1.35 \\
0.94 \\
0.00\end{array}$ & $\begin{array}{r}5.3 \\
2.66 \\
1.73 \\
0.93 \\
0.00\end{array}$ & $\begin{array}{r}5.3 \\
2.69 \\
1.77 \\
0.92 \\
0.00\end{array}$ \\
\hline 4.6 & $\begin{array}{r}4.6 \\
2.91 \\
1.97 \\
0.94 \\
0.00\end{array}$ & $\begin{array}{r}4.6 \\
2.91 \\
1.95 \\
0.96 \\
0.00\end{array}$ & $\begin{array}{l}4.6 \\
2.91 \\
1.97 \\
0.94 \\
0.00\end{array}$ & $\begin{array}{r}4.6 \\
2.46 \\
1.48 \\
0.98 \\
0.00\end{array}$ & $\begin{array}{r}4.6 \\
2.76 \\
1.78 \\
0.98 \\
0.00\end{array}$ & $\begin{array}{r}4.6 \\
2.37 \\
1.39 \\
0.98 \\
0.00\end{array}$ & $\begin{array}{l}4.6 \\
2.37 \\
1.38 \\
0.99 \\
0.00\end{array}$ & $\begin{array}{r}4.6 \\
2.37 \\
1.40 \\
0.97 \\
0.00\end{array}$ & $\begin{array}{r}4.6 \\
2.36 \\
1.42 \\
0.94 \\
0.00\end{array}$ & $\begin{array}{r}4.6 \\
2.30 \\
1.35 \\
0.95 \\
0.00\end{array}$ & $\begin{array}{r}4.6 \\
2.67 \\
1.73 \\
0.94 \\
0.00\end{array}$ & $\begin{array}{r}4.6 \\
2.69 \\
1.73 \\
0.96 \\
0.00\end{array}$ \\
\hline 3.0 & $\begin{array}{r}3.0 \\
2.91 \\
2.01 \\
0.90 \\
0.00\end{array}$ & $\begin{array}{r}3.0 \\
2.91 \\
2.01 \\
0.90 \\
0.00\end{array}$ & $\begin{array}{r}3.0 \\
2.92 \\
2.01 \\
0.91 \\
0.00\end{array}$ & $\begin{array}{r}3.0 \\
2.46 \\
1.54 \\
0.92 \\
0.00\end{array}$ & $\begin{array}{l}3.0 \\
2.71 \\
1.78 \\
0.93 \\
0.00\end{array}$ & $\begin{array}{r}3.0 \\
.2 .37 \\
1.45 \\
0.92 \\
0.00\end{array}$ & $\begin{array}{l}3.1 \\
2.37 \\
1.46 \\
0.91 \\
0.00\end{array}$ & $\begin{array}{r}3.0 \\
2.37 \\
1.46 \\
0.91 \\
0.00\end{array}$ & $\begin{array}{r}3.0 \\
2.37 \\
1.48 \\
0.89 \\
0.00\end{array}$ & $\begin{array}{r}3.0 \\
2.30 \\
1.42 \\
0.88 \\
0.00\end{array}$ & $\begin{array}{r}3.0 \\
2.66 \\
1.79 \\
0.87 \\
0.00\end{array}$ & $\begin{array}{r}3.0 \\
2.68 \\
1.80 \\
0.88 \\
0.00\end{array}$ \\
\hline
\end{tabular}

4093 TOTAL MCf TOTAL MCf

$509,730 \quad 509,730$

$2,318,400 \quad 9,218,631$

$483,000 \quad 1,920,564$

$425,040 \quad 1,689,401$

$274,528 \quad 1,094,094$ 


\section{VOLUNE AND PRICE REPORT}

\section{Long Term Imports}

Volumes (MMcf/d) \& Prices ( $\$$ /MMBTU) of Natural

Gas Imported During the Past 12 Months $01 / 01 / 93$ - 12/31/93

\begin{tabular}{|c|c|c|c|c|c|c|c|c|c|c|c|c|c|c|c|}
\hline Long-Term Importer & $\begin{array}{l}\text { Auth. } \\
\text { Vols. }\end{array}$ & $\begin{array}{l}1993 \\
\text { Jan. } \\
\end{array}$ & $\begin{array}{l}1993 \\
\text { Feb. }\end{array}$ & $\begin{array}{l}1993 \\
\text { Mar. }\end{array}$ & $\begin{array}{l}1993 \\
\text { Apr. }\end{array}$ & $\begin{array}{l}1993 \\
\text { May }\end{array}$ & $\begin{array}{r}1993 \\
\text { June }\end{array}$ & $\begin{array}{l}1993 \\
\text { July }\end{array}$ & $\begin{array}{l}1993 \\
\text { Aug. }\end{array}$ & $\begin{array}{l}1993 \\
\text { Sep. }\end{array}$ & $\begin{array}{l}1993 \\
\text { Oct. }\end{array}$ & $\begin{array}{l}1993 \\
\text { Nov. }\end{array}$ & $\begin{array}{l}1993 \\
\text { Dec. }\end{array}$ & $\begin{array}{c}4093 \\
\text { TOTAL MCf }\end{array}$ & $\begin{array}{c}\text { YTD } \\
\text { TOTAL MCf } \\
\end{array}$ \\
\hline $\begin{array}{l}\text { NEW JERSEY NATURAL GAS } \\
\text { (ALBERTA NE GAS (AEC OIL \& GAS COMPANY)) } \\
\text { AVG Daily Quantity } \\
\text { Total Price (Waddington, New York) } \\
\text { Commodity Component } \\
\text { Demand Component } \\
\text { Reservation Fee Component }\end{array}$ & 1.9 & $\begin{array}{r}1.9 \\
2.91 \\
2.02 \\
0.89 \\
0.00\end{array}$ & $\begin{array}{r}1.9 \\
2.92 \\
2.01 \\
0.91 \\
0.00\end{array}$ & $\begin{array}{r}1.9 \\
2.91 \\
2.01 \\
0.90 \\
0.00\end{array}$ & $\begin{array}{r}1.9 \\
2.46 \\
1.53 \\
0.93 \\
0.00\end{array}$ & $\begin{array}{r}1.9 \\
2.71 \\
1.75 \\
0.96 \\
0.00\end{array}$ & $\begin{array}{l}1.9 \\
2.37 \\
1.43 \\
0.94 \\
0.00\end{array}$ & $\begin{array}{r}1.9 \\
2.37 \\
1.45 \\
0.92 \\
0.00\end{array}$ & $\begin{array}{r}1.9 \\
2.37 \\
1.42 \\
0.95 \\
0.00\end{array}$ & $\begin{array}{l}1.9 \\
2.37 \\
1.48 \\
0.89 \\
0.00\end{array}$ & $\begin{array}{r}1.9 \\
2.29 \\
1.47 \\
0.82 \\
0.00\end{array}$ & $\begin{array}{r}1.9 \\
2.66 \\
1.78 \\
0.88 \\
0.00\end{array}$ & $\begin{array}{r}1.9 \\
2.68 \\
1.79 \\
0.89 \\
0.00\end{array}$ & 179,032 & 711,591 \\
\hline $\begin{array}{l}\text { NIAGARA MOHAWK POWER CORPORATION } \\
\text { (WGM LTD.) } \\
\text { AVG Daily Quantity } \\
\text { Total Price (Waddington, New York) } \\
\text { Commodity Component } \\
\text { Demand Component } \\
\text { Reservation Fee Component }\end{array}$ & 51.0 & $\begin{array}{l}50.5 \\
2.26 \\
1.31 \\
0.95 \\
0.00\end{array}$ & $\begin{array}{l}44.9 \\
2.37 \\
1.14 \\
1.23 \\
0.00\end{array}$ & $\begin{array}{l}50.5 \\
2.26 \\
1.27 \\
0.99 \\
0.00\end{array}$ & $\begin{array}{l}51.0 \\
2.09 \\
1.07 \\
1.02 \\
0.00\end{array}$ & $\begin{array}{l}51.0 \\
2.48 \\
1.50 \\
0.98 \\
0.00\end{array}$ & $\begin{array}{l}51.0 \\
2.21 \\
1.18 \\
1.03 \\
0.00\end{array}$ & $\begin{array}{l}51.0 \\
2.01 \\
1.02 \\
0.99 \\
0.00\end{array}$ & $\begin{array}{l}51.0 \\
2.04 \\
1.08 \\
0.96 \\
0.00\end{array}$ & $\begin{array}{l}51.0 \\
2.17 \\
1.20 \\
0.97 \\
0.00\end{array}$ & $\begin{array}{l}51.0 \\
2.06 \\
1.11 \\
0.95 \\
0.00\end{array}$ & $\begin{array}{l}51.1 \\
2.18 \\
1.20 \\
0.98 \\
0.00\end{array}$ & $\begin{array}{l}51.0 \\
2.37 \\
1.45 \\
0.92 \\
0.00\end{array}$ & $4,695,894$ & $18,460,266$ \\
\hline $\begin{array}{l}\text { NORTH JERSEY ENERGY ASSOCIATES } \\
\text { (PROGAS LIMITED) } \\
\text { AVG Daily Quantity } \\
\text { Total Price (Niagara Falls, New York) } \\
\text { Commodity Component } \\
\text { Demand Component } \\
\text { Reservation Fee Component }\end{array}$ & 24.0 & $\begin{array}{l}23.6 \\
2.28 \\
1.45 \\
0.83 \\
0.00\end{array}$ & $\begin{array}{l}22.8 \\
2.42 \\
1.42 \\
1.00 \\
0.00\end{array}$ & $\begin{array}{l}23.6 \\
2.30 \\
1.44 \\
0.86 \\
0.00\end{array}$ & $\begin{array}{l}23.6 \\
2.33 \\
1.42 \\
0.91 \\
0.00\end{array}$ & $\begin{array}{l}23.7 \\
2.30 \\
1.43 \\
0.87 \\
0.00\end{array}$ & $\begin{array}{l}23.6 \\
2.33 \\
1.42 \\
0.91 \\
0.00\end{array}$ & $\begin{array}{l}21.7 \\
2.35 \\
1.38 \\
0.97 \\
0.00\end{array}$ & $\begin{array}{l}21.7 \\
2.35 \\
1.41 \\
0.94 \\
0.00\end{array}$ & $\begin{array}{l}21.7 \\
2.38 \\
1.43 \\
0.95 \\
0.00\end{array}$ & $\begin{array}{l}21.8 \\
2.34 \\
1.42 \\
0.92 \\
0.00\end{array}$ & $\begin{array}{l}21.8 \\
2.37 \\
1.42 \\
0.95 \\
0.00\end{array}$ & $\begin{array}{l}21.7 \\
2.35 \\
1.42 \\
0.93 \\
0.00\end{array}$ & $2,002,960$ & $8,268,912$ \\
\hline $\begin{array}{l}\text { NORTHEAST ENERGY ASSOCIATES } \\
\text { (PROGAS LIMITED) } \\
\text { AVG Daily Quantity } \\
\text { Total Price (Niagara Falls, New York) } \\
\text { Commodity Component } \\
\text { Demand Component } \\
\text { Reservation Fee Component }\end{array}$ & 48.0 & $\begin{array}{l}48.0 \\
2.89 \\
2.02 \\
0.87 \\
0.00\end{array}$ & $\begin{array}{l}48.0 \\
3.03 \\
1.98 \\
1.05 \\
0.00\end{array}$ & $\begin{array}{l}47.9 \\
2.93 \\
1.99 \\
0.94 \\
0.00\end{array}$ & $\begin{array}{l}48.0 \\
2.96 \\
1.97 \\
0.99 \\
0.00\end{array}$ & $\begin{array}{l}48.1 \\
2.94 \\
1.99 \\
0.95 \\
0.00\end{array}$ & $\begin{array}{l}48.0 \\
2.96 \\
1.97 \\
0.99 \\
0.00\end{array}$ & $\begin{array}{l}48.0 \\
2.93 \\
1.97 \\
0.96 \\
0.00\end{array}$ & $\begin{array}{l}48.0 \\
2.93 \\
2.00 \\
0.93 \\
0.00\end{array}$ & $\begin{array}{l}48.0 \\
2.96 \\
2.01 \\
0.95 \\
0.00\end{array}$ & $\begin{array}{r}48.3 \\
2.92 \\
2.01 \\
0.91 \\
0.00\end{array}$ & $\begin{array}{l}48.4 \\
2.95 \\
2.01 \\
0.94 \\
0.00\end{array}$ & $\begin{array}{l}48.1 \\
2.93 \\
2.00 \\
0.93 \\
0.00\end{array}$ & $4,440,635$ & $17,597,263$ \\
\hline $\begin{array}{l}\text { NORTHERN MINNESOTA UTILITIES } \\
\text { (WGM LTD.) } \\
\text { AVG Daily Quantity } \\
\text { Total Price (Noyes, Minnesota) } \\
\text { Commodity Component } \\
\text { Demand Component } \\
\text { Reservation Fee Component }\end{array}$ & 10.0 & $\begin{array}{r}10.0 \\
2.00 \\
1.55 \\
0.45 \\
0.00\end{array}$ & $\begin{array}{r}8.5 \\
2.17 \\
1.55 \\
0.62 \\
0.00\end{array}$ & $\begin{array}{r}6.6 \\
2.26 \\
1.55 \\
0.71 \\
0.00\end{array}$ & $\begin{array}{r}1.5 \\
4.77 \\
1.55 \\
3.22 \\
0.00\end{array}$ & $\begin{array}{r}8.0 \\
2.42 \\
1.83 \\
0.59 \\
0.00\end{array}$ & $\begin{array}{r}6.2 \\
2.66 \\
1.85 \\
0.81 \\
0.00\end{array}$ & $\begin{array}{l}5.5 \\
2.73 \\
1.85 \\
0.88 \\
0.00\end{array}$ & $\begin{array}{l}10.0 \\
2.31 \\
1.85 \\
0.46 \\
0.00\end{array}$ & $\begin{array}{l}10.0 \\
2.31 \\
1.85 \\
0.46 \\
0.00\end{array}$ & $\begin{array}{r}8.7 \\
2.38 \\
1.85 \\
0.53 \\
0.00\end{array}$ & $\begin{array}{r}8.4 \\
2.41 \\
1.85 \\
0.56 \\
0.00\end{array}$ & $\begin{array}{r}7.9 \\
2.39 \\
1.85 \\
0.54 \\
0.00\end{array}$ & 767,071 & $2,792,673$ \\
\hline
\end{tabular}




\section{VOLUNE AND PRICE REPORT}

Long Term Imports

Volumes (MMcf/d) \& Prices ( $\$ / M M B T U$ ) of Natural

Gas Imported During the Past 12 Months $01 / 01 / 93-12 / 31 / 93$

\section{Long-Term Importer}

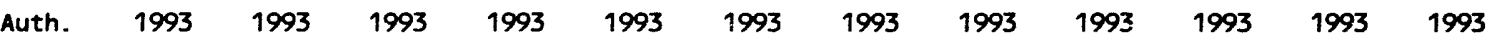

Vols. Jan. Feb. Mar. Apr. May June July Aug. Sep. oct. Nov. Dec.

4093

YTD

NORTHERN MINNESOTA UTILITIES - EASTERN MARKET (1)

(WGM LTD.)

AVG Daily Quantity

$29.8 \quad 21$.

Total Price (International Falls, MN)

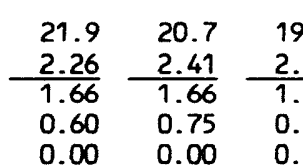

\begin{tabular}{llll}
18.1 & 14.9 & 15.2 \\
& 2.45 & 2.57 & 2.60 \\
\hline 1.65 & 1.65 & 1.65 \\
\hline 0.80 & 0.92 & 0.95 \\
0.00 & 0.00 & 0.00
\end{tabular}

\begin{tabular}{lllllll}
14.9 & 13.4 & 14.6 & 17.3 & 19.7 & 20.1 \\
2.60 & 2.65 & 2.57 & 2.43 & 2.77 & 2.70 \\
\hline 1.65 & 1.65 & 1.65 & 1.65 & 2.07 & 2.07 \\
0.95 & 1.00 & 0.92 & 0.78 & 0.70 & 0.63 \\
0.00 & 0.00 & 0.00 & 0.00 & 0.00 & 0.00
\end{tabular}

Reservation Fee Component

NORTHERN MINNESOTA UTILITIES - WESTERN MARKET (2)

(WGM LTD.)

AVG Daily Quantity

1.5

Total Price (Warroad, Minnesota)

Commodity Component

Demand Component
Reservation Fee Component

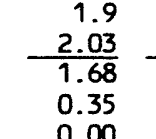

$\begin{array}{r}1.9 \\ 2.09 \\ \hline .68\end{array}$

$\begin{array}{llllll}1.4 & 0.8 & 0.5 & 0.3 & 0.3 & 0.3\end{array}$

$\begin{array}{llllll}0.00 & 0.00 & 0.51 & 0.95 & 1.43 & 2.22 \\ & 0.00 & 0.00 & 0.00 & 0.00\end{array}$

\begin{tabular}{ll}
0.11 & 3.79 \\
\hline 1.68 & $\frac{1.68}{1.68}$ \\
2.43 & 2.11 \\
0.00 & 0.00
\end{tabular}

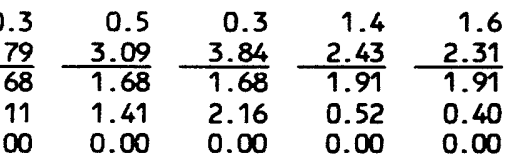

99,617

337,748

(WGM LTD.)

AVG Daily Quantity

Total Price (Warroad, Minnesota)

Commodity Component

Demand Component

Reservation Fee Component

NORTHERN NATURAL GAS COMPANY (3)

(WESTCOAST GAS SERVICES)

AVG Daily Quantity

Total Price (Port of Morgan, Montana)

100.0

\begin{tabular}{|c|c|c|c|c|c|c|c|c|c|c|c|}
\hline $\begin{array}{l}68.4 \\
2.00 \\
1.77 \\
0.23 \\
0.00\end{array}$ & $\begin{array}{l}71.7 \\
1.80 \\
1.46 \\
0.34 \\
0.00\end{array}$ & $\begin{array}{r}100.1 \\
1.87 \\
1.67 \\
0.20 \\
0.00\end{array}$ & $\begin{array}{l}50.4 \\
2.19 \\
1.90 \\
0.29 \\
0.00\end{array}$ & $\begin{array}{l}30.0 \\
2.82 \\
2.36 \\
0.46 \\
0.00\end{array}$ & $\begin{array}{l}60.2 \\
1.93 \\
1.69 \\
0.24 \\
0.00\end{array}$ & $\begin{array}{l}60.3 \\
\frac{1.87}{1.64} \\
0.23 \\
0.00\end{array}$ & $\begin{array}{l}54.4 \\
2.00 \\
1.75 \\
0.25 \\
0.00\end{array}$ & $\begin{array}{l}52.7 \\
2.23 \\
1.99 \\
0.24 \\
0.00\end{array}$ & $\begin{array}{l}49.1 \\
1.97 \\
1.72 \\
0.25 \\
0.00\end{array}$ & $\begin{array}{r}0.0 \\
0.00 \\
0.00 \\
0.00 \\
0.00\end{array}$ & $\begin{array}{r}0.0 \\
0.00 \\
0.00 \\
0.00 \\
0.00\end{array}$ \\
\hline
\end{tabular}

(1) This import contract was previously shown as imports made by Inter-City Minnesota Pipelines, Ltd. - 1 .

(2) This import contract was previously shown as imports made by Inter-City Minnesota Pipel ines, Ltd. - 2 .
(3) Pursuant to DOE/FE Order 874 , issued October 29, 1993, this authorization was terminated and the supplies were assigned to other parties. 


\section{VOLUNE AND PRICE REPORT \\ Iong Term Imports}

Volumes (MMcf/d) \& Prices ( $\$$ /MMBTU) of Natural

Gas Imported During the Past 12 Months $01 / 01 / 93$ - 12/31/93

\section{Long-Term Importer}

NORTHERN NATURAL GAS COMPANY (1)

(WGM LTD.)

AVG Daily Quantity

Total Price (Noyes, Minnesota)

Commodity Component

Demand Component

Reservation Fee Component

NORTHERN NATURAL GAS COMPANY (2)

(MOBIL GAS CANADA)

AVG Daily Quantity

Demand Component

Reservation Fee Component

NORTHERN STATES POWER COMPANY

(CANADIAN OCCIDENTAL PETROLEUM COMPANY LTD.)

AVG Daily Quantity

Total Price (Noyes, Minnesota)

Commodity Component

Demand Component

Reservation Fee Component

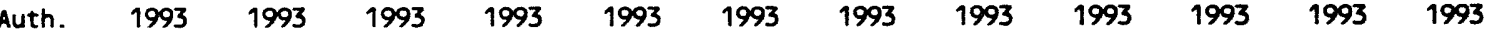

Vols. Jan. Feb. Mar. Apr. May June July Aug. Sep. oct. Nov. Dec.

47.

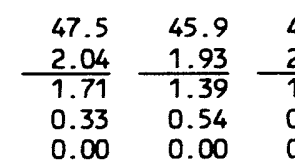

\begin{tabular}{lll}
47.5 & 43.4 & 31.9 \\
2.08 & 2.36 & 2.99 \\
\cline { 3 - 3 } 1.61 & 1.83 & 2.30 \\
0.47 & 0.53 & 0.69 \\
0.00 & 0.00 & 0.00
\end{tabular}

\begin{tabular}{ll}
9 & 47 \\
90 & 2.1 \\
\hline 69 & 1.62 \\
00 & 0.50
\end{tabular}

20.0

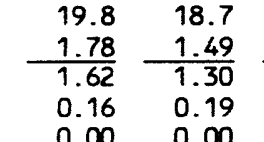

\begin{tabular}{lr}
20.1 & 11.6 \\
1.68 & 2.09 \\
\hline 1.52 & 1.81 \\
0.16 & 0.28 \\
0.00 & 0.00
\end{tabular}

\begin{tabular}{r}
9.4 \\
2.60 \\
\hline 2.27 \\
0.33 \\
0.00
\end{tabular}

12.1
$\frac{1.85}{1.58}$
0.27
0.00

\begin{tabular}{l}
11.9 \\
1.79 \\
\hline 1.53 \\
0.26 \\
0.00
\end{tabular}

7.5

\begin{tabular}{rrrrr}
7.5 & 7.5 & 7.5 & 7.5 & 7.5 \\
2.33 & 2.31 & 2.09 & 1.99 & 1.96 \\
\hline 1.91 & 1.84 & 1.66 & 1.55 & 1.54 \\
0.42 & 0.47 & 0.43 & 0.44 & 0.42 \\
0.00 & 0.00 & 0.00 & 0.00 & 0.00
\end{tabular}

7.5
$\frac{1.91}{1.48}$
0.43

$\begin{array}{rr}7.5 & 7.1 \\ 1.92 & 1.97 \\ & 1.51 \\ 0.41 & 0.43 \\ 0.00 & 0.00\end{array}$

\begin{tabular}{rrrrr}
7.1 & 7.5 & 7.5 & 7.5 & 7.5 \\
1.97 & 1.91 & 2.15 & 2.14 & 2.43 \\
\hline 1.54 & 1.55 & 1.75 & 1.71 & 2.00 \\
0.43 & 0.36 & 0.40 & 0.43 & 0.43 \\
0.00 & 0.00 & 0.00 & 0.00 & 0.00
\end{tabular}

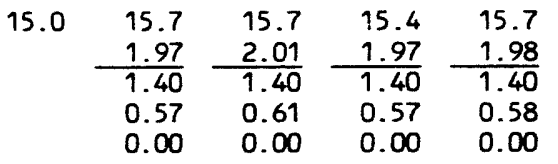

15.7
1.96
1.40
0.56
0.00

\begin{tabular}{r}
15.6 \\
1.97 \\
\hline 1.40 \\
0.57
\end{tabular}

15.6

$14.7 \quad 15.6$

AMG Daily Quant ity

Total Frice (Noyes, Minnesota)

Reservation Fee Component

NORTHERN STATES POWER COMPANY

(PROGAS LIMITED)

AVG Daily Quantity

Total Price (Noyes, Minnesota)

Commodity Component

Demand Component

Reservation fee Component \begin{tabular}{rrrrrrrrrrrrrr}
7.5 & 7.5 & 7.5 & 7.2 & 6.4 & 6.3 & 7.5 & 7.4 & 7.3 & 7.4 & 7.0 & 7.7 & 7.4 \\
& 2.42 & 2.47 & 2.08 & 2.09 & 2.06 & -1.87 & 1.96 & 1.93 & 2.02 & 2.13 & 2.16 & $\frac{2.31}{1.31}$ \\
\hline 1.96 & 1.96 & 1.59 & 1.53 & $\frac{1.51}{1.40}$ & 1.50 & 1.47 & 1.56 & 1.66 & 1.71 & 1.85 \\
0.46 & 0.51 & 0.49 & 0.56 & 0.55 & 0.47 & 0.46 & 0.46 & 0.46 & 0.47 & 0.45 & 0.46 \\
0.00 & 0.00 & 0.00 & 0.00 & 0.00 & 0.00 & 0.00 & 0.00 & 0.00 & 0.00 & 0.00 & 0.00
\end{tabular}
689,505

$2,730,051$

4093 YTD

TOTAL MCf TOTAL MCf

$1,267,500 \quad 12,591,228$

438,452 5,685,584

$678,296 \quad 2,641,723$
$309,650 \quad 4,036,939$

(1) Pursuant to DOE/FE Order 873, issued October 29, 1993, this authorization was terminated and the supplies were assigned to other parties.

(2) On November 3, 1993, DOE/FE Opinion and Order Number 528-A terminated this authorization. 
VOLUME AND PRICE REPORT
LOng Term Imports

Volumes (MMCf/d)

Gas Imported During the Past 12 Months $01 / 01 / 93^{-} 12 / 31 / 93$

Long-Term Importer

NORTHWEST NATURAL GAS COMPANY

(CANWEST GAS SUPPLY)

AVG Daily Quantity

Total Price (Sumas, Washington) Commodi ty Component

Reservation fee component

NORTHWEST NATURAL GAS COMPANY

(AMOCO CANADA PETROLEUM CO., LTD.)

AVG Daily Quantity

Total Price (Sumas, Washington)

Commodity Component

Demand Component

Reservation Fee Component

Auth. 1993

$92.0 \quad 86$

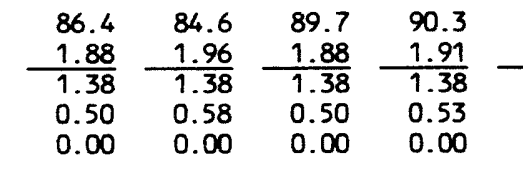

$\begin{array}{lllll}1993 & 1993 & 1993 & 1993 & 1993 \\ \text { Dec. }\end{array}$

4093

TOTAL MCf TOTAL MCf

NORTHWEST NATURAL GAS COMPANY

(POCO PETROLEUMS LIMITED)

AVG Daily Quantity

Total Price (Eastport, Idaho)

Commodity Compone

Reservation Fee Component

NORTHWEST NATURAL GAS COMPANY

(SUMMIT RESOURCES LIMITED)

AVG Daily Quantity

Total Price (Eastport, Idaho)

Commodity Component

Demand Component

Reservation Fee Component

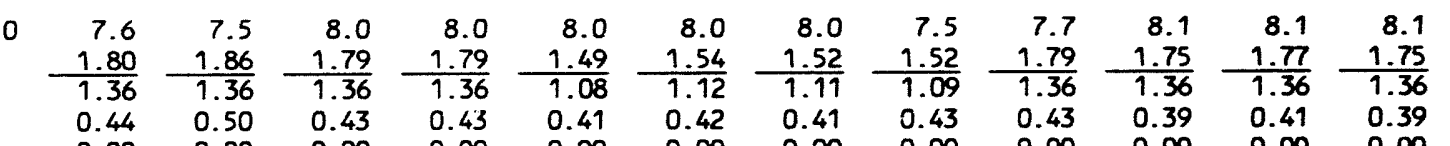

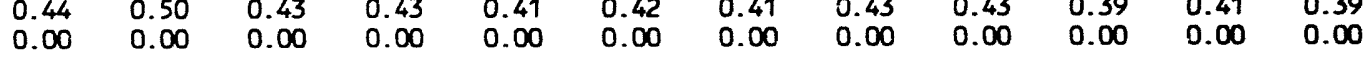

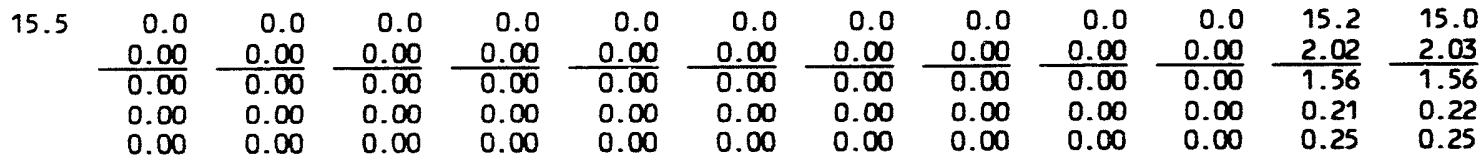

919,826

8.0

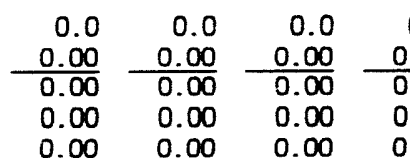

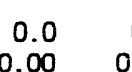

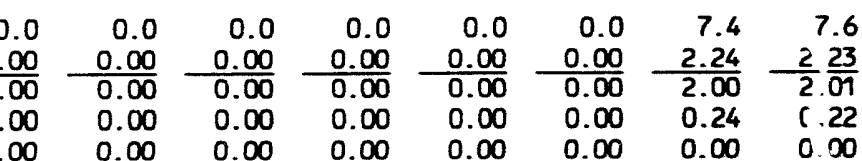

455,695

455,695

NORTHWEST NATURAL GAS COMPANY

(WESTCOAST GAS SERVICES)

AVG Daily Quantity

Total Price (Eastport, Idaho)

Commodity Component

Reservation Fee Component

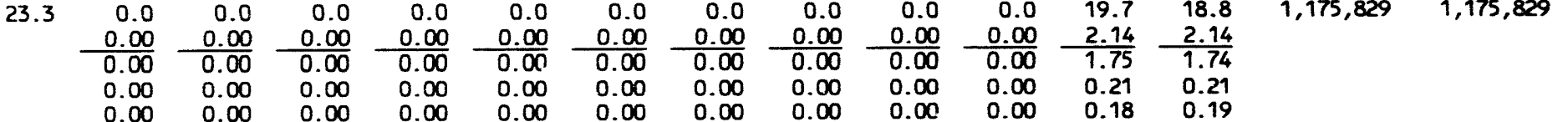




\section{VOLURE AND PRICE REPORT}

Long Term Imports

Lolumes (MMcf/d) \& Prices (S/MMBTU) of Natural

Volumes (MMcf/d) \& Prices (\$/MMBTO) of Natural
Gas Imported During the Past 12 Months $01 / 01 / 93-12 / 31 / 93$

\begin{tabular}{|c|c|c|c|c|c|c|c|c|c|c|c|c|c|c|c|}
\hline Long-Term Importer & $\begin{array}{l}\text { Auth. } \\
\text { Vois. }\end{array}$ & $\begin{array}{l}1993 \\
\text { Jan. } \\
\end{array}$ & $\begin{array}{l}1993 \\
\text { feb. }\end{array}$ & $\begin{array}{l}1993 \\
\text { Mar. }\end{array}$ & $\begin{array}{l}1993 \\
\text { Apr. }\end{array}$ & $\begin{array}{l}1993 \\
\text { May } \\
\end{array}$ & $\begin{array}{l}1993 \\
\text { June } \\
\end{array}$ & $\begin{array}{l}1993 \\
\text { July } \\
\end{array}$ & $\begin{array}{l}1993 \\
\text { Aug. }\end{array}$ & $\begin{array}{l}1993 \\
\text { Sep. }\end{array}$ & $\begin{array}{l}1993 \\
\text { Oct. }\end{array}$ & $\begin{array}{l}1993 \\
\text { Nov. }\end{array}$ & $\begin{array}{l}1993 \\
\text { Dec. }\end{array}$ & $\begin{array}{c}4093 \\
\text { TOTAL Mcf }\end{array}$ & $\begin{array}{c}\text { YTD } \\
\text { TOTAL MCf }\end{array}$ \\
\hline $\begin{array}{l}\text { NORTHWEST NATURAL GAS COMPANY } \\
\text { (CANADIAN HYDROCARBONS MARKETING) } \\
\text { AVG Daily Quantity } \\
\text { Total Price (Eastport, Idaho) } \\
\text { Commodity Component } \\
\text { Demand Component } \\
\text { Reservation Fee Component }\end{array}$ & 3.8 & $\begin{array}{l}0.0 \\
0.00 \\
0.00 \\
0.00 \\
0.00\end{array}$ & $\begin{array}{l}0.0 \\
0.00 \\
0.00 \\
0.00 \\
0.00\end{array}$ & $\begin{array}{r}0.0 \\
0.00 \\
0.00 \\
0.00 \\
0.00\end{array}$ & $\begin{array}{r}0.0 \\
0.00 \\
0.00 \\
0.00 \\
0.00\end{array}$ & $\begin{array}{r}0.0 \\
0.00 \\
0.00 \\
0.00 \\
0.00\end{array}$ & $\begin{array}{r}0.0 \\
0.00 \\
0.00 \\
0.00 \\
0.00\end{array}$ & $\begin{array}{l}0.0 \\
0.00 \\
0.00 \\
0.00 \\
0.00\end{array}$ & $\begin{array}{l}0.0 \\
0.00 \\
0.00 \\
0.00 \\
0.00\end{array}$ & $\begin{array}{r}0.0 \\
0.00 \\
0.00 \\
0.00 \\
0.00\end{array}$ & $\begin{array}{r}0.0 \\
0.00 \\
0.00 \\
0.00 \\
0.00\end{array}$ & $\begin{array}{r}0.7 \\
2.97 \\
1.82 \\
1.15 \\
0.00\end{array}$ & $\begin{array}{r}0.9 \\
3.58 \\
2.41 \\
1.17 \\
0.00\end{array}$ & 48,527 & 48,527 \\
\hline $\begin{array}{l}\text { NORTHWEST PIPELINE CORPORATION (1) } \\
\text { (CANADIAN HYDROCARBONS MARKETING) } \\
\text { AVG Daily Quantity } \\
\text { Total Price (Eastport, Idaho) } \\
\text { Commodity Component } \\
\text { Demand Component } \\
\text { Reservation Fee Component }\end{array}$ & 151.7 & $\begin{array}{r}119.1 \\
1.63 \\
1.43 \\
0.20 \\
0.00\end{array}$ & $\begin{array}{l}97.5 \\
1.69 \\
1.43 \\
0.26 \\
0.00\end{array}$ & $\begin{array}{l}32.3 \\
2.23 \\
1.52 \\
0.71 \\
0.00\end{array}$ & $\begin{array}{l}78.0 \\
1.68 \\
1.30 \\
0.38 \\
0.00\end{array}$ & $\begin{array}{l}91.8 \\
1.66 \\
1.31 \\
0.35 \\
0.00\end{array}$ & $\begin{array}{l}18.6 \\
3.02 \\
1.30 \\
1.72 \\
0.00\end{array}$ & $\begin{array}{r}17.2 \\
2.99 \\
1.32 \\
1.67 \\
0.00\end{array}$ & $\begin{array}{l}70.9 \\
1.77 \\
1.35 \\
0.42 \\
0.00\end{array}$ & $\begin{array}{l}87.7 \\
1.66 \\
1.37 \\
0.29 \\
0.00\end{array}$ & $\begin{array}{l}50.5 \\
1.83 \\
1.24 \\
0.59 \\
0.00\end{array}$ & $\begin{array}{r}0.0 \\
0.00 \\
0.00 \\
0.00 \\
0.00\end{array}$ & $\begin{array}{l}0.0 \\
0.00 \\
0.00 \\
0.00 \\
0.00\end{array}$ & $1,566,539$ & $20,198,602$ \\
\hline $\begin{array}{l}\text { NW ALASKAN PIPELINE CO-NORTHERN NATURAL } \\
\text { (PAN-ALBERTA GAS LTD.) } \\
\text { AVG Daily Quantity } \\
\text { Total Price (Port of Morgan, Montana) } \\
\text { Commodity Component } \\
\text { Demand Component } \\
\text { Reservation Fee Component }\end{array}$ & 200.0 & $\begin{array}{r}123.9 \\
2.16 \\
1.67 \\
0.49 \\
0.00\end{array}$ & $\begin{array}{r}140.8 \\
1.84 \\
1.36 \\
0.48 \\
0.00\end{array}$ & $\begin{array}{r}199.2 \\
1.87 \\
1.56 \\
0.31 \\
0.00\end{array}$ & $\begin{array}{r}179.0 \\
2.13 \\
1.78 \\
0.35 \\
0.00\end{array}$ & $\begin{array}{r}131.6 \\
2.66 \\
2.20 \\
0.46 \\
0.00\end{array}$ & $\begin{array}{r}194.8 \\
1.85 \\
1.53 \\
0.32 \\
0.00\end{array}$ & $\begin{array}{r}194.5 \\
1.72 \\
1.51 \\
0.21 \\
0.00\end{array}$ & $\begin{array}{r}192.9 \\
1.74 \\
1.53 \\
0.21 \\
0.00\end{array}$ & $\begin{array}{r}193.6 \\
2.00 \\
1.79 \\
0.21 \\
0.00\end{array}$ & $\begin{array}{r}146.4 \\
1.93 \\
1.66 \\
0.27 \\
0.00\end{array}$ & $\begin{array}{r}199.2 \\
1.81 \\
1.60 \\
0.21 \\
0.00\end{array}$ & $\begin{array}{r}198.6 \\
2.17 \\
1.97 \\
0.20 \\
0.00\end{array}$ & $16,672,418$ & $63,879,189$ \\
\hline $\begin{array}{l}\text { NW ALASKAN PIPELINE CO-PACIFIC INTERSTATE } \\
\text { (PAN-ALBERTA GAS LTD.) } \\
\text { AVG Daily Quantity } \\
\text { Total Price (Eastport, Idaho) } \\
\text { Commodity Component } \\
\text { Demand Component } \\
\text { Reservation fee Component }\end{array}$ & 240.0 & $\begin{array}{r}240.0 \\
1.97 \\
1.53 \\
0.44 \\
0.00\end{array}$ & $\begin{array}{r}239.0 \\
1.95 \\
1.46 \\
0.49 \\
0.00\end{array}$ & $\begin{array}{r}240.1 \\
1.96 \\
1.52 \\
0.44 \\
0.00\end{array}$ & $\begin{array}{r}237.2 \\
1.87 \\
1.43 \\
0.44 \\
0.00\end{array}$ & $\begin{array}{r}236.2 \\
2.20 \\
1.71 \\
0.49 \\
0.00\end{array}$ & $\begin{array}{r}226.3 \\
1.87 \\
1.43 \\
0.44 \\
0.00\end{array}$ & $\begin{array}{r}214.2 \\
1.94 \\
1.56 \\
0.38 \\
0.00\end{array}$ & $\begin{array}{r}192.6 \\
2.00 \\
1.58 \\
0.42 \\
0.00\end{array}$ & $\begin{array}{r}217.6 \\
1.96 \\
1.57 \\
0.39 \\
0.00\end{array}$ & $\begin{array}{r}231.9 \\
1.92 \\
1.57 \\
0.35 \\
0.00\end{array}$ & $\begin{array}{r}236.0 \\
1.99 \\
1.64 \\
0.35 \\
0.00\end{array}$ & $\begin{array}{r}234.6 \\
1.99 \\
1.65 \\
0.34 \\
0.00\end{array}$ & $21,542,789$ & $83,721,276$ \\
\hline $\begin{array}{l}\text { NW ALASKAN PIPELINE CO-PAN-ALBERTA GAS US } \\
\text { (PAN-ALBERTA GAS LTD.) } \\
\text { AVG Daily Quantity } \\
\text { Total Price (Port of Morgan, Montana) } \\
\text { Commodity Component } \\
\text { Demand Component } \\
\text { Reservation Fee Component }\end{array}$ & 600.0 & $\begin{array}{r}610.9 \\
1.57 \\
1.57 \\
0.00 \\
0.00\end{array}$ & $\begin{array}{r}621.0 \\
1.25 \\
1.25 \\
0.00 \\
0.00\end{array}$ & $\begin{array}{r}588.6 \\
1.58 \\
1.58 \\
0.00 \\
0.00\end{array}$ & $\begin{array}{r}536.3 \\
1.72 \\
1.72 \\
0.00 \\
0.00\end{array}$ & $\begin{array}{r}375.0 \\
1.78 \\
1.78 \\
0.00 \\
0.00\end{array}$ & $\begin{array}{r}571.5 \\
1.40 \\
1.40 \\
0.00 \\
0.00\end{array}$ & $\begin{array}{r}549.4 \\
1.41 \\
1.41 \\
0.00 \\
0.00\end{array}$ & $\begin{array}{r}552.3 \\
1.54 \\
1.54 \\
0.00 \\
0.00\end{array}$ & $\begin{array}{r}546.8 \\
1.80 \\
1.80 \\
0.00 \\
0.00\end{array}$ & $\begin{array}{r}551.8 \\
1.46 \\
1.46 \\
0.00 \\
0.00\end{array}$ & $\begin{array}{r}505.7 \\
1.47 \\
1.47 \\
0.00 \\
0.00\end{array}$ & $\begin{array}{r}562.5 \\
1.81 \\
1.81 \\
0.00 \\
0.00\end{array}$ & $49,714,179$ & $200,324,433$ \\
\hline
\end{tabular}

(1) Contract assigned to other parties, effective November 1, 1993. 


\section{VOLUNE AND PRICE REPORT}

Long Term Imports

Volumes (MMCf/d) \& Prices (S/MMBTU) of Natural

Gas Imported During the Past 12 Months $01 / 01 / 93$ - 12/31/93

\begin{tabular}{|c|c|c|c|c|c|c|c|c|c|c|c|c|c|c|c|}
\hline Long-Term Importer & $\begin{array}{l}\text { Auth. } \\
\text { vols. }\end{array}$ & $\begin{array}{l}1993 \\
\text { Jan. }\end{array}$ & $\begin{array}{l}1993 \\
\text { Feb. }\end{array}$ & $\begin{array}{l}1993 \\
\text { Mar. }\end{array}$ & $\begin{array}{l}1993 \\
\text { Apr. }\end{array}$ & $\begin{array}{l}1993 \\
\text { May } \\
\end{array}$ & $\begin{array}{l}1993 \\
\text { June } \\
\end{array}$ & $\begin{array}{l}1993 \\
\text { July } \\
\end{array}$ & $\begin{array}{l}1993 \\
\text { Aug. }\end{array}$ & $\begin{array}{l}1993 \\
\text { Sep. }\end{array}$ & $\begin{array}{l}1993 \\
\text { Oct. }\end{array}$ & $\begin{array}{l}1993 \\
\text { Nov. }\end{array}$ & $\begin{array}{l}1993 \\
\text { Dec. }\end{array}$ & $\begin{array}{c}4093 \\
\text { TOTAL Mcf }\end{array}$ & $\begin{array}{c}\text { YTo } \\
\text { roTAL nef }\end{array}$ \\
\hline $\begin{array}{l}\text { OCEAN STATE POWER } \\
\text { (PROGAS LIMITED) } \\
\text { AVG Daily Quantity } \\
\text { Total Price (Niagara Falls, New York) } \\
\text { Commodity Component } \\
\text { Demand Component } \\
\text { Reservation Fee Component }\end{array}$ & 50.0 & $\begin{array}{l}50.0 \\
1.96 \\
1.02 \\
0.94 \\
0.00\end{array}$ & $\begin{array}{l}50.0 \\
1.97 \\
1.03 \\
0.94 \\
0.00\end{array}$ & $\begin{array}{l}50.0 \\
1.97 \\
1.05 \\
0.92 \\
0.00\end{array}$ & $\begin{array}{l}50.0 \\
1.99 \\
1.02 \\
0.97 \\
0.00\end{array}$ & $\begin{array}{l}50.0 \\
2.02 \\
1.06 \\
0.96 \\
0.00\end{array}$ & $\begin{array}{l}50.0 \\
2.04 \\
1.08 \\
0.96 \\
0.00\end{array}$ & $\begin{array}{l}50.8 \\
2.05 \\
1.10 \\
0.95 \\
0.00\end{array}$ & $\begin{array}{l}50.2 \\
2.05 \\
1.09 \\
0.96 \\
0.00\end{array}$ & $\begin{array}{l}51.2 \\
2.07 \\
1.10 \\
0.97 \\
0.00\end{array}$ & $\begin{array}{l}50.0 \\
2.06 \\
1.12 \\
0.94 \\
0.00\end{array}$ & $\begin{array}{l}50.0 \\
2.05 \\
1.13 \\
0.92 \\
0.00\end{array}$ & $\begin{array}{l}50.0 \\
2.03 \\
1.10 \\
0.93 \\
0.00\end{array}$ & $4,600,000$ & $18,364,909$ \\
\hline $\begin{array}{l}\text { OCEAN STATE POWER II } \\
\text { (PROGAS LIMITED) } \\
\text { AVG Daily Quantity } \\
\text { Total Price (Niagara Falls, New York) } \\
\text { Commodity Component } \\
\text { Demand Component } \\
\text { Reservation Fee Component }\end{array}$ & 25.0 & $\begin{array}{l}25.0 \\
1.96 \\
1.02 \\
0.94 \\
0.00\end{array}$ & $\begin{array}{l}25.0 \\
1.97 \\
1.03 \\
0.94 \\
0.00\end{array}$ & $\begin{array}{l}25.0 \\
1.97 \\
1.05 \\
0.92 \\
0.00\end{array}$ & $\begin{array}{l}25.0 \\
2.00 \\
1.03 \\
0.97 \\
0.00\end{array}$ & $\begin{array}{l}25.0 \\
2.02 \\
1.06 \\
0.96 \\
0.00\end{array}$ & $\begin{array}{l}25.0 \\
2.02 \\
1.08 \\
0.94 \\
0.00\end{array}$ & $\begin{array}{l}25.4 \\
2.05 \\
1.10 \\
0.95 \\
0.00\end{array}$ & $\begin{array}{l}25.4 \\
2.05 \\
1.09 \\
0.96 \\
0.00\end{array}$ & $\begin{array}{l}25.1 \\
2.07 \\
1.10 \\
0.97 \\
0.00\end{array}$ & $\begin{array}{l}25.0 \\
2.06 \\
1.12 \\
0.94 \\
0.00\end{array}$ & $\begin{array}{l}25.0 \\
2.06 \\
1.12 \\
0.94 \\
0.00\end{array}$ & $\begin{array}{l}25.0 \\
2.03 \\
1.10 \\
0.93 \\
0.00\end{array}$ & $2,300,000$ & $9,17 \pi, 740$ \\
\hline $\begin{array}{l}\text { OCEAN STATE POWER II } \\
\text { (WGM LTD.) } \\
\text { AVG Daily Quantity } \\
\text { Total Price (Niagara Falls, New York) } \\
\text { Comadity Component } \\
\text { Demand Component } \\
\text { Reservation Fee Component }\end{array}$ & 25.0 & $\begin{array}{l}25.0 \\
1.97 \\
1.03 \\
0.94 \\
0.00\end{array}$ & $\begin{array}{l}25.0 \\
1.97 \\
1.03 \\
0.94 \\
0.00\end{array}$ & $\begin{array}{l}25.0 \\
1.97 \\
1.06 \\
0.91 \\
0.00\end{array}$ & $\begin{array}{l}25.0 \\
1.99 \\
1.03 \\
0.96 \\
0.00\end{array}$ & $\begin{array}{l}25.0 \\
2.02 \\
1.06 \\
0.96 \\
0.00\end{array}$ & $\begin{array}{l}25.0 \\
2.04 \\
1.08 \\
0.96 \\
0.00\end{array}$ & $\begin{array}{l}25.4 \\
2.06 \\
1.11 \\
0.95 \\
0.00\end{array}$ & $\begin{array}{l}25.4 \\
2.05 \\
1.09 \\
0.96 \\
0.00\end{array}$ & $\begin{array}{l}25.4 \\
2.07 \\
1.10 \\
0.97 \\
0.00\end{array}$ & $\begin{array}{l}25.0 \\
2.06 \\
1.13 \\
0.93 \\
0.00\end{array}$ & $\begin{array}{l}25.0 \\
2.06 \\
1.13 \\
0.93 \\
0.00\end{array}$ & $\begin{array}{l}25.0 \\
2.03 \\
1.11 \\
0.92 \\
0.00\end{array}$ & $2,300,000$ & $9,187,550$ \\
\hline $\begin{array}{l}\text { ORCHARD GAS CORPORATION (1) } \\
\text { (PROGAS LIAITED) } \\
\text { AVG Daily Quantity } \\
\text { TOtal Price (Waddington, New York) } \\
\text { Commodity Component } \\
\text { Demand Component } \\
\text { Reservation Fee Component }\end{array}$ & 25.0 & $\begin{array}{l}24.8 \\
2.63 \\
1.63 \\
1.00 \\
0.00\end{array}$ & $\begin{array}{l}25.0 \\
2.63 \\
1.63 \\
1.00 \\
0.00\end{array}$ & $\begin{array}{l}25.0 \\
2.63 \\
1.63 \\
1.00 \\
0.00\end{array}$ & $\begin{array}{l}24.9 \\
2.15 \\
1.15 \\
1.00 \\
0.00\end{array}$ & $\begin{array}{l}25.0 \\
2.15 \\
1.15 \\
1.00 \\
0.00\end{array}$ & $\begin{array}{l}24.9 \\
2.15 \\
1.15 \\
1.00 \\
0.00\end{array}$ & $\begin{array}{l}25.0 \\
2.15 \\
1.15 \\
1.00 \\
0.00\end{array}$ & $\begin{array}{l}25.0 \\
\frac{3.03}{2.05} \\
0.98 \\
0.00\end{array}$ & $\begin{array}{l}24.9 \\
2.98 \\
2.00 \\
0.98 \\
0.00\end{array}$ & $\begin{array}{l}24.6 \\
1.92 \\
0.95 \\
0.97 \\
0.00\end{array}$ & $\begin{array}{l}25.2 \\
3.04 \\
2.07 \\
0.97 \\
0.00\end{array}$ & $\begin{array}{l}16.9 \\
2.95 \\
1.97 \\
0.98 \\
0.00\end{array}$ & $2,041,673$ & $8,879,020$ \\
\hline $\begin{array}{l}\text { PACIFIC GAS TRANSMISSION COMPANY (2) } \\
\text { (ALBERTA \& SOUTHERN GAS COMPANY) } \\
\text { AVG Daily Quantity } \\
\text { Total Price (Eastport, Idaho) } \\
\text { Commodity Component } \\
\text { Demand Component } \\
\text { Reservation Fee Component } \\
\text { Calif. Border Price }\end{array}$ & 1023.0 & $\begin{array}{r}1061.3 \\
1.99 \\
1.65 \\
0.34 \\
0.00 \\
2.22\end{array}$ & $\begin{array}{r}1069.2 \\
2.00 \\
1.62 \\
0.38 \\
0.00 \\
2.23\end{array}$ & $\begin{array}{r}809.6 \\
2.05 \\
1.60 \\
0.45 \\
0.00 \\
2.28\end{array}$ & $\begin{array}{r}973.5 \\
2.11 \\
1.69 \\
0.42 \\
0.00 \\
2.31\end{array}$ & $\begin{array}{r}763.9 \\
2.07 \\
1.62 \\
0.45 \\
0.00 \\
2.27\end{array}$ & $\begin{array}{r}883.3 \\
1.79 \\
1.55 \\
0.24 \\
0.00 \\
1.99\end{array}$ & $\begin{array}{r}861.7 \\
2.12 \\
1.56 \\
0.56 \\
0.00 \\
2.32\end{array}$ & $\begin{array}{r}727.5 \\
2.22 \\
1.74 \\
0.48 \\
0.00 \\
2.42\end{array}$ & $\begin{array}{r}827.7 \\
2.23 \\
1.78 \\
0.45 \\
0.00 \\
2.43\end{array}$ & $\begin{array}{r}825.8 \\
1.90 \\
1.58 \\
0.32 \\
0.00 \\
2.10\end{array}$ & $\begin{array}{r}0.0 \\
0.00 \\
0.00 \\
0.00 \\
0.00 \\
0.00\end{array}$ & $\begin{array}{l}0.0 \\
0.00 \\
0.00 \\
0.00 \\
0.00 \\
0.00\end{array}$ & $25,600,002$ & $268,089,215$ \\
\hline
\end{tabular}

(1) Acts as agent for MASSPONER and Granite State Gas Transmission System
(2) Effective November 1, 1993, PGT tecame a transporter-only pipeline. 


\section{VOLUNG AMD PRICS RBPORT}

Long Term Imports

Volumes (MMcf/d) \& Prices (S/MMBTO) of Natural

Gas Imported During the Past 12 Months $01 / 01 / 93-12 / 31 / 93$

\section{Long-Tere Ieporter}

PAN MATIOML GAS SALES, INC. (1)

(SOMATRADING)

AVG Daily Quantity

Total Price (Lake Charles, Louisiana)

Commodity Component

Demand Component

Reservation fee Component

PAUTUCKET PONER ASSOCIATES

(TARRAGON OIL \& GAS LIMITED)

AVG Daily Quantity

Total Price (Haddington, New York)

Commodity Component

Denand Component

Reservation fee component

PAUTUCKET PONER ASSOCIATES

(HOME OIL COMPANY LIMITED)

AVG Daily Quantity

Total Price (Haddington, New York)

Commodity Component

Demand Component

Reservation fee component

PEOPLES MATURAL gaS COMPANY

(WGM LTD.)

AVG Daily Quantity

Total Price (Noyes, Minnesota)

Comodity Component

Demand Component

Reservation fee component

PEPPERELL PONER ASSOCIATES, L.P.

(WGM LTD.)

AVG Daily Quantity

Total Price (Niegara Falls, Mew York)

Commodity Component

Demand Component

Reservation Fee Component

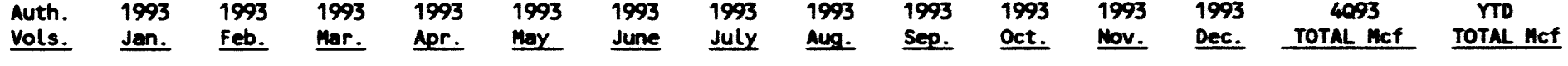

\begin{tabular}{|c|c|c|c|c|c|c|c|c|c|c|c|}
\hline $\begin{array}{r}0.0 \\
0.00 \\
0.00 \\
0.00 \\
0.00\end{array}$ & $\begin{array}{l}0.0 \\
0.00 \\
0.00 \\
0.00 \\
0.00\end{array}$ & $\begin{array}{l}0.0 \\
0.00 \\
0.00 \\
0.00 \\
0.00\end{array}$ & $\begin{array}{l}84.0 \\
1.89 \\
1.89 \\
0.00 \\
0.00\end{array}$ & $\begin{array}{l}81.1 \\
1.82 \\
1.82 \\
0.00 \\
0.00\end{array}$ & $\begin{array}{l}85.6 \\
1.75 \\
1.75 \\
0.00 \\
0.00\end{array}$ & $\begin{array}{r}163.9 \\
1.75 \\
1.75 \\
0.00 \\
0.00\end{array}$ & $\begin{array}{l}83.7 \\
1.75 \\
1.75 \\
0.00 \\
0.00\end{array}$ & $\begin{array}{r}167.7 \\
1.75 \\
1.75 \\
0.00 \\
0.00\end{array}$ & $\begin{array}{l}0.0 \\
0.00 \\
0.00 \\
0.00 \\
0.00\end{array}$ & $\begin{array}{l}70.9 \\
1.75 \\
1.75 \\
0.00 \\
0.00\end{array}$ & $\begin{array}{l}80.2 \\
1.75 \\
1.75 \\
0.00 \\
0.00\end{array}$ \\
\hline
\end{tabular}

$4,612,261 \quad 24,922,010$

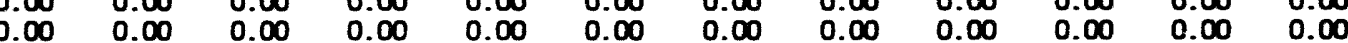

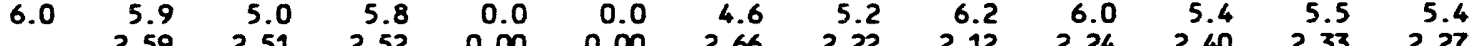
$\frac{2.59}{1.65} \frac{2.51}{1.57} \frac{2.52}{1.58} \frac{0.00}{0.00} \frac{0.00}{0.00} \frac{2.66}{1.69} \frac{2.22}{1.47} \frac{2.12}{1.47} \frac{2.24}{1.55} \frac{2.40}{1.55} \frac{2.33}{1.48} \frac{2.27}{1.42}$

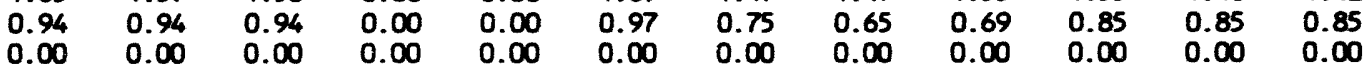

$500,730 \quad 1,681,802$

8.2 \begin{tabular}{rrrrrrrrrrrrr}
5.7 & 7.4 & 4.1 & 6.9 & 7.3 & 6.6 & 7.3 & 8.2 & 7.8 & 6.9 & 7.3 & 7.2 \\
& 2.56 & 2.44 & 2.50 & 2.71 & 2.90 & 2.69 & 2.23 & 2.17 & 2.28 & 2.44 & 2.31 & 2.30 \\
\hline 1.62 & 1.50 & 1.56 & 1.74 & 1.93 & 1.72 & 1.48 & 1.52 & 1.59 & 1.59 & 1.46 & 1.45 \\
0.94 & 0.94 & 0.94 & 0.97 & 0.97 & 0.97 & 0.75 & 0.65 & 0.69 & 0.85 & 0.85 & 0.85
\end{tabular}

$656,746 \quad 2,519,430$

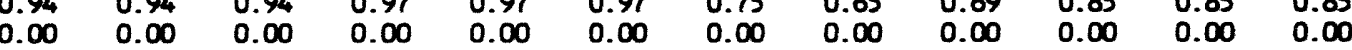

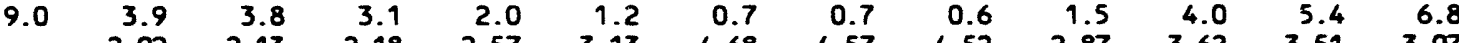

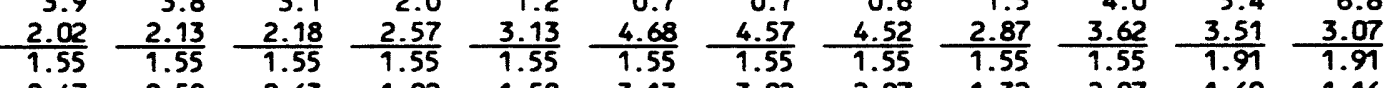

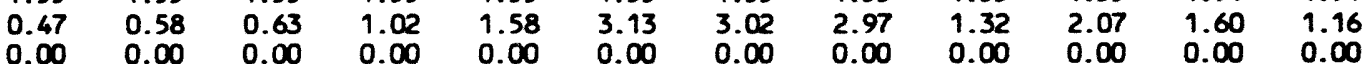

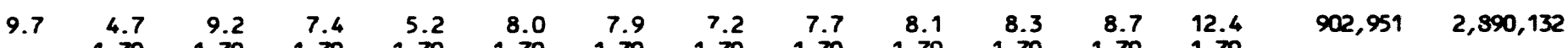

(1) Tailgate Price. 


\section{VOLUNT AND PRICE RBPORT}

\section{Volumes (Ming Term Import:}

Gas Imported During the Past 12 Months $01 / 01 / 93-12 / 31 / 93$

Long-Term Importer

PONER CITY PARTNERS, L.P.

(HUSKY OIL OPERATIONS LTD.)

AVG Daily Quantity

Total Price (Massena, Nevi York)

\section{Comadity Component}

Demand Component

Reservation Fee Component

PROGAS U.S.A. INC. (1)

(PROGAS LIMITED)

AVG Daily Quantity

Total Price (Noyes, Minnesota)

Commodity Component

Demand Component

Reservation fee Component

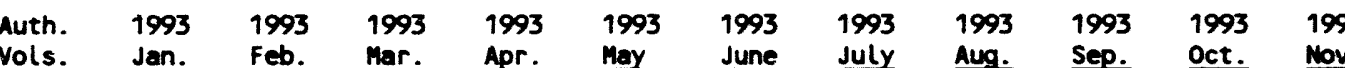

21

\begin{tabular}{llll}
.0 & 12.2 & 13.7 & 15.0 \\
& 7.03 & 3.56 & 3.14 \\
\cline { 4 - 4 } & 3.09 & 1.87 \\
& 3.14 & 1.53 & 1.27 \\
0.00 & 0.00 & 0.00
\end{tabular}

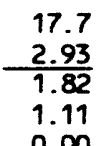

\begin{tabular}{lr}
17.7 & 16.8 \\
2.93 & $\frac{2.91}{1.82}$ \\
\hline 1.79 \\
.11 & 1.12 \\
0.00 & 0.00
\end{tabular}

$$
\begin{array}{r}
3.9 \\
4.17 \\
\hline 1.80 \\
2.37 \\
0.00
\end{array}
$$

$0.00 \quad 0.00$

8.0
3.26
1.81
1.45

$\frac{15.4}{2.99}$

$$
\frac{2}{1} \frac{1}{1}
$$

$\begin{array}{llll}16.0 & 16.9 & 18.0 & 18.1\end{array}$

$\frac{2.53}{1.38} \frac{3.21}{1.83} \frac{3.21}{1.85} \frac{3.18}{1.87}$

$\begin{array}{lllll}1.15 & 1.38 & 1.36 & 1.31\end{array}$

$\begin{array}{llll}0.00 & 0.00 & 0.00 & 0.00\end{array}$

75

$$
\begin{aligned}
& 0.00 \\
& 0.00 \\
& 0.00 \\
& 0.00
\end{aligned}
$$

\begin{tabular}{ccc}
0.0 & 0.0 & 0.0 \\
0.00 & 0.00 & 0.00 \\
\cline { 2 - 3 } & 0.00 & 0.00 \\
0.00 & 0.00 & 0.00 \\
0.00 & 0.00 & 0.00
\end{tabular}

\begin{tabular}{r}
0.0 \\
0.00 \\
\hline 0.00 \\
0.00 \\
0.00
\end{tabular}

0.0

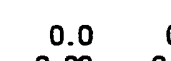

0.0

0

0.00

0.00

PROJECT ORANGE ASSOCIATES, L.P.

(NORANDA, INC.)

AVG Daily Quantity

Total Price (Niagara Falls, New York) Commodity Component

Reservation Fee Component

PUBLIC SERVICE ELECTRIC \& GAS

(ALBERTA NORTHEAST GAS (WGM LTD. 1))

AVG Daily Quantity

Total Price (Waddington, New York)

Commodity Component

Demand Component

Reservation fee Component

0.0

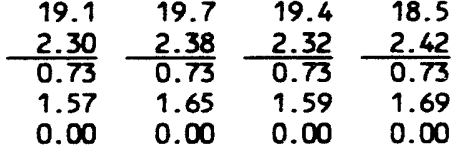

10.0

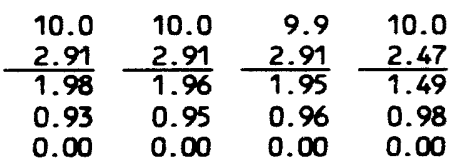

\begin{tabular}{l}
10.0 \\
2.70 \\
\hline 1.73 \\
0.97 \\
0.00
\end{tabular}

\begin{tabular}{lll}
10.0 & 10.0 & 10.0 \\
2.37 & 2.37 & 2.37 \\
\cline { 2 - 3 } & 1.39 & 1.40 \\
0.98 & 0.98 & 0.97 \\
0.00 & 0.00 & 0.00
\end{tabular}

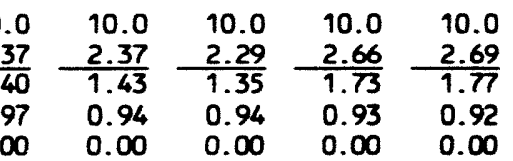

ROCHESTER GAS AND ELECTRIC CORPORATION

(WESTCOAST GAS SERVICES)

AVG Daily Quantity

Total Price (Grand Island, New York)

Commodity Component

Reservation Fee Component
16.0

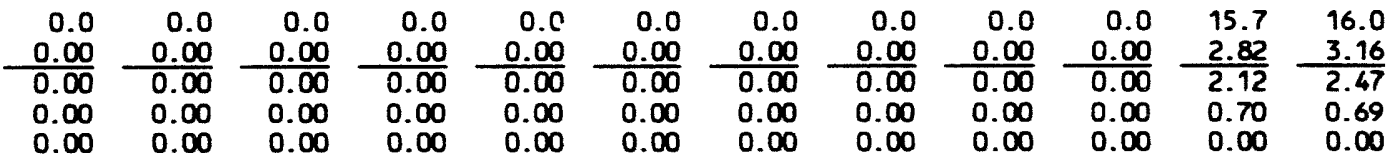

$1,626,042 \quad 5,239,970$

$6,900,000$

$6,900,000$

920,000

$3,656,699$

967,904

967,904

(1) Supply is resold to Consumers Power Company.

(2) Project Orange purchased 120,000,000 mmBtu's of natural gas over a 20-year period in one lump-sum amount totaling s88 million, or approximately $\mathbf{5 . 7 3}$ per MMBtu, for its 111 MW cogenerator in Syracuse, N.Y. The demand component includes Nova/TransCanada transportation; production, gathering, processing fees; and tax/royalty payments. 


\section{VOLUNE AND PRICE REPORT}

\section{Long Term Imports}

Volumes (MMcf/d) \& Prices (S/MMBTU) of Natural

Gas Imported During the Past 12 Months 01/01/93 - 12/31/93

\section{Long-Term Importer}

SALMON RESOURCES LTD. (1)

(SHELL CANADA LIMITED)

AVG Daily Quant ity

Total Price (Niagara Falls, New York)

Commodity Component

Demand Component

Reservation Fee Component

SALMON RESOURCES LTD. -ENRON GAS MARKETING (SHELL CANADA LIMITED)

AVG Daily Quantity

Total Price (Port of Morgan, Montana) Commodity Component

Demand Component

Reservation Fee Component

SALMON RESOURCES LTD. -MIDWEST GAS

(SHELL CANADA LIMITED)

AVG Daily Quantity

Total Price (Port of Morgan, Montana)

Commodity Component

Demand Component

Reservation Fee Component

SAN DIEGO GAS \& ELECTRIC COMPANY

(BOW VALLEY INDUSTRIES LTD.)

AVG Daily Quantity

Total Price (Eastport Idaho) Comood ity Component

Demand Component

Reservation Fee Component

SAN DIEGO GAS \& ELECTRIC COMPANY

(CANADIAN HUNTER MARKETING LTD.)

AVG Daily Quantity

rotal Price (Eastport, Idaho

Commodity Component

Demand Component

Reservation Fee Component
Auth. $1993 \quad 1993 \quad 1993 \quad 1993$

14.0

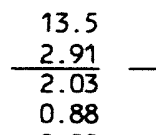

Feb.

Mar.

1993

1993

1993

1993

1993
Aug.

1993

Sep. Oct.

1993
Oct. Nov. Dec.

TAL Mcf

$$
0.88
$$$$
\begin{aligned}
& 14.4 \\
& 2.91 \\
& 1.98 \\
& 0.93 \\
& 0.00
\end{aligned}
$$$$
\begin{array}{lll}
14.0 & 14.5 & 14.5 \\
3.17 & 2.93 & 2.93 \\
\hline 2.30 & 2.01 & 2.05 \\
0.87 & 0.92 & 0.88 \\
0.00 & 0.00 & 0.00
\end{array}
$$$$
\begin{aligned}
& 13.8 \\
& 2.93 \\
& \hline 1.98 \\
& 0.95 \\
& 0.00
\end{aligned}
$$$$
\begin{array}{rr}
9.3 & 14 \\
2.93 & 2.89 \\
\cline { 2 - 2 } & 2.04 \\
1.36 & 0.85 \\
0.00 & 0.00
\end{array}
$$

\begin{tabular}{lll}
14.5 & $13.4 \quad 14.3$ \\
\cline { 2 - 3 }
\end{tabular}

$\begin{array}{lll}14.3 & 15.0 \quad 12.9\end{array}$

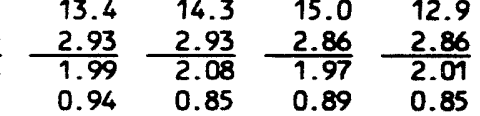

9.8

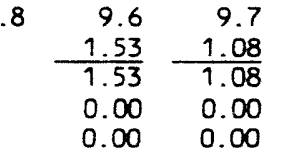

\begin{tabular}{rr}
9.6 & 9.5 \\
1.35 & $\frac{1.44}{1.35}$ \\
\hline 0.00 & 0.00 \\
0.00 & 0.00
\end{tabular}

$9.6 \quad 9.7$

\begin{tabular}{rrrrrrr}
.7 & 7.7 & 9.7 & 9.6 & 9.7 & 9.8 & 9.6 \\
23 & 1.23 & 1.33 & 1.77 & 1.67 & 1.64 & $\frac{1.83}{23}$ \\
\cline { 2 - 6 } & 1.23 & 1.33 & 1.77 & 1.67 & 1.64 & 1.83 \\
00 & 0.00 & 0.00 & 0.00 & 0.00 & 0.00 & 0.00 \\
00 & 0.00 & 0.00 & 0.00 & 0.00 & 0.00 & 0.00
\end{tabular}

891,127

$3,470,788$

20.

\begin{tabular}{lll}
0.5 & 18.3 & 20.4 \\
& 1.97 & 1.20 \\
\hline 1.97 & 1.20 \\
0.00 & 0.00 \\
0.00 & 0.00
\end{tabular}

\begin{tabular}{l}
20.2 \\
1.36 \\
\hline 1.36 \\
0.00 \\
0.00
\end{tabular}

\begin{tabular}{ll}
18.6 & 12.1 \\
1.50 & 2.24 \\
\hline 1.50 & 2.24 \\
0.00 & 0.00 \\
0.00 & 0.00
\end{tabular}

$20.4 \quad 18.9$

$17.6 \quad 16$.

20.1

20.1

16.9

$\begin{array}{ll}0.00 & 0.00 \\ 0.00 & 0.00\end{array}$

1.36
0.00
0.00

1.60
1.40
0.00

$\frac{1.80}{1.80} \frac{1.48}{1.48} \frac{1.69}{1.69} \frac{1.85}{1.85}$

$\infty$

4.9 \begin{tabular}{rrrrrrrrrrrrr}
0.0 & 0.0 & 0.0 & 0.0 & 0.0 & 0.0 & 0.0 & 0.0 & 0.0 & 0.0 & 4.9 & 4.0 \\
& 0.00 & 0.00 & 0.00 & 0.00 & 0.00 & 0.00 & 0.00 & 0.00 & 0.00 & 0.00 & $\frac{1.02}{1.46}$ & $\frac{1.46}{1.46}$ \\
\hline 0.00 & 0.00 & 0.00 & 0.00 & 0.00 & 0.00 & 0.00 & 0.00 & 0.00 & 0.00 & 1.02 & 0.00 \\
0.00 & 0.00 & 0.00 & 0.00 & 0.00 & 0.00 & 0.00 & 0.00 & 0.00 & 0.00 & 0.00 & 0.00 \\
0.00 & 0.00 & 0.00 & 0.00 & 0.00 & 0.00 & 0.00 & 0.00 & 0.00 & 0.00 & 0.00 & 0.00
\end{tabular}

19.7

\begin{tabular}{rrrrrrrrrrrrr}
0.0 & 0.0 & 0.0 & 0.0 & 0.0 & 0.0 & 0.0 & 0.0 & 0.0 & 0.0 & 19.7 & 19.7 \\
0.00 & 0.00 & 0.00 & 0.00 & 0.00 & 0.00 & 0.00 & 0.00 & 0.00 & 0.00 & $\frac{1.03}{1.03}$ & $\frac{1.48}{1.48}$ \\
\hline 0.00 & 0.00 & 0.00 & 0.00 & 0.00 & 0.00 & 0.00 & 0.00 & 0.00 & 0.00 & 1.03 & 1.48 \\
0.00 & 0.00 & 0.00 & 0.00 & 0.00 & 0.00 & 0.00 & 0.00 & 0.00 & 0.00 & 0.00 & 0.00 \\
0.00 & 0.00 & 0.00 & 0.00 & 0.00 & 0.00 & 0.00 & 0.00 & 0.00 & 0.00 & 0.00 & 0.00
\end{tabular}

272,158

272,158

$1,748,818 \quad 6,694,089$

$1,202,416 \quad 1,202,416$

(1) Gas resold to Cogen Energy Technology, Inc. to fuel a cogeneration facility at Castleton-on-Hudson, N.Y. 


\section{VOLUNE AKD PRICE REPORI}

\section{Long Term Imports}

Volumes (MMCf/d) \& Prices (S/MMBTU) of Natura

Gas Imported During the Past 12 Months 01/01/93 - 12/31/93

\section{Long-Term Importer}

SAN DIEGO GAS \& ELECTRIC COMPANY (HUSKY OIL OPERATIONS LTD.)

AVG Daily Quantity

Total Price (Eastport, Idaho)

Commodity Component

Demand Component

Reservation Fee Component

SAN DIEGO GAS \& ELECTRIC COMPANY

(SUMMIT RESOURCES LTT.)

AVG Daily Quantity

Total Price (Eastport, Idaho)

Conmodity Component

Reservation Fee Component

SARANAC POWER PARTNERS, L.P.

(SHELL CANADA LIMITED)

AVG Daily Quantity

Total Price (Champlain, New York

Commodity Component

Demand Component

Reservation Fee Component

SELKIRK COGEN PARTNERS, L.P. (1)

(PARAMOUNT RESOURCES LTD.)

AVG Daily Quantity

Total Price (Waddington, New York)

Commodity Component

Reservation Fee Component

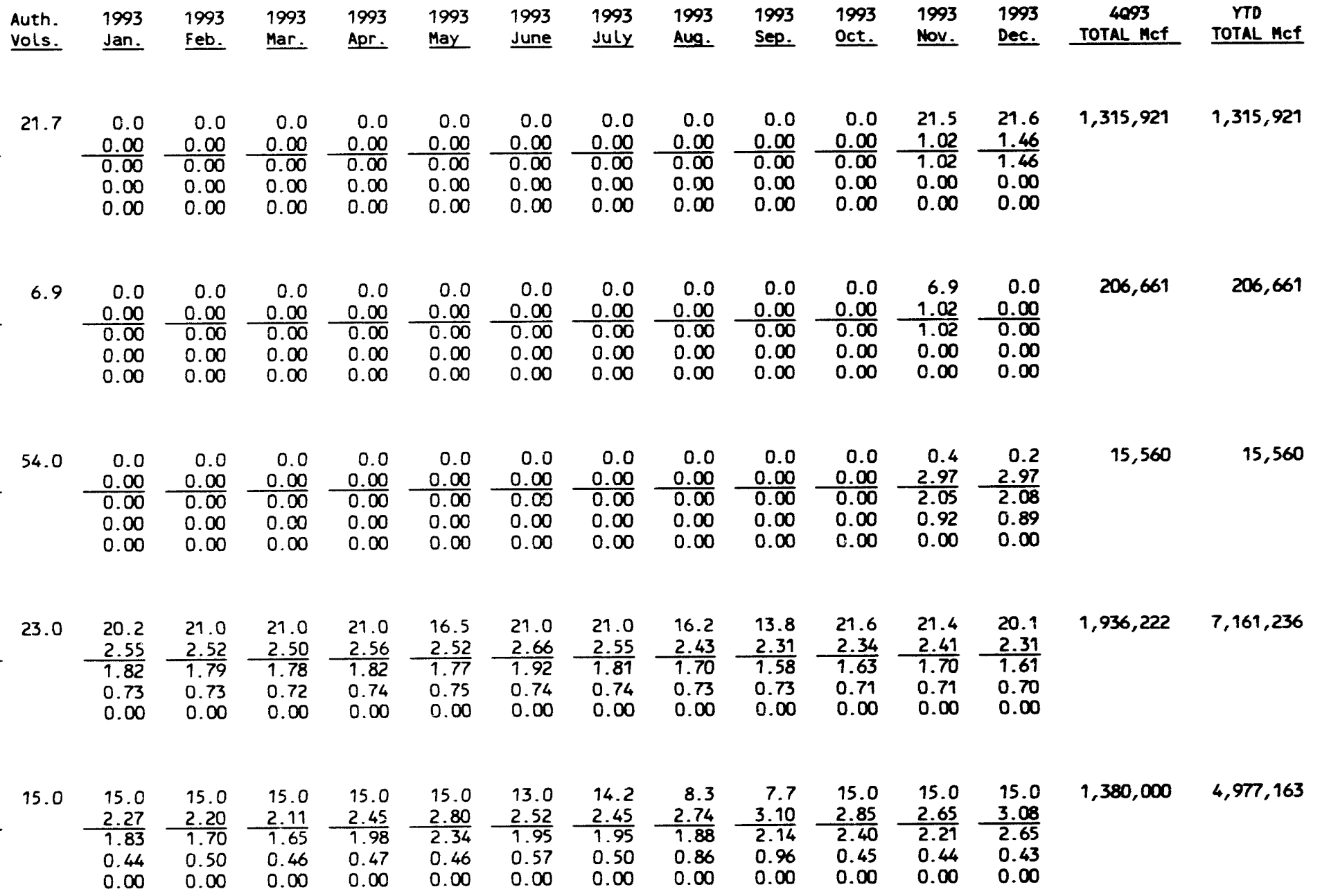

MICHIGAN GAS COMPANY

(WGM LTD.)

AVG Daily Quantity

Total Frice (Noyes, Minnesota)

Commodity Componen

Reservation Fee Component

$\begin{array}{lll}0.00 & 0.00 & 0.00\end{array}$

(1) Selkirk's 2nd and 3rd quarter volumes include 363,345 Mcf imported during May, June, and July at Niagara falls, New York at the above listed prices. 


\section{VOLUIE AND PRICE REPORT}

Iong Term Imports

Volunes (MMCf/d) \& Prices (S/MMBTU) of Natural

Gas Imported During the Past 12 Months $01 / 01 / 93$ - 12/31/93

\begin{tabular}{|c|c|c|c|c|c|c|c|c|c|c|c|c|c|c|c|}
\hline Long-Tere Importer & $\begin{array}{l}\text { Auth. } \\
\text { Vols. }\end{array}$ & $\begin{array}{l}1993 \\
\text { Jan. }\end{array}$ & $\begin{array}{l}1993 \\
\text { Feb. }\end{array}$ & $\begin{array}{l}1993 \\
\text { Mar. }\end{array}$ & $\begin{array}{l}1993 \\
\text { Apr. }\end{array}$ & $\begin{array}{l}1993 \\
\text { May }\end{array}$ & $\begin{array}{l}1993 \\
\text { June } \\
\end{array}$ & $\begin{array}{l}1993 \\
\text { July } \\
\end{array}$ & $\begin{array}{l}1993 \\
\text { Aug. }\end{array}$ & $\begin{array}{l}1993 \\
\text { Sep. }\end{array}$ & $\begin{array}{l}1993 \\
\text { oct. }\end{array}$ & $\begin{array}{l}1993 \\
\text { Nov. }\end{array}$ & $\begin{array}{l}1993 \\
\text { Dec. }\end{array}$ & $\begin{array}{c}4093 \\
\text { TOTAL MCf } \\
\end{array}$ & $\begin{array}{l}\text { YTD } \\
\text { TOTAL MCf } \\
\end{array}$ \\
\hline $\begin{array}{l}\text { SOUTHERN CALIFORNIA EDISON COMPANY } \\
\text { (SHELL CANADA LIMITED) } \\
\text { AVG Daily Quantity } \\
\text { Total Price (Eastport, Idaho) } \\
\text { Commodity Component } \\
\text { Demand Component } \\
\text { Reservation Fee Component }\end{array}$ & 51.0 & $\begin{array}{r}0.0 \\
0.00 \\
0.00 \\
0.00 \\
0.00\end{array}$ & $\begin{array}{l}0.0 \\
0.00 \\
0.00 \\
0.00 \\
0.00\end{array}$ & $\begin{array}{r}0.0 \\
0.00 \\
0.00 \\
0.00 \\
0.00\end{array}$ & $\begin{array}{r}0.0 \\
0.00 \\
0.00 \\
0.00 \\
0.00\end{array}$ & $\begin{array}{r}0.0 \\
0.00 \\
0.00 \\
0.00 \\
0.00\end{array}$ & $\begin{array}{l}0.0 \\
0.00 \\
0.00 \\
0.00 \\
0.00\end{array}$ & $\begin{array}{r}0.0 \\
0.00 \\
0.00 \\
0.00 \\
0.00\end{array}$ & $\begin{array}{r}0.0 \\
0.00 \\
0.00 \\
0.00 \\
0.00\end{array}$ & $\begin{array}{r}0.0 \\
0.00 \\
0.00 \\
0.00 \\
0.00\end{array}$ & $\begin{array}{r}0.0 \\
0.00 \\
0.00 \\
0.00 \\
0.00\end{array}$ & $\begin{array}{l}51.6 \\
1.39 \\
1.34 \\
0.05 \\
0.00\end{array}$ & $\begin{array}{l}49.5 \\
1.58 \\
1.53 \\
0.05 \\
0.00\end{array}$ & $3,082,412$ & $3,082,412$ \\
\hline $\begin{array}{l}\text { SOUTHERN CALIFORNIA EDISON COMPANY } \\
\text { (AEC OIL \& GAS COMPANY) } \\
\text { AVG Daily Quant ity } \\
\text { Total Price (Eastport, Idaho) } \\
\text { Commodity Component } \\
\text { Demand Component } \\
\text { Reservation Fee Component }\end{array}$ & 51.8 & $\begin{array}{r}0.0 \\
0.00 \\
0.00 \\
0.00 \\
0.00\end{array}$ & $\begin{array}{r}0.0 \\
0.00 \\
0.00 \\
0.00 \\
0.00\end{array}$ & $\begin{array}{r}0.0 \\
0.00 \\
0.00 \\
0.00 \\
0.00\end{array}$ & $\begin{array}{r}0.0 \\
0.00 \\
0.00 \\
0.00 \\
0.00\end{array}$ & $\begin{array}{r}0.0 \\
0.00 \\
0.00 \\
0.00 \\
0.00\end{array}$ & $\begin{array}{r}0.0 \\
0.00 \\
0.00 \\
0.00 \\
0.00\end{array}$ & $\begin{array}{l}0.0 \\
0.00 \\
0.00 \\
0.00 \\
0.00\end{array}$ & $\begin{array}{r}0.0 \\
0.00 \\
0.00 \\
0.00 \\
0.00\end{array}$ & $\begin{array}{r}0.0 \\
0.00 \\
0.00 \\
0.00 \\
0.00\end{array}$ & $\begin{array}{r}0.0 \\
0.00 \\
0.00 \\
0.00 \\
0.00\end{array}$ & $\begin{array}{l}35.3 \\
1.31 \\
1.26 \\
0.05 \\
0.00\end{array}$ & $\begin{array}{l}34.7 \\
1.47 \\
1.42 \\
0.05 \\
0.00\end{array}$ & $2,132,144$ & $2,132,144$ \\
\hline $\begin{array}{l}\text { SOUTHERN CALIFORNIA EDISON COMPANY } \\
\text { (IMPERIAL OIL RESOURCES LIMITED) } \\
\text { AVG Daily Quant ity } \\
\text { Total Price (Eastport, Idaho) } \\
\text { Commodity Compor snt } \\
\text { Demand Component } \\
\text { Reservation Fee Component }\end{array}$ & 51.8 & $\begin{array}{l}0.0 \\
0.00 \\
0.00 \\
0.00 \\
0.00\end{array}$ & $\begin{array}{r}0.0 \\
0.00 \\
0.00 \\
0.00 \\
0.00\end{array}$ & $\begin{array}{r}0.0 \\
0.00 \\
0.00 \\
0.00 \\
0.00\end{array}$ & $\begin{array}{l}0.0 \\
0.00 \\
0.00 \\
0.00 \\
0.00\end{array}$ & $\begin{array}{r}0.0 \\
0.00 \\
0.00 \\
0.00 \\
0.00\end{array}$ & $\begin{array}{l}0.0 \\
0.00 \\
0.00 \\
0.00 \\
0.00\end{array}$ & $\begin{array}{l}0.0 \\
0.00 \\
0.00 \\
0.00 \\
0.00\end{array}$ & $\begin{array}{l}0.0 \\
0.00 \\
0.00 \\
0.00 \\
0.00\end{array}$ & $\begin{array}{r}0.0 \\
0.00 \\
0.00 \\
0.00 \\
0.00\end{array}$ & $\begin{array}{r}0.0 \\
0.00 \\
0.00 \\
0.00 \\
0.00\end{array}$ & $\begin{array}{l}25.2 \\
1.31 \\
1.26 \\
0.05 \\
0.00\end{array}$ & $\begin{array}{l}24.8 \\
1.47 \\
1.42 \\
0.05 \\
0.00\end{array}$ & $1,522,960$ & $1,522,960$ \\
\hline $\begin{array}{l}\text { SOUTHERN CALIFORNIA EDISON COMPANY } \\
\text { (WGM LTD.) } \\
\text { AVG Daily Quantity } \\
\text { Total Price (Eastport, Idaho) } \\
\text { Commodity Component } \\
\text { Demand Component } \\
\text { Reservation Fee Component }\end{array}$ & 51.8 & $\begin{array}{r}0.0 \\
0.00 \\
0.00 \\
0.00 \\
0.00\end{array}$ & $\begin{array}{r}0.0 \\
0.00 \\
0.00 \\
0.00 \\
0.00\end{array}$ & $\begin{array}{r}0.0 \\
0.00 \\
0.00 \\
0.00 \\
0.00\end{array}$ & $\begin{array}{r}0.0 \\
0.00 \\
0.00 \\
0.00 \\
0.00\end{array}$ & $\begin{array}{r}0.0 \\
0.00 \\
0.00 \\
0.00 \\
0.00\end{array}$ & $\begin{array}{r}0.0 \\
0.00 \\
0.00 \\
0.00 \\
0.00\end{array}$ & $\begin{array}{r}0.0 \\
0.00 \\
0.00 \\
0.00 \\
0.00\end{array}$ & $\begin{array}{r}0.0 \\
0.00 \\
0.00 \\
0.00 \\
0.00\end{array}$ & $\begin{array}{r}0.0 \\
0.00 \\
0.00 \\
0.00 \\
0.00\end{array}$ & $\begin{array}{r}0.0 \\
0.00 \\
0.00 \\
0.00 \\
0.00\end{array}$ & $\begin{array}{l}35.3 \\
1.31 \\
1.26 \\
0.05 \\
0.00\end{array}$ & $\begin{array}{l}33.3 \\
1.47 \\
1.42 \\
0.05 \\
0.00\end{array}$ & $2,089,863$ & $2,089,863$ \\
\hline $\begin{array}{l}\text { SOUTHERN CONNECTICUT GAS } \\
\text { (ALBERTA NORTHEAST GAS (WGM LTD. 1)) } \\
\text { AVG Daily Quantity } \\
\text { Total Price (Waddington, New York) } \\
\text { Commodity Component } \\
\text { Demand Component } \\
\text { Reservation Fee Component }\end{array}$ & 15.0 & $\begin{array}{l}15.0 \\
2.91 \\
1.98 \\
0.93 \\
0.00\end{array}$ & $\begin{array}{l}15.0 \\
2.91 \\
1.96 \\
0.95 \\
0.00\end{array}$ & $\begin{array}{l}15.0 \\
2.91 \\
1.95 \\
0.96 \\
0.00\end{array}$ & $\begin{array}{l}15.0 \\
2.47 \\
1.49 \\
0.98 \\
0.00\end{array}$ & $\begin{array}{l}15.0 \\
2.70 \\
1.73 \\
0.97 \\
0.00\end{array}$ & $\begin{array}{l}15.0 \\
2.37 \\
1.39 \\
0.98 \\
0.00\end{array}$ & $\begin{array}{l}15.0 \\
2.37 \\
1.39 \\
0.98 \\
0.00\end{array}$ & $\begin{array}{l}15.0 \\
2.37 \\
1.40 \\
0.97 \\
0.00\end{array}$ & $\begin{array}{l}15.0 \\
2.37 \\
1.43 \\
0.94 \\
0.00\end{array}$ & $\begin{array}{l}15.0 \\
2.29 \\
1.35 \\
0.94 \\
0.00\end{array}$ & $\begin{array}{l}15.0 \\
2.66 \\
1.73 \\
0.93 \\
0.00\end{array}$ & $\begin{array}{l}15.0 \\
2.69 \\
1.77 \\
0.92 \\
0.00\end{array}$ & $1,380,000$ & $5,490,000$ \\
\hline
\end{tabular}




\section{VOLUNIE AND PRICE REPORT}

Iong Tere Imports

Volumes (MMCf/d) \& Prices (S/MMBTU) of Natural

Gas Imported During the Past 12 Months 01/01/93 - 12/31/93

\section{Long-Term Importer}

SOUTHERN CONNECTICUT GAS (ALBERTA NORTHEAST GAS (HGM LTD. 2)) AVG Daily Quantity

Total Price (Waddington, New York)

Commodity Component

Demand Component

Reservation Fee Component

SOUTHERN CONNECTICUT GAS

(ALBERTA NORTHEAST GAS (PROGAS))

AVG Daily Quantity

Total Price (Waddington, New York)

Commodity Component

Demand Component

Reservation Fee Component

SOUTHERN CONNECTICUT GAS

(ALBERTA NORTHEAST GAS (ATCOR))

AVG Daily Quantity

Total Price (Waddington, New York)

Commodity Componerit

Demand Component

Reservation fee Component

SOUTHERN CONNECTICUT GAS

(ALBERTA NE GAS (AEC OIL \& GAS COMPANY))

(ALBERTA NE GAS (AE
AVG Daily Quantity

Total Price (Waddington, New York)

Commodity Componen

Demand Component

Reservation Fee Component

ST. LAWRENCE GAS COMPANY INC.

(NIAGARA GAS TRANSMISSION LTD.)

AVG Daily Quantity

Total Price (Massena, New York)

Commodity Component

Demand Component

Reservation Fee Component

\begin{tabular}{|c|c|c|c|c|c|c|c|c|c|c|c|c|c|c|}
\hline $\begin{array}{l}\text { Auth. } \\
\text { Vols. }\end{array}$ & $\begin{array}{l}1993 \\
\text { Jan. }\end{array}$ & $\begin{array}{l}1993 \\
\text { Feb. }\end{array}$ & $\begin{array}{l}1993 \\
\text { Mar. }\end{array}$ & $\begin{array}{l}1993 \\
\text { Apr. }\end{array}$ & $\begin{array}{l}1993 \\
\text { May }\end{array}$ & $\begin{array}{l}1993 \\
\text { June }\end{array}$ & $\begin{array}{l}1993 \\
\text { July } \\
\end{array}$ & $\begin{array}{l}1993 \\
\text { Aug. }\end{array}$ & $\begin{array}{l}1993 \\
\text { Sep. }\end{array}$ & $\begin{array}{l}1993 \\
\text { Oct. }\end{array}$ & $\begin{array}{l}1993 \\
\text { Nov. } \\
\text {. }\end{array}$ & $\begin{array}{l}1993 \\
\text { Dec. }\end{array}$ & $\begin{array}{c}4093 \\
\text { TOTAL MCf }\end{array}$ & $\begin{array}{c}\text { YTD } \\
\text { TOTAL MCf } \\
\end{array}$ \\
\hline 7.5 & $\begin{array}{l}7.3 \\
2.91 \\
1.98 \\
0.93 \\
0.00\end{array}$ & $\begin{array}{l}7.5 \\
2.91 \\
1.96 \\
0.95 \\
0.00\end{array}$ & $\begin{array}{l}7.5 \\
2.91 \\
1.95 \\
0.96 \\
0.00\end{array}$ & $\begin{array}{l}7.5 \\
2.47 \\
1.49 \\
0.98 \\
0.00\end{array}$ & $\begin{array}{r}7.5 \\
2.70 \\
1.73 \\
0.97 \\
0.00\end{array}$ & $\begin{array}{l}7.5 \\
2.37 \\
1.39 \\
0.98 \\
0.00\end{array}$ & $\begin{array}{r}7.5 \\
2.37 \\
1.39 \\
0.98 \\
0.00\end{array}$ & $\begin{array}{r}7.5 \\
2.37 \\
1.40 \\
0.97 \\
0.00\end{array}$ & $\begin{array}{r}7.5 \\
2.37 \\
1.43 \\
0.94 \\
0.00\end{array}$ & $\begin{array}{l}7.5 \\
2.29 \\
1.35 \\
0.94 \\
0.00\end{array}$ & $\begin{array}{r}7.5 \\
2.66 \\
1.73 \\
0.93 \\
0.00\end{array}$ & $\begin{array}{l}7.5 \\
2.69 \\
1.77 \\
0.92 \\
0.00\end{array}$ & 690,000 & $2,740,000$ \\
\hline 6.6 & $\begin{array}{r}6.6 \\
2.91 \\
1.97 \\
0.94 \\
0.00\end{array}$ & $\begin{array}{l}6.6 \\
2.91 \\
1.95 \\
0.96 \\
0.00\end{array}$ & $\begin{array}{l}6.6 \\
2.91 \\
1.97 \\
0.94 \\
0.00\end{array}$ & $\begin{array}{r}6.6 \\
2.46 \\
1.48 \\
0.98 \\
0.00\end{array}$ & $\begin{array}{r}6.6 \\
2.76 \\
1.78 \\
0.98 \\
0.00\end{array}$ & $\begin{array}{r}6.5 \\
2.37 \\
1.39 \\
0.98 \\
0.00\end{array}$ & $\begin{array}{r}6.6 \\
2.37 \\
1.38 \\
0.99 \\
0.00\end{array}$ & $\begin{array}{r}6.6 \\
2.37 \\
1.40 \\
0.97 \\
0.00\end{array}$ & $\begin{array}{r}6.6 \\
2.36 \\
1.42 \\
0.94 \\
0.00\end{array}$ & $\begin{array}{r}6.6 \\
2.30 \\
1.35 \\
0.95 \\
0.00\end{array}$ & $\begin{array}{r}6.6 \\
2.67 \\
1.73 \\
0.94 \\
0.00\end{array}$ & $\begin{array}{r}6.6 \\
2.69 \\
1.73 \\
0.96 \\
0.00\end{array}$ & 607,200 & $2,415,600$ \\
\hline 2.2 & $\begin{array}{r}2.2 \\
2.91 \\
2.02 \\
0.89 \\
0.00\end{array}$ & $\begin{array}{r}2.2 \\
2.92 \\
2.01 \\
0.91 \\
0.00\end{array}$ & $\begin{array}{r}2.2 \\
2.91 \\
2.01 \\
0.90 \\
0.00\end{array}$ & $\begin{array}{r}2.2 \\
2.46 \\
1.53 \\
0.93 \\
0.00\end{array}$ & $\begin{array}{r}2.2 \\
2.71 \\
1.75 \\
0.96 \\
0.00\end{array}$ & $\begin{array}{r}2.2 \\
2.37 \\
1.43 \\
0.94 \\
0.00\end{array}$ & $\begin{array}{r}2.0 \\
2.37 \\
1.45 \\
0.92 \\
0.00\end{array}$ & $\begin{array}{r}2.2 \\
2.37 \\
1.42 \\
0.95 \\
0.00\end{array}$ & $\begin{array}{r}2.2 \\
2.37 \\
1.48 \\
0.89 \\
0.00\end{array}$ & $\begin{array}{r}2.2 \\
2.29 \\
1.47 \\
0.82 \\
0.00\end{array}$ & $\begin{array}{r}2.2 \\
2.66 \\
1.78 \\
0.88 \\
0.00\end{array}$ & $\begin{array}{r}2.2 \\
2.68 \\
1.79 \\
0.89 \\
0.00\end{array}$ & 199,640 & 789,220 \\
\hline 43.0 & $\begin{array}{l}23.2 \\
2.06 \\
2.06 \\
0.00 \\
0.00\end{array}$ & $\begin{array}{l}23.2 \\
2.52 \\
2.52 \\
0.00 \\
0.00\end{array}$ & $\begin{array}{l}21.1 \\
1.88 \\
1.88 \\
0.00 \\
0.00\end{array}$ & $\begin{array}{l}16.6 \\
2.41 \\
2.41 \\
0.00 \\
0.00\end{array}$ & $\begin{array}{r}9.7 \\
3.84 \\
3.84 \\
0.00 \\
0.00\end{array}$ & $\begin{array}{r}8.3 \\
4.65 \\
4.65 \\
0.00 \\
0.00\end{array}$ & $\begin{array}{r}6.1 \\
2.19 \\
2.19 \\
0.00 \\
0.00\end{array}$ & $\begin{array}{r}6.8 \\
2.26 \\
2.26 \\
0.00 \\
0.00\end{array}$ & $\begin{array}{r}9.3 \\
2.20 \\
2.20 \\
0.00 \\
0.00\end{array}$ & $\begin{array}{l}14.5 \\
2.21 \\
2.21 \\
0.00 \\
0.00\end{array}$ & $\begin{array}{l}18.3 \\
3.15 \\
3.15 \\
0.00 \\
0.00\end{array}$ & $\begin{array}{l}22.6 \\
2.93 \\
2.93 \\
0.00 \\
0.00\end{array}$ & $1,697,818$ & $5,471,478$ \\
\hline
\end{tabular}




\section{VOLUNE AND PRICE REPORT}

\section{Long Term Imports}

Volumes (MMCf/d) \& Prices (S/MMBTU) of Natural

Gas Imported During the Past 12 Months $01 / 01 / 93-12 / 31 / 93$

\section{Long-Term Importer}

SUMAS COGENERATION COMPANY, L.P. (ENCO GAS, LTD.)

AVG Daily Quantity

Total Price (Sumas, Washington)

Commodity Component

Demand Component

Reservation Fee Component

SUMAS COGENERATION COMPANY, L.P.

(CANADIAN HYDROCARBONS MARKETING)

AVG Daily Quantity

Total Price (Sumas, washington)

Commodity component

emand Component

Reservation Fee Component

TENNESSEE GAS PIPELINE COMPANY

(PROGAS LIMITED)

AVG Daily Quantity

Total Price (Noyes, Minnesota)

Commodity Component

Demand Component

Reservation Fee Component

Auth. $1993 \quad 1993 \quad 1993 \quad 1993$

Auth.

1993
Apr.

12.0

$\begin{array}{ll}0.0 & \\ 0.00 & 0 . \\ 0.00 & 0.00 \\ 0.00 & 0.00 \\ 0.00 & 0.00\end{array}$

12.0

\begin{tabular}{cc}
0.0 & 0.0 \\
0.00 & 0.00 \\
\cline { 2 - 2 } 0.00 & 0.00 \\
0.00 & 0.00 \\
0.00 & 0.00
\end{tabular}

75.0

\begin{tabular}{ll}
32.2 & 28.8 \\
2.67 & 2.70 \\
\hline 1.85 & 1.69 \\
0.82 & 1.01 \\
0.00 & 0.00
\end{tabular}

\begin{tabular}{|l}
28.6 \\
2.75 \\
\hline 1.83 \\
0.92 \\
0.00
\end{tabular}

100.0

(KANNGAZ PRODUCERS LTD.)

(1)

AVG Daily Quantity

Total Price (Niagara Falls, New York) Commodity component

Reservation Fee Component

\begin{tabular}{|c|c|c|c|c|c|c|c|c|c|c|}
\hline $\begin{array}{r}0.0 \\
0.00 \\
0.00 \\
0.00 \\
0.00\end{array}$ & $\begin{array}{l}15.7 \\
5.40 \\
1.59 \\
3.81 \\
0.00\end{array}$ & $\begin{array}{l}25.0 \\
3.76 \\
1.84 \\
1.92 \\
0.00\end{array}$ & $\begin{array}{l}25.0 \\
4.51 \\
2.13 \\
2.38 \\
0.00\end{array}$ & $\begin{array}{l}25.0 \\
6.50 \\
2.64 \\
3.86 \\
0.00\end{array}$ & $\begin{array}{l}13.3 \\
5.79 \\
2.01 \\
3.78 \\
0.00\end{array}$ & $\begin{array}{r}6.5 \\
3.80 \\
1.89 \\
1.91 \\
0.00\end{array}$ & $\begin{array}{l}25.0 \\
4.15 \\
2.01 \\
2.14 \\
0.00\end{array}$ & $\begin{array}{l}25.0 \\
4.79 \\
2.29 \\
2.50 \\
0.00\end{array}$ & $\begin{array}{l}25.0 \\
3.78 \\
1.94 \\
1.84 \\
0.00\end{array}$ & $\begin{array}{l}25.0 \\
4.89 \\
2.07 \\
2.82 \\
0.00\end{array}$ \\
\hline $\begin{array}{l}16.5 \\
2.45 \\
1.88 \\
0.57 \\
0.00\end{array}$ & $\begin{array}{l}16.8 \\
2.21 \\
1.59 \\
0.62 \\
0.00\end{array}$ & $\begin{array}{l}16.7 \\
2.39 \\
1.84 \\
0.55 \\
0.00\end{array}$ & $\begin{array}{l}16.7 \\
2.72 \\
2.13 \\
0.59 \\
0.00\end{array}$ & $\begin{array}{l}14.7 \\
3.20 \\
2.64 \\
0.56 \\
0.00\end{array}$ & $\begin{array}{l}16.7 \\
2.60 \\
2.01 \\
0.59 \\
0.00\end{array}$ & $\begin{array}{l}16.7 \\
2.44 \\
1.89 \\
0.55 \\
0.00\end{array}$ & $\begin{array}{l}16.7 \\
2.57 \\
2.01 \\
0.56 \\
0.00\end{array}$ & $\begin{array}{r}16.9 \\
2.91 \\
2.22 \\
0.69 \\
0.00\end{array}$ & $\begin{array}{l}16.9 \\
2.53 \\
1.87 \\
0.66 \\
0.00\end{array}$ & $\begin{array}{l}16.9 \\
2.64 \\
2.00 \\
0.64 \\
0.00\end{array}$ \\
\hline
\end{tabular}

TENNESSEE GAS PIPELINE COMPANY (2)

(TRANSCANADA PIPELINES LIMITED)

AVG Daily Quantity

Demand Component

Reservation Fee Component
Total Price (Niagara Falls, New York)

Commodity Component $\begin{array}{llllllll}1993 & 1993 & 1993 & 1993 & 1993 & 1993 & 1993 & 1993 \\ \text { lay } & \text { June } & \text { July } & \text { Aug. } & \text { Sep. } & \text { Oct. } & \text { Nov. } & \text { Dec. }\end{array}$

c.

TOTAL Mcf

$2,748,108$

$10.7 \quad 13.0$ $\frac{2.10}{2.10} \frac{2.10}{2.10}$ $0.00 \quad 0.00$

890,044

$2,870,473$

$4,719,839$

$2,300,000$

$7,180,665$

$1,555,869$

$6,069,016$

(1) Demand charges for May include revisions of the monthly demand charge rates for January through December 1992 , which total to $\$ 1,163,001.75$.

(1) Demand charges for May include revisions of the monthly demand charge rates for January January through August 1993.

(2) Gas imported on behalf of assignees: 4th Quarter -- Penn Gas \& Water $(1,372,850)$, Penn Southern (183,019). 
VOLUNE AND PRICE REPORT

Long Term Imports

Volumes (MMCf/d) \& Prices (S/MMBTU) of Natural

Gas Imported During the Past 12 Months 01/01/93-12/31/93

\begin{tabular}{|c|c|c|c|c|c|c|c|c|c|c|c|c|c|c|c|}
\hline Long-Term Importer & $\begin{array}{l}\text { Auth. } \\
\text { Vols. }\end{array}$ & $\begin{array}{l}1993 \\
\text { Jan. }\end{array}$ & $\begin{array}{l}1993 \\
\text { Feb. }\end{array}$ & $\begin{array}{l}1993 \\
\text { Mar. }\end{array}$ & $\begin{array}{l}1993 \\
\text { Apr. }\end{array}$ & $\begin{array}{l}1993 \\
\text { May }\end{array}$ & $\begin{array}{l}1993 \\
\text { June }\end{array}$ & $\begin{array}{l}1993 \\
\text { July } \\
\end{array}$ & $\begin{array}{l}1993 \\
\text { Aug. }\end{array}$ & $\begin{array}{l}1993 \\
\text { Sep. }\end{array}$ & $\begin{array}{l}1993 \\
\text { Oct. }\end{array}$ & $\begin{array}{l}1993 \\
\text { Nov. }\end{array}$ & $\begin{array}{l}1993 \\
\text { Dec. }\end{array}$ & $\begin{array}{c}4093 \\
\text { TOTAL Mcf }\end{array}$ & $\begin{array}{c}\text { YTD } \\
\text { TOTAL MCf } \\
\end{array}$ \\
\hline $\begin{array}{l}\text { TEXAS EASTERN TRANSMISSION CORPORATION } \\
\text { (PROGAS LIMITED) } \\
\text { AVG Daily Quantity } \\
\text { Total Price (Noyes, Minnesota) } \\
\text { Commodity Component } \\
\text { Demand Componert } \\
\text { Reservation Fee Component }\end{array}$ & 75.0 & $\begin{array}{r}0.0 \\
0.00 \\
0.00 \\
0.00 \\
0.00\end{array}$ & $\begin{array}{r}0.0 \\
0.00 \\
0.00 \\
0.00 \\
0.00\end{array}$ & $\begin{array}{l}16.1 \\
3.18 \\
1.48 \\
1.70 \\
0.00\end{array}$ & $\begin{array}{r}0.0 \\
0.00 \\
0.00 \\
0.00 \\
0.00\end{array}$ & $\begin{array}{r}0.0 \\
0.00 \\
0.00 \\
0.00 \\
0.00\end{array}$ & $\begin{array}{r}0.0 \\
0.00 \\
0.00 \\
0.00 \\
0.00\end{array}$ & $\begin{array}{r}0.0 \\
0.00 \\
0.00 \\
0.00 \\
0.00\end{array}$ & $\begin{array}{r}0.0 \\
0.00 \\
0.00 \\
0.00 \\
0.00\end{array}$ & $\begin{array}{r}0.0 \\
0.00 \\
0.00 \\
0.00 \\
0.00\end{array}$ & $\begin{array}{l}0.0 \\
0.00 \\
0.00 \\
0.00 \\
0.00\end{array}$ & $\begin{array}{r}0.0 \\
0.00 \\
0.00 \\
0.00 \\
0.00\end{array}$ & $\begin{array}{r}0.0 \\
0.00 \\
0.00 \\
0.00 \\
0.00\end{array}$ & 0 & 499,900 \\
\hline $\begin{array}{l}\text { TEXAS EASTERN TRANSMISSION CORPORATION } \\
\text { (PROGAS LIMITED) } \\
\text { AVG Daily Quantity } \\
\text { Total Price (Niagara Falls, New York) } \\
\text { Commodity Component } \\
\text { Demand Component } \\
\text { Reservation Fee Component }\end{array}$ & 29.0 & $\begin{array}{r}0.0 \\
0.00 \\
0.00 \\
0.00 \\
0.00\end{array}$ & $\begin{array}{r}0.0 \\
0.00 \\
0.00 \\
0.00 \\
0.00\end{array}$ & $\begin{array}{r}3.2 \\
6.90 \\
2.03 \\
4.87 \\
0.00\end{array}$ & $\begin{array}{r}0.0 \\
0.00 \\
0.00 \\
0.00 \\
0.00\end{array}$ & $\begin{array}{r}0.0 \\
0.00 \\
0.00 \\
0.00 \\
0.00\end{array}$ & $\begin{array}{r}0.2 \\
2.65 \\
2.65 \\
0.00 \\
0.00\end{array}$ & $\begin{array}{l}0.0 \\
0.00 \\
0.00 \\
0.00 \\
0.00\end{array}$ & $\begin{array}{r}0.0 \\
0.00 \\
0.00 \\
0.00 \\
0.00\end{array}$ & $\begin{array}{r}0.0 \\
0.00 \\
0.00 \\
0.00 \\
0.00\end{array}$ & $\begin{array}{r}0.0 \\
0.00 \\
0.00 \\
0.00 \\
0.00\end{array}$ & $\begin{array}{r}0.0 \\
0.00 \\
0.00 \\
0.00 \\
0.00\end{array}$ & $\begin{array}{r}0.0 \\
0.00 \\
0.00 \\
0.00 \\
0.00\end{array}$ & 0 & 107,443 \\
\hline $\begin{array}{l}\text { THE MONTANA POWER COMPANY } \\
\text { (CANADIAN-MONTANA PIPELINE COMPANY) } \\
\text { AVG Daily Quant ity } \\
\text { Total Price (Whitlash, Montana) } \\
\text { Commodity Component } \\
\text { Demand Component } \\
\text { Reservation Fee Component }\end{array}$ & 50.0 & $\begin{array}{l}26.5 \\
1.53 \\
1.53 \\
0.00 \\
0.00\end{array}$ & $\begin{array}{l}25.7 \\
1.55 \\
1.55 \\
0.00 \\
0.00\end{array}$ & $\begin{array}{l}24.6 \\
1.56 \\
1.56 \\
0.00 \\
0.00\end{array}$ & $\begin{array}{l}19.2 \\
1.55 \\
1.55 \\
0.00 \\
0.00\end{array}$ & $\begin{array}{l}10.7 \\
1.54 \\
1.54 \\
0.00 \\
0.00\end{array}$ & $\begin{array}{r}8.3 \\
1.53 \\
1.53 \\
0.00 \\
0.00\end{array}$ & $\begin{array}{l}11.5 \\
1.52 \\
1.52 \\
0.00 \\
0.00\end{array}$ & $\begin{array}{l}14.0 \\
1.49 \\
1.49 \\
0.00 \\
0.00\end{array}$ & $\begin{array}{l}14.9 \\
1.48 \\
1.48 \\
0.00 \\
0.00\end{array}$ & $\begin{array}{l}21.2 \\
1.47 \\
1.47 \\
0.00 \\
0.00\end{array}$ & $\begin{array}{l}21.3 \\
1.48 \\
1.48 \\
0.00 \\
0.00\end{array}$ & $\begin{array}{l}22.8 \\
1.47 \\
1.47 \\
0.00 \\
0.00\end{array}$ & $2,001,226$ & $6,721,889$ \\
\hline $\begin{array}{l}\text { TM STAR FUEL COMPANY } \\
\text { (CANWEST GAS SUPPLY) } \\
\text { AVG Daily Quantity } \\
\text { Total Price (SUmas, washington) } \\
\text { Commodity Component } \\
\text { Demand Comporient } \\
\text { Reservation Fee Component }\end{array}$ & 10.0 & $\begin{array}{r}0.0 \\
0.00 \\
0.00 \\
0.00 \\
0.00\end{array}$ & $\begin{array}{r}0.0 \\
0.00 \\
0.00 \\
0.00 \\
0.00\end{array}$ & $\begin{array}{r}0.0 \\
0.00 \\
0.00 \\
0.00 \\
0.00\end{array}$ & $\begin{array}{r}0.0 \\
0.00 \\
0.00 \\
0.00 \\
0.00\end{array}$ & $\begin{array}{l}0.0 \\
0.00 \\
0.00 \\
0.00 \\
0.00\end{array}$ & $\begin{array}{r}0.0 \\
0.00 \\
0.00 \\
0.00 \\
0.00\end{array}$ & $\begin{array}{r}0.0 \\
0.00 \\
0.00 \\
0.00 \\
0.00\end{array}$ & $\begin{array}{r}0.0 \\
0.00 \\
0.00 \\
0.00 \\
0.00\end{array}$ & $\begin{array}{r}0.0 \\
0.00 \\
0.00 \\
0.00 \\
0.00\end{array}$ & $\begin{array}{r}0.0 \\
0.00 \\
0.00 \\
0.00 \\
0.00\end{array}$ & $\begin{array}{r}8.6 \\
1.83 \\
1.83 \\
0.00 \\
0.00\end{array}$ & $\begin{array}{r}9.1 \\
1.83 \\
1.83 \\
0.00 \\
0.00\end{array}$ & 542,810 & 542,810 \\
\hline $\begin{array}{l}\text { TRANSCO ENERGY MARKETING COMPANY ( } 3 \text { ) } \\
\text { (CANSTATES GAS MARKETING) } \\
\text { AVG Daily Uuantity } \\
\text { Total Price (Niagara Falls, New York) } \\
\text { Commodity Component } \\
\text { Demand Component } \\
\text { Reservation Fee Component }\end{array}$ & 48.4 & $\begin{array}{l}48.6 \\
2.85 \\
1.98 \\
0.87 \\
0.00\end{array}$ & $\begin{array}{l}48.5 \\
2.79 \\
1.83 \\
0.96 \\
0.00\end{array}$ & $\begin{array}{l}48.4 \\
2.30 \\
1.43 \\
0.87 \\
0.00\end{array}$ & $\begin{array}{l}48.5 \\
2.34 \\
1.45 \\
0.89 \\
0.00\end{array}$ & $\begin{array}{l}48.4 \\
2.64 \\
1.76 \\
0.88 \\
0.00\end{array}$ & $\begin{array}{l}31.6 \\
3.57 \\
2.20 \\
1.37 \\
0.00\end{array}$ & $\begin{array}{l}19.2 \\
2.89 \\
1.69 \\
1.20 \\
0.00\end{array}$ & $\begin{array}{l}48.5 \\
2.42 \\
1.57 \\
0.85 \\
0.00\end{array}$ & $\begin{array}{l}48.4 \\
2.52 \\
1.66 \\
0.86 \\
0.00\end{array}$ & $\begin{array}{l}48.5 \\
2.78 \\
1.95 \\
0.83 \\
0.00\end{array}$ & $\begin{array}{l}48.4 \\
2.55 \\
1.67 \\
0.88 \\
0.00\end{array}$ & $\begin{array}{l}48.5 \\
2.63 \\
1.79 \\
0.84 \\
0.00\end{array}$ & $4,457,963$ & $16,316,862$ \\
\hline
\end{tabular}

(1) Texas Eastern's contracts expired on April 1, 1993 as part of its overall restructuring under FERC Order 636.

(2) Texas Eastern's contracts expired on April 1, 1993 as part of its overall restructuring under FERC Order 636. waddington, New York at the above listed prices. 
VOLUME AND PRICE REPORT

Long Term Imports

Long Term Imports
Volumes (MMCf/d) \& Prices ( $\$$ /MMBTU) of Natural

Volumes (MMCf/d) \& Prices (\$/MMBTU) of Natural
Gas Imported During the Past 12 Months $01 / 01 / 93^{-} 12 / 31 / 93$

\section{Long-Term Importer}

TRANSCO ENERGY MARKETING COMPANY-BG\&E (CANSTATES GAS MARKETING)

AVG Daily Quantity

Total Price (Niagara Falls, New York) Commodity Component

Demand Component

Reservation fee Component

Auth. $1993 \quad 1993 \quad 1993 \quad 1993 \quad 1993$

25.0

$25.0 \quad 24$

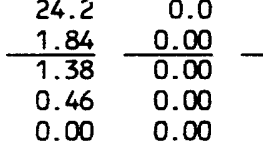

Mar. Apr.

1993
May

1993

1993

1993

1993
$\quad$ Sep.

1993
Oct.

Oct. Nov.

Dec.

$0.0 \quad 0.0$

$\begin{array}{lll}0.0 & 0.0 \quad 3.9\end{array}$

.9

$0.00 \quad 0.00$

$\frac{0.00}{0.00} \frac{0.00}{0.00}$

$\frac{0.00}{0.00}-\frac{0.00}{0.00}$

$\frac{1.42}{1.03}$

0.00

22.8
1.57

$\begin{array}{lll}0.0 & 14.1 & 24.0\end{array}$ $0.00 \frac{1.65}{1.24}-\frac{1.73}{1.26}$

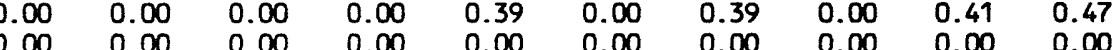

TRANSCO ENERGY MARKETING COMPANY-LILCO (CANSTATES GAS MARKETING)

AVG Daily Quantity

Total Price (Niagara Falls, New York

Commodity Component

Demand Component

Reservation Fee Component

15

\begin{tabular}{|c|c|c|c|c|c|c|c|c|c|c|c|}
\hline $\begin{array}{r}0.0 \\
0.00 \\
0.00 \\
0.00 \\
0.00\end{array}$ & $\begin{array}{l}1.6 \\
1.41 \\
1.02 \\
0.39 \\
0.00\end{array}$ & $\begin{array}{r}7.6 \\
1.40 \\
1.05 \\
0.35 \\
0.00\end{array}$ & $\begin{array}{r}0.0 \\
0.00 \\
0.00 \\
0.00 \\
0.00\end{array}$ & $\begin{array}{r}0.0 \\
0.00 \\
0.00 \\
0.00 \\
0.00\end{array}$ & $\begin{array}{l}14.2 \\
1.77 \\
1.25 \\
0.52 \\
0.00\end{array}$ & $\begin{array}{r}0.0 \\
0.00 \\
0.00 \\
0.00 \\
0.00\end{array}$ & $\begin{array}{r}0.0 \\
0.00 \\
0.00 \\
0.00 \\
0.00\end{array}$ & $\begin{array}{r}0.0 \\
0.00 \\
0.00 \\
0.00 \\
0.00\end{array}$ & $\begin{array}{r}0.0 \\
0.00 \\
0.00 \\
0.00 \\
0.00\end{array}$ & $\begin{array}{l}0.0 \\
0.00 \\
0.00 \\
0.00 \\
0.00\end{array}$ & $\begin{array}{r}1.5 \\
1.60 \\
1.17 \\
0.43 \\
0.00\end{array}$ \\
\hline
\end{tabular}

TRANSCO ENERGY MARKETING COMPANY-PSE\&G

(CANSTATES GAS MARKETING)

AVG Daily Quantity

Total Price (Niagara Falls, New York)

Commodity Component

Reservation Fee Component

VALLEY GAS COMPANY

(ALBERTA NORTHEAST GAS (WGM LTD. 1))

AVG Daily Quantity

Commodity Component

Demand Component

Reservation Fee Component

\begin{tabular}{llllllllllllll}
.0 & 23.3 & 0.0 & 18.7 & 23.4 & 9.5 & 20.3 & 0.0 & 0.0 & 32.4 & 0.0 & 24.5 & 0.0 \\
1.40 & 0.00 & 1.14 & 1.19 & 1.53 & 1.49 & 0.00 & 0.00 & $\frac{1.49}{1.00}$ & 0.00 & $\frac{1.54}{1.02}$ & 0.00 \\
\hline 1.12 & 0.00 & 0.96 & 0.98 & 1.26 & 1.16 & 0.00 & 0.00 & 1.23 & 0.00 & 1.27 & 0.00 \\
0.28 & 0.00 & 0.18 & 0.21 & 0.27 & 0.33 & 0.00 & 0.00 & 0.26 & 0.00 & 0.27 & 0.00 \\
0.00 & 0.00 & 0.00 & 0.00 & 0.00 & 0.00 & 0.00 & 0.00 & 0.00 & 0.00 & 0.00 & 0.00
\end{tabular}

1.0

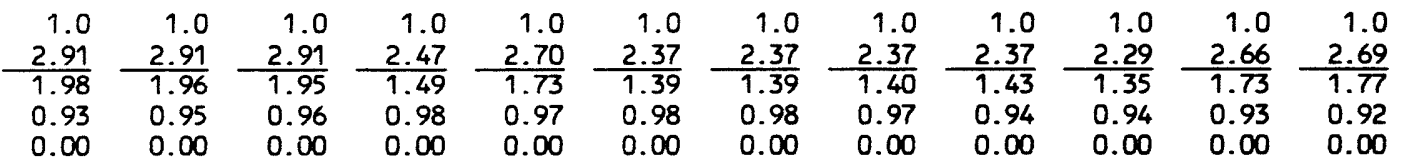

VERMONT GAS SYSTEMS INC

(WGM LTD.)

AVG Daily Quantity

Total Price (Highgate Springs, Vermont)

Commodity Component

Demand Component

Reservation Fee Comporient

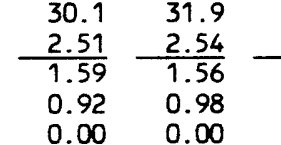

$0.00 \quad 0.98$

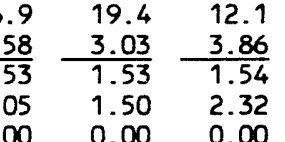

$0.00 \quad 0.00$
$11.8 \quad 12.0$

$\frac{4.00}{1.54}$

2.46
46,326

754,146

$4,612,786$

90,707

360,058

$7,090,865$ 


\section{VOLUME AND PRICE REPORT \\ Long Term Imports}

Volumes (MMCf/d) \& Prices ( $\$$ /MMBTU) of Natural

Gas Imported During the Past 12 Months $01 / 01 / 93-12 / 31 / 93$

\section{Long-Term Importer}

WASHINGTON NATURAL GAS COMPANY (AMOCO CANADA)

AVG Daily Quantity

Total Price (Sumas, Washington) Commodity Component

Demand Component

Reservation Fee Component

WASHINGTON NATURAL GAS COMPANY

(MOBIL OIL CANADA)

AVG Daily Quantity

Total Price (Sumas, washington)

Commodity Component

Demand Component

Reservation Fee Component

WASHINGTON NATURAL GAS COMPANY

(POCO PETROLEUMS LTD.)

AVG Daily Quantity

Total Price (Sumas, Washington)

Commodity Componen

Demand Component

Reservation Fee Component

WASHINGTON NATURAL GAS COMPANY

(CANADIAN HYDROCARBONS MARKETING)

AVG Daily Quantity

Total Price (Sumas, Washington)

Commodity Component

Demand Component

Reservation Fee Component

WASHINGTON NATURAL GAS COMPANY

(CANADIAN HYDROCARBONS MARKETING)

AVG Daily Quantity

Total Price (Eastport, Idaho)

Commodity Component

Reservation Fee Component
Auth
vo!s 24

\begin{tabular}{rrrrrrrrrrrrrr}
24.3 & 22.3 & 24.2 & 24.1 & 24.2 & 24.2 & 23.9 & 22.7 & 23.4 & 24.2 & 24.2 & 24.2 \\
1.72 & 1.83 & 1.74 & $\frac{1.57}{1.5}$ & 1.54 & 1.54 & 1.53 & 1.52 & 1.53 & 1.49 & $\frac{2.15}{2.15}$ \\
\hline 1.31 & 1.32 & 1.32 & 1.14 & 1.13 & 1.12 & 1.10 & 1.08 & 1.07 & 1.06 & 1.75 & 1.75 \\
0.41 & 0.51 & 0.42 & 0.43 & 0.41 & 0.42 & 0.43 & 0.44 & 0.46 & 0.43 & 0.40 & 0.40 \\
0.00 & 0.00 & 0.00 & 0.00 & 0.00 & 0.00 & 0.00 & 0.00 & 0.00 & 0.00 & 0.00 & 0.00
\end{tabular}

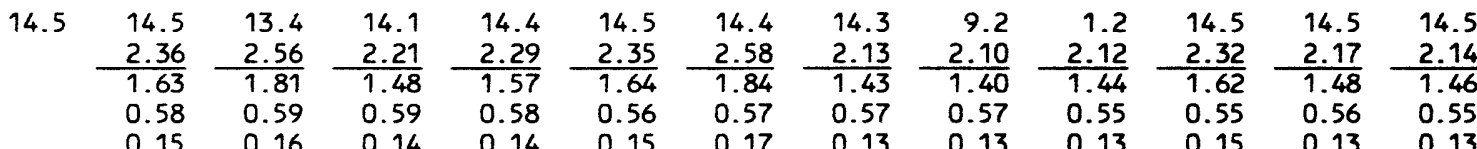

\begin{tabular}{rrrrrrrrrrrrrr}
14.4 & 13.1 & 14.5 & 14.5 & 14.5 & 14.5 & 0.0 & 9.5 & 13.2 & 12.2 & 14.5 & 14.5 & 14.5 \\
& 1.80 & 1.87 & 1.82 & 1.84 & 2.10 & 0.00 & 2.25 & 2.12 & 2.30 & 2.18 & 2.29 & 2.28 \\
\cline { 2 - 6 } & 1.35 & 1.35 & 1.35 & 1.35 & 1.64 & 0.00 & 1.55 & 1.65 & 1.92 & 1.75 & 1.84 & 1.84 \\
0.45 & 0.52 & 0.47 & 0.49 & 0.46 & 0.00 & 0.70 & 0.47 & 0.38 & 0.43 & 0.45 & 0.44 \\
0.00 & 0.00 & 0.00 & 0.00 & 0.00 & 0.00 & 0.00 & 0.00 & 0.00 & 0.00 & 0.00 & 0.00
\end{tabular}

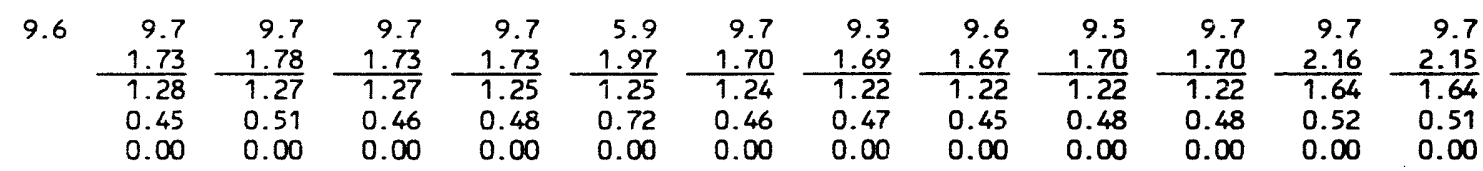

$889,277 \quad 3,406,360$

\begin{tabular}{cccccccccccc}
0.0 & 0.0 & 0.0 & 0.0 & 0.0 & 0.0 & 0.0 & 0.0 & 0.0 & 0.0 & 47.7 & 33.9 \\
0.00 & 0.00 & 0.00 & 0.00 & $\frac{0.00}{0.00}$ & $\frac{0.00}{0.00}$ & $\frac{0.00}{0.00}$ & $\frac{0.00}{0.00}$ & $\frac{2.26}{2.81}$ \\
\hline 0.00 & 0.00 & 0.00 & 0.00 & 0.00 & 0.00 & 0.00 & 0.00 & 0.00 & 0.00 & $\frac{1.82}{2.41}$ & $\frac{1.00}{0.00}$ \\
0.00 & 0.00 & 0.00 & 0.00 & 0.00 & 0.00 & 0.00 & 0.00 & 0.00 & 0.44 & 0.40 \\
0.00 & 0.00 & 0.00 & 0.00 & 0.00 & 0.00 & 0.00 & 0.00 & 0.00 & 0.00 & 0.00 & 0.00
\end{tabular}




\section{VOLUNE AND PRICE REPORT}

Volumes (MMcflding Term Imports

Gas Imported During the Past 12 Months $01 / 01 / 93^{-} 12 / 31 / 93$

\section{Long-Term Importer}

WASHINGTON WATER POWER COMPANY (CANADIAN HYDROCARBONS MARKETING) AVG Daily Quantity

Total Price (Eastport, Idaho)

Commodi ty Component

Demand Component

Reservation Fee Component

WASHINGTON WATER POWER COMPANY

(AEC OIL \& GAS COMPANY)

AVG Daily Quantity

Total Price (Eastport, Idaho)

Cominodity Component

Demand Component

Reservation Fee Component

WASHINGTON WATER POWER COMPANY

(AMERADA HESS CANADA LTD.)

AVG Daily Quantity

Total Price (Eastport, Idaho)

Commodity Component

Demand Component

Reservation Fee Component

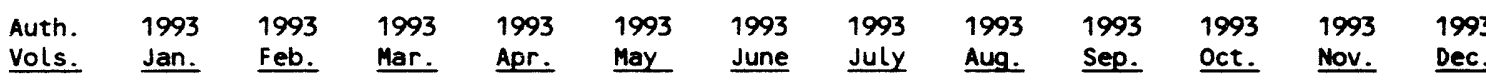

19.
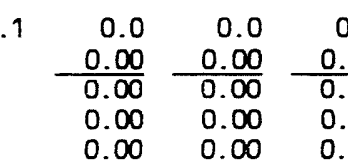

25.6

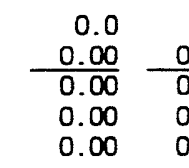

\begin{tabular}{lr}
0.0 & 0.0 \\
.00 & 0.00 \\
\hline 00 & 0.00 \\
.00 & 0.00 \\
.00 & 0.00
\end{tabular}
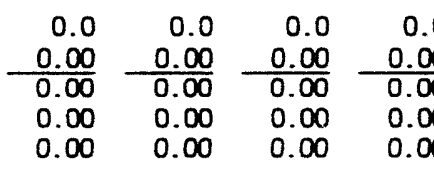

\begin{tabular}{ll}
.0 & 0.0 \\
.00 & 0.00 \\
\hline .00 & 0.00 \\
.00 & 0.00
\end{tabular}

\begin{tabular}{|c|c|c|}
\hline $\begin{array}{r}0.0 \\
0.00 \\
0.00\end{array}$ & $\begin{array}{l}0.0 \\
0.00 \\
0.00 \\
0.00 \\
0.00\end{array}$ & $\begin{array}{r}1.2 \\
2.14 \\
1.81 \\
0.33 \\
0.00\end{array}$ \\
\hline
\end{tabular}

63,898

63,898

\begin{tabular}{lrrrr}
0.0 & 0.0 & 0.0 & 0.0 & 0.0 \\
0.00 & 0.00 & 0.00 & 0.00 & 0.00 \\
\cline { 3 - 4 } & 0.00 & 0.00 & 0.00 & 0.00 \\
0.00 & 0.00 & 0.00 & 0.00 & 0.00 \\
0.00 & 0.00 & 0.00 & 0.00 & 0.00
\end{tabular}

\begin{tabular}{lrrrr}
0.0 & 0.0 & 0.0 & 15.3 & 15.5 \\
0.00 & 0.00 & 0.00 & 2.30 & 2.29 \\
\cline { 5 - 5 } & 0.00 & 0.00 & 2.22 & 2.22 \\
0.00 & 0.00 & 0.00 & 0.08 & 0.07 \\
0.00 & 0.00 & 0.00 & 0.00 & 0.00
\end{tabular}

938,724

938,724

WASHINGTON WATER POWER COMPANY

(PANCANADIAN PETROLEUM LIMITED)

AVG Daily Quantity

Total Price (Eastport, Idaho)

Commodity Component

Demand Component

Reservation Fee Component

WESTERN GAS MARKETING INC. (1)

(WGM LTO.)

AVG Daily Quantity

Total Price (Noyes, Minnesota)

Component

Reservation Fee Component
19.0

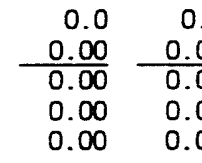

16.8

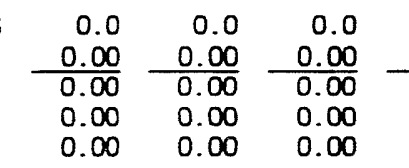

\begin{tabular}{rrrrrrrrrr}
0.0 & 0.0 & 0.0 & 0.0 & 0.0 & 0.0 & 0.0 & 10.4 & 15.5 \\
0.00 & 0.00 & 0.00 & 0.00 & $\frac{0.00}{0.00}$ & $\frac{0.00}{0.00}$ & $\frac{0.00}{1.87}$ & $\frac{1.29}{1.00}$ \\
\cline { 2 - 8 } & 0.00 & 0.00 & 0.00 & 0.00 & 0.00 & 0.00 & 1.42 & 2.22 \\
0.00 & 0.00 & 0.00 & 0.00 & 0.00 & 0.00 & 0.00 & 0.45 & 0.07 \\
0.00 & 0.00 & 0.00 & 0.00 & 0.00 & 0.00 & 0.00 & 0.00 & 0.00
\end{tabular}

794,580

25.0

\begin{tabular}{rrrrrrrrrrrrr}
7.9 & 3.5 & 11.3 & 0.9 & 0.0 & 7.1 & 25.0 & 17.8 & 6.5 & 7.3 & 21.3 & 18.6 \\
3.50 & 5.95 & 2.64 & 18.04 & 0.00 & 3.53 & 2.35 & 2.62 & 4.39 & 3.83 & 2.37 & 2.58 \\
\hline 1.59 & 1.42 & 1.37 & 2.01 & 0.00 & 1.71 & 1.71 & 1.75 & 2.05 & 1.72 & $\frac{1.75}{1.91}$ \\
1.91 & 4.53 & 1.27 & 16.03 & 0.00 & 1.82 & 0.64 & 0.87 & 2.34 & 2.11 & 0.62 & 0.67 \\
0.00 & 0.00 & 0.00 & 0.00 & 0.00 & 0.00 & 0.00 & 0.00 & 0.00 & 0.00 & 0.00 & 0.00
\end{tabular}

(1) Volumes are resold to Northern Natural; the point of entry varies seasonally -- for the most part, volumes are delivered to Carlton, Minnesota via Noyes, MN or St. Clair, MI (via backhaul) in the winter, and to Port of Morgan in the summer. 


\section{VOLULA AND PRICE REPORT}

Iong Term Imports

Volumes (MMCf/d) \& Prices (S/MMBTU) of Natural

Gas Imported During the Past 12 Months $01 / 01 / 93^{-} 12 / 31 / 93$

\section{Long-Term Importer}

WISCONSIN FUEL \& LIGHT COMPANY (PROGAS LIMITED)

AVG Caily Quantity

Total Price (Noyes, Minnesota) Commodity Component

Demand Component

Reservation Fee Component

UISCONSIN FUEL \& LIGHT COMPANY

(WGM LTD.)

Total Price (Noyes, Minnesota) Comuodi ty Component

Deservation Fee

component

WISCONSIN NATURAL GAS COMPANY

(PROGAS LIMITED)

AVG Daily Quantity

Total Price (Noyes, Minnesota) Commodity Component

Demand Component

Reservation fee Component

Vols.

1993
Mar.

19931993

Aug.

uly Ang

\begin{tabular}{ccccccccccccc}
0 & 0.0 & 0.0 & 0.0 & 0.0 & 0.0 & 0.0 & 0.0 & 0.0 & 0.0 & 0.0 & 2.8 & 3.0 \\
0.00 & 0.00 & 0.00 & 0.00 & 0.00 & 0.00 & 0.00 & 0.00 & 0.00 & 0.00 & 2.25 & $\frac{2.52}{2.00}$ \\
\hline 0.00 & 0.00 & 0.00 & 0.00 & 0.00 & 0.00 & 0.00 & 0.00 & 0.00 & 0.00 & 1.70 & 2.00 \\
0.00 & 0.00 & 0.00 & 0.00 & 0.00 & 0.00 & 0.00 & 0.00 & 0.00 & 0.00 & 0.50 & 0.4. \\
0.00 & 0.00 & 0.00 & 0.00 & 0.00 & 0.00 & 0.00 & 0.00 & 0.00 & 0.00 & 0.05 & 0.05
\end{tabular}

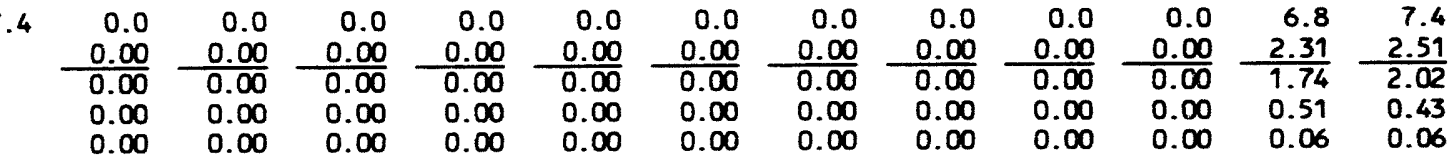

\begin{tabular}{lllll}
0.0 & 0.0 & 0.0 & 0.0 & 0.0 \\
0.00 & 0.00 & 0.00 & 0.00 & 0.00 \\
\cline { 3 - 5 } & 0.00 & 0.00 & 0.00 & 0.00 \\
0.00 & 0.00 & 0.00 & 0.00 & 0.00 \\
0.00 & 0.00 & 0.00 & 0.00 & 0.00
\end{tabular}

WISCONSIN NATURAL GAS COMPANY

(WGM LTD.)

AVG Daily Quantity

Total Price (Noyes, Minnesota)

Reservation fee Component

WISCONSIN POWER \& LIGHT COMPANY

(WGM LTD.)

AVG Daily Quantity

Total Price (Noyes, Minnesota)

Comodity Component

Reservation Fee Component

\begin{tabular}{|c|c|c|c|c|c|c|c|c|c|c|}
\hline $\begin{array}{l}0.0 \\
0.00 \\
0.00 \\
0.00 \\
0.00\end{array}$ & $\begin{array}{r}0.0 \\
0.00 \\
0.00 \\
0.00 \\
0.00\end{array}$ & $\begin{array}{r}0.0 \\
0.00 \\
0.00 \\
0.00 \\
0.00\end{array}$ & $\begin{array}{r}0.0 \\
0.00 \\
0.00 \\
0.00 \\
0.00\end{array}$ & $\begin{array}{r}0.0 \\
0.00 \\
0.00 \\
0.00 \\
0.00\end{array}$ & $\begin{array}{r}0.0 \\
0.00 \\
0.00 \\
0.00 \\
0.00\end{array}$ & $\begin{array}{r}0.0 \\
0.00 \\
0.00 \\
0.00 \\
0.00\end{array}$ & $\begin{array}{r}0.0 \\
0.00 \\
0.00 \\
0.00 \\
0.00\end{array}$ & $\begin{array}{r}0.0 \\
0.00 \\
0.00 \\
0.00 \\
0.00\end{array}$ & $\begin{array}{r}0.0 \\
0.00 \\
0.00 \\
0.00 \\
0.00\end{array}$ & $\begin{array}{r}18.1 \\
2.48 \\
1.72 \\
0.69 \\
0.07\end{array}$ \\
\hline
\end{tabular}

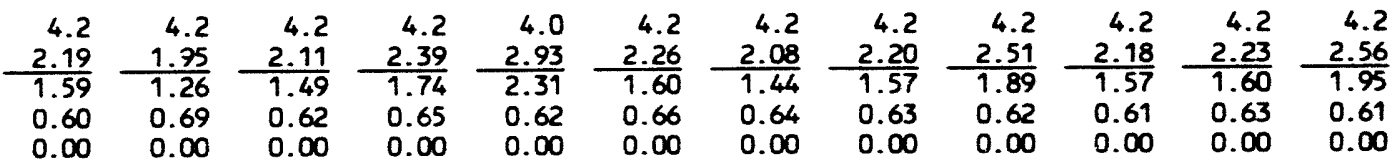

433,214

433,214

$177,253 \quad 177,253$

$566,994 \quad 566,994$

$1,093,355 \quad 1,093,355$

$388,617 \quad 1,540,429$ 


\section{VOLUTE AMD PRICE REPORT}

\section{Long Term Inporte}

Volumes (MMcf/d) \& Prices (S/MMBTU) of Natural

Gas Imported During the Past 12 Months $01 / 01 / 93-12 / 31 / 93$

\section{Long-Tern Importer}

WISCONSIN POWER \& LIGHT COMPANY (PROGAS LIMITED)

AVG Daily Quantity

Total Price (Noyes, Minnesota) Comeodity Component

Demand Component

Reservation Fee Component

WISCONSIN PONER \& LIGHT COMPANY (WGH LTD.)

AVG Daily Quantity

Total Price (Noyes, Minnesota)

Commodity Component

Demand Component

Reservation Fee Component

WISCONSIN PUBLIC SERVICE CORPORATION (PROGAS LIMITED)

AVG Daily Quantity

Total Price (Noyes, Minnesota)

Commodity Component

Demand Component

Reservation Fee Component

WISCONSIN PUBLIC SERVICE CORPORATION (WGM LTD.)

AVG Daily Quantity

Total Price (Noyes, Minnesota)

Commodity Component

Demand Component

Reservation fee component

YANKEE GAS SERVICES CO.

(ALBERTA MORTHEAST GAS (HGM LTD. 1))

AVG Daily Quantity

Total Price (Waddington, New York)

Commodity Component

Demand Component

Reservation Fee Component

\begin{tabular}{|c|c|c|c|c|c|c|c|c|c|c|c|c|c|c|}
\hline $\begin{array}{l}\text { Auth. } \\
\text { Vols. }\end{array}$ & $\begin{array}{l}1993 \\
\text { Jan. }\end{array}$ & $\begin{array}{l}1993 \\
\text { Feb. }\end{array}$ & $\begin{array}{l}1993 \\
\text { Mar. }\end{array}$ & $\begin{array}{l}1993 \\
\text { Apr. }\end{array}$ & $\begin{array}{l}1993 \\
\text { may }\end{array}$ & $\begin{array}{l}1993 \\
\text { June } \\
\end{array}$ & $\begin{array}{l}1993 \\
\text { July } \\
\end{array}$ & $\begin{array}{l}1993 \\
\text { Aug. }\end{array}$ & $\begin{array}{l}1993 \\
\text { Sep. }\end{array}$ & $\begin{array}{l}1993 \\
\text { Oct. }\end{array}$ & $\begin{array}{l}1993 \\
\text { Nov. }\end{array}$ & $\begin{array}{l}1993 \\
\text { Dec. }\end{array}$ & $\begin{array}{c}4093 \\
\text { TOTAL MCf }\end{array}$ & $\begin{array}{c}\text { YTD } \\
\text { TOTAL nCf } \\
\end{array}$ \\
\hline 3.4 & $\begin{array}{l}0.0 \\
0.00 \\
0.00 \\
0.00 \\
0.00\end{array}$ & $\begin{array}{l}0.0 \\
0.00 \\
0.00 \\
0.00 \\
0.00\end{array}$ & $\begin{array}{r}0.0 \\
0.00 \\
0.00 \\
0.00 \\
0.00\end{array}$ & $\begin{array}{l}0.0 \\
0.00 \\
0.00 \\
0.00 \\
0.00\end{array}$ & $\begin{array}{r}0.0 \\
0.00 \\
0.00 \\
0.00 \\
0.00\end{array}$ & $\begin{array}{r}0.0 \\
0.00 \\
0.00 \\
0.00 \\
0.00\end{array}$ & $\begin{array}{r}0.0 \\
0.00 \\
0.00 \\
0.00 \\
0.00\end{array}$ & $\begin{array}{r}0.0 \\
0.00 \\
0.00 \\
0.00 \\
0.00\end{array}$ & $\begin{array}{r}0.0 \\
0.00 \\
0.00 \\
0.00 \\
0.00\end{array}$ & $\begin{array}{l}0.0 \\
0.00 \\
0.00 \\
0.00 \\
0.00\end{array}$ & $\begin{array}{r}3.3 \\
2.20 \\
1.69 \\
0.51 \\
0.00\end{array}$ & $\begin{array}{r}3.3 \\
2.45 \\
1.97 \\
0.48 \\
0.00\end{array}$ & 202,292 & 202,292 \\
\hline 8.4 & $\begin{array}{r}0.0 \\
0.00 \\
0.00 \\
0.00 \\
0.00\end{array}$ & $\begin{array}{r}0.0 \\
0.00 \\
0.00 \\
0.00 \\
0.00\end{array}$ & $\begin{array}{r}0.0 \\
0.00 \\
0.00 \\
0.00 \\
0.00\end{array}$ & $\begin{array}{r}0.0 \\
0.00 \\
0.00 \\
0.00 \\
0.00\end{array}$ & $\begin{array}{l}0.0 \\
0.00 \\
0.00 \\
0.00 \\
0.00\end{array}$ & $\begin{array}{r}0.0 \\
0.00 \\
0.00 \\
0.00 \\
0.00\end{array}$ & $\begin{array}{r}0.0 \\
0.00 \\
0.00 \\
0.00 \\
0.00\end{array}$ & $\begin{array}{r}0.0 \\
0.00 \\
0.00 \\
0.00 \\
0.00\end{array}$ & $\begin{array}{r}0.0 \\
0.00 \\
0.00 \\
0.00 \\
0.00\end{array}$ & $\begin{array}{r}0.0 \\
0.00 \\
0.00 \\
0.00 \\
0.00\end{array}$ & $\begin{array}{r}8.3 \\
2.20 \\
1.72 \\
0.48 \\
0.00\end{array}$ & $\begin{array}{r}8.2 \\
2.40 \\
1.96 \\
0.44 \\
0.00\end{array}$ & 501,686 & 501,686 \\
\hline 11.1 & $\begin{array}{r}0.0 \\
0.00 \\
0.00 \\
0.00 \\
0.00\end{array}$ & $\begin{array}{r}0.0 \\
0.00 \\
0.00 \\
0.00 \\
0.00\end{array}$ & $\begin{array}{r}0.0 \\
0.00 \\
0.00 \\
0.00 \\
0.00\end{array}$ & $\begin{array}{r}0.0 \\
0.00 \\
0.00 \\
0.00 \\
0.00\end{array}$ & $\begin{array}{r}0.0 \\
0.00 \\
0.00 \\
0.00 \\
0.00\end{array}$ & $\begin{array}{r}0.0 \\
0.00 \\
0.00 \\
0.00 \\
0.00\end{array}$ & $\begin{array}{r}0.0 \\
0.00 \\
0.00 \\
0.00 \\
0.00\end{array}$ & $\begin{array}{r}0.0 \\
0.00 \\
0.00 \\
0.00 \\
0.00\end{array}$ & $\begin{array}{r}0.0 \\
0.00 \\
0.00 \\
0.00 \\
0.00\end{array}$ & $\begin{array}{r}0.0 \\
0.00 \\
0.00 \\
0.00 \\
0.00\end{array}$ & $\begin{array}{l}11.1 \\
2.21 \\
1.69 \\
0.48 \\
0.04\end{array}$ & $\begin{array}{l}11.1 \\
2.49 \\
1.97 \\
0.47 \\
0.05\end{array}$ & 676,275 & 676,275 \\
\hline 27.4 & $\begin{array}{r}0.0 \\
0.00 \\
0.00 \\
0.00 \\
0.00\end{array}$ & $\begin{array}{r}0.0 \\
0.00 \\
0.00 \\
0.00 \\
0.00\end{array}$ & $\begin{array}{r}0.0 \\
0.00 \\
0.00 \\
0.00 \\
0.00\end{array}$ & $\begin{array}{r}0.0 \\
0.00 \\
0.00 \\
0.00 \\
0.00\end{array}$ & $\begin{array}{r}0.0 \\
0.00 \\
0.00 \\
0.00 \\
0.00\end{array}$ & $\begin{array}{r}0.0 \\
0.00 \\
0.00 \\
0.00 \\
0.00\end{array}$ & $\begin{array}{r}0.0 \\
0.00 \\
0.00 \\
0.00 \\
0.00\end{array}$ & $\begin{array}{r}0.0 \\
0.00 \\
0.00 \\
0.00 \\
0.00\end{array}$ & $\begin{array}{r}0.0 \\
0.00 \\
0.00 \\
0.00 \\
0.00\end{array}$ & $\begin{array}{r}0.0 \\
0.00 \\
0.00 \\
0.00 \\
0.00\end{array}$ & $\begin{array}{l}27.2 \\
2.24 \\
1.72 \\
0.47 \\
0.05\end{array}$ & $\begin{array}{l}27.3 \\
2.45 \\
1.96 \\
0.43 \\
0.06\end{array}$ & $1,662,679$ & $1,662,679$ \\
\hline 28.7 & $\begin{array}{l}28.7 \\
2.91 \\
1.98 \\
0.93 \\
0.00\end{array}$ & $\begin{array}{l}28.7 \\
2.91 \\
1.96 \\
0.95 \\
0.00\end{array}$ & $\begin{array}{l}28.7 \\
2.91 \\
1.95 \\
0.96 \\
0.00\end{array}$ & $\begin{array}{l}28.7 \\
2.47 \\
1.49 \\
0.98 \\
0.00\end{array}$ & $\begin{array}{l}28.7 \\
2.70 \\
1.73 \\
0.97 \\
0.00\end{array}$ & $\begin{array}{l}28.7 \\
2.37 \\
1.39 \\
0.98 \\
0.00\end{array}$ & $\begin{array}{l}28.7 \\
2.37 \\
1.39 \\
0.98 \\
0.00\end{array}$ & $\begin{array}{l}28.7 \\
2.37 \\
1.40 \\
0.97 \\
0.00\end{array}$ & $\begin{array}{l}28.7 \\
2.37 \\
1.43 \\
0.94 \\
0.00\end{array}$ & $\begin{array}{l}28.7 \\
2.29 \\
1.35 \\
0.94 \\
0.00\end{array}$ & $\begin{array}{l}28.7 \\
2.66 \\
1.73 \\
0.93 \\
0.00\end{array}$ & $\begin{array}{l}28.7 \\
2.69 \\
1.77 \\
0.92 \\
0.00\end{array}$ & $2,640,400$ & $10,504,767$ \\
\hline
\end{tabular}




\section{VOIUNIS NID PRICE REPORT}

Iong Tere Imports

Volumes (MMCf/d) \& Prices (S/MMBTU) of Natural

Gas Imported During the Past 12 Months 01/01/93 - 12/31/93

\begin{tabular}{|c|c|c|c|c|c|c|c|c|c|c|c|c|c|c|c|}
\hline Long-Term Importer & $\begin{array}{l}\text { Auth. } \\
\text { Vols. }\end{array}$ & $\begin{array}{l}1993 \\
\text { Jan. } \\
\end{array}$ & $\begin{array}{l}1993 \\
\text { Feb. }\end{array}$ & $\begin{array}{l}1993 \\
\text { Mar. }\end{array}$ & $\begin{array}{l}1993 \\
\text { Apr. }\end{array}$ & $\begin{array}{l}1993 \\
\text { May }\end{array}$ & $\begin{array}{l}1993 \\
\text { June }\end{array}$ & $\begin{array}{l}1993 \\
\text { July } \\
\end{array}$ & $\begin{array}{l}1993 \\
\text { Aug. }\end{array}$ & $\begin{array}{l}1993 \\
\text { Sep. }\end{array}$ & $\begin{array}{l}1993 \\
\text { Oct. }\end{array}$ & $\begin{array}{l}1993 \\
\text { Nov. }\end{array}$ & $\begin{array}{l}1993 \\
\text { Dec. }\end{array}$ & $\begin{array}{c}4093 \\
\text { TOTAL HCf }\end{array}$ & $\begin{array}{c}\text { YTo } \\
\text { Total Mcf }\end{array}$ \\
\hline $\begin{array}{l}\text { YANKEE GAS SERVICES CO. } \\
\text { (ALBERTA NORTHEAST GAS (WGM LTD. 2)) } \\
\text { AVG Daily Quantity } \\
\text { Total Price (Waddington, New York) } \\
\text { Commodity Component } \\
\text { Demand Component } \\
\text { Reservation Fee Component }\end{array}$ & 11.3 & $\begin{array}{l}11.3 \\
2.91 \\
1.98 \\
0.93 \\
0.00\end{array}$ & $\begin{array}{r}11.3 \\
-2.91 \\
1.96 \\
0.95 \\
0.00\end{array}$ & $\begin{array}{l}11.3 \\
2.91 \\
1.95 \\
0.96 \\
0.00\end{array}$ & $\begin{array}{l}11.3 \\
2.47 \\
1.49 \\
0.98 \\
0.00\end{array}$ & $\begin{array}{l}11.3 \\
2.70 \\
1.73 \\
0.97 \\
0.00\end{array}$ & $\begin{array}{l}11.3 \\
2.37 \\
1.39 \\
0.98 \\
0.00\end{array}$ & $\begin{array}{l}11.3 \\
2.37 \\
1.39 \\
0.98 \\
0.00\end{array}$ & $\begin{array}{l}11.3 \\
2.37 \\
1.40 \\
0.97 \\
0.00\end{array}$ & $\begin{array}{l}11.3 \\
2.37 \\
1.43 \\
0.94 \\
0.00\end{array}$ & $\begin{array}{l}11.3 \\
2.29 \\
1.35 \\
0.94 \\
0.00\end{array}$ & $\begin{array}{l}11.3 \\
2.66 \\
1.73 \\
0.93 \\
0.00\end{array}$ & $\begin{array}{l}11.3 \\
2.69 \\
1.77 \\
0.92 \\
0.00\end{array}$ & $1,035,031$ & $4,117,531$ \\
\hline $\begin{array}{l}\text { YANKEE GAS SERVICES CO. } \\
\text { (ALBERTA NORTHEAST GAS (PROGAS)) } \\
\text { AVG Daily Quant ity } \\
\text { Total Price (Waddington, New York) } \\
\text { Commodity Component } \\
\text { Demand Component } \\
\text { Reservation Fee Component }\end{array}$ & 9.9 & $\begin{array}{l}9.9 \\
2.91 \\
1.97 \\
0.94 \\
0.00\end{array}$ & $\begin{array}{l}9.9 \\
2.91 \\
1.95 \\
0.96 \\
0.00\end{array}$ & $\begin{array}{l}9.9 \\
2.91 \\
1.97 \\
0.94 \\
0.00\end{array}$ & $\begin{array}{r}9.9 \\
2.46 \\
1.48 \\
0.98 \\
0.00\end{array}$ & $\begin{array}{r}9.9 \\
2.76 \\
1.78 \\
0.98 \\
0.00\end{array}$ & $\begin{array}{l}9.9 \\
2.37 \\
1.39 \\
0.98 \\
0.00\end{array}$ & $\begin{array}{l}9.9 \\
2.37 \\
1.38 \\
0.99 \\
0.00\end{array}$ & $\begin{array}{r}9.9 \\
2.37 \\
1.40 \\
0.97 \\
0.00\end{array}$ & $\begin{array}{r}9.9 \\
2.36 \\
1.42 \\
0.94 \\
0.00\end{array}$ & $\begin{array}{r}9.9 \\
2.30 \\
1.35 \\
0.95 \\
0.00\end{array}$ & $\begin{array}{l}9.9 \\
2.67 \\
1.73 \\
0.94 \\
0.00\end{array}$ & $\begin{array}{l}9.9 \\
2.69 \\
1.73 \\
0.96 \\
0.00\end{array}$ & 910,800 & $3,623,400$ \\
\hline $\begin{array}{l}\text { YANKEE GAS SERVICES CO. } \\
\text { (ALBERTA NORTHEAST GAS (ATCOR)) } \\
\text { AVG Daily Quant ity } \\
\text { Total Price (Waddington, New York) } \\
\text { Commodity Component } \\
\text { Demand Component } \\
\text { Reservation Fee Component }\end{array}$ & 5.2 & $\begin{array}{r}5.2 \\
2.91 \\
2.01 \\
0.90 \\
0.00\end{array}$ & $\begin{array}{l}5.2 \\
2.91 \\
2.01 \\
0.90 \\
0.00\end{array}$ & $\begin{array}{l}5.2 \\
2.92 \\
2.01 \\
0.91 \\
0.00\end{array}$ & $\begin{array}{l}5.2 \\
2.46 \\
1.54 \\
0.92 \\
0.00\end{array}$ & $\begin{array}{l}5.2 \\
2.71 \\
1.78 \\
0.93 \\
0.00\end{array}$ & $\begin{array}{l}5.2 \\
2.37 \\
1.45 \\
0.92 \\
0.00\end{array}$ & $\begin{array}{l}5.2 \\
2.37 \\
1.46 \\
0.91 \\
0.00\end{array}$ & $\begin{array}{l}5.2 \\
2.37 \\
1.46 \\
0.91 \\
0.00\end{array}$ & $\begin{array}{r}5.2 \\
2.37 \\
1.48 \\
0.89 \\
0.00\end{array}$ & $\begin{array}{l}5.2 \\
2.30 \\
1.42 \\
0.88 \\
0.00\end{array}$ & $\begin{array}{l}5.2 \\
2.66 \\
1.79 \\
0.87 \\
0.00\end{array}$ & $\begin{array}{l}5.2 \\
2.68 \\
1.80 \\
0.88 \\
0.00\end{array}$ & 480,424 & $1,910,834$ \\
\hline $\begin{array}{l}\text { YANKEE GAS SERVICES CO. } \\
\text { (ALBERTA NE GAS (AEC OIL \& GAS COMPANY)) } \\
\text { AVG Daily Quantity } \\
\text { Total Price (Waddington, New York) } \\
\text { Commodity Component } \\
\text { Demand Component } \\
\text { Reservation Fee Component }\end{array}$ & 3.9 & $\begin{array}{r}3.9 \\
2.91 \\
2.02 \\
0.89 \\
0.00\end{array}$ & $\begin{array}{l}3.9 \\
2.92 \\
2.01 \\
0.91 \\
0.00\end{array}$ & $\begin{array}{l}3.9 \\
2.91 \\
2.01 \\
0.90 \\
0.00\end{array}$ & $\begin{array}{l}3.9 \\
2.46 \\
1.53 \\
0.93 \\
0.00\end{array}$ & $\begin{array}{l}3.9 \\
2.71 \\
1.75 \\
0.96 \\
0.00\end{array}$ & $\begin{array}{l}3.9 \\
2.37 \\
1.43 \\
0.94 \\
0.00\end{array}$ & $\begin{array}{r}3.9 \\
2.37 \\
1.45 \\
0.92 \\
0.00\end{array}$ & $\begin{array}{l}3.9 \\
2.37 \\
1.42 \\
0.95 \\
0.00\end{array}$ & $\begin{array}{l}3.9 \\
2.37 \\
1.48 \\
0.89 \\
0.00\end{array}$ & $\begin{array}{l}3.9 \\
2.29 \\
1.47 \\
0.82 \\
0.00\end{array}$ & $\begin{array}{l}3.9 \\
2.66 \\
1.78 \\
0.88 \\
0.00\end{array}$ & $\begin{array}{r}3.9 \\
2.68 \\
1.79 \\
0.89 \\
0.00\end{array}$ & 361,376 & $1,437,618$ \\
\hline
\end{tabular}




\section{VOLURE AID PRICE REPORT}

Iong Ter Exports

Volumes (MMCf/d) \& Prices ( $\$$ /MMBTU) of Natural

Gas Exported During the Past 12 Months $01 / 01 / 93-12 / 31 / 93$

Long-Term Exporter

CENTRA GAS MANITOBA (1)

(TRANSCANADA PIPELINES LIMITED)

AVG Daily Quantity

Total Price (Noyes, Minnesota)

Commodity

Reservation fee component

MARATHON OIL COMPANY *

(TOKYO ELECTRIC CO./TOKYO GAS CO.)

AVG Daily Quantity

Total Price (Kenai, Alaska)

Commodity Component

Reservation fee Component

PHILLIPS ALASKA NATURAL GAS CORPORATION * (TOKYO ELECTRIC CO./TOKYO GAS CO.)

AVG Daily Quantity

Total Price (Kenai, Alaska)

Commodity Component

Demand Component

Reservation Fee Component

Total Mcf

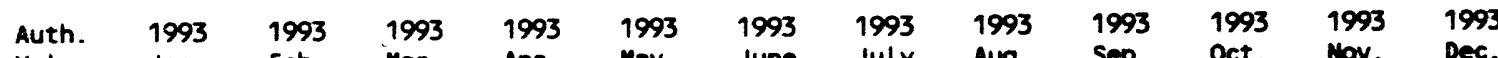

Vols. Jan. Feb. Mar. Apr. May June July Aug. Sep. Oct. Nov. Dec.

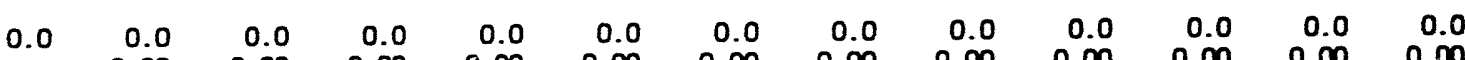

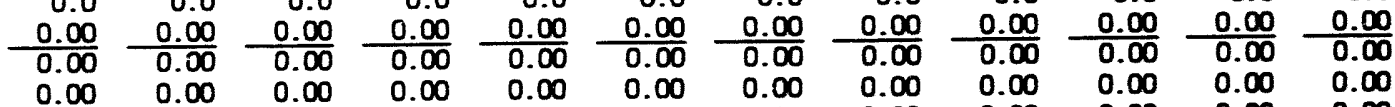

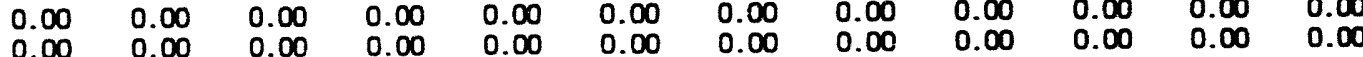

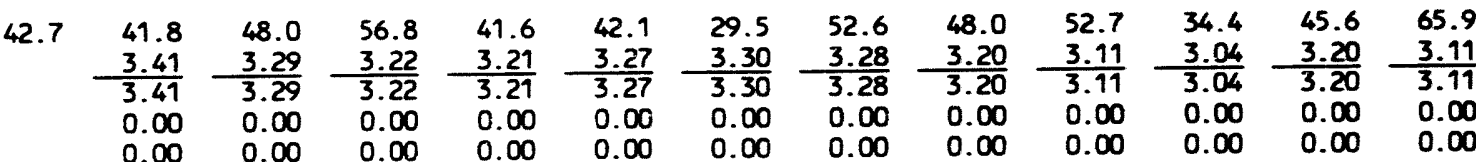

115.3

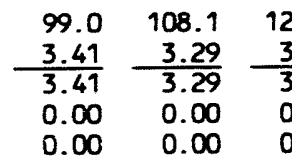

$\begin{array}{llllllllll}9.9 & 104.2 & 99.2 & 68.8 & 113.9 & 105.6 & 118.5 & 72.2 & 113.0 & 146.7\end{array}$

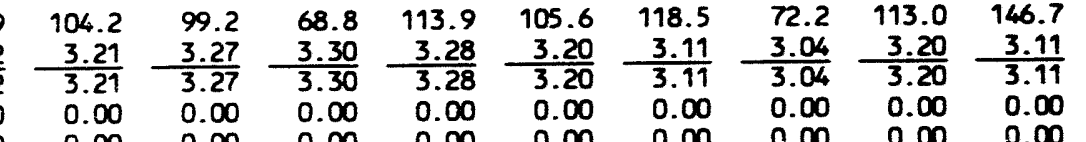

$10,175,100 \quad 38,927,040$

$4,479,040 \quad 17,019,117$ 4093
TOTAL HCf TOTAL HCf

* Price for LNG at the delivery point in Japan.

(1) Pursuant to a gas purchase agreement with Coastal Gas Marketing, Centra is exporting, via backhaul, up to 13.5 Bcf thru $3 / 31 / 95$. 
SHORT-TERM BLANKET IMPORTS

$\begin{array}{lccc}\begin{array}{l}\text { Year \& } \\ \text { Month }\end{array} & \text { Active } & \text { Est. Volumes } & \text { Weighted Average } \\ \text { Importers } & \underline{\text { Price (\$MMMTU) }}\end{array}$

$\begin{array}{llll}\text { 1991 } & & & \\ \text { January } & 55 & 50151 & 1.80 \\ \text { February } & 53 & 47220 & 1.58 \\ \text { March } & 47 & 52918 & 1.47 \\ \text { April } & 60 & 52947 & 1.33 \\ \text { May } & 60 & 51716 & 1.33 \\ \text { June } & 57 & 57147 & 1.32 \\ \text { July } & 54 & 50762 & 1.28 \\ \text { August } & 49 & 49395 & 1.23 \\ \text { September } & 55 & 52993 & 1.34 \\ \text { October } & 56 & 55510 & 1.46 \\ \text { November } & 56 & 61718 & 1.54 \\ \text { December } & 60 & 64029 & 1.65\end{array}$

$1992 *$

January

63000

February

75904

1.22

March

75777

1.21

April

61

74181

1.29

May

69389

1.38

63415

$65443 \quad 1.35$

July

65

59709

1.52

August

54319

1.58

September

63015

1.89

November

70750

1.74

December

65

64330

1.83

1993 *

January 64

59879

1.84

February

62911

1.58

March

70845

1.67

April

61000

1.71

May

53853

1.84

June

53821

1.62

July

63432

1.58

August

70638

1.62

September
October

62626

1.80

61610

1.67

November

88408

1.93

December

101012

2.15

- Figures have been revised 
Page - 48

SHORT-TERM IMPORTERS

Estimated Volumes (MMCF)

Oct.-Dec.

ACCESS ENERGY CORPORATION

AIG TRADING CORPORATION

ALUMINUM COMPANY OF AMERICA

AMERICAN HUNTER EXPLORATION LTO.

AMOCO CANADA MARKETING CORPORATION

AMOCO ENERGY TRADING CORPORATION

ANR GAS SUPPLY COMPANY

ANR PIPELINE COMPANY

AQUILA ENERGY MARKETING CORPORATION

ARCO PRODUCTS COMPANY

BAY STATE GAS COMPANY

BOSTON GAS COMPANY

BOW VALLEY ENERGY MARKETING INC.

BRIDGEGAS U.S.A. INC.

BROAD STREET OIL \& GAS COMPANY

BRYMORE ENERGY INC.

CANADA IMPERIAL OIL LIMITED

CANADIAN HYOROCARBONS MARKETING (U.S.) INC.

CANADIANOXY MARKETING INC.

CANSTATES PETROLEUM MARKETING

CANWEST GAS SUPPLY U.S.A., INC.

CASCADE NATURAL GAS CORPORATION

CHEVRON NATURAL GAS SERVICES INC.

CIBOLA CORPORATION

CMEX ENERGY, INC.

CNG PRODUCING COMPANY

COASTAL GAS MARKETING COMPANY

COENERGY TRADING COMPANY

CONOCO, INC.

CONTINENTAL ENERGY MARKETING LTD.

CRESTAR ENERGY MARKETING CORPORATION

CU ENERGY MARKETING, INC.

DARTMOUTH POWER ASSOCIATES L.P.

DEKALB ENERGY COMPANY

DEVELOPMENT ASSOCIATES, INC.

DIRECT ENERGY MARKETING LIMITED

ENRON GAS MARKETING, INC.

GOETZ OIL CORPORATION

GRAND VALLEY GAS COMPANY

GRANITE STATE GAS TRANSMISSION, INC.

GREAT FALLS GAS COMPANY

GREAT PLAINS MATURAL GAS COMPANY

GREAT WEST ENERGY LTD.

HOWARD ENERGY COMPANY, INC.

HUSKY GAS MARKETING, INC.

IGI RESOURCES INC.
1992

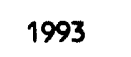

Jan. -Mar.

692

0

139

4197

509

116

12392

149

1014

0
1082

1082

0

0

699

0
4208

258

15640

3746

0

0

0
4562

965

0

4627

0

4
0

28

238

107

3293

4

12006

555

17
0

0
106

0

7071

3673

69

0

0
1249

0

0

17345

0

1330

0

0

0

0

186

53

0

3433

0

17

16702

4097

0

78

0

0
7749

314

0

5977

0

1320

336

0

340

10

4216

29

6747

543

75

0
165

165
0

9683

4920
1993

Apr.-June

\section{0}

0
0

1205

0

0

19483

0

768

0

0

127

489

0

0
4068

0

32

10538

4121

0

19

0

0
858

583

0

2837

0

605

107

0

0

58
4885

4885

10028

549

97

0

166

75

7997

4446
1993

July-Sep.
1993

Oct.-Dec.

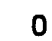

795

2438

3582

0

315

8707

0

774

376

0

0

15

387

0

608

2697

0

0

12739

3549

4407

148

725

235

1688

484

389

1944

842

155

175

716

609

337

6122

17

13824

30

439

35

639

210

9208

5016 
SHORT-TERM IMPORTERS

Estimated Volumes (MMCF)
- cont. --

$\begin{array}{ccccc}1992 & 1993 & 1993 & 1993 & 1993 \\ \text { Oct.-Dec. } & \text { Jan.-Mar. } & \text { Apr.-June } & \text { July-Sep. } & \text { Oct.-Dec. }\end{array}$

INDECK ENERGY SERVICES, INC.

INLAND NATURAL GAS MARKETING LTD.

INTALCO ALUMINUM CORPORATION

INTERENERGY CORPORATION

INTERSTATE POWER COMPANY

IOWA ELECTRIC LIGHT \& POWER COMPANY

IROQUOIS ENERGY MANAGEMENT, INC.

JMC FUEL SERVICES, INC.

JONAN GAS MARKETING

KCS ENERGY MARKETING, INC.

KIMBALL ENERGY CORPORATION

LOUIS DREYFUS ENERGY CORPORATION

MADISON GAS \& ELECTRIC COMP' IY

MASSPOWER

MCV GAS ACQUISITION GENERAL PARTNERSHIP

METROPOLITAN UTILITIES DISTRICT

MIDWEST GAS COMPANY

MINNEGASCO

MOBIL NATURAL GAS INC.

MOCK RESOURCES, INC.

MURPHY GAS GATHERING

MURPHY OIL COMPANY LIMITED

NATIONAL STEEL CORPORATION

NATURAL GAS CLEARINGHOUSE INC.

NIAGARA MOHAWK POWER CORPORATION

NORCEN MARKETING INC.

NORTH AMERICAN RESOURCES COMPANY

NORTH CANADIAN MARKETING CORPORATION

NORTHERN ILLINOIS GAS COMPANY

NORTHERN MINNESOTA UTILITIES

NORTHERN STATES POWER COMPANY (MINNESOTA)

NORTHERN STATES POWER COMPANY (WISCONSIN)

536

1694

0

1595

NORTHERN UTILITIES, INC.

MORTHRIDGE GAS MARKETING, INC.

NORTHWESTERN PUBLIC SERVICE COMPANY

OREGON NATURAL GAS DEVELOPMENT CORPORATION

ORYX GAS MARKETING, L.P.

PACIFIC GAS \& ELECTRIC COMPANY

PACIFIC GAS TRANSMISSION COMPANY

PAN NATIONAL GAS SALES, INC. (1)

PAN-ALBERTA GAS (U.S.), INC.

PANHANDLE TRADING COMPANY

PARAMOUNT RESOURCES U.S., INC.

PAWTUCKET POWER ASSOCIATES L.P.

PEOPLES NATURAL GAS COMPANY

PEPPERELL PONER ASSOCIATES L.P.
536

534

143

46

0

0

0

0

0
445

3656

0

0

0

0
4348

0

0

1264

3655

0

453
6585

0

330

0

534

0
138

14

0

0

0

0

0

129

4949

1891

0

0

363

0

0

0
4759

8

0

0

1706

792

0

4

138

2773

0

1042

0

0

$\begin{array}{rr}0 & 0 \\ 1815 & 2184\end{array}$

$0 \quad 0$

1228

0

0

0

$209 \quad 2137$

$8681 \quad 4988$

$0 \quad 371$

000

$\begin{array}{rr}0 & 72 \\ 607 & 500\end{array}$

$39 \quad 122$

$$
315
$$

126

117

0

0

0

50

0

177

3263

829

0

12

0

0

0
0

3672

0

0

1576

63

0

9

3981

0
261

0

0
0

2143

0

135

0

0

111

4157

0

0

337

96

145

\section{7}

0

127

243

0

$0 \quad 168$

$0 \quad 384$

$40 \quad 0$

$0 \quad 49$

$653 \quad 294$

$3528 \quad 1976$

606

0

0

0

0

4356

4356

495

0

0
1500

1164

0

824

5410

0

334

0

0

0
2488

0

171

0

1297

0

4163

4163

0

0

65

141

60

0

0

416

397

1627

7220

234

101

97

1714

538

0

297

7192

362

1148

1201

204

58

2875

58

55

86

0

146

281

7794

160

1196

23

800
366

364

212

78

40 4 9

43

(1) These figures represent sales for resale rather than imports. Actual imports are as follows: 4th atr 92 (332); 1st atr 93 (5134); 2nd atr 93 (103); 3rd atr 93 (179); 4th atr 93 (452). 
Page - 50

SHORT-TERM IMPORTERS

Estimated Volumes (MMCF)

1992

Oct.-Dec.

1993

PETRO-CANADA HYDROCARBONS, INC. PGRE GAS SUPPLY BUSINESS UNIT POCO PETROLEUM, INC.

PORTLAND GENERAL ELECTRIC COMPANY PROGAS U.S.A., INC.

REDWOOD RESOURCES INC.

RENAISSANCE ENERGY (U.S.), INC. SALMON RESOURCES LTD.

SAN DIEGO GAS \& ELECTRIC SANTANNA NATURAL GAS CORPORATION SELKIRK COGEN PARTNERS, L.P. SIERRA PACIFIC POWER COMPANY SOUTHEASTERN MICHIGAN GAS COMPANY SOUTHERN CALIFORNIA EDISON COMPANY SOUTHWEST GAS CORPORATION SUNCOR INC.

TARPON GAS MARKETING LTD. TENNECO GAS MARKETING COMPANY TENNESSEE GAS PIPELINE COMPANY TEXAS-OHIO GAS, INC.

TEXPAR ENERGY, INC.

THE MONTANA POWER COMPANY TRANSCO ENERGY MARKETING COHPANY TRISTAR GA : MARKETING COMPANY UNIVERSAL RESOURCES CORPORATION VALERO INDUSTRIAL GAS, L.P. VECTOR ENERGY (U.S.A.) INC. VERMONT GAS SYSTEHS, INC. VICTORIA GAS CORPORATION WASHINGTON ENERGY MARKETING, INC. WASHINGTON NATURAL GAS COMPANY WES CANA ENERGY MARKETING (U.S.) INC. WESTAR MARKETING COMPANY WESTCOAST GAS SERVICES INC. WESTERN GAS MARKETING INC. WESTERN GAS RESOURCES, INC. WILLIAMS GAS MARKETING COMPANY WINDWARD ENERGY \& MARKETING COMPANY WISCONSIV GAS COMRANY WISCONSIN POWF': \& LIGHT COMPANY WISCONSIN MLIC SERVICE CORPORATION

\begin{tabular}{|c|c|c|c|c|}
\hline 2964 & 3252 & 2456 & 2479 & 3820 \\
\hline 0 & 0 & 0 & 0 & 35124 \\
\hline 6768 & 5867 & $=179$ & 6512 & 6495 \\
\hline 781 & 0 & 0 & 0 & 21 \\
\hline 5970 & 4934 & 10073 & 9887 & 6302 \\
\hline 109 & 399 & 0 & 0 & 0 \\
\hline 0 & 300 & 902 & 1526 & 2553 \\
\hline 416 & 338 & 145 & 315 & 3420 \\
\hline 0 & 0 & 0 & 0 & 214 \\
\hline 2250 & 2650 & 2291 & 1669 & 355 \\
\hline 0 & 18 & 0 & 0 & 0 \\
\hline 2699 & 2662 & 2700 & 2704 & 2718 \\
\hline 111 & 78 & 41 & 0 & 84 \\
\hline 0 & 0 & 0 & 0 & 3175 \\
\hline 0 & 0 & 0 & 62 & 146 \\
\hline 6091 & 6443 & 6473 & 6365 & 7549 \\
\hline 0 & 0 & 0 & 0 & 87 \\
\hline 617 & 586 & 720 & 305 & 0 \\
\hline 6540 & 4866 & 5926 & 5633 & 1653 \\
\hline 109 & 242 & 280 & 0 & 0 \\
\hline 0 & 44 & 38 & 0 & 0 \\
\hline 262 & 494 & 0 & 0 & 153 \\
\hline 805 & 2090 & 1315 & 3132 & $401:$ \\
\hline 0 & 0 & 0 & 271 & 568 \\
\hline 668 & 983 & 525 & 1027 & 179 \\
\hline 0 & 0 & 0 & 0 & 133 \\
\hline 2881 & 2757 & 2634 & 2181 & 1643 \\
\hline 17 & 216 & 0 & 0 & 52 \\
\hline 318 & 409 & 1153 & 0 & 0 \\
\hline 0 & 0 & 449 & 440 & 4153 \\
\hline 106 & 135 & 760 & 1161 & 597 \\
\hline 9038 & 7592 & 8481 & 11024 & 7814 \\
\hline 332 & 0 & 0 & 0 & 0 \\
\hline 4076 & 764 & 1920 & 4329 & 2713 \\
\hline 22555 & 26793 & 17263 & 14146 & 18406 \\
\hline 285 & 389 & 374 & 1156 & 262 \\
\hline 1114 & 264 & 0 & 0 & 0 \\
\hline 10 & 0 & 146 & 261 & 25 \\
\hline 0 & 0 & 0 & 0 & 7683 \\
\hline 290 & 222 & 0 & 346 & 0 \\
\hline 486 & 718 & 710 & 730 & 732 \\
\hline 198096 & 193634 & 168674 & 196697 & 251030 \\
\hline
\end{tabular}


Page - 51

PURCHASERS OF SHORT-TERM GAS

Estimated Volumes Imported (MMCF)

Oct. -Dec.

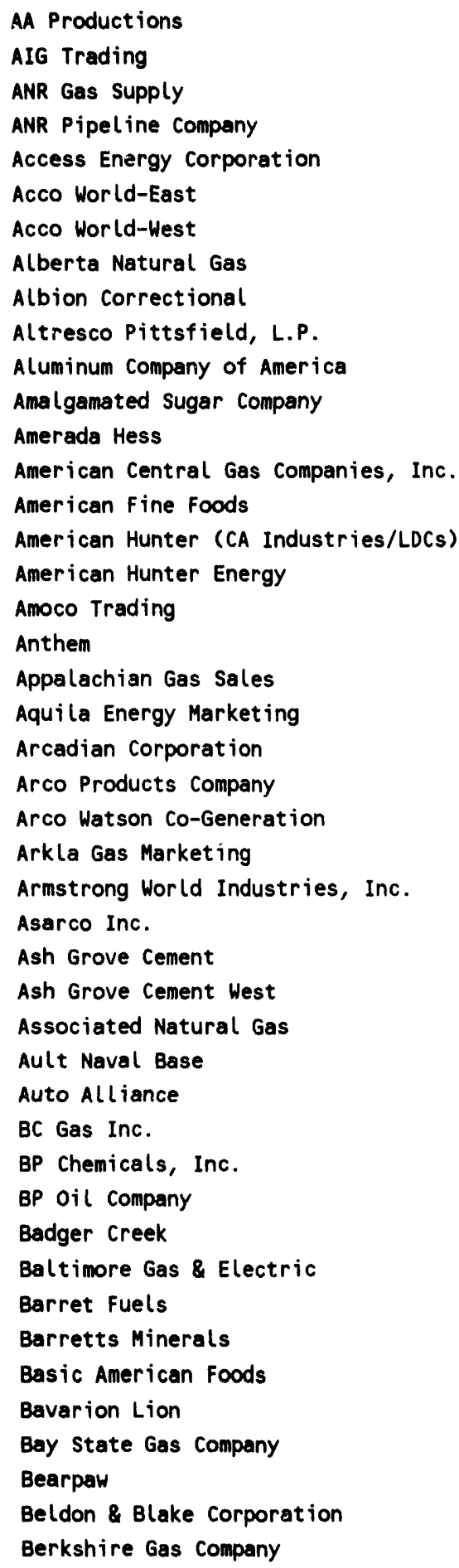

1992

$1993 \quad 1993$

Jan. - Mar<smiles>CCC</smiles><smiles>CCC</smiles>

12392

759

2

2

0

22

3223

231

0

61

65

2

0

874

0

0

75

2820

280

1443

0

0

106

78

69

0

0

47

0

0

776

214

23

188

147

0

73

0

33

0

41

131

0

61

0

0

4
1993

July-Sep.
1993

Oct.-Dec.

$\begin{array}{rr}50 & \\ 109 & 19 \\ 0 & \\ 19483 & 188 \\ 922 & 364 \\ 2 & \\ 3 & \\ 0 & \\ 0 & \\ 2855 & \\ 134 & 2471 \\ 38 & \\ 0 & \\ 0 & \\ 23 & \\ 143 & \\ 145\end{array}$

67

192

0

18861

364

2

3

7

13

2471

89

1

0$$
24
$$

1398

1398
0

420

97

4342

397

1083

835

202

188

93

47

122

0

36

156

0

0

132

51

89

12

0

64
0

85

315

8707

0

2

3

0

0

739

189

11

0

0

8

0
970

3907

0

330

2063

0

1247

368

109

110

75

101

0

40

0

396

0

45

0

431

0

86

43

0

376

12

0 


\section{PURCHASERS OF SHORT-TERM GAS \\ Estimated Volumes Imported (MMCF)}

$\begin{array}{ccccc}1992 & 1993 & 1993 & 1993 & 1993 \\ \text { Oct.-Dec. } & \text { Jan.-Mar. } & \text { Apr.-June } & \text { July-Sep. } & \text { Oct.-Dec. }\end{array}$

Bethlehem Steel Corporation

Biomass

Blaw Knox

Blue Lake Storage

Boeing Company

Boise Cascade Corporation

Boston Edison Company

Boston Gas

Boundary Gas Inc.

Bridge Oil

Bridgegas

Broad Street oil \& Gas

Brooklyn Interstate Natural Gas

Brymore Energy Inc.

C Energy, Inc.

C.P. National Corporation

CMS Gas Marketing

CNF Transmission

CNG Gas Services

CNG Producing Company

CNG Transmission Corporation

Calaveras

Canada Imperial Oil Limited

Canadian Hydrocarbons

Canadianoxy Marketing Inc.

Canstates Gas Marketing

Canton Central School

Canton-Potsdam Hospital

Canwest Gas Supply

Carborundum Abrasives

Carnation Dairies

Cascade Natural Gas

Cascade Steel

Caterpillar Inc.

Central Hudson Gas \& Electric

Central Islip

Centran

Chautauqua Energy Marketing Inc.

Chevron Natural Gas Services, Inc.

Chevron U.S.A. Inc.

114

0

1

256

333

0

1082

0

201

514

2680

591

0

0

0

1552

24

3

0

28

4566

176

3483

2303

Cibola/Peoples Natural Gas/No. Illinois Gas

Cincinatti Gas \& Electric

Citizens Gas Supply Corporation

City Ogdensburg, NY

city of Buckley, OR
51

0

0

934

768

18

34

3

207

0

935

0

11

42

0

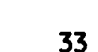

0

0

0

237

1113

0

0

123

89

404

739

3684

0

0

0

0

0

0

0

22

0

0

23

118

5

5

0

0

38

4407$$
\begin{array}{r}
0 \\
41
\end{array}
$$

41
344

344
0

0

18

0

167

5102

0

62

805

0

0$$
45
$$$$
0
$$

Apr.-June

0

$$
\begin{array}{r}
0 \\
294 \\
2
\end{array}
$$$$
\begin{aligned}
& 2 \\
& 0
\end{aligned}
$$$$
335
$$$$
753
$$$$
5
$$$$
0
$$$$
29
$$$$
447
$$$$
1038
$$$$
293
$$$$
3342
$$$$
46
$$$$
0
$$$$
0
$$$$
0
$$$$
\begin{array}{r}
0 \\
358
\end{array}
$$$$
0
$$$$
1
$$$$
0
$$$$
0
$$$$
\begin{array}{r}
246 \\
0
\end{array}
$$$$
234
$$$$
5
$$$$
5
$$$$
0
$$$$
0
$$$$
182
$$$$
4869
$$$$
162
$$$$
0
$$$$
26
$$$$
0
$$$$
0
$$$$
20
$$$$
0
$$$$
413
$$$$
2111
$$$$
0
$$$$
0
$$$$
326
$$

Oct.-DeC.

$\begin{array}{rr}1 & 31 \\ 152 & 109 \\ 0 & 1 \\ 0 & 116 \\ 339 & 732 \\ 824 & 526 \\ 0 & 0 \\ 0 & 0 \\ 0 & 0 \\ 167 & 0 \\ 2874 & 1276 \\ 1102 & 542 \\ 2734 & 1014 \\ 0 & 47 \\ 0 & 305 \\ 0 & 0 \\ 0 & 0 \\ 0 & 108 \\ 835 & 1786 \\ 0 & 235 \\ 0 & 356 \\ 0 & 0 \\ 0 & 608 \\ 282 & 52 \\ 0 & 0 \\ 8 & 34 \\ 5 & 5 \\ 5 & 5 \\ 116 & 0 \\ 13 & 14 \\ 54 & 165 \\ 5006 & 6357 \\ 155 & 141 \\ 0 & 0 \\ 11 & 10 \\ 15 & 0 \\ 0 & 0 \\ 69 & 0 \\ 0 & 4407 \\ 72 & 340 \\ 5367 & 5571 \\ 0 & 0 \\ 0 & 0 \\ 0 & 0 \\ 0 & \\ 0 & \end{array}$


Page - 53

PURCHASERS OF SHORT-TERM GAS

Estinated Volumes Imported (MMCF)

$\begin{array}{ccccc}1992 & 1993 & 1993 & 1993 & 1993 \\ \text { Oct.-Dec. } & \text { Jan.-Mar. } & \text { Apr.-June } & \text { July-Sep. } & \text { Oct.-Dec. }\end{array}$

City of Ellensburg, WA

City of Enumclaw, WA

City of Fayetteville, NC

118

City of Greenwood, SC

City of Long Beach, CA

City of Morehead, KY

City of Ogdensburg School District

City of Ogdensburg Water Pollution Control

City of Palo Alto, CA

City of Perham, MN

Clarkson University

118

145

Clay Basin Storage

Clinton Gas

CoWest Energy

Coastal Gas Marketing Company

Coenergy Trading Company

coenergy Ventures

college of Technology

Colonial Gas Company

Columbia Falls

Commonweal th Gas Company

Connecticut Natural Gas

Conoco Inc.

Consolidated Edison

Consolidated Fuel

Consumers Power Company

Consumers' Gas Company Ltd.

Continental Energy Marketing Ltd.

cook Inlet

Cornell Linen

Crysen

DGS Trading

Darigold Inc.

Darling Delaware

Delmarva Power \& Light Company

Destec

Destec Energy DBL "C"

Development Associates

Dexel

Direct Energy

Direct Gas

Domtar Gypsum

Doswell L.P.

Dresser Rand

ESCO Corporation

Eagle Picher

\begin{tabular}{|c|c|}
\hline 0 & 0 \\
\hline 0 & 0 \\
\hline 0 & 0 \\
\hline 0 & 0 \\
\hline 1 & 0 \\
\hline 6 & 8 \\
\hline 1 & 2 \\
\hline 0 & 0 \\
\hline 123 & 176 \\
\hline 0 & 39 \\
\hline 0 & 0 \\
\hline 0 & 0 \\
\hline 0 & 0 \\
\hline 9489 & 4708 \\
\hline 965 & 1309 \\
\hline 0 & 0 \\
\hline 0 & 21 \\
\hline 8 & 156 \\
\hline 44 & 48 \\
\hline 13 & 5 \\
\hline 667 & 672 \\
\hline 0 & 39 \\
\hline 0 & 954 \\
\hline 20 & 0 \\
\hline 0 & 0 \\
\hline 0 & 0 \\
\hline 0 & 0 \\
\hline 0 & 0 \\
\hline 0 & 6 \\
\hline 25 & 0 \\
\hline 39 & 57 \\
\hline 0 & 0 \\
\hline 2 & 3 \\
\hline 0 & 0 \\
\hline 0 & 0 \\
\hline 46 & 0 \\
\hline 2682 & 2800 \\
\hline 0 & 0 \\
\hline 2310 & 921 \\
\hline 18 & 183 \\
\hline 0 & 0 \\
\hline 0 & 0 \\
\hline 2 & 0 \\
\hline 40 & 39 \\
\hline 50 & 52 \\
\hline
\end{tabular}

98
0
0
0
0
1
9
2
0
124
39
489
0
0

118

$0 \quad 50$

$0 \quad 23$

$0 \quad 22$

164

0

9

3

125

40

227

189

5522

0
4423

831

3470

0

0

0

0

770

152

40

112

339

500

4897

582

1027

21

12

29

0

144

0

13

0
6898

0

648

0
21

21

0

0

60

0

352

206

389

$\begin{array}{rr}2 & 94 \\ 0 & 0\end{array}$

$6900 \quad 0$

164

806

523

1196

6

0

663

20

0

0

$\begin{array}{rr}212 & 122 \\ 0 & 0\end{array}$

$3089 \quad 1804$

$174 \quad 286$

$123 \quad 925$

$0 \quad 56$

$183 \quad 184$

2950

$0 \quad 0$

$35 \quad 20$

6

0 
Page - 54

\section{PURCHASERS OF SHORT-TERM GAS \\ Estimated Volumes Imported (MMCF)}

$\begin{array}{ccccc}1992 & 1993 & 1993 & 1993 & 1993 \\ \text { Oct.-Dec. } & \text { Jan.-Mar. } & \text { Apr.-June } & \text { July-Sep. } & \text { Oct.-Dec. }\end{array}$

Eastex Energy

Eastex Hydrocarbons

El Paso Natural Gas

Elf Atochem

Elizabethtown Gas Company

Empire Natural Gas Corporation

Encogen Northwest, L.P.

Endurance Fruit

Energy Marketing Exchange

Energynorth Inc.

Engroup Inc.

Enmark Gas Corporation

Enron Gas Marketing

Ensearch Development Corporation

Entrade Corporation

Equitable Resources

Escanaba Paper

Essex County Gas Company

Evanite

FMC

Falcon Seaboard

Fall River Gas

Flanders Inn

Ford Motor Company

Fort Lewis

Gas Energy Development

Gaslantic

Gasmark Inc.

Gaylord Container Corporation

Gaz Metropolitan

General Chemical

Georgia Pacific Corporation

Gilroy Energy Company

Global Petroleum

Goetz Energy Corporation

Grand Valley Gas Company

Granite State Gas Transmission, Inc.

Great Falls Gas Company

Great Lakes Gas Transmission Co.

Great Plains Natural Gas Company

Greeley Gas Company

Gulf Gas

Hadson Gas Systems

Hazelton Fuel Management Company

Helen Hayes Center

Helm. \& Payne
Oct.-DeC.

Jan. - Ma

98

98

62

0

0

61

0

0

0

0

35

0
160

160
1948

0

650

0

149

30

0

0
273

8

\section{0}

0

1

0

53

0

0

0

0

0

20

10

2255

0

0

2

0

$$
0
$$$$
0
$$$$
0
$$$$
0
$$$$
1
$$$$
0
$$$$
0
$$$$
0
$$$$
78
$$$$
0
$$$$
0
$$$$
0
$$$$
0
$$$$
0
$$$$
0
$$$$
0
$$$$
\begin{array}{r}
0 \\
29
\end{array}
$$$$
4215
$$$$
543
$$$$
75
$$$$
613
$$$$
910
$$$$
40
$$$$
0
$$$$
536
$$$$
0
$$$$
\begin{array}{r}
0 \\
743
\end{array}
$$

July-Sep.

oct.-Dec. 
Page - 55

PURCHASERS OF SHORT-TERM
Estimated
$\begin{array}{ccccc}1992 & 1993 & 1993 & 1993 & 1993 \\ \text { Oct.-Dec. } & \text { Jan.-Mar. } & \text { Apr.-June } & \text { July-Sep. } & \text { Oct.-Dec. }\end{array}$

Hesse Gas Company

Heuvelton Central School

High Sierra

Highland Nursing Home

Holman

Hoosier Magnetics, Inc.

Hopewell Cogeneration Facility

Howard Energy Company

Hub Services

IBP

ICC

ICG Utilities

IESCO

IGI Resources

Illinois Institute of Technology

Illinois Power

Indeck Energy Services

Indeck Energy Services of Oswego, Inc.

Indeck Energy Services of Yerkes, Inc.

Industrial Energy Applications

Inland Steel Company

Intal co Aluminum Corporation

Interenergy Corporation

intermountain Pipeline

International Paper

Interox

Interstate Power Company

Inverness Petroleum

Lowa Electric Light \& Power

J. Aaron Company

J. Makowski

J.H. Gypsum

J.R. Simplot company

JMC Fuel Services

James River Corporation

Jefferson Smurfit

Joanna A. CHF Company

John Brown Oil \& Gas

Jonan Gas Marketing

K-N Gas Marketing

KCS Energy Management

KOGAS

KTM

Kamine/Besicorp Carthage L.P.

Kamine/Besicorp Natural Dam L.P.

Kamine/Besicorp South Glens Falls

83
0
11
11
0
16
76
399
0
81
0
160
310
9989

69

207

$\begin{array}{rr}0 & 0 \\ 218 & 334\end{array}$

$319 \quad 200$

$0 \quad 15$

$\begin{array}{rr}0 & 0 \\ 279 & 275\end{array}$

$122 \quad 105$

$537 \quad 522$

522
76

0

$$
0
$$$$
0
$$$$
0
$$$$
0
$$$$
0
$$$$
0
$$

1359

53

881

1015

1

3

0

0

24

20

0

176

296

0

122
0

3

0

17

132

24

209

0

0

71

9

0

24

7251

0

0

9

195

120

0

0

252

235

527

1

102

26

0

0

0

0

0

705

161

971

0

4

0

0

0

153

0

555

64

0

81
638

3

0

17

379

25

162

472

0

56

226

0

0
10234

0234
0

0

0

208

250

0

0

252

243

533

0

158

141

2

0

0

109

0

1897

79

979

0

4

138

0

0

162

87

515

227

245

180
1630

3

0

1

126

23

161

279

186

78

80

0

0

0

0

0
223

145

214

133

252

228

533

0

154

121

0

168

873

0

30

1744

51

1496

0

4

78

39

0

74

0

458

84

24

187 
Page - 56

PURCHASERS OF SHORT-TERM GAS

Estimated Volumes Imported (MMCF)
1993

Oct.-Dec.
1993

Jan.-Mar.

Apr.-June

1993

July-Sep.
1993

Oct.-Dec.

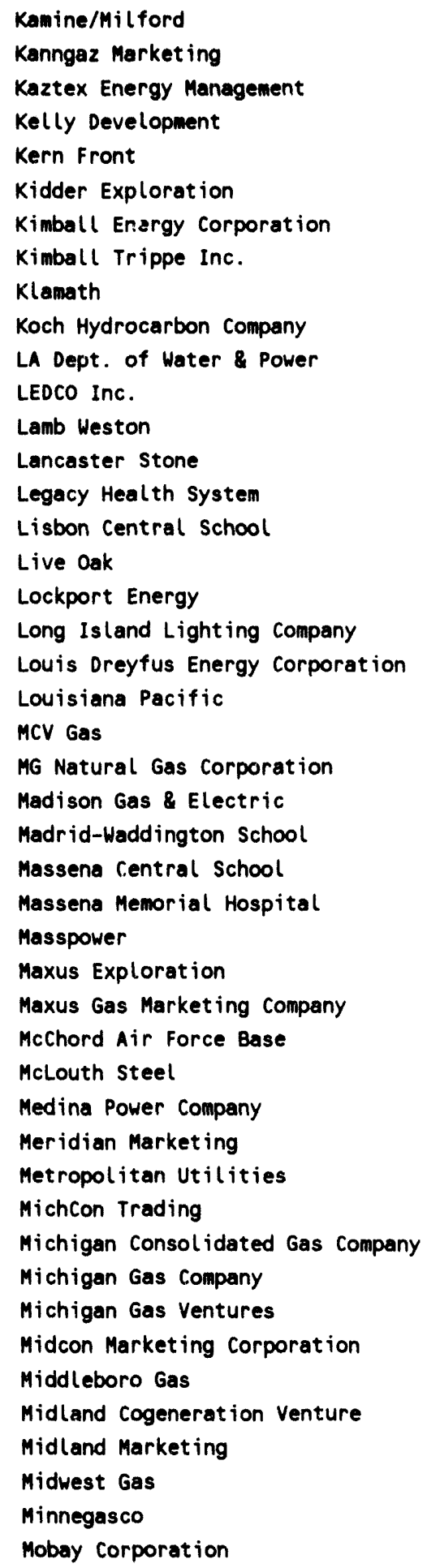

$$
27
$$$$
0
$$$$
553
$$$$
0
$$

9

0

149

20

2

0

130

9

0

0

0

$\begin{array}{rr}4 & \\ 0 & \\ 386 & 138 \\ 0 & \\ 0 & \\ 2 & \end{array}$

\section{0}

$0 \quad 0$

$0 \quad 0$

$195 \quad 545$

138

0

20

5

69

0

0

23

187

101

0

0

0

0

3633

44

223

21

0

2

5

4

451

0

179

0

0

7875

2233

578

0

0
0

363

0

1168

4806

6
0
0
45
0

0

0

960

114

0

0

0

0

92

0

42

2

0

0

828

1427

61

0

0

60

2

6

4

0

0

0

0

0

164

259

666

0

0

726

585

0

0

117

287

1158

1952

0 
Page - 57

\section{PURCHASERS OF SHORT-TERM GAS \\ Estimated Volumes Imported (MMCF)}

$\begin{array}{ccccc}1992 & 1993 & 1993 & 1993 & 1993 \\ \text { Oct.-Dec. } & \text { Jan.-Mar. } & \text { Apr.-June } & \text { July-Sep. } & \text { Oct.-Dec. }\end{array}$

\section{oct.}

Mobil Natural Gas Inc.

Mock Resources

Mountain Front

Mountain Fuel

Mountain Gas Resources

Murphy Gas Gathering

N.Y. State Electric \& Gas

National Fuel Gas Distribution

National Fuel Gas Supply Corporation

National fuel Resources

National Gas Resources

National Steel

Natural Gas Clearinghouse

Natural Gas Pipeline

Natural Gas Resources

Naval Air Station

Neste oy

Neste Trading USA Inc.

New England Power Company

New York Power Authority

$\mathrm{Ni}$-Tex

Niagara Mohawk

Nipsco Energy Trading Corporation

Norfolk Paper Company

Nortech Energy Corporation

North American Resources Company

North Canadian Marketing Corp.

North East Heat \& Light

Northern Consolidated Power Inc.

Northern Illinois Gas

Northern Indiana Gas Company

Northern Natural Gas

Northern States Power (MN)

Northern States Power (WI)

Northern States Power Company

Northern Utilities, Inc.

Northridge Petroleum Marketing U.S., Inc.

Northwest Alloys

Northwest Aluminum Company

Northwest Natural Gas Company

Northwest Pipeline
Northern Minnesota Utilities

$\begin{array}{lrr}\text { Northwest Pipeline Fuel } & 0 & 0 \\ \text { Northwestern Public Service Company } & 128 & 162 \\ \text { Norwood School Board } & 0 & 0 \\ \text { Norwood-Norfolk School } & 0 & 2\end{array}$

$\begin{array}{lrr}\text { Northwest Pipeline Fuel } & 0 & 0 \\ \text { Northwestern Public Service Company } & 128 & 162 \\ \text { Norwood School Board } & 0 & 0 \\ \text { Norwood-Norfolk School } & 0 & 2\end{array}$

$\begin{array}{lrr}\text { Northwest Pipeline Fuel } & 0 & 0 \\ \text { Northwestern Public Service Company } & 128 & 162 \\ \text { Norwood School Board } & 0 & 0 \\ \text { Norwood-Norfolk School } & 0 & 2\end{array}$

$\begin{array}{lrr}\text { Northwest Pipeline Fuel } & 0 & 0 \\ \text { Northwestern Public Service Company } & 128 & 162 \\ \text { Norwood School Board } & 0 & 0 \\ \text { Norwood-Norfolk School } & 0 & 2\end{array}$

68

$\begin{array}{rr}3 & \\ 682 & 514 \\ 0 & 64 \\ 0 & \\ 0 & 119 \\ 0 & \\ 0 & \\ 0 & \end{array}$

44

514

64

0

119

0

0

115

503

$\begin{array}{rr}0 & 503 \\ 167 & 99\end{array}$

1550

9427

505

0

0

0

0

0

98

0
3928

222

39

0

453

1740

0

133

4618

1935

2253

0

0

0

0

0

0

0

38

0

744

62

0

1008

919

0

686

5042

1460

1190

1580

628

0

49

0

161

0

0

1733

3229 $\begin{array}{lrr}\text { Northwest Pipeline Fuel } & 0 & 0 \\ \text { Northwestern Public Service Company } & 128 & 162 \\ \text { Norwood School Board } & 0 & 0 \\ \text { Norwood-Norfolk School } & 0 & 2\end{array}$

$\begin{array}{lrr}\text { Northwest Pipeline Fuel } & 0 & 0 \\ \text { Northwestern Public Service Company } & 128 & 162 \\ \text { Norwood School Board } & 0 & 0 \\ \text { Norwood-Norfolk School } & 0 & 2\end{array}$

$\begin{array}{lrr}\text { Northwest Pipeline Fuel } & 0 & 0 \\ \text { Northwestern Public Service Company } & 128 & 162 \\ \text { Norwood School Board } & 0 & 0 \\ \text { Norwood-Norfolk School } & 0 & 2\end{array}$

0

396

0

164

0

0

0

0

0

35

123

1808

1117

2782

0

0

21

0

0

36

0

0
259

62

0

1706

3537

0

381

5682

0

1033

0

0

0
372

372

68

0

89

1664

2723
July-Sep. Oct.-Dec.

$36 \quad 2756$

$843 \quad 1089$

$0 \quad 0$

$0 \quad 0$

350

$0 \quad 198$

$0 \quad 763$

$0 \quad 319$

$0 \quad 23$

$292 \quad 678$

$177 \quad 489$

$1659 \quad 1714$

$1205 \quad 4226$

$500 \quad 0$

$8 \quad 43$

$0 \quad 1$

$0 \quad 1338$

$86 \quad 0$

$0 \quad 363$

$0 \quad 0$

$0 \quad 0$

$456 \quad 314$

$63 \quad 59$

1560

$1623 \quad 297$

$1973 \quad 2271$

$0 \quad 86$

$282 \quad 265$

105427733

$0 \quad 0$

$648 \quad 1880$

$0 \quad 330$

$302 \quad 1430$

$0 \quad 204$

$554 \quad 223$

$0 \quad 58$

$128 \quad 88$

$0 \quad 158$

$82 \quad 105$

$1391 \quad 1733$

$2704 \quad 2736$

$0 \quad 1$

$192 \quad 129$

22 
Page - 58

\section{PURCHASERS OF SHORT-TERM GAS \\ Estimated Volumes Imported (MMCF)}

\begin{tabular}{|c|c|c|c|c|c|}
\hline & $\begin{array}{c}1992 \\
\text { Oct.-Dec. }\end{array}$ & $\begin{array}{c}1993 \\
\text { Jan. - Mar. }\end{array}$ & $\begin{array}{c}1993 \\
\text { Apr. - June }\end{array}$ & $\begin{array}{c}1993 \\
\text { July-Sep. }\end{array}$ & $\begin{array}{c}1993 \\
\text { Oct. - Dec. }\end{array}$ \\
\hline O \& R Energy & 268 & 330 & 298 & 569 & 1606 \\
\hline occidental & 88 & 43 & 28 & 0 & 0 \\
\hline Ocean State Power & 61 & 12 & 0 & 0 & 0 \\
\hline Olympic Fuels, Inc. & 1049 & 497 & 1376 & 1169 & 1091 \\
\hline Omaha Utilities District & 0 & 0 & 0 & 63 & 0 \\
\hline Orange \& Rockland Marketing & 425 & 22 & 176 & 0 & 0 \\
\hline Orange \& Rockland Utilities & 2701 & 2468 & 2264 & 2457 & 2289 \\
\hline Orchard Gas & 0 & 0 & 10 & 0 & 0 \\
\hline Oregon Natural Gas Development & 1260 & 574 & 268 & 535 & 79 \\
\hline Oroweat & 0 & 0 & 0 & 0 & 9 \\
\hline Oryx Gas Marketing Company & 0 & 0 & 0 & 0 & 86 \\
\hline oxy, 1nc. & 0 & 28 & 0 & 0 & 0 \\
\hline PACCO & 0 & 147 & 281 & 50 & 94 \\
\hline PEMEX & 404 & 122 & 0 & 0 & 0 \\
\hline PG\&E Exchange & 0 & 0 & 0 & 0 & 284 \\
\hline PG\&E Gas Supply Business Unit- & 0 & 0 & 0 & 0 & 35124 \\
\hline PG\&E UEG & 0 & 0 & 0 & 0 & 1134 \\
\hline Pacific Coastal Gas & 0 & 0 & 0 & 0 & 10 \\
\hline Pacific Gas \& Electric Company & 2009 & 1512 & 635 & 2198 & 6155 \\
\hline Pacific Gas Transmission & 2255 & 1825 & 74 & 3203 & 859 \\
\hline Pacific Gas Transmission (Linepack) & 0 & 0 & 0 & 0 & 146 \\
\hline Pacific Gas Transmission Fuel & 0 & 0 & 0 & 0 & 11 \\
\hline Paladin & 1290 & 1375 & 1136 & 983 & 327 \\
\hline Pan-Alberta & 60 & 0 & 0 & 0 & 0 \\
\hline Pancanadian Petroleum Ltd. & 0 & 0 & 0 & 171 & 410 \\
\hline Panda Resources & 494 & 271 & 0 & 0 & 0 \\
\hline Panhandle Eastern Pipe Line Company & 0 & 0 & 0 & 0 & 138 \\
\hline Panhandle Trading Company & 209 & 2137 & 111 & 164 & 441 \\
\hline Paragon Gas Corporation & 573 & 0 & 0 & 0 & 0 \\
\hline Pawtucket Power Associates & 0 & 55 & 337 & 0 & 43 \\
\hline Peabody Municipal Light Plant & 0 & 4 & 1 & 0 & 0 \\
\hline Peoples Gas \& Light & 0 & 0 & 74 & 72 & 0 \\
\hline Peoples Natural Gas Company & 728 & 1815 & 1430 & 1964 & 1851 \\
\hline Pepperell Power Associates, L.P. & 39 & 122 & 145 & 141 & 43 \\
\hline Petro Canada Hydrocarbons & 1318 & 1341 & 1365 & 1379 & 1410 \\
\hline Pfizer Inc. & 50 & 0 & 0 & 0 & 0 \\
\hline Phibro Oil \& Gas Inc. & 0 & 0 & 0 & 314 & 920 \\
\hline Philadelphia Electric Colipany & 0 & 98 & 0 & 0 & 48 \\
\hline Phoenix Chemical & 422 & 96 & 0 & 153 & 158 \\
\hline Piedmont Natural Gas Company & 331 & 296 & 0 & 0 & 0 \\
\hline Pilgrim & 0 & 0 & 0 & 8 & 34 \\
\hline Pioneer Gas Investments & 22 & 33 & 73 & 0 & 0 \\
\hline Pipeline Storage & 0 & 0 & 90 & 0 & 0 \\
\hline Plum Creek Manufacturing & 43 & 52 & 30 & 100 & 59 \\
\hline Poco Petroleum & 0 & 14 & 0 & 0 & 211 \\
\hline Polaris & 0 & 0 & 0 & 156 & 0 \\
\hline
\end{tabular}


Page - 59

PURCHASERS OF SHORT-TERM GAS

Estimated Volumes Imported (MMCF)

1992

Jan. - Mar.

Portland General Electric Company

Potlatch

Potsdan Central School

Potsdam college

Potsdam Hardwoods

Potsdam Laundry

Potters Industries

Power City Partners, L.P.

Prairieland Energy Marketing

Premier Edible Oils

Premier Enterprises

Prior Energy Corporation

Public Service Gas \& Electric

Public Service of Colorado

Public Service of North Carolina

Puget Sound Power \& Light

Questar Pipeline Co.

Rangel ine Corporation

Redwood Resources Inc.

Rhone-Poulenc

Rochester Gas \& Electric Company

Rochester Psychiatric Institute

Rockland Psychiatric

Roseburg Lumber

SUNY - Farmingdale

SUNY - N.Y.

SUNY - Old Westbury

SUNY - Stonybrook

Salmon Resources

San Diego Gas \& Electric Company

San Joaquin

Santanna Natural Gas

Scanna Hydrocarbons

Scott Paper

Seagull Energy

Seattle Steam

Selkirk Cogeneration Partners

Semco Energy Services, Inc.

Shell oil company

Shoreline

Sierra Pacific Power Company

Signal fuels Trading Corporation

Simpson Paper

Simpson Tacoma Kraft Company

Sioux Pointe Inc.

sithe Energy
Oct.-Dec.

\section{0}

238

3

0

0

0

33

53

305

0

98

0

244

171

0

391

0

37

521

126

10

7

0

0

0

0

0

0

215

28

30

1588

0

0

0

436

0

0

287

0

3652

2

189

647

1836

0
1993

1993

Apr.-June

1281

340

5

46

2

1

53

0

61

0

0

0

244

389

343

1885

14

0

0

133

156

0

$$
0
$$

9

$$
0
$$$$
0
$$

0

0

0

0

0

1814

0

0

0

500

18

101

102

0

3134

0

0

134

2270

0
1993

July-Sep. Oct.-Dec.
$1383 \quad 3081$

323

5

47

2

2

78

$$
0
$$

347

49

0

0

542

402

0

0

454

0

87

38

0

0

12

187

5

0

6

60

456

259

46

355

198

208

56

76

0

0

0

46

3153

0

0

525

2896

0
336

6

47

2

2

54

0

11

53

0

61

728

139

515

15

77

0

751

128

0

0

0

35

0

384

0

0

1874

600

0

63

0

121

0

316

6

0

0

0

4705

0

143

1944

6 
Page - 60

PURCHASERS OF SHORT-TERM GAS

Estimated Volumes Imported (MMCF)

\begin{tabular}{|c|c|c|c|c|c|}
\hline & $\begin{array}{c}1992 \\
\text { Oct.-Dec. }\end{array}$ & $\begin{array}{c}1993 \\
\text { Jan. - Mar. }\end{array}$ & $\begin{array}{c}1993 \\
\text { Apr. - June }\end{array}$ & $\begin{array}{c}1993 \\
\text { July-Sep. }\end{array}$ & $\begin{array}{c}1993 \\
\text { Oct. - Dec. }\end{array}$ \\
\hline Socal Edison Company & 1248 & 0 & 126 & 20 & 0 \\
\hline Southeastern Michigan Gas Company & 246 & 155 & 82 & 0 & 168 \\
\hline Southern California Edison Company & 0 & 0 & 0 & 0 & 3175 \\
\hline Southern California Gas Company & 71 & 0 & 238 & 628 & 216 \\
\hline Southern Connecticut Gas Company & 0 & 1317 & 0 & 0 & 155 \\
\hline Southwest Gas Corporation & 1018 & 1055 & 71 & 366 & 825 \\
\hline St. Laurence Psychiatric & 0 & 84 & 85 & 86 & 29 \\
\hline St. Lawrence University & 21 & 33 & 35 & 49 & 47 \\
\hline St. Regis Nursing Home & 1 & 1 & 1 & 2 & 1 \\
\hline Starret Spring & 0 & 0 & 7 & 139 & 0 \\
\hline State University of New York & 0 & 0 & 2 & 0 & 0 \\
\hline Stella Cheese Company & 11 & 17 & 17 & 17 & 16 \\
\hline Stone container corporation & 466 & 447 & 440 & 356 & 525 \\
\hline Sumas Cogeneration & 0 & 0 & 1094 & 942 & 902 \\
\hline Sunpacific & 862 & 0 & 280 & 9 & 471 \\
\hline Sunrise Energy & 194 & 1320 & 617 & 603 & 2142 \\
\hline T.W. Phillips Gas \& Oil co. & 0 & 0 & 10 & 0 & 0 \\
\hline TASCO & 15 & 0 & 0 & 0 & 0 \\
\hline TEMCO & 0 & 518 & 0 & 0 & 165 \\
\hline TETCO & 0 & 98 & 0 & 0 & 0 \\
\hline TM STAR & 0 & 0 & 767 & 756 & 298 \\
\hline Tarpon Gas Marketing & 0 & 23 & 0 & 113 & 52 \\
\hline Tarrant Resources & 0 & 0 & 0 & 0 & 84 \\
\hline Teledyne & 0 & 0 & 85 & 56 & 70 \\
\hline Tenaska Marketing Ventures & 806 & 1034 & 1733 & 1588 & 3479 \\
\hline Tennessee Gas Pipeline Company & 6970 & 5399 & 5926 & 5633 & 1653 \\
\hline Tenngasco Corporation & 369 & 398 & 146 & 22 & 0 \\
\hline Terra Industries & 608 & 1514 & 1563 & 1953 & 1891 \\
\hline TexEnergy & 0 & 0 & 12 & 0 & 0 \\
\hline Texpar Energy, Inc. & 887 & 676 & 307 & 26 & 0 \\
\hline Texaco Gas Marketing Inc. & 539 & 1012 & 165 & 22 & 0 \\
\hline Texas-ohio Gas, Inc. & 109 & 242 & 280 & 0 & 0 \\
\hline The Brooklyn Union Gas Company & 0 & 5 & 0 & 0 & 0 \\
\hline The Montana Power' mpany & 599 & 1161 & 0 & 0 & 153 \\
\hline Torch Gas L.C. & 0 & 18 & 128 & 82 & 489 \\
\hline Tosco Energy & 0 & 0 & 121 & 0 & 80 \\
\hline Transcontinental Gas Pipeline & 147 & 0 & 0 & 0 & 0 \\
\hline Transenergy & 36 & 0 & 0 & 0 & 0 \\
\hline Transfuel Marketing Company & 0 & 14 & 31 & 9 & 0 \\
\hline Transok & 0 & 0 & 0 & 0 & 146 \\
\hline Transwestern Fuel & 0 & 1 & 0 & 0 & 0 \\
\hline Tristar Gas Marketing & 253 & 69 & 90 & 1266 & 506 \\
\hline Triumph Gas & 128 & 160 & 40 & 0 & 0 \\
\hline U.S. Gypsum & 91 & 258 & 0 & 0 & 0 \\
\hline U.S. Transportation & 9 & 0 & 0 & 0 & 0 \\
\hline Unigas & 1187 & 231 & 279 & 1165 & 468 \\
\hline
\end{tabular}


Page - 61

PURCHASERS OF SHORT-TERM GAS

E'stimated Volumes Imported (MMCF)
1993
Apr.-June July-Sep. Oct.-Dec.

1993

Union Gas Company

Union Gas Limited

Union Oil

Union Pacific Fuels

Union Pacific Resources

United Helpers Management Company

Universal Frozen Foods

Unocal

Utah Gas Services

VA Medical

Valero Industrial Gas

Valley Gas Company

Vanalco

Various MI State Markets

Various NY State Markets

Vermont Gas Systems, Inc.

Victoria Gas corporation

Viking Gas Transmission

Village of Potsdam Housing Authority

Virginia Power

Volunteer Energy

WP Natural Gas

Wainoco Oil

Washington Energy

Washington Natural Gas Company

Washington Water Power Company

Watson Cogeneration

Watsonville cogeneration

Wescana Energy Marketing (U.S.) Inc.

Westar Marketing Company

Western Gas Marketing Inc.

Western Gas Resources

Western Gas Services

Westfield Gas \& Electric Light Dept.

Weyerhauser

Oct. Dec.

Jan. -Mar

Apr. - June

1256

0

1132

Whitman college

Willamette

Williams Gas Marketing

Will iams Natural Gas Company

Williams/Northwest Pipeline Pool

Windward Energy \& Marketing Inc.

Wisconsin Gas

Wisconsin Natural Gas

Wisconsin Power \& Light

Wisconsin Public Service Corp.

Wisconsin Southern
463

3

188

0

98

0

248

2

190

0

3

17

0

171

2

0

0

0

73

156

5703

2230

861

0

0

664

77

244

430

0

269

$\begin{array}{rr}0 & \\ 1286 & \\ 0 & \\ 1259 & \\ 0 & \\ 4 & \\ 146 & \\ 0 & \\ 0 & \\ 0 & \\ 23 & \\ 0 & \\ 0 & \\ 0 & \\ 47 & \end{array}$

$\begin{array}{rrr}0 & 0 & \\ 1383 & 1426 & 1440\end{array}$

$\begin{array}{lll}0 & 8 & 0\end{array}$

$1256 \quad 1317$

0

298

0

588

0

1512

486

45
125

4

183

18

40

0

0

0

0

0

1153

409

11

3

0

0

979

0

42

5395

2598

804

0

0

0

534

486

0

2

67

2

0

28

102

53

4

145

42

17

17

0

0

0

0

0

0

0

0

3

6

29
436

436

440

2051

550

0

0

170

0

3691

157

0

0

291

0

0

0

0

0

303

401

91

1161

730

718

0

\section{5}

440

0

433

901

4

201

24

14

22

133

0

0

429

0

52

0

0

3

211

0

654

0

45

4658

3242

740

271

0

0

351

173

0

0

679

0

5

0

0

0

41

7776

162

772

732 
Page - 62

PURCHASERS OF SHORT-TERY GAS

Estimated Volumes Imported (MMCF)

1992

Oct.-Dec.

0
54
79
0
37

Wyman Gordon

Wyoming Industrial Gas

$x$ Energy, Inc.

Yankee Gas Services

Yuma Gas Corporation

TOTALS
198096
1993

Jan. - Mar.

1993

Apr. - June

0
62
0
0
73

0

0

66

82

50

8

193634

168674
1993

Juty-Sep. Oct. -Dec.

$\begin{array}{rr}45 & 0 \\ 65 & 35 \\ 0 & 0 \\ 0 & 0 \\ 0 & 0\end{array}$

$\underline{251030}$ 


\section{SHORT-TERM SALES}

4th Quarter 1993

Estimated Volumes (MMCF) \& Prices (\$/MMBTU)

$\begin{array}{llll} & & \\ \text { Importer } & \text { Point } & & \\ \text { Seller } & \text { Of } & \text { Octoberterly } & \text { Total } \\ \text { Purchaser/End User } & \text { Entry } & \text { November Pecember Price Vol. Price Vol. Price Vol. Price }\end{array}$

AIG TRAOING CORPORATION

AIG Trading

Coastal Gas Marketing Company

Detroit, Michigan $0 \quad N / A$

$0 \quad N / A$

139

2.22

139

2.22

AIG TRADING CORPORATION

AIG Trading

Coenergy Trading Company

Noyes, Minnesota

$0 \quad N / A$

$18 \quad 2.00$

$0 \quad N / A$

$18 \quad 2.00$

AIG TRADING CORPORATION

AIG Trading

Coenergy Trading Company

St. Clair, MI

$0 \quad$ N/A

$0 \quad N / A$

$80 \quad 2.22$

$80 \quad 2.22$

AIG TRADING CORPORATION

AIG Trading

Direct Energy

Niagara Falls, NY

$0 \quad N / A$

$0 \quad$ N/A

$60 \quad 2.22$

60

2.22

AIG TRADING CORPORATION

AIG Trading

Howard Energy Company

Noyes, Minnesota

$69 \quad 2.24$

O N/A

O N/A

$69 \quad 2.24$

AIG TRADING CORPORATION AIG Trading

Various MI State Markets

St. Clair, MI

$0 \quad N / A$

$0 \quad N / A$

429

2.22

429

2.22

AMERICAN MUNTER EXPLORATION LTD. Alberta \& southern Company Ltd. Jonan Gas Marketing

AMERICAN HUNTER EXPLORATION LTD.

Alberta southern Company Ltd. 


\section{SHORT-TERM SALES \\ 4th Quarter 1993}

Estimated Volumes (MMCF) \& Prices (\$/MMBTU) -- cont.

\begin{tabular}{llll} 
& & & \\
& & \\
Importer & Point & Tuarterly \\
Seller & of & October & November December \\
Purchaser/End User & Entry & Vol. Price & Vol. Price Vol. Price Vol. Price \\
\hline
\end{tabular}

AMERICAN HUNTER EXPLORATION LTD.

Alberta \& Southern Company Ltd.

Natural Gas Resources

Eastport, Idaho

$0 \quad N / A$

10

2.00

O N/A

$10 \quad 2.00$

AMERICAN HUNTER EXPLORATION LTD. Alberta \& Southern Company Ltd. Northwest Pipeline Eastport, Idaho

O N/A

$0 \quad N / A$

143

1.82

143

1.82

AMERICAN HUNTER EXPLORATION LTD. Alberta \& Southern Company Ltd. Pacific Gas \& Electric Company

AMERICAN HUNTER EXPLORATION LTD. Alberta \& Southern Company Ltd.

Pacific Gas Transmission

AMERICAN HUNTER EXPLORATION LTD. Alberta \& Southern Company Ltd. Pacific Gas Transmission Fuel

AMERICAN HUNTER EXPLORATION LTD. Alberta \& Southern Company Ltd. Redwood Resources Inc.

AMERICAN HUNTER EXPLORATION LTD.

B.C. Gas Inc.

Clay Basin Storage 


\section{SHORT-TERM SALES \\ 4th Quarter 1993}

Estimated Volumes (MMCF) \& Prices (S/MMBTU) - cont.

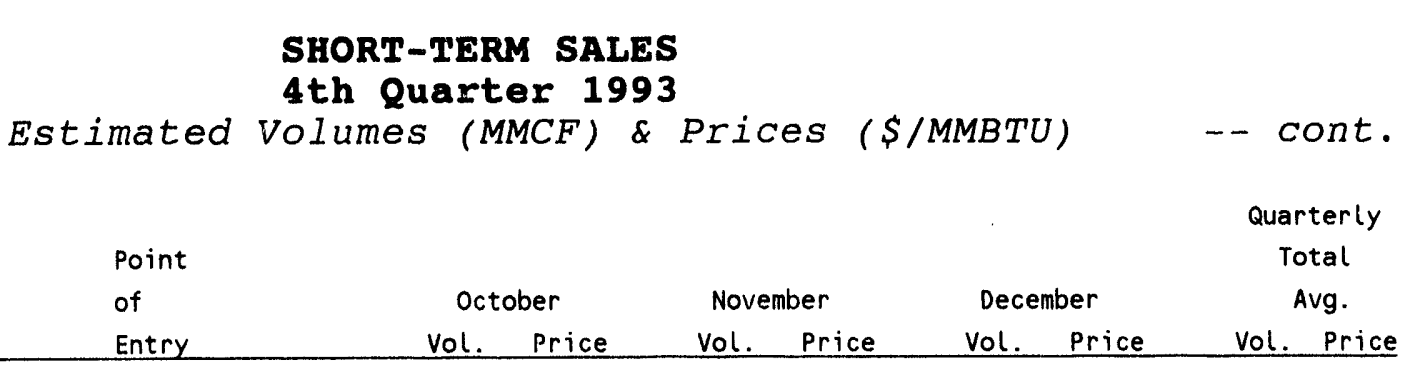

AMERICAN HUNTER EXPLORATION LTD.

B. C. Gas Inc.

Mock Resources

Sumas, Washington $\quad 104 \quad 1.60$

$0 \quad N / A$

44

2.25

$148 \quad 1.79$

AMERICAN HUNTER EXPLORATION LTD.

B.C. Gas Inc.

Northwest Pipeline

Sumas, Washington

$75 \quad 1.60$

26

1.83

$7 \quad 2.25$

108

1.70

AMERICAN HUNTER EXPLORATION LTD.

B.C. Gas Inc.

Northwest Pipeline Fuel

Sumas, Washington

$1 \quad 1.66$

$0 \quad N / A$

O N/A

$1 \quad 1.66$

AMERICAN HUNTER EXPLORATION LTD.

B.C. Gas Inc.

Pacific Gas Transmission

Sumas, Washington

O N/A

$0 \quad N / A$

$1 \quad 2.25$

$1 \quad 2.25$

AMERICAN HUNTER EXPLORATION LTD.

B.C. Gas Inc.

Sierra Pacific Power Company

Sumas, Washington

$0 \quad$ N/A

$246 \quad 1.83$

$0 \quad N / A$

246

1.83

AMOCO CANADA MARKETING CORPORATION Amoco Canada Marketing Corporation Amoco Trading

AMOCO CANADA MARKETING CORPORATION Amoco Canada Marketing Corporation Portland General Electric Compan; 


$$
\text { Page - } 66
$$

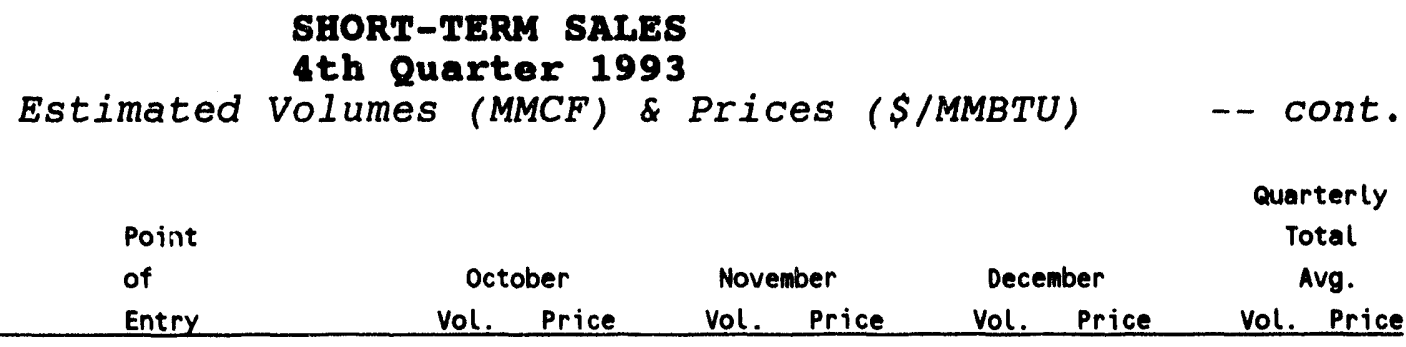

ANR PIPELINE COMPANY Western Gas Marketing Limited ANR Pipeline Company Noyes, Minnesota 1164 2.30 1199

ARCO PRODUCTS COMPANY CanHest Marketing Arco Products Company

ARCO PRODUCTS COMPANY Canadian Hydrocarbons Marketiing Arco Products Company

ARCO PRODUCTS COMPANY Unocal Canada Limited Arco Products Company

BAY STATE GAS COMPANY Renaissance Energy Ltd. Bay State Gas Company

BRIDGEGAS U.S.A. INC. Northstar Energy Corporation Peoples Natural Gas Company

Port of Morgan, MT $12 \quad 1.56$

$3 \quad 1.64$

O N/A

15

1.58

BROAD STREET OIL \& GAS COMPANY Alberta \& Southern Company Ltd. Broad Street Oil \& Gas

Eastport, Idaho 145

1.69

$0 \quad$ N/A

N/A

145

1.69

BROAD STREET OIL \& GAS COMPANY Continental Energy Marketing Ltd. Broad Street Oil \& Gas
Eastport, Idaho

$0 \quad$ N/A

121

1.93

0

N/A

121

1.93 


$$
\text { Page - } 67
$$

\section{SHORT-TERY SALES \\ 4th Quarter 1993}

Estimated Volumes (MMCF) \& Prices (\$/MMBTU) -- cont.

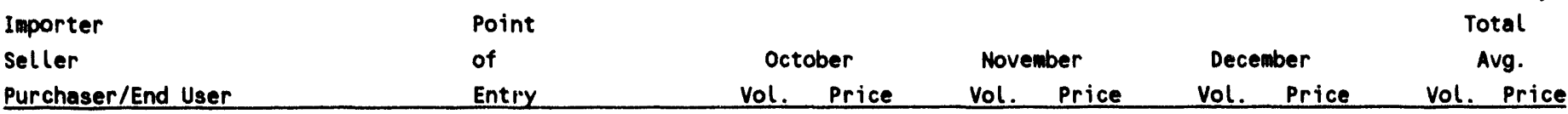

BROAD STREET OIL \& GAS COMPANY

North Canadian Marketing Inc.

Broad Street oil \& Gas

Eastport, Idaho

$0 \quad$ N/A

$0 \quad$ N/A

122

2.33

122

2.33

CANADA IMPERIAL OIL LIMITED Imperial oil Resources

Canada Imperial Oil Limited

Eastport, Idaho

O N/A

$303 \quad 1.81$

305

2.34

608

2.08

CANADIAN HYDROCARBONS MARKETING (U.S.) INC.

Canadian Hydrocarbons Marketing

Arco Products Company

Sumas, Washington

$0 \quad N / A$

O N/A

4

2.35

4

2.35

CANADIAN HYDROCARBONS MARKETING (U.S.) INC.

Canadian Hydrocarbons Marketing

Cascade Natural Gas

Sumas, Washington

$0 \quad$ N/A

289

2.04

230

2.42

519

2.21

CANADIAN HYDROCARBONS MARKETING (U.S.) INC.

Canadian Hydrocarbons Marketing

Kimball Energy Corporation

Sumas, Washington

$0 \quad N / A$

$136 \quad 2.05$

652

2.36

788

CANADIAN HYDROCARBONS MARKETING (U.S.) INC.

Canadian Hydrocarbons Marketing

Mock Resources

Sumas, Washington

$0 \quad N / A$

61

2.21

$0 \quad N / A$

61

2.21

CANADIAN HYDROCARBONS MARKETING (U.S.) INC.

Canadian Hydrocarbons Marketing Paladin

Babb, Montana

$217 \quad 2.02$

78

2.29

$32 \quad 1.91$

327

2.07

CANADIAN HYDROCARBONS MARKETING (U.S.) INC.

Canadian Hydrocarbons Marketing

sumas Cogeneration

Sumas, Washington

301

1.39

298

1.67

302

1.66

902

1.57 


\section{SHORT-TERM SALES}

4th Quarter 1993

Estimated Volumes (MMCF) \& Prices (S/MMBTU) - cont.

\section{Estimated Volumes (MMCF) \& Prices (S/MMBTU)}

\begin{tabular}{|c|c|c|c|c|c|c|}
\hline Importer & Point & & & & & $\begin{array}{c}\text { Quarterly } \\
\text { Total }\end{array}$ \\
\hline Seller & of & October & November & & ber & Avg. \\
\hline Purchaser/End User & Entry & Vol. Price & Vol. Price & Vol & Price & Vol. Price \\
\hline
\end{tabular}

CANADIAN HYDROCARBONS MARKETING (U.S.) INC.

Canadian Hydrocarbons Marketing

Washington Natural Gas Company

Sumas, washington $\quad 0 \quad$ N/A

$49 \quad 3.25$

$49 \quad 3.91$

97

3.58

CANWEST GAS SUPPLY U.S.A., INC.

CanWest Gas Supply Inc.

American Hunter Energy

Sumas, Washington

$542 \quad 1.13$

136

1.39

O N/A

$678 \quad 1.18$

CANWEST GAS SUPPLY U.S.A., INC. CanWest Gas Supply Inc.

BP Oil Company

Sumas, Washington

$45 \quad 1.13$

$0 \quad N / A$

$0 \quad N / A$

45

1.13

CANWEST GAS SUPPLY U.S.A., INC. CanWest Gas Supply Inc.

Boeing Company

Sumas, Washington

$0 \quad N / A$

$52 \quad 1.39$

104

1.82

156

1.68

CANWEST GAS SUPPLY U.S.A., INC.

CanWest Gas Supply Inc.

Bridgegas

Sumas, Washington

$433 \quad 1.13$

$65 \quad 1.39$

- N/A

498

1.16

CANWEST GAS SUPPLY U.S.A., INC.

CanWest Gas Supply Inc.

Coastal Gas Marketing Company

Sumas, Washington

$954 \quad 1.13$

$176 \quad 1.39$

$66 \quad 1.82$

1196

1.21

CANWEST GAS SUPPLY U.S.A., INC.

CanWest Gas Supply Inc.

Destec

Sumas, Washington

$0 \quad$ N/A

O N/A

$82 \quad 1.82$

82

1.82

CANWEST GAS SUPPLY U.S.A., INC.

CanWest Gas Supply Inc.

Development Associates

Sum:s, Washington

$465 \quad 1.13$

O N/A

$87 \quad 1.82$

551

1.24 


$$
\text { Page - } 69
$$

\section{SHORT-TERM SALES \\ 4th Quarter 1993 \\ Estimated Volumes (MMCF) \& Prices (\$/MMBTU) - cont.}

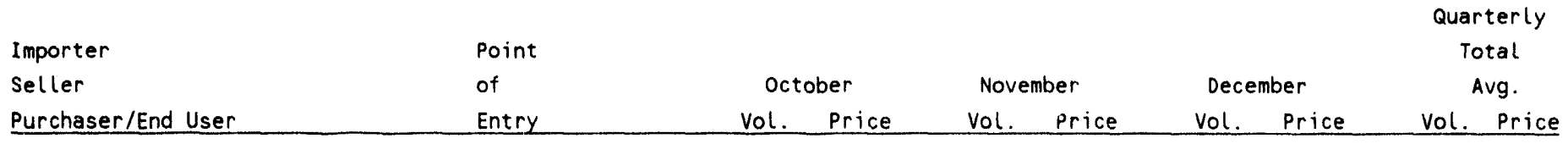

CANWEST GAS SUPPLY U.S.A., INC.

CanWest Gas Supply Inc.

Dexel

Sumas, Washington

$74 \quad 1.13$

148

1.39

$64 \quad 1.82$

$286 \quad 1.42$

CANWEST GAS SUPPLY U.S.A., INC. CanWest Gas Supply Inc.

Ensearch Development Corporation

Sumas, Washington

$245 \quad 1.13$

$0 \quad N / A$

$0 \quad N / A$

$245 \quad 1.13$

CANWEST GAS SUPPLY U.S.A., INC.

CanWest Gas Supply Inc.

Entrade Corporation

Sumas, Washington

O N/A

$2 \quad 1.39$

O N/A

$2 \quad 1.39$

CANWEST GAS SUPPLY U.S.A., INC. CanWest Gas Supply Inc.

Gilroy Energy Company

Sumas, Washington $\quad 0 \quad N / A$

Sumas, Washington

149

1.13

$0 \quad N / A$

$14 ?$

1. 8?

290

$1.4 ?$

CANWEST GAS SUPPLY U.S.A., INC.

CanWest Gas Supply Inc.

IGI Resources

CANWEST GAS SUPPLY U.S.A., INC

CanWest Gas Supply Inc.

J. Aaron Company

CANWEST GAS SUPPLY U.S.A., INC.

CanWest Gas Supply Inc.

Kimball Energy Corporation
Sumas, Washington

$638 \quad 1.13$

$453 \quad 1.39$

$294 \quad 1.82$

1385

1.36

Sumas, Washington

291

1.13

$0 \quad N / A$

C

$N / A$

291

1.13
Sumas, Washington

0

$N / A$

$\begin{array}{ll}72 & 1.39\end{array}$

42. 1.82

114

1.55 


\section{SHORT-TERY SALES \\ 4th Quarter 1993 \\ Estimated Volumes (MMCF) \& Prices (\$/MMBTU) -- cont.}

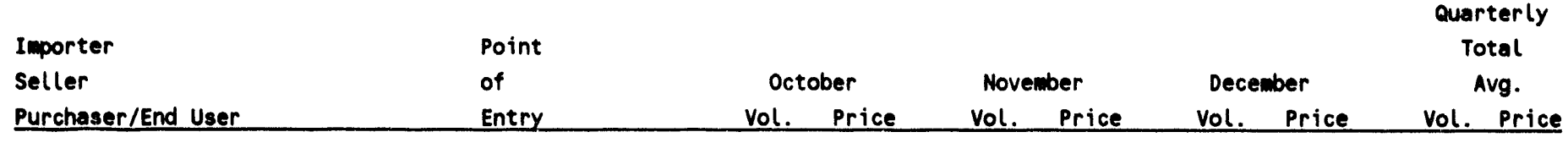

CANUEST GAS SUPPLY U.S.A., INC.

CanWest Gas Supply Inc.

Mock Resources

Sumas, Washington

$28 \quad 1.13$

$0 \quad N / A$

$53 \quad 1.82$

$81 \quad 1.58$

CANUEST GAS SUPPLY U.S.A., INC.

Cantlest Gas Supply Inc.

Natural Gas Clearinghouse

Sumas, Washington

10

1.13

2781.3

$298 \quad 1.82$

587

1.60

CANWEST GAS SUPPLY U.S.A., INC.

CanWest Gas Supply Inc.

Pacific Gas \& Electric Company

Sumas, Washington

O N/A

O N/A

$276 \quad 1.82$

276

1.82

CANWEST GAS SUPPLY U.S.A., INC.

CanWest Gas Supply Inc.

Portland General Electric Company

Sumas, Washington

$0 \quad$ N/A

$192 \quad 1.39$

$652 \quad 1.82$

844

1.72

CANGEST GAS SUPPLY U.S.A., INC.

Cantiest Gas Supply Inc.

Sierra Pacific Power Company

Sumsis, Washington

19

1.13

O N/A

O N/A

19

1.13

CANWEST GAS SUPPLY U.S.A., INC. CanWest Gas Supply Inc.

Sunpacific

CANWEST GAS SUPPLY U.S.A., INC.

CanWest Gas Supply Inc.

TM STAR

Sumas, Washington

298

1.13

$0 \quad$ N/A

O N/A

298

1.13

CANWEST GAS SUPPLY U.S.A., INC.

CanWest Gas Supply Inc.

Washington Natural Gas Company

Sumas, Washington

$0 \quad N / A$

$866 \quad 1.39$

895

1.82

1762

1.61 


\section{SHORT-TERM SALES}

4th Quarter 1993

Estimated Volumes (MMCF) \& Prices ( $\$ / M M B T U$ ) -- cont.

Quarterly

Total

Importer Point

Seller of

Purchaser/End User

of

October

November

Avg.

Vol. Price

Vol. Price

Vol Price

Vol. Price

CANWEST GAS SUPPLY U.S.A., INC.

CanWest Gas Supply Inc.

Washington Water Power Company

Sumas, Washington

O N/A

$577 \quad 1.39$

$684 \quad 1.82$

$1261 \quad 1.62$

CANWEST GAS SUPPLY U.S.A., INC. CanWest Gas Supply Inc.

Watson cogeneration

Sumas, Washington

182

1.13

260

1.39

298

1.82

740

1.50

CASCADE NATURAL GAS CORPORATION Canadian Hydrocarbons Marketing Cascade Natural Gas

Sumas, Washington

$152 \quad 1.80$

$292 \quad 2.04$

233

2.42

677

2.12

CASCADE NATURAL GAS CORPORATION Coastal Gas Marketing Company

Cascade Natural Gas

Sumas, Washington

$79 \quad 1.74$

O N/A

$0 \quad$ N/A

$79 \quad 9.74$

CASCADE NATURAL GAS CORPORATION Grand Valley Gas Canada Ltd.

Cascade Natural Gas

Sumas, Washington

$299 \quad 1.75$

72

1.80

$0 \quad N / A$

$371 \quad 1.76$

CASCADE NATURAL GAS CORPORATION

IGI Resources

Cascade Natural Gas

Eastport, Idaho

O N/A

206

2.24

220

2.24

426

2.24

CASCADE NATURAL GAS CORPORATION Inland Natural Gas

Cascade Natural ias

Sumas, Washington

$29 \quad 1.65$

$0 \quad$ N/A

22

2.05

$51 \quad 1.82$

CASCADE NATURAL GAS CORPORATION Mobil Oil Canada Ltd.

Cascade Natural Gas 


\section{SHORT-TERM SALES}

4th Quarter 1993

Estimated Volumes (MMCF) \& Prices (\$/MMBTU) -- cont.

Quarterly

Total

\begin{tabular}{|c|c|c|c|c|}
\hline Importer & Point & & & Total \\
\hline Seller & of & October & November & December \\
\hline
\end{tabular}

Purchaser/End User Entry

vol. Price

Vol. Price

Vol. Price

Vol. Price

CASCADE NATURAL GAS CORPORATION

Mock Resources, Inc.

Cascade Natural Gas

Sumas, Washington

$20 \quad 1.65$

O N/A

$0 \quad$ N/A

20

1.65

CASCADE NATURAL GAS CORPORATION

Natural Gas Clearinghouse

Cascade Natural Gas

Sumas, Washington $\quad 135 \quad 1.76$

$80 \quad 1.81$

$59 \quad 2.33$

$273 \quad 1.90$

CHEVRON NATURAL GAS SERVICES INC.

Chevron Canada

Chevron Natural Gas Services, Inc.

Eastport, Idaho

$0 \quad N / A$

2346

1.66

2061

2.01

4407

1.82

CIBOLA CORPORATION

Cibola Canada

Cibola corporation

Port of Morgan, MT $148 \quad 1.52$

$0 \quad N / A$

$0 \quad$ N/A

148

1.52

CMEX ENERGY, INC.

PEMEX

Amoco Trading

Hidalgo, Texas

$0 \quad N / A$

$0 \quad N / A$

725

1.88

725

1.88

CNG PRODUCING COMPANY

PEMEX

CNG Producing Company

Hidalgo, Texas

O N/A

$0 \quad$ N/A

235

2.05

235

2.05

COASTAL GAS MARKETING COMPANY

AIG Trading

Panhandle Eastern Pipe Line Company Detroit, Michigan

$0 \quad N / A$

$0 \quad N / A$

138

2.82

138

2.82

COASTAL GAS MARKETING COMPANY

Mobil Oil Canada Ltd.

Northwest Pipeline

Sumas, Washington

O N/A

$0 \quad$ N/A

389

2.26

389

2.26 


$$
\text { Page - } 73
$$

\section{SHORT-TERM SALES \\ 4th Quarter 1993}

Estimated Volumes (MMCF) \& Prices (\$/MMBTU) -- cont.

$\begin{array}{llll}\text { Importer } & \text { Point } & & \\ \text { Seller } & \text { of } & \text { October } & \text { November }\end{array}$

Purchaser/End User

Entry

Vol. Price

Vol. Price

Vol. Price

Vol. Price

COASTAL GAS MARKETING COMPANY Mobil Oil Canada Ltd.

Pacific Gas Transmission

Eastport, Idaho

$0 \quad N / A$

$167 \quad 1.82$

$267 \quad 1.91$

434

1.88

COASTAL GAS MARKETING COMPANY Mobil Oil Canada Ltd.

Poco Petroleum

Sumas, Washington $211 \quad 9.61$

$0 \quad N / A$

$0 \quad$ N/A

211

1.61

COASTAL GAS MARKETING COMPANY Poco Petroleum Ltd.

Pacific Gas Transmission

Eastport, Idaho

$0 \quad$ N/A

$0 \quad N / A$

$60 \quad 1.95$

60

1.95

COASTAL GAS MARKETING COMPANY Tarpon Gas Marketing Ltd.

Blue Lake Storage

St. Clair, MI

116

2.28

$0 \quad N / A$

$0 \quad N / A$

116

2.28

COASTAL GAS MARKETING CGMPANY Tarpon Gas Marketing Ltd.

Gaz Metropolitan

St. Clair, MI

$20 \quad 2.28$

$0 \quad$ N/A

$0 \quad N / A$

20

2.28

COASTAL GAS MARKETING COMPANY Unigas Energy, Inc.

Northern Natural Gas

Eastport, Idaho

$0 \quad N / A$

$0 \quad N / A$

162

2.82

162

2.82

COASTAL GAS MARKETING COMPANY Unigas Energy, Inc.

Northern Natural Gas

Port of Morgan, MT $0 \quad$ N/A 158

$0 \quad N / A$

158

2.29

COENERGY TRADING COMPANY

American International Group Coenergy Trading Company

St. Clair, MI

$0 \quad$ N/A

$0 \quad N / A$

$80 \quad 1.90$

80

1.90 


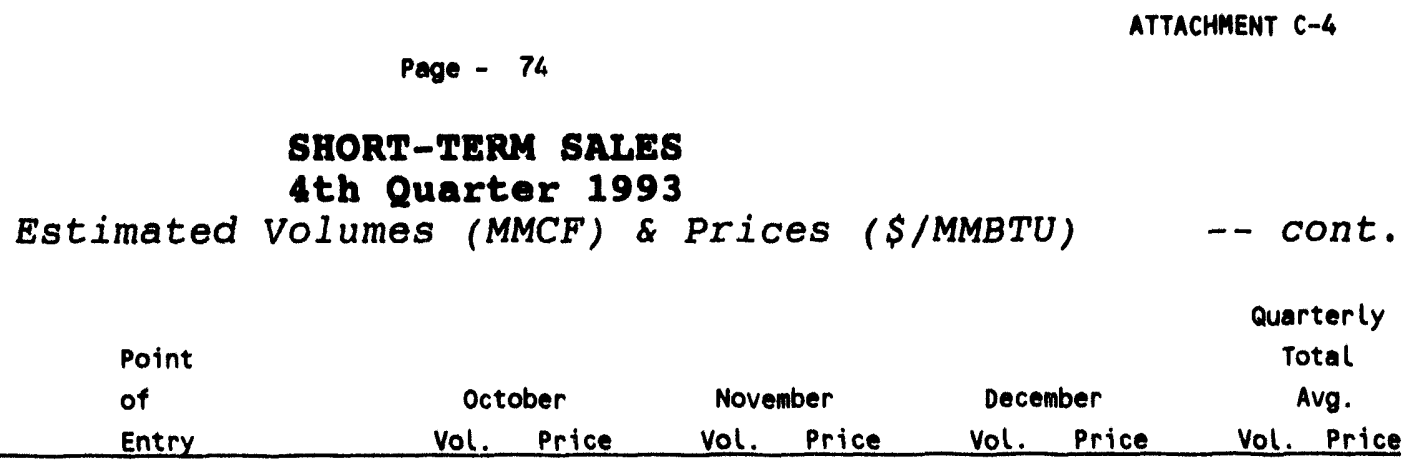

COENERGY TRADING COMPANY

Gaz Metropolitan, Inc.

Coenergy Trading Company

St. Clair, MI

$404 \quad 1.90$

O N/A

$0 \quad$ N/A

404

1.90

CONOCO, INC.

PEMEX

Conoco Inc.

Hidalgo, Texas

O N/A

$0 \quad$ N/A

$389 \quad 1.91$

389

1.91

CONTINENTAL ENERGY MARKETING LTD.

Chevron Canada

Pilgrim

Waddington, NY

$0 \quad$ N/A

34

2.30

$0 \quad N / A$

34

2.30

CONTINENTAL. ENERGY MARKETING LTD. Continental Energy Marketing Ltd. Central Hudson Gas \& Electric

Waddington, NY

$10 \quad 1.96$

O N/A

O N/A

$10 \quad 1.96$

CONTINENTAL ENERGY MARKETING LTD. Cont inental Energy Marketing Ltd. Clarkson University

Massena, New York $\quad \begin{array}{llll}13 & 1.98 & 13 & 2.22\end{array}$

13

2.18

40

2.13

CONTINENTAL ENERGY MARKETING LTD. Continental Energy Marketing Ltd. college of Technology

Massena, New York $\quad 7 \quad 1.98$

Waddington, NY

Continental Energy Marketing Ltd.

Consolidated Edison

CONTINENTAL ENERGY MARKETING LTD. Cont inental Energy Marketing Ltd. Cornell Linen

Massena, New York

0

N/A

$0 \quad$ N/A

65

2.28

65

2.28

.13 


$$
\text { Page - } 75
$$

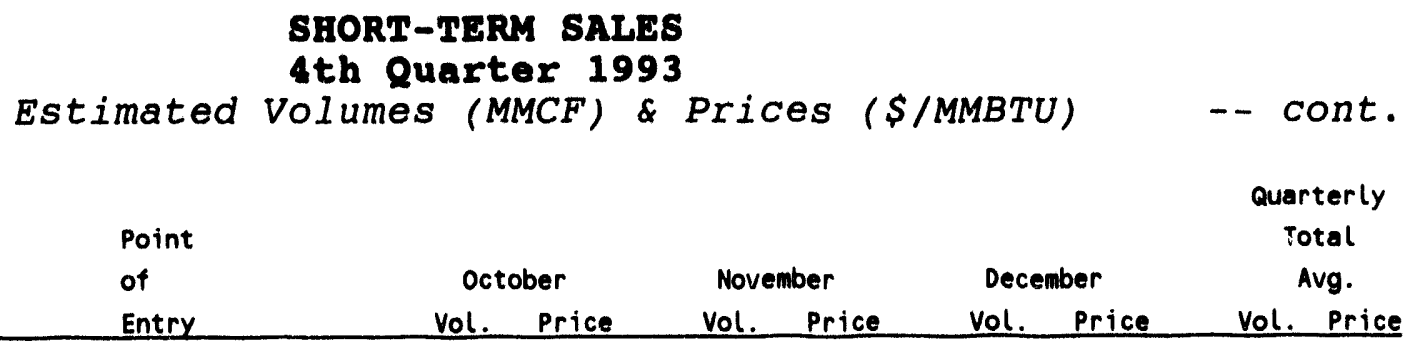

CONTINENTAL ENERGY MARKETING LTD. Cont inental Energy Marketing Ltd. Empire Natural Gas Corporation

CONTINENTAL ENERGY MARKETING LTD. Continental Energy Marketing Ltd. Heuvelton Central School

$\begin{array}{lllllllll}\text { Massena, New York } & 1 & 1.98 & 1 & 2.22 & 1 & 2.18 & 3 & 2.13\end{array}$

CONTINENTAL ENERGY MARKETING LTD. Cont inental Energy Marketing Ltd. Lisbon Central School

Massena, New York 1

1

2.18

CONTINENTAL ENERGY MARKETING LTD. Continental Energy Marketing Ltd. Madrid-Waddington School

Massena, New York $1,1.98$

$1 \quad 2.22$

2.18

2

2.13

CONTINENTAL ENERGY MARKETING LTO. Continental Energy Marketing Ltd. Massena Central School

Massena, New York 21.98

$2 \quad 2.22$

2

2.18

CONTINENTAL ENERGY MARKETING LTD. Continental Energy Marketing Ltd. Massena Memorial Hospital

CONTINENTAL ENERGY MARKETING LTD. Continental Energy Marketing Ltd. North East Heat \& Light 


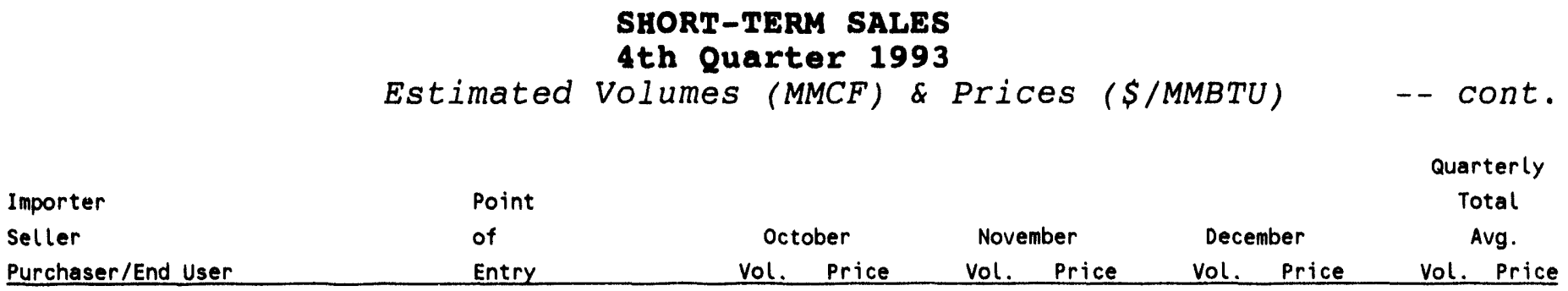

CONTINENTAL ENERGY MARKETING LTD. Cont inental Energy Marketing Ltd. Potsdam Central School

Massena, New York 0 N/A

CONTINENTAL ENERGY MARKETING LTD. Cont inental Energy Marketing Ltd. Potsdan college

CONTINENTAL ENERGY MARKETING LTD. Continental Energy Marketing Ltd. Potsdam Hardwoods

CONTINENTAL ENERGY MARKETING LTD. Cont inental Energy Marketing Ltd. Potsdam Laundry

Massena, New York

$1 \quad 1.98$

$6 \quad 1.87$

$0 \quad N / A$

0

N/A

6

1.87

CONTINENTAL ENERGY MARKETING LTD. Cont inental Energy Marketing Ltd. Sithe Energy

Waddington, NY

$6 \quad 2.10$

$0 \quad$ N/A

$0 \quad N / A$

6

2.10

CONTINENTAL ENERGY MARKETING LTD. Continental Energy Marketing Ltd. St. Lawrence Psychiatric

Massena, New York 29

1.98

O N/A

$0 \quad N / A$

29

1.98

CONTINENTAL ENERGY MARKETING LTD. Direct Energy Marketing Limited Brooklyn Interstate Natural Gas
Waddington, NY

360

1.87

0

N/A

0

N/A

360

1.87 


$$
\text { Page - } 77
$$

\section{SHORT-TERM SALES \\ 4th Quarter 1993}

Estimated Volumes (MMCF) \& Prices (\$/MMBTU) - cont.

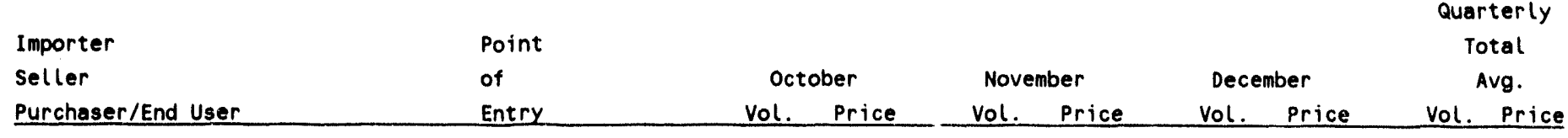

CONTINENTAL ENERGY MARKETING LTD.

Shell Canada Limited

CNF Transmission

Waddington, NY

$0 \quad$ N/A

$64 \quad 2.23$

O N/A

64

2.23

CONTINENTAL ENERGY MARKETING LTO.

Shell Canada Limited

CNG Gas Services

Waddington, NY

$0 \quad N / A$

$12 \quad 2.23$

O N/A

12

2.23

CONTINENTAL ENERGY MARKETING LTD.

Shell Canada Limited

CNG Transmission Corporation

Waddington, NY

$0 \quad N / A$

$0 \quad$ N/A

356

2.54

356

2.54

CONTINENTAL ENERGY MARKETING LTD.

Shell Canada Limited

Consolidated Edison

Waddington, NY

$0 \quad N / A$

$29 \quad 2.23$

O N/A

$29 \quad 2.23$

CONTINENTAL ENERGY MARKETING LTD.

Shell Canada Limited

Long Is land Lighting Company

Waddington, NY

$0 \quad$ N/A

150

2.23

$0 \quad N / A$

150

2.23

CONTINENTAL ENERGY MARKETING LTD.

Shell Canada Limited

TEMCO

Waddington, NY

$0 \quad$ N/A

O N/A

165

2.50

165

2.50

CONTINENTAL ENERGY MARKETING LTD.

Shell Canada Limited

Tarpon Gas Marketing

Waddington, NY

$0 \quad$ N/A

27

2.23

$0 \quad N / A$

$27 \quad 2.23$

CONTINENTAL ENERGY MARKETING LTD.

Shell Canada Limited

Virginia Power

Waddington, NY

O N/A

166

2.23

45

2.30

211

2.24 


$$
\text { Page - } 78
$$

\section{SHORT-TERM SALES}

4th Quarter 1993

Estimated Volumes (MMCF) \& Prices (\$/MMBTU) -- cont.

\begin{tabular}{|c|c|c|c|c|c|}
\hline Importer & Point & & & & Total \\
\hline seller & of & October & November & December & Avg. \\
\hline Purchaser/End User & Entry & Vol. Price & Price & Price & Vol. Price \\
\hline
\end{tabular}

CONTINENTAL ENERGY MARKETING LTD.

Various suppliers

CNE Transmission

Waddington, NY

$44 \quad 2.12$

$0 \quad$ N/A

$0 \quad N / A$

44

2.12

CONTINENTAL ENERGY MARKETING LTD. Wes Cana Energy Marketing Inc. Bethlehem Steel Corporation Niagara Falls, NY $0 \quad$ N/A

$0 \quad$ N/A

31

2.57

31

2.57

CONTINENTAL ENERGY MARKETING LTD.

Wes Cana Energy Marketing Inc.

National Fuel Gas Supply Corporatio Waddington, NY

O N/A

$23 \quad 2.37$

$0 \quad N / A$

23

2.37

CONTINENTAL ENERGY MARKETING LTD.

Wes Cana Energy Marketing Inc.

North East Heat \& Light

Niagara Falls, NY

$0 \quad$ N/A

38

2.57

38

2.57

CONTINENTAL ENERGY MARKETING LTD.

Wes Cana Energy Marketing Inc.

Sunrise Energy

Niagara Falls, NY 0 N/A

$0 \quad$ N/A

48

2.60

48

2.60

CONTINENTAL ENERGY MARKETING LTD. Wes Cana Energy Marketing Inc.

Sunrise Energy

Waddington, NY

O N/A

45

2.32

0

N/A

45

2.32

CRESTAR ENERGY MARKETING CORPORATION

Crestar Energy

Cibola Corporation

Port of Morgan, MT $259 \quad 1.55$

$3 \quad 1.67$

O N/A

262

1.55

CRESTAR ENERGY MARKET:NG CORPORATION

Crestar Energy

N.Y. Z Zate Electric \& Gas

Niagara Falls, NY

O N/A

280

3.21

300

3.20

580

3.20 


$$
\text { Page - } 79
$$

\section{SHORT-TERM SALES}

4th Quarter 1993

Estimated Volumes (MMCF) \& Prices ( $\$ / M M B T U$ ) -- cont.

Quarterly

Total

$\begin{array}{llll}\text { Importer } & \text { Point } & & \\ \text { Seller } & \text { of } & \text { October } & \text { November }\end{array}$

Purchaser/End User

Entry

Vol. Price

Vol. Price

Vol. Price

Vol. Price

CU Energy Marketing, Inc. Developmerit Associates

Sunrise Energy

Eastport, Idaho

O N/A

$59 \quad 1.82$

$0 \quad$ N/A

$59 \quad 1.82$

CU Energy Marketing, Inc. Jonan Gas Marketing Ltd. Sunrise Energy

Eastport, Idaho

O N/A

O N/A

$57 \quad 1.87$

57

1.87

CU Energy Marketing, Inc. PanCanadian Petroleum Ltd.

Sunrise Energy

Eastport, Idaho

O N/A

O N/A

$40 \quad 1.85$

$40 \quad 1.85$

MARTMOUTH POWER ASSOCIATES L.P. Dartmouth Power Associates JMC Fuel Services

Waddington, NY

13

2.15

38

2.37

$0 \quad N / A$

51

2.32

DARTMOUTH POWER ASSOCIATES L.P. Dartmouth Power Associates o \& R Energy Waddington, NY $66 \quad 2.20$

$0 \quad N / A$

15

2.57

81

2.27

DARTMOUTH POWER ASSOCIATES L.P. Dartmouth Power Associates Pawtucket Power Associates Waddington, NY $11 \quad 1.55$

$14 \quad 1.56$

18

1.65

$43 \quad 1.60$

DEKALB ENERGY COMPANY

Dekalb Energy Canada Ltd.

Gaylord Container Corporation

Eastport, Idaho

O N/A

338

1.18

336

1.66

673

1.42

DEKALB ENERGY COMPANY Dekalb Energy Canada Ltd. Redwood Resources Inc. 


\section{Page - 80}

\section{SHORT-TERM SALES \\ 4th Quarter 1993}

Estimated Volumes (MMCF) \& Prices (\$/MMBTU) - - cont.

Importer

Seller

Purchaser/End User
Point

of

Entry
October

Vol. Price
November

Vol. Price
Quarterly

Total

Avg.

December

Vol. Price

DEVELOPMENT ASSOCIATES, INC.

Canadian Natural Resources Limited

Lamb Weston

Sumas, Washington

O N/A

$53 \quad 1.82$

$39 \quad 2.42$

92

2.08

DEVELOPMENT ASSOCIATES, INC.

Canadian Natural Resources Limited

Northwest Alloys

Sumas, Washington

O N/A

$74 \quad 1.82$

$83 \quad 2.43$

158

2.14

DEVELOPMENT ASSOCIATES, INC.

Canadian Natural Resources Limited Potlatch

sumas, Washington $0 \quad \mathrm{~N} / \mathrm{A}$

$40 \quad 1.82$

$72 \quad 2.42$

112

2.21

DEVELOPMENT ASSOCIATES, INC.

Canadian Natural Resources Limited weyerhauser

Sumas, Washington

O N/A

$24 \quad 1.82$

$0 \quad$ N/A

$24 \quad 1.82$

DEVELOPMENT ASSOCIATES, INC.

Wainoco Oil Corporation

potlatch

Sumas, Washington $\quad 114 \quad 1.43$

$54 \quad 1.90$

1.90

$224 \quad 1.66$

OIRECT ENERGY MARKETING LIMITED

Direct Energy Marketing Limited

Cibola Corporation

Port of Morgan, MT $40 \quad 1.61$

O N/A

N/A

$40 \quad 1.61$

DIRECT ENERGY MARKETING LIMITED

Direct Energy Marketing Limited

Facific Gas \& Electric Company

Eastport, Idaho

O N/A

$144 \quad 1.74$

153

2.62

297

2.19

ENRON GAS MARKETING, INC.

Enron Gas Marketing Carada

Northern Illinois Gas

Port of Morgan, MT $1669 \quad 1.58$

1503

1.94

1787

2.06

4960

1.86 
Page - 81

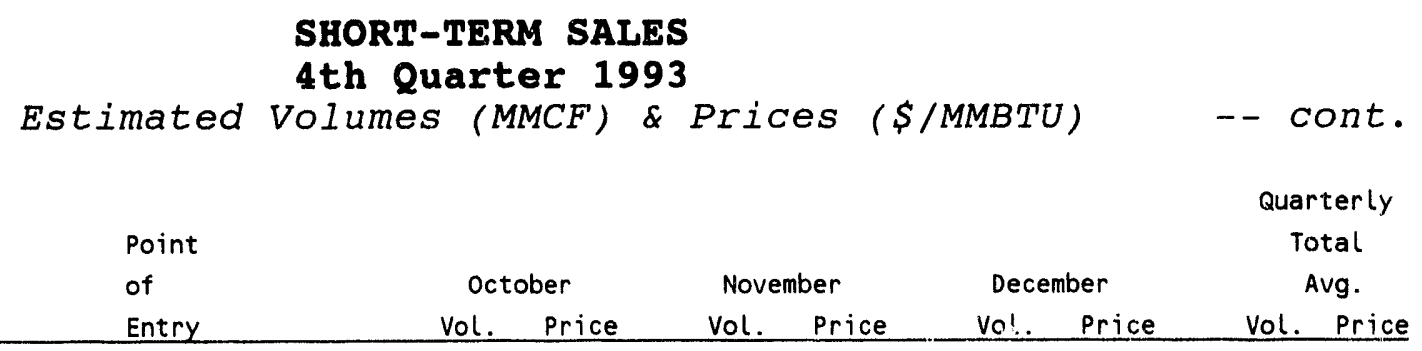

ENRON GAS MARKETING, INC.

Multi-Energies Inc.

Northern Illinois Gas

Port of Morgan, MT $44 \quad 1.36$

$0 \quad N / A$

$0 \quad N / A$

$44 \quad 1.36$

ENRON GAS MARKETING, INC.

North Canadian Marketing Inc.

Northern Illinois Gas

Port of Morgan, MT $160 \quad 1.11 \quad 307 \quad 1.78$

467

1.55

ENRON GAS MARKETING, INC.

Unigas Corporation

Northern Illinois Gas

Port of Morgan, MT 651

$0 \quad N / A$

$651 \quad 1.10$

GOETZ OIL CORPORATION

KCS Energy Management Services

Blaw Knox

Niagara Falls, NY $\quad 1 \quad 2.20$

$0 \quad N / A$

O N/A

$1 \quad 2.20$

GOETZ OIL CORPORATION

KCS Energy Management Services

Carborundum Abrasives

Niagara Falls, MY $\quad 14 \quad 2.20 \quad 0 \quad$ N/A

D N/A

$14 \quad 2.20$

GOETZ OIL CORPORATION

KCS Energy Management Services

FMC

Niagara Falls, NY 12.20

$0 \quad N / A$

O N/A

2.20

GOETZ OIL CORPORATION

KCS Energy Management Services

Goetz Energy Corporation

Niagar a Falls, NY 12.20

.20

$0 \quad \mathrm{~N} / \mathrm{A}$

$0 \quad$ N/A

2.20

GRAND VALLEY GAS COMPANY

Grand Valley Gas Canada Ltd.

Aluminum Company of America

Sumas, Washington $\quad 36 \quad 1.70$

$0 \quad N / A$

D N/A

36

1.70 


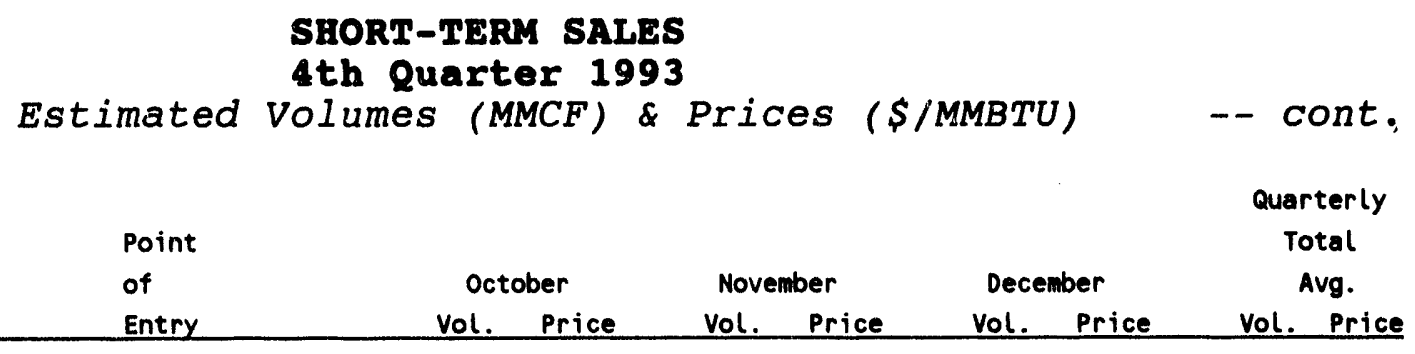

GRAND VALLEY GAS COMPANY Grand Valley Gas Canada Ltd. American Hunter Energy Sumas, Washington $237 \quad 1.70$ $55 \quad 2.44$

D N/A 292 1.84

GRAND VALLEY GAS COMPANY Grand Valley Gas Canada Ltd. BC Gas Inc.

GRAND VALLEY GAS COMPANY Grand Valley Gas Canada Ltd. Biollass

GRAND VALLEY GAS COMPANY Grand Valley Gas Canada Ltd. Boeing Company

GRAND VALLEY GAS COMPANY Grand Valley Gas Canada Ltd. Cascade Natural Gas

$\begin{array}{lllllllll}\text { Sumas, Washington } & 305 & 1.86 & 85 & 2.23 & 35 & 2.76 & 425 & 2.01\end{array}$

GRAND VALLEY GAS COMPANY Grand Valley Gas Canada Ltd. Cascade Steel 


$$
\text { Page - } 83
$$

SHORT-TERM SALES
4th Quarter 1993
Estimated Volumes (MMCF) \& Prices (S/MMBTU)

GRAND VALLEY GAS COMPANY Grand Valley Gas Canada Ltd.

Coastal Gas Marketing Company

Sumas, Washington $\quad 0 \quad N / A$

O N/A

$47 \quad 2.50$

47

2.50

GRAND VALLEY GAS COMPANY Grand Valley Gas Canada Ltd. Derigold Inc.

Sumas, Washington

O N/A

$20 \quad 2.20$

$0 \quad$ N/A

20

2.20

GRAND VALLEY GAS COMPANY Grand Valley Gas canada Ltd. Destec

GRAND VALLEY GAS COMPANY Grand Valley Gas Canada Ltd. Development Associates

GRAND VALLEY GAS COMPANY Grand Valley Gas canada Ltd. Domtar Gypsum

GRAND VALLEY GAS COMPANY Grand Valley Gas Canada Ltd. Elf Atochem 


\section{SHORT-TERM SALES \\ 4th Quarter 1993}

Estimated Volumes (MMCF) \& Prices (\$/MMBTU) -- cont.

\begin{tabular}{|c|c|c|c|c|c|c|}
\hline \multirow{3}{*}{ Importer } & \multirow[b]{2}{*}{ Point } & & & & & \multirow{2}{*}{$\begin{array}{c}\text { Quarterly } \\
\text { Total }\end{array}$} \\
\hline & & & & & & \\
\hline & of & october & November & & ber & Avg. \\
\hline Purchaser/End User & Entry & Price & Price & Vol & Price & Price \\
\hline
\end{tabular}

GRAND VALLEY GAS COMPANY Grand Valley Gas Canada Ltd. Evanite Sumas, Washington $34 \quad 1.70$

16

2.22

0

N/A

50

1.87

GRAND VALLEY GAS COMPANY Grand Valley Gas Canada Ltd. General Chemical

GRAND VALLEY GAS COMPANY Grand Valley Gas Canada Ltd. Georgia Pacific Corporation

GRAND VALLEY GAS COMPANY Grand Valley Gas Canada Ltd. Grand Valley Gas Company

Eastport, Idaho $26 \quad 1.76$

$0 \quad N / A$

$228 \quad 2.44$

258

2.52

1059

2.08

Grand Vailey Gas Company

GRAND VALLEY GAS COMPANY

Grand Valley Gas Canada Ltd.

IGI Resources

GRAND VALLEY GAS CUMPANY

Grand Valley Gas Canada Ltd.

IGI Resources

Eastport, Ldaho

$0 \quad N / A$

23

Sumas, Washington

903

1.81

$340 \quad 2.30$

354

2.75

1597

2.12

GRAND VALLEY GAS COMPANY Grand Valley Gas Canada Ltd. J. Aaron Company
Sumas, Washington

O N/A

350

2.20 


$$
\text { Page - } 85
$$

\section{SHORT-TERM SALES \\ 4th Quarter 1993 \\ Estimated Volumes (MMCF) \& Prices (\$/MMBTU) -- cont.}

\begin{tabular}{|c|c|c|c|c|c|}
\hline Importer & Point & & & & $\begin{array}{c}\text { Quarterly } \\
\text { Total }\end{array}$ \\
\hline Selier & of & October & November & December & Avg. \\
\hline Purchaser/End User & Entry & Vol. Price & Vol. Price & Price & Vol. Price \\
\hline
\end{tabular}

GRAND VALLEY GAS COMPANY

Grand Valley Gas Canada Ltd.

James River Corporation

GRAND VALLEY GAS COMPANY

Grand Valley Gas Canada Ltd.

National Gas Resources

GRAND VALLEY GAS COMPANY

Grand Valley Gas Canada Ltd.

Northwest Aluminum Company

GRAND VALLEY GAS COMPANY

Grand Valley Gas Canada Ltd.

Pacific Gas \& Electric Company

GRAND VALLEY GAS COMPANY

Grand Valley Gas Canada Ltd.

Pacific Gas \& Electric Company

GRAND VALLEY GAS COMPANY

Grand Valley Gas Canada Ltd.

Petro Canada Hydrocarbons

GRAND VALLEY GAS COMPANY

Grand Valley Gas Canada Ltd.

Portland General Electric Company

Eastport, Idaho $\quad 148 \quad 1.87$

$0 \quad N / A$

N/A

148

1.87

GRAND VALLEY GAS COMPANY

Grand Valley Gas Canada Ltd.

Portland General Electric Company

Sumas, Washington

428

1.70

73

2.34

0

N/A

502

1.79 


$$
\text { Page - } 86
$$

\section{SHORT-TERM SALES \\ 4th Quarter 1993}

Estimated Volumes (MMCF) \& Prices (\$/MMBTU) -- cont.

Importer

seller

Purchaser/End User

GRAND VALLEY GAS COMPANY

Grand Valley Gas Canada Ltd.

Premier Edible oils

GRAND VALLEY GAS COMPANY Grand Valley Gas Canada Ltd. Questar Pipeline Co.

GRAND VALLEY GAS COMPANY Grand Valley Gas Canada Ltd. Scott Paper

GRAND VALLEY GAS COMPANY Grand Valley Gas Canada l.td. seattle Steam

GRAND VALLEY GAS COMPANY Grand Valley Gas Canada Ltd. Sierra Pacific Power Company

GRAND VALLEY GAS COMPANY Grand Valley Gas Canada Ltd. Simpson Tacoma Kraft Company

GRAND VALLEY GAS COMPANY Grand Valley Gas Canada Ltd. Teledyne

GRAND VALLEY GAS COMPANY Grand Valley Gas Canada Ltd. Unocal
Sumas, Washington

$17 \quad 1.70$

18

2.20

18

2.78

53

2.23

Sumas, Washington

$77 \quad 1.99$

O N/A

$0 \quad$ N/A

$77 \quad 1.99$

Sumas, Washington $\quad 121 \quad 1.70$

O N/A

0

N/A

121

1.70
$96 \quad 1.70$

130

2.35

90

2.52

316

2.20

Sumas, Washington $\quad 197 \quad 1.70$

$6 \quad 2.20$

24

2.78

227

1.83
$0 \quad$ N/A

O N/A

143

1.70
Sumas, Washington $25 \quad 1.70$

25

2.20

19

2.36

70

2.06
Sumas, Washington $\quad 8 \quad 1.70$
$4 \quad 2.23$
12

2.36
24

2.12 


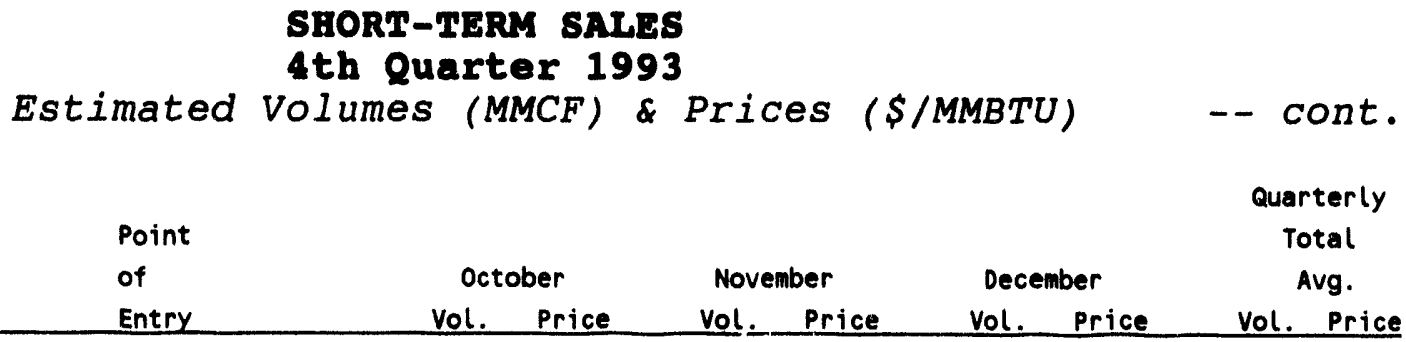

GRAND VALLEY GAS COMPANY Grand Valley Gas Canada Ltd. Utah Gas Services

$1 \quad 2.78$

8

2.43

GRAND VALLEY GAS COMPANY

Grand Valley Gas Canada Ltd.

Washington Water Power Company

Eastport, Idaho

$220 \quad 1.76$

95

2.00

1345

2.14

1660

2.08

GRAND VALLEY GAS COMPANY

Grand Valley Gas Canada Ltd.

Washington Water Power Company

Sumas, Washington

$0 \quad$ N/A

$0 \quad$ N/A

185

2.75

185

2.75

GRAND VALLEY GAS COMPANY Grand Valley Gas Canada Ltd.

Weyerhauser

Eastport, Idaho

$0 \quad N / A$

89

2.00

110

2.14

199

2.08

GRAND VALLEY GAS COMPANY

Grand Valley Gas Canada Ltd.

Weyerhauser

Sumas, Washington

138

1.94

77

2.20

241

2.63

457

GRAND VALLEY GAS COMPANY Grand Valley Gas Canada Ltd. Wyoming Industrial Gas

GRANITE STATE GAS TRANSMISSION, INC.

Direct Energy Marketing Limited Granite State Gas Transmission, Inc North Troy, VT

GREAT FALLS GAS COMPB... $\mathbf{S P}$

Bearpaw Gathering Systans, Inc.

Great Falls Gas Company

Babb, Montana

$0 \quad$ N/A

$45 \quad 2.02$

186

2.52

231

2.42

SP - Interruptible/special purchase sale made under long-term authorization 


\section{SHORT-TERM SALES}

4th Quarter 1993

Estimated Volumes (MMCF) \& Prices (\$/MMBTU) - - cont.

Quarterly

Total

Importer Point

of October November December Avg.

Seller
Purchaser/End User

Entry

Vol. Price

Vol. Price

Vol.

Price

Vol. Price

GREAT FALLS GAS COMPANY SP

Interenergy Corporation

Great Falls Gas Company

Babb, Montana

$0 \quad N / A$

68

1.99

140

2.52

208

2.35

GREAT PLAINS NATURAL GAS COMPANY

West coast Gas Services Inc.

Great Plains Natural Gas Company

Port of Morgan, MT $0 \quad$ N/A

$10 \quad 1.97$

$10 \quad 2.49$

20

2.23

GREAT PLAINS NATURAL GAS COMPANY

Western Gas Marketing Limited

Great Plains Natural Gas Company

Noyes, Minnesota

$0 \quad N / A$

$8 \quad 2.14$

$8 \quad 2.44$

15

2.29

GREAT WEST ENERGY LTD.

Great West Energy Ltd.

Acco World-East

Massena, New York 11.95

$1 \quad 1.95$

$1 \quad 1.95$

2

1.95

GREAT WEST ENERGY LTD. Great West Energy Ltd.

Acco World-West

Massena, New York 11.95

$1 \quad 1.95$

$1 \quad 1.95$

3

1.95

GREAT WEST ENERGY LTD.

Great West Energy Ltd.

Canton Central School

Massena, New York

2

1.95

$1 \quad 1.95$

$2 \quad 1.95$

$5 \quad 1.95$

GREAT WEST ENERGY LTD.

Great West Energy Ltd.

Canton-Potsdam Hospital

Massena, New York

$2 \quad 1.95$

$1 \quad 1.95$

$2 \quad 1.95$

$5 \quad 1.95$

GREAT WEST ENERGY LTD.

Great West Energy Ltd.

City Ogdensburg, NY

Massena, New York

31.95

31.95

31.95

10

1.95

SP - Interruptible/special purchase sale made under long-term authorization 
Page - 89

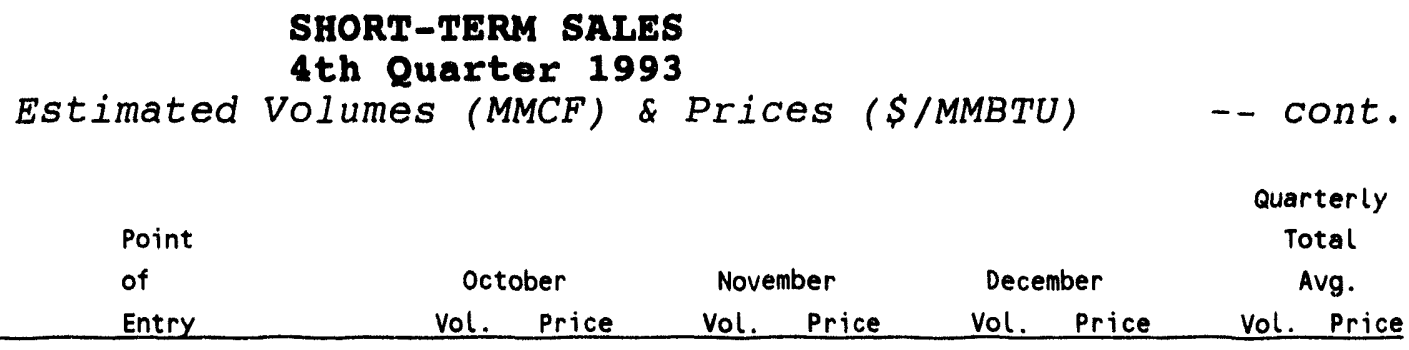

Importer

Seller

Entry

Vol.

Price

vol. Price

Vol. Price

Vol. Price

GREAT WEST ENERGY LTD.

Great West Energy Ltd.

Cowest Energy

St. Clair, MI

D N/A

$0 \quad N / A$

500

2.82

500

2.82

GREAT WEST ENERGY LTD. Great West Energy Ltd.

$F$ landers Inn

Massena, New York

$0 \quad 1.95$

$0 \quad 1.95$

$0 \quad 1.95$

1

1.95

GREAT WEST ENERGY LTD. Great West Energy Ltd. Highland Nursing Home

GREAT WEST ENERGY LTD. Great West Energy Ltd. Hoosier Magnetics, Inc.

GREAT WEST ENERGY LTD. Great West Energy Ltd. Joanna A. CHF Company

GREAT WEST ENERGY LTD. Great West Energy Ltd. Potsdam Central school

N/A

GREAT WEST ENERGY LTD. Great West Energy Ltd. St. Regis Nursing Home 


$$
\text { Page - } 90
$$

\section{SHORT-TERM SALES \\ 4th Quarter 1993}

Estimated Volumes (MMCF) \& Prices (\$/MMBTU) -- cont.

Quarterly

Total

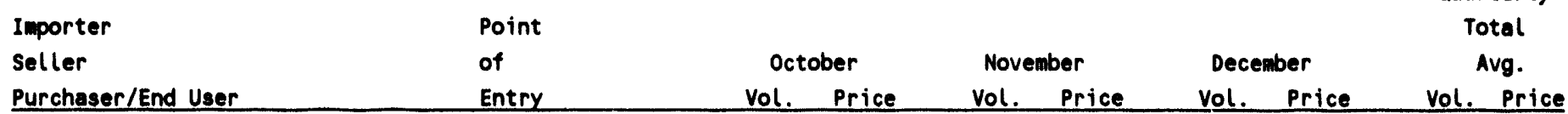

GREAT WEST ENERGY LTD.

Great West Energy Ltd.

Stella Cheese Company

Massena, New York

$5 \quad 1.95$

$5 \quad 1.95$

$5 \quad 1.95$

$16 \quad 1.95$

GREAT WEST ENERGY LTD.

Great West Energy Ltd.

United Helpers Management Company

Massena, New York

$1 \quad 1.95$

$1 \quad 1.95$

$1 \quad 1.95$

$4 \quad 1.95$

GREAT WEST ENERGY LTD.

Great West Energy Ltd.

Village of Potsdan Housing Authorit

Massena, New York

$1 \quad 1.95$

$1 \quad 1.95$

$1 \quad 1.95$

31.95

HOWARD ENERGY COMPANY, INC.

Northridge Petroleum Marketing, Inc.

Howard Energy Company

St. Clair, MI

210

2.17

$0 \quad$ N/A

$0 \quad$ N/A

210

2.17

HUSKY GAS MARKETING, INC.

Husky Oil

Aquila Energy Marketing

Port of Morgan, MT $302 \quad 1.20$

$63 \quad 1.58$

$0 \quad$ N/A

366

1.27

HUSKKY GAS MARKETING, INC.

Husky Oil

Bridgegas

Sumas, Washington $121 \quad 1.34$

$\begin{array}{ll}58 & 1.79\end{array}$

O N/A

179

1.48

HUSKY GAS MARKETING, INC.

Husky Oil

cibola corporation

Port of Morgan, MT

639

1.30

$544 \quad 1.58$

654

1.65

1837

1.51

HUSKY GAS MARKETING, INC.

Husky Oil

Coastal Gas Marketing Company

Port of Morgan, MT

$0 \quad N / A$

$406 \quad 1.78$

302

2.27

708

1.99 


$$
\text { Page - } 91
$$

ATTACHMENT $\mathrm{C}-4$

SHORT-TERM SALES
4th Quarter 1993
Estimated Volumes (MMCF) \& Prices (S/MMBTU)

Seller

Entry

Vol. Price

Vol. Price

Vol.

Price

Vol. Price

HUSKY GAS MARKETING, INC.

Husky Oil

Coastal Gas Marketing Company

Sumas, Washington $0 \quad$ N/A

$7 \quad 2.90$

42

2.02

50

2.15

HUSKY GAS MARKETING, INC.

Husky Oil

IGI Resources

Sumas, Washington $0 \quad N / A$

$77 \quad 1.78$

$256 \quad 2.00$

332

1.95

HUSKY GAS MARKETING, INC.

Husky oil

Louis Dreyfus Energy Corporation

Port of Morgan, MT $605 \quad 1.08$

$0 \quad$ N/A

$0 \quad$ N/A

$605 \quad 1.08$

HUSKY GAS MARKETING, INC.

Husky Oil

sioux Pointe Inc.

Port of Morgan, MT $605 \quad 1.36$

$0 \quad N / A$

$220 \quad 1.84$

824

1.48

HUSKY GAS MARKETING, INC.

Husky Oil

Tenaska Marketing Ventures

Port of Morgan, MT $455 \quad 1.22$

$833 \quad 1.72$

967

1.55

2255

1.55

HUSKY GAS MARKETING, INC.

Husky Oil

Terra Industries

Port of Morgan, MT 454

Sumas, Washington $120 \quad 1.57$

$152 \quad 1.85$

O N/A

272

1.73

HUSKY GAS MARKETING, INC.

Husky Oil

Washington Natural Gas Company

Sumas, Washington $\quad 0 \quad N / A$

270

2.43

164

3.50

434

2.83 


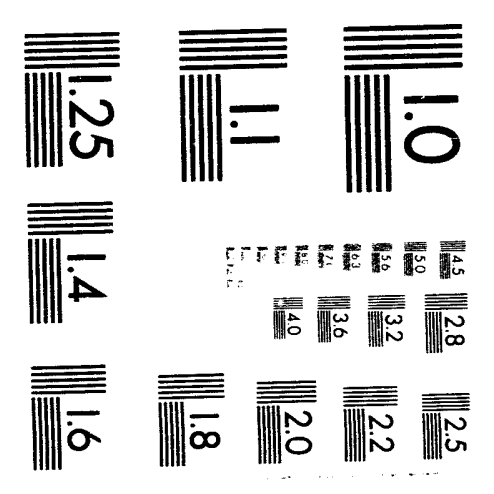




$$
\stackrel{N}{\stackrel{N}{N}}
$$




$$
\text { Page - } 92
$$

\section{SHORT-TERM SALES \\ 4th Quarter 1993}

Estimated Volumes (MMCF) \& Prices (\$/MMBTU) -- cont.

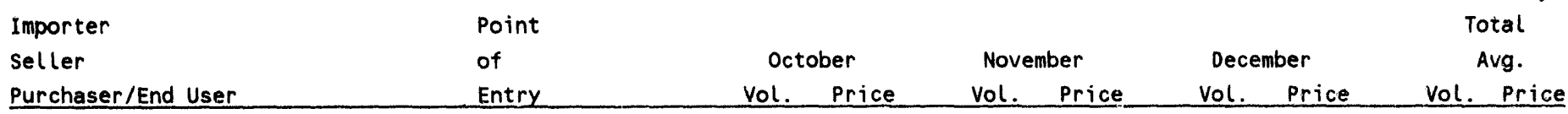

IGI RESOURCES INC.

Amoco Canada Marketing Corporation

Cascade Natural Gas

Eastport, Idaho

O N/A

116

2.01

$\begin{array}{ll}54 & 2.09\end{array}$

170

2.04

IGI RESOURCES INC.

CanWest Gas Supply Inc.

J.R. Simplot Company

Sumas, washington $153 \quad 1.60$

$0 \quad N / A$

$0 \quad$ N/A

$153 \quad 1.60$

IGI RESOURCES INC.

CanWest Gas Supply Inc.

Southwest Gas Corporation

Sumas, Washington

O N/A

$158 \quad 1.80$

D N/A

$158 \quad 1.80$

IGI RESOURCES INC.

Kimball Energy Corporation

WP Natural Gas

Eastport, Idaho

O N/A

$111 \quad 2.04$

O N/A

111

2.04

IGI RESOURCES INC.

Mobil Oil Canada Lta.

Amalgamated Sugar Company

Sumas, Washington

$3 \quad 1.39$

$4 \quad 2.59$

$4 \quad 2.03$

$11 \quad 2.07$

IGI RESOURCES INC.

Mobil Oil Canada Ltd.

American fine Foods

Sumas, Washington

$7 \quad 1.39$

$0 \quad 2.59$

12.03

$8 \quad 1.48$

IGI RESOURCES INC.

Mobil Oil Canada Ltd.

Armstrong World Industries, Inc.

Sumas, Washington

$51 \quad 1.39$

$7 \quad 2.59$

$51 \quad 2.03$

109

1.77

IGI RESOURCES INC.

Mobil Oil Canada Ltd.

Ash Grove Cement

Sumas, Washington $\quad 36 \quad 1.39$

$39 \quad 2.59$

$0 \quad$ N/A

$75 \quad 2.02$ 


$$
\text { Page - } 93
$$

SHORT-TERM SALES
4th Quarter 1993
Estimated Volumes (MMCF) \& Prices (S/MMBTU)

IGI RESOURCES INC.

Mobil Oil Canada Ltd.

Ash Grove Cement West

Sumas, Washington $\quad 65 \quad 1.39$

23

2.59

12.03

$89 \quad 1.71$

IGI RESOURCES INC.

Mobil oil Canada Ltd.

Ault Naval Base

Sumas, Washington $\quad 16 \quad 1.39$

$15 \quad 2.59$

$9 \quad 2.03$

40

1.99

IGI RESOURCES INC.

Mobil Oil Canada Ltd.

Basic American Foods

Sumas, Washington $\quad 36 \quad 1.39$

$0 \quad 2.59$

$6 \quad 2.03$

42

1.49

IGI RESOURCES INC.

Mobil Oil Canada Ltd.

Boise Cascade Corporation

Sumas, Washington

$83 \quad 1.39$

$147 \quad 2.59$

284

2.03

515

2.09

IGI RESOURCES INC.

Mobil Oil Canada Ltd.

Carnation Dairies

Sumas, Washington

$50 \quad 1.39$

$46 \quad 2.59$

69

2.03

165

1.99

IGI RESOURCES INC.

Mobil Oil Canada Ltd.

ESCO Corporation

Sumas, Washington

$11 \quad 1.39$

$0 \quad$ N/A

$8 \quad 2.03$

$19 \quad 1.66$

¿II RESOURCES INC.

Mobil Oil Canada Ltd.

Endurance Fruit

Sumas, Washington

$14 \quad 1.39$

14

2.59

10

2.03

$38 \quad 2.00$

IGI RESOURCES INC. Mobil Oil Canada Ltd.

Grand Valley Gas Company

O N/A

O N/A

209

1.39 


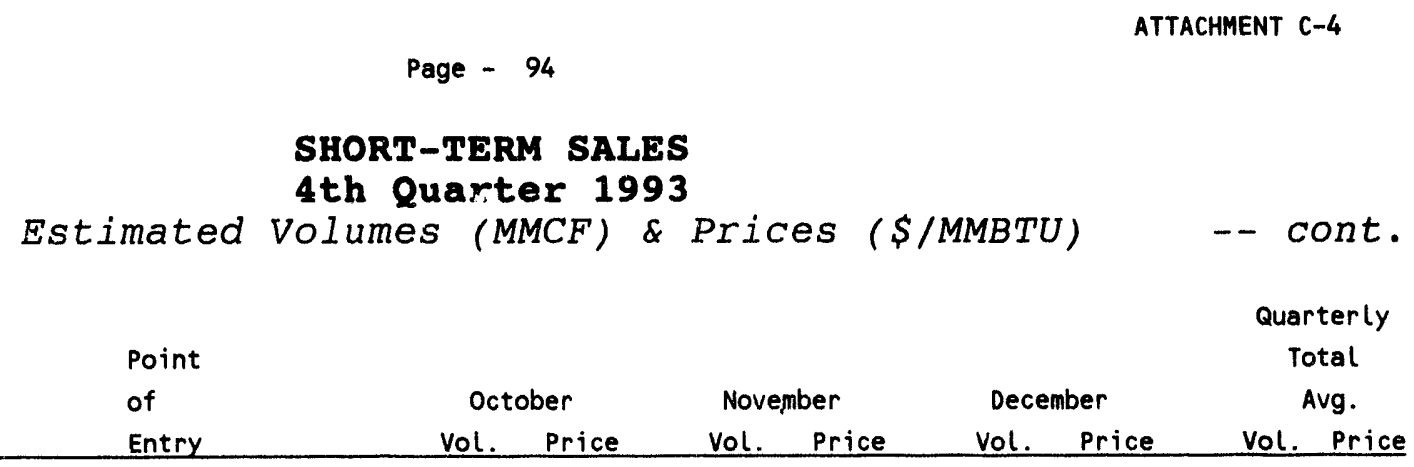

IGI RESOURCES INC.

Mobil Oil Canada Ltd.

IBP

Sumas, Washington

$27 \quad 1.39$

25

2.59

26

2.03

$\begin{array}{ll}78 & 1.99\end{array}$

IGI RESOURCES INC. Mobil Oil Canada Ltd.

IGI Resources

Sumas, Washington

$0 \quad N / A$

$14 \quad 2.59$

$0 \quad$ N/A

14

2.59

IGI RESOURCES INC.

Mobil Oil Canada Ltd.

Interox

Sumas, Washington

$47 \quad 1.39$

$52 \quad 2.59$

55

2.03

154

2.02

IGI RESOURCES INC.

Mobil Oil Canada Ltd.

J.R. Simplot Company

Sumas, Washington

$183 \quad 1.39$

213

2.59

53

2.03

449

2.03

IGI RESOURCES INC.

Mobil Oil Canada Ltd.

KTM

Sumas, Washingto

18

1.39

189

2.59

$81 \quad 2.03$

$458 \quad 2.00$

IGI RESOURCES INC.

Mobil Oil Canada Ltd.

Legacy Health System

Sumas, Washington

$21 \quad 1.39$

O N/A

$21 \quad 2.03$

42

1.72

IGI RESOURCES INC. Mobil Oil Canada Ltd. Naval Air Station

IGI RESOURCES INC. Mobil Oil Canada Ltd. Oroweat 


$$
\text { Page - } 95
$$

\section{SHORT-TERM SALES \\ 4th Quarter 1993}

Estimated Volumes (MMCF) \& Prices (\$/MMBTU) -- cont.

$\begin{array}{llll}\text { Importer } & \text { Point } & & \\ \text { Seller } & \text { of } & \text { Octoberterly } & \text { Total }\end{array}$

Purchaser/End User

Entry

vol. Price

Vol. Price

Vol. Price

Vol. Price

IGI RESOURCES INC.

Mobil Oil Canada Ltd.

PACCO

Sumas, Washington

$29 \quad 1.39$

1

2.59

31

2.03

$61 \quad 1.73$

IGI RESOURCES INC.

Mobil Oil Canada Ltd.

Pacific Gas Electric Company

Sumas, Washington

$71 \quad 1.39$

O N/A

O N/A

$71 \quad 1.39$

IGI RESOURCES INC.

Mobil Oil Canada Ltd.

Roseburg Lumber

Sumas, Washington

$12 \quad 1.39$

$4 \quad 2.59$

19

2.03

$35 \quad 1.88$

IGI RESOURCES INC.

Mobil Oil Canada Ltd.

Sierra Pacific Power Company

Sumas, Washington

$5 \quad 1.39$

$0 \quad$ N/A

$0 \quad N / A$

$5 \quad 1.39$

IGI RESOURCES INC.

Mobil Oil Canada Ltd.

Southwest Gas Corporation

Sumas, Washington

O N/A

$25 \quad 2.59$

O N/A

$25 \quad 2.59$

IGI RESOURCES INC.

Mobil Oil Canada Ltd.

Universal frozen foods

Sumas, Washington $\quad 69 \quad 1.39$

$\begin{array}{ll}66 & 2.59\end{array}$

$67 \quad 2.03$

$201 \quad 2.00$

IGI RESOURCES INC.

Mobil Oil Canada Ltd.

va Medical

Sumas, Washington

$0 \quad 1.39$

$0 \quad N / A$

$21 \quad 2.03$

$22 \quad 2.02$

IGI RESOURCES INC.

Mobil Oil Canada Ltd.

WP Natural Gas

Sumas, Washington $291 \quad 1.39$

$35 \quad 2.59$

$192 \quad 2.03$

$518 \quad 1.71$ 


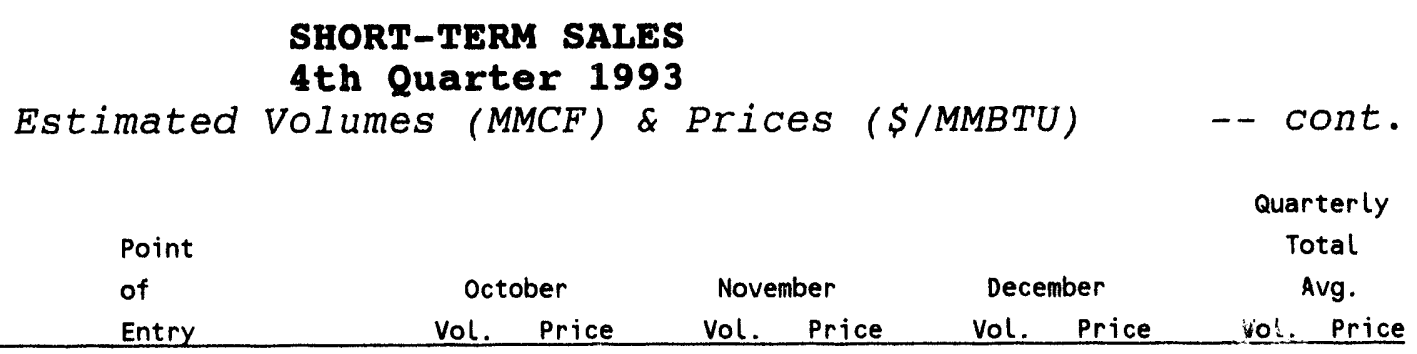

IGI RESOURCES INC.

Mobil Oil Canada Ltd.

Willamette

Sumas, Washington

$5 \quad 1.39$

$0 \quad N / A$

O N/A

$5 \quad 1.39$

IGI RESOURCES INC.

Talisman

Ash Grove Cement West

Eastport, Idaho

o N/A

$12 \quad 1.88$

$0 \quad$ N/A

12

1.88

IGI RESOURCES INC.

Talisman

Basic American Foods

Eastport, Idaho

$0 \quad N / A$

$1 \quad 1.88$

$0 \quad N / A$

$1 \quad 1.88$

IGI RESOURCES INC.

Talisman

Boise Cascade Corporation

Eastport, Idaho

$0 \quad N / A$

$5 \quad 1.88$

$6 \quad 2.35$

11

2.13

IGI RESOURCES INC.

Talisman

ESCO Corporation

Eastport, Idaho

$0 \quad$ N/A

$0 \quad N / A$

1

2.35

1

2.35

IGI RESOURCES INC.

Talisman

J.R. Simplot Company

Eastport, Idaho

$0 \quad N / A$

$239 \quad 1.88$

338

2.35

577

2.16

IGI RESOURCES INC.

Talisman

PACCO

Eastport, Idaho

$0 \quad N / A$

O N/A

33

2.35

$33 \quad 2.35$

IGI RESOURCES INC.

Talisman

Roseburg Lumber

Eastport, Idaho

$0 \quad$ N/A

$0 \quad N / A$

1

2.35

1

2.35 


\section{SHORT-TERM SALES}

4th Quarter 1993

Estimated Volumes (MMCF) \& Prices (\$/MMBTU) -- cont.

\begin{tabular}{|c|c|c|c|c|c|c|c|c|}
\hline Importer & Point & & & & & & & Total \\
\hline Seller & of & & ber & Nov & per & & ber & Avg. \\
\hline Purchaser/End User & Entry & Vol. & Price & Vol. & Price & Vol & Price & Price \\
\hline
\end{tabular}

IGI RESOURCES INC.

Talisman

Sierra Pacific Power Company

Eastport, Idaho

O N/A

$10 \quad 1.88$

o N/A

10

1.88

IGI RESOURCES INC.

Talisman

VA Medical

Eastport, Idaho

O N/A

O N/A

$0 \quad 2.35$

$0 \quad 2.35$

IGI RESOURCES INC.

Talisman

WP Natural Gas

Eastport, Idaho

$0 \quad N / A$

$0 \quad N / A$

12

2.35

12

2.35

IGI RESOURCES INC.

Talisman

WP Natural Gas

Sumas, Washington

O N/A

$14 \quad 1.88$

$0 \quad N / A$

$14 \quad 1.88$

IGI RESOURCES INC.

Unigas Corporation

Cascade Natural Gas

INDECK ENERGY SERVICES, INC.

Continental Energy Marketing Ltd.

Indeck Energy Services of Oswego, I Niagara Falls, NY

D N/A

$75 \quad 2.16$

$\begin{array}{ll}76 & 2.31\end{array}$

151

2.24

INDECK ENERGY SERVICES, INC.

Northstar Energy Corporation

Indeck Energy Services of Yerkes, I Niagara Falls, NY

$40 \quad 1.02$

$42 \quad 1.53$

44

1.54

125

1.37

INDECK ENERGY SERVICES, INC.

Rigel Energy

Indeck Energy Services of Yerkes, I Niagara Falls, NY

O N/A

$0 \quad$ N/A

$5 \quad 2.39$

5

2.39 


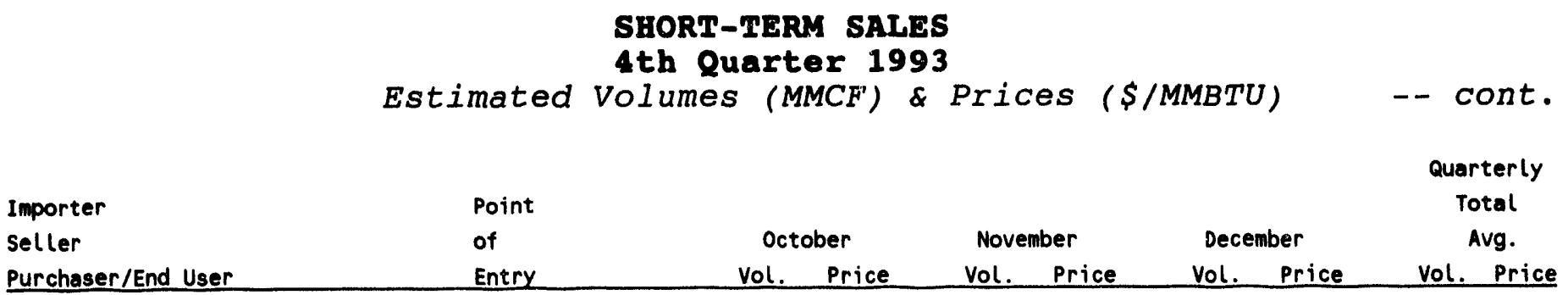

INDECK ENERGY SERVICES, INC.

Transcanada Pipelines Limited

Indeck Energy Services of Yerkes, I Niagara Falls, NY $\quad \begin{array}{lllllll}0 & \text { N/A } & 0 & \text { N/A } & 13 & 1.51 & 13\end{array}$

INDECK ENERGY SERVICES, INC.

Wes Cana Energy Marketing Inc.

Indeck Energy Services of Oswego, I Niagara Falls, NY

$72 \quad 1.51$

$0 \quad N / A$

$0 \quad N / A$

$72 \quad 1.51$

INLAND NATURAL GAS MARKETING ITD.

Inland Natural Gas

Canadian Hydrocarbons

Sumas, Washington

O N/A

$0 \quad N / A$

17

2.15

17

2.15

INLAND NATURAL GAS MARKETING LTD.

Inland Natural Gas

Cascade Natural Gas

Sumas, Washington $\quad 0 \quad N / A$

$90 \quad 1.91$

103

2.18

193

2.05

INLAND MATURAL GAS MARKETING LTD.

Inland Natural Gas

Development Associates

Sumas, Washington

$0 \quad N / A$

O N/A

76

2.10

76

2.10

INLAND NATURAL GAS MARKETING LTD.

Inland Natural Gas

J. Aaron Company

Sumas, Washington

$0 \quad$ N/A

$20 \quad 1.85$

O N/A

$20 \quad 1.85$

INLAND MATURAL GAS MARKETING LTD.

Inland Natural Gas

Natural Gas Clearinghouse

Sumas, Washington $\quad 0$ N/A

28

2.55

O N/A

28

2.55

INLAND MATURAL GAS MARKETING LTO.

Inland Natural Gas

Petro Canada Hydrocarbons 


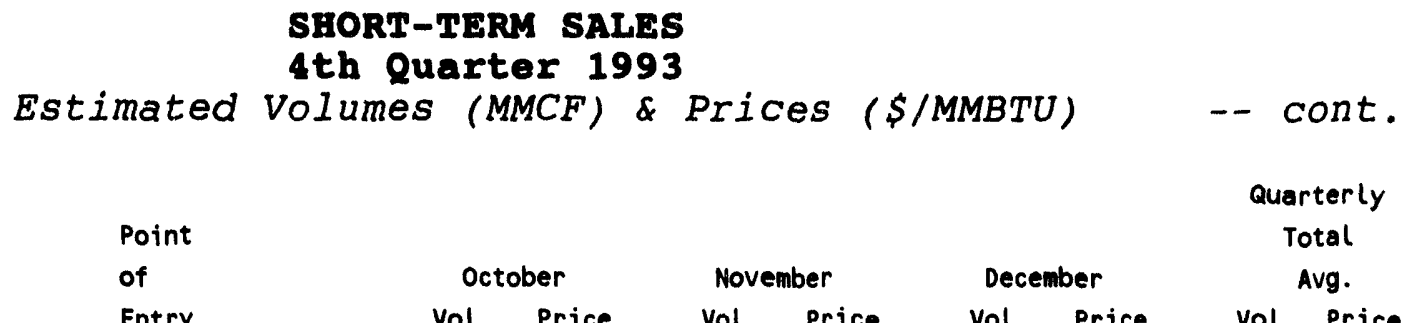

Importer

Entry

Vol.

Vol.

Vol.

Vol. Price

INTALCO ALUMINUM CORPORATION

CanHest Gas Supply Inc.

Intalco Aluminum Corporation

Sumas, Washington

$39 \quad 1.40$

$83 \quad 1.96$

89

2.62

212

2.13

INTERENERGY CORPORATION

Northridge Petroleum Marketing, Inc.

Interenergy Corporation

Babb, Montana

$40 \quad 1.34$

O N/A

$0 \quad$ N/A

$40 \quad 1.34$

INTERENERGY CORPORATION

Various Suppliers

Interenergy Corporation

Babb, Montana

$0 \quad$ N/A

o N/A

29

1.80

$29 \quad 1.80$

INTERENERGY CORPORATION

Western Gas Marketing Limited

Interenergy Corporation

Babb, Montana

O N/A

$9 \quad 1.47$

$0 \quad N / A$

$9 \quad 1.47$

INTERSTATE POWER COMPANY

Unigas Corporation

Interstate Power Company

Port of Morgan, MT

$0 \quad N / A$

20

2.11

20

2.64

$40 \quad 2.37$

IOWA ELECTRIC LIGHT \& POWER COMPANY Unigas Corporation

lowa Electric Light Power

Port of Morgan, MT $0 \quad$ N/A

$82 \quad 1.98$

85

2.49

168

2.24

IROQUOIS ENERGY MANAGEMENT, INC. Direct Energy Marketing Limited SUNY - N.Y.

Niegara Falls, NY

$0 \quad N / A$

O N/A

233

2.50

233

2.50

IROAUOIS ENERGY MANAGENENT, INC. Enron Gas Marketing Canada SUNY - N.Y. 


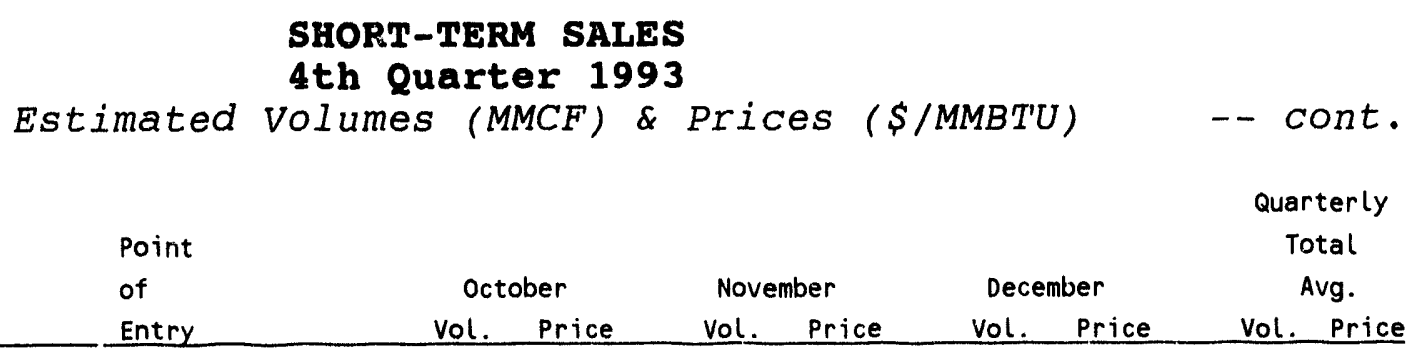

Importer

Entry

Vol. Price

Vol. Price

Vol.

Price

Vol. Price

IROQUOIS ENERGY MANAGEMENT, INC.

KCS Energy Management Services

SUNY - N.Y.

Niagara Falls, NY

$0 \quad N / A$

109

2.30

$0 \quad$ N/A

109

2.30

JONAN GAS MARKETING

Jonan Gas Marketing Ltd.

Sunrise Energy

Eastport, Idaho

$0 \quad N / A$

$21 \quad 1.76$

$28 \quad 1.96$

$49 \quad 1.88$

KCS ENERGY MARKETING, INC.

Bow Valley Energy Inc.

Kamine/Besicorp Natural Dam L.P. Waddington, NY

$0 \quad N / A$

$17 \quad 2.65$

$7 \quad 3.32$

24

2.84

KCS ENERGY MARKETING, INC.

Renaissance Energy Ltd.

Kamine/Besicorp Carthage L.P.

Grand Is land, NY 0 N/A

$0 \quad N / A$

21

2.93

21

2.93

KCS ENERGY MARKETING, INC.

Renaissance Energy Ltd.

Kamine/Besicorp Carthage L.P

Waddington, NY

63

2.19

$0 \quad N / A$

$0 \quad N / A$

63

2.19

KCS ENERGY MARKETING, INC.

Renaissance Energy Ltd.

Kamine/Besicorp South Glens Falls

Noyes, Minnesota $106 \quad 1.69$

$40 \quad 1.70$

41

2.24

187

1.81

KIMBALL ENERGY CORPORATION Canadian Hydrocarbons Marketing Canadian Hydrocarbons

KIMBALL ENERGY CORPORATION

Canadian Hydrocarbons Marketing

Coastal Gas Marketing Company

Eastport, Idaho $\quad 181 \quad 1.60$

$0 \quad N / A$

$0 \quad$ N/A

181

1.60 


$$
\text { Page - } 101
$$

\section{SHORT-TERM SALES \\ 4th Quarter 1993 \\ Estimated Volumes (MMCF) \& Prices (\$/MMBTU) - cont.}

\begin{tabular}{|c|c|c|c|c|c|c|c|c|}
\hline Importer & Point & & & & & & & Total \\
\hline seller & of & & & Nov & per & Dec & per & Avg. \\
\hline Purchaser/End User & Entry & Vol & Price & Vol. & Price & Vol. & Price & Price \\
\hline
\end{tabular}

KIMBALL ENERGY CORPORATION Canadian Hydrocarbons Marketing Development Associates

KIMBALL ENERGY CORPORATION Canadian Hydrocarbons Marketing Greeley Gas Company

KIMBALL ENERGY CORPORATION Canadian Hydrocarbons Marketing IGI Resources

KIMBALL ENERGY CORPORATION Canadian Hydrocarbons Marketing Kimball Energy Corporation

KIMBALL ENERGY CORPORATION Canadian Hydrocarbons Marketing Kimball Energy Corporation

KIMBALL ENERGY CORPORATION Canadian Hydrocarbons Marketing North Canadian Marketing Corp.

KIMBALL ENERGY CORPORATION Canadian Hydrncarbons Marketing Pacific Gas \& Electric Company

KIMBALL ENERGY CORPORATION Canadian Hydrocarbons Marketing Portland General Electric Company
Sumas, Washington

Sumas, Washington

14

1.61

1.75

$0 \quad$ N/A

46

1.71

Sumas, Washington

325

1.54

231

1.98

395

2.25

952

1.94

Eastport, Idaho

1.60

$0 \quad$ N/A

0

N/A

49

1.60
Eastport, Idaho

$0 \quad N / A$

$0 \quad$ N/A

31

1.62 


\section{SHORT-TERM SALES}

4th Quarter 1993

Estinated Volumes (MMCF) \& Prices (\$/MMBTU) -- cont.

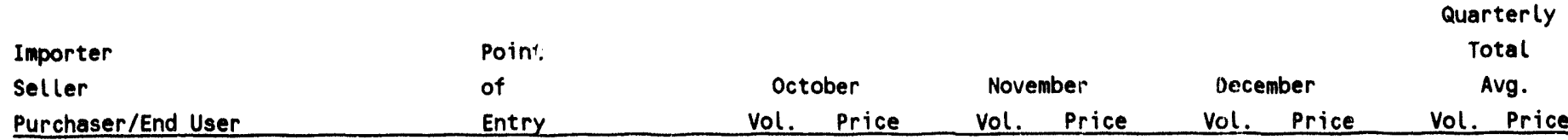

KIMBALL ENERGY CORPORATION Canadian Hydrocarbons Marketing Puget Sound Power \& Light

KIMBALL ENERGY CORPORATION Canadian Hydrocarbons Marketing Sierra Pacific Power Company

KIMBALL ENERGY CORPORATION Canadian Hydrocarbons Marketing Sierra Pacific Power Company

KIMBALL ENERGY CORPORATION Canadian Hydrocarbons Marketing Southwest Gas Corporation

KIMBALL ENERGY CORPORATION Canadian Hydrocarbons Marketing Tarrant Resources

KIMBALL ENERGY CORPORATION Canadian Hydrocarbons Marketing Tarrant Resources

KIMBALL ENERGY CORPORATION Canadian Hydrocarbons Marketing Utah Gas Services

KIMBALL ENERGY CORPORATION Canadian Hydrocarbons Marketing Wyoming Industrial Gas
Eastport, Idaho

$0 \quad N / A$

$0 \quad$ N/A

$5 \quad 1.79$

5

1.79

Eastport, Idaho

$0 \quad$ N/A

O N/A

11

1.75

$11 \quad 1.75$

Sumas, Washington

$92 \quad 1.49$

O N/A

O N/A

$92 \quad 1.49$

Sumas, Washington

O N/A

O N/A

$8 \quad 1.90$

$8 \quad 1.90$

Eastport, Idaho

O N/A

$0 \quad N / A$

$49 \quad 1.92$

$49 \quad 1.92$

Sumas, Hashington

O N/A

- N/A

$35 \quad 1.90$

35

1.90
$6 \quad 1.73$

o N/A

0

$N / A$

6

1.73 


$$
\text { Page - } 103
$$

\section{SHORT-TERM SALES}

4th Quarter 1993

Estimated Volumes (MMCF) \& Prices ( $\$ / M M B T U$ ) - cont.

Quarterly

Total

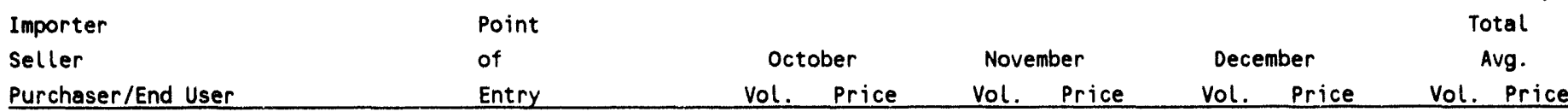

LOUIS DREYFUS ENERGY CORPORATION Canadian Natural Resources Limited Coastal Gas Marketing Company Sumas, Washington $\quad 0 \quad$ N/A

O N/A

$72 \quad 2.60$

$72 \quad 2.60$

LOUIS DREYFUS ENERGY CORPORATION Tarpon Gas Marketing Ltd.

DGS Trading

Niagara Falls, NY $124 \quad 1.84$

$0 \quad N / A$

$0 \quad$ N/A

124

1.84

LOUIS DREYFUS ENERGY CORPORATION Tarpon Gas Marketing Ltd.

National Fuel Resources

Niagara Falls, NY $\quad 40 \quad 1.84$

$0 \quad$ N/A

O N/A

$40 \quad 1.84$

LOUIS DREYFUS ENERGY CORPORATION Tarpon Gas Marketing Ltd.

Northern Consolidated Power Inc.

Niagara Falls, NY $265 \quad i .84$

$0 \quad$ N/A

$0 \quad N / A$

$265 \quad 1.84$

MADISON GAS \& ELECTRIC COMPANY Unigas Corporation

Madison Gas \& Electric

Port of Morgan, MT

O N/A

$30 \quad 1.97$

31

2.49

60

2.23

METROPOLITAN UTILITIES DISTRICT

Unigas Corporation

Metropolitan Utilities

Port of Morgan, MT $0 \quad$ N/A

$222 \quad 1.98$

195

2.53

416

2.24

MIDWEST GAS COMPANY

Unigas Corporation

Midwest Gas

Port of Morgan,

$0 \quad N / A$

$194 \quad 1.98$

202

2.49

397

2.24

MINNEGASCO

Unigas Corporation

Minnegasco

Port of Morgan, MT 0 N/A

$444 \quad 1.98$

462

2.49

906

2.24 


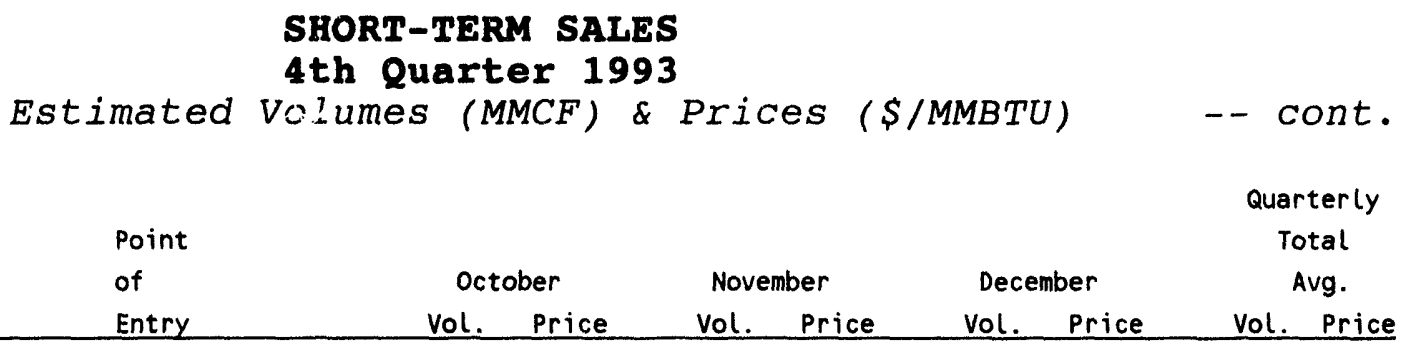

\section{MINNEGASCO}

Western Gas Marketing Limited

Minnegasco

Noyes, Minnesota

O N/A

355

2.20

366

2.50

721

2.35

MOBIL NATURAL GAS INC.

Mobil Oil Canada Ltd.

Brooklyn Interstate Natural Gas

Sumas, Washington

O N/A

$300 \quad 1.80$

$0 \quad$ N/A

$300 \quad 1.80$

MOBIL NATURAL GAS INC.

Mobil Oil Canada Ltd.

Cascade Natural Gas

Sumas, Washington

$0 \quad$ N/A

$549 \quad 1.80$

549

2.40

1098

2.10

MOBIL NATURAL GAS INC.

Mobil Oil Canada Ltd.

rcuastal Gas Marketing Company

Sumas, Washington

O N/A

$0 \quad N / A$

390

2.40

390

2.40

MOBIL NATURAL GAS INC. Mobil Oil Canada Ltd.

IGI Resources

Sumas, Washington $\quad 0 \quad$ N/A

$30 \quad 1.80$

1025

2.40

1055

2.38

MOBIL NATURAL GAS INC. Mobil Oil Canada Ltd. Mobil Natural Gas Inc.

MOBIL NATURAL GAS INC. Mobil Oil Canada Ltd. Mock Resources

MOBIL NATURAL GAS INC. Mobil Oil Canada Ltd. Natural Gas Resources 
Page -105

SHORT-TERM SALES

4th Quarter 1993

Estimated Volumes (MMCF) \& Prices (\$/MMBTU) -- cont.

\begin{tabular}{|c|c|c|c|c|c|c|}
\hline Importer & Point & & & & & $\begin{array}{c}\text { Quarterly } \\
\text { Total }\end{array}$ \\
\hline Purchaser/End User & Entry & Vol. Price & Price & Vol. & Price & Vol. Price \\
\hline
\end{tabular}

MOBIL NATURAL GAS INC.

Mobil Oil Canada Ltd.

Northern Minnesota Utilities

Noyes, Minnesota

$0 \quad N / A$

360

1.79

372

2.30

732

2.05

MOBIL NATURAL GAS INC.

Mobil Oil Canada Ltd.

Portland General Electric Company

Sumas, Washington

$0 \quad$ N/A

$150 \quad 1.80$

488

2.40

638

2.26

MOBIL NATURAL GAS INC.

Mobil Oil Canada Ltd.

Puget Sound Power \& Light

Sumas, Washington

O N/A

O N/A

10

2.40

10

2.40

MOBIL NATURAL GAS INC.

Mobil Oil Canada Ltd.

Sierra Pacific Power Company

Sumas, Washington

$0 \quad N / A$

$0 \quad N / A$

41

2.40

$41 \quad 2.40$

MOBIL NATURAL GAS INC.

Mobil Oil Canada Ltd.

Southwest Gas Corporation

Sumas, Washington

$0 \quad N / A$

150

1.80

126

2.40

276

2.07

MOBIL NATURAL GAS INC. Mobil Oil Canada Ltd.
Union Pacific Fuels

MOBIL NATURAL GAS INC. Mobil Oil Canada Ltd. Union Pacific Resources

$433 \quad 1.80$

$0 \quad N / A$

$0 \quad N / A$

$433 \quad 1.80$

Sumas, Washington

$0 \quad N / A$

$438 \quad 1.80$

464

2.40

901

2.11

MOBIL NATURAL GAS INC.

Mobil Oil Canada Ltd.

Washington Natural Gas Company

Sumas, Washington

N/A

$240 \quad 1.80$

205

2.40

445

2.08 


$$
\text { Page - } 106
$$

\begin{tabular}{cccc}
\multicolumn{4}{c}{ SHORT-TERM SALES } \\
4th Quarter 1993
\end{tabular}

Importer

Entry

Vol.

Vol. Price

Vol. Price

Vol. Price

MOBIL NATURAL GAS INC.

Mobil Oil Canada Ltd.

Washington Water Power Company

Sumas, Washington

$0 \quad N / A$

$0 \quad$ N/A

90

2.40

90

2.40

MOCK RESOURCES, INC.

Direct Energy Marketing Limited

Sierra Pacific Power Company

Sumas, Washington

$0 \quad N / A$

$12 \quad 1.82$

$0 \quad N / A$

12

1.82

MOCK RESOURCES, INC.

Direct Energy Marketing Limited

Washingion Natural Gas Company

Sumas, Washington

$0 \quad N / A$

$0 \quad$ N/A

3

2.06

3

2.06

MOCK RESOURCES, INC.

Inland Natural Gas

IGI Resources

Sumas, Washington

O N/A

$26 \quad 1.96$

$0 \quad N / A$

$26 \quad 1.96$

MOCK RESOURCES, INC.

Inland Natural Gas

Northwest Natural Gas Company

Sumas, Washington

$0 \quad N / A$

$32 \quad 1.96$

30

2.31

62

2.13

MOCK RESOURCES, INC.

Inland Natural Gas

Sierra Pacific Power Company

Sumas, Washington $\quad 0 \quad N / A$

$20 \quad 1.96$

$31 \quad 2.31$

51

2.17

MOCK RESOURCES, INC.

Inland Natural Gas

Southern California Gas Company

Sumas, Washington $\quad 16 \quad 1.64$

$0 \quad N / A$

$0 \quad$ N/A

16

1.64

MOCK RESOURCES, INC.

Talisman

Pacific Gas \& Electric Company

Sumas, Washington $\quad 0 \quad$ N/A

$5 \quad 1.78$

$0 \quad N / A$

5

1.78 


$$
\text { Page - } 107
$$

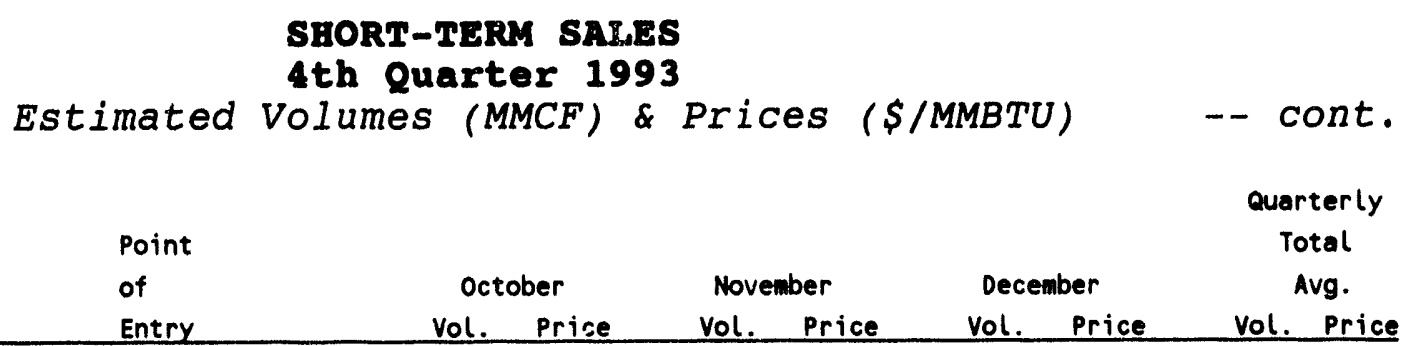

MOCK RESOURCES, INC.

Westcoast Energy Marketing, Ltd.

Sierra Pacific Power Company

Sumas, Washington

$0 \quad$ N/A

$0 \quad$ N/A

$60 \quad 1.88$

$60 \quad 1.88$

MURPHY GAS GATHERING

Murphy Oil Company Ltd.

Murphy Gas Gathering

Noyes, Minnesot

O N/A

$97 \quad 1.91$

101

2.19

198

2.05

NATIONAL STEEL CORPORATION

Direct Energy Marketing Limited Notional Steel

Detroit, Michigan

$153 \quad 1.64$

O N/A

$0 \quad N / A$

$153 \quad 1.64$

NATIONAL STEEL CORPORATION Enron Gas Marketing Canada National Steel

Detroit, Michigan

$0 \quad$ N/A

148

2.74

153

3.01

301

2.88

NATIONAL STEEL CORPORATION Unigas Corporation National Steel

Detroit, Michigan $346 \quad 1.70$

O N/A

O N/A

346

1.70

NATIONAL STEEL CORPORATION West Coast Gas Services Inc. National Steel

NATURAL GAS CLEARINGHOUSE INC. Alberta \& Southern Company Ltd. Northwest Natural Gas Company

NATURAL GAS CLEARINGHOUSE INC. Alberta \& Southern Company Ltd. Pacific Gas \& Electric Company 
Page - 108

SHORT-TERM SALES
4th Quarter 1993
Estimated Volumes (MMCF) \& Prices (\$/MMBTU)

Importer

seller

Purchaser/End User

\section{Entry}

Vol. Price

Vol. Price

Sumas, Washington

O N/A

$18 \quad 2.04$

O N/A

18

2.04

NATURAL GAS CLEARINGHOUSE INC.

Dekalb Energy Canada Ltd.

Washington Natural Gas Company

NATURAL GAS CLEARINGHOUSE INC. Inland Natural Gas

Development Associates

NATURAL GAS CLEARINGHOUSE INC.

Inland Natural Gas

IGI Resources

Sumas, Washington

$22 \quad 1.67$

$0 \quad N / A$

O N/A

22

1.67

NATURAL GAS CLEARINGHOUSE INC. Inland Natural Gas

Northwest Natural Gas Company

Sumas, Washington

29

1.67

$0 \quad N / A$

$0 \quad$ N/A

29

1.67

NATURAL GAS CLEARINGHOUSE INC. Inland Natural Gas

Oregon Natural Gas Development

Sumas, Washington $\quad 14 \quad 1.67$

O N/A

$0 \quad$ N/A

14

1.67

NATURAL GAS CLEARINGHOUSE INC. Inland Natural Gas

Pacific Gas Transmission

Sumas, Washington

$18 \quad 1.67$

O N/A

O N/A

18

1.67

NATURAL GAS CLEARINGHOUSE INC. Mobil Oil Canada Ltd.

Northwest Natural Gas Company
Sumas, Washington $\quad 16 \quad 1.67$

D N/A

$0 \quad$ N/A

16

1.67 


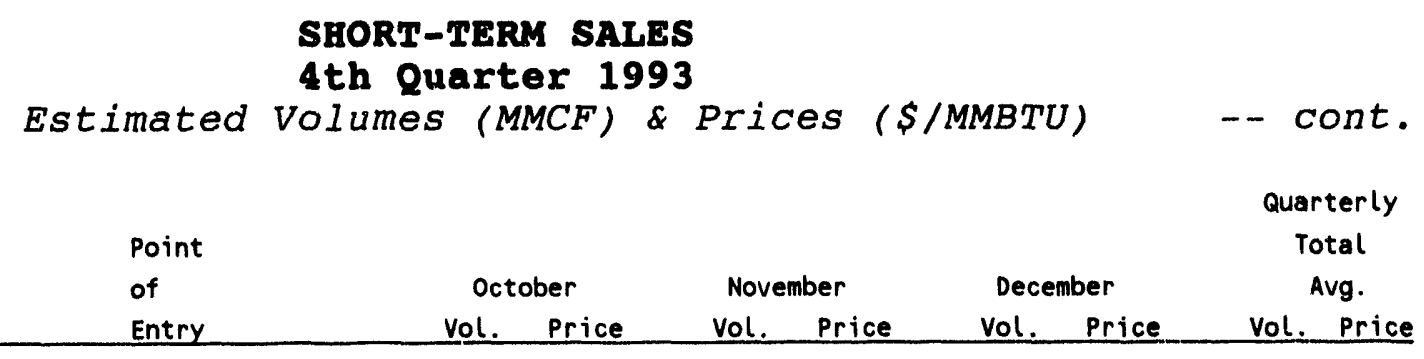

NATURAL GAS CLEARINGHOUSE INC. Mobil Oil Canada Ltd.

Oregon Natural Gas Development Sumas, Washington $\quad 10 \quad 1.67$

$0 \quad$ N/A

$0 \quad$ N/A

$10 \quad 1.67$

NATURAL GAS CLEARINGHOUSE INC.

Mobil Oil Canada Ltd.

Pacific Gas Transmission

Sumas, Washington $\quad 181 \quad 1.67$

$0 \quad N / A$

$0 \quad$ N/A

181

1.67

NORTH AMERICAN RESOURCES COMPANY Altana Exploration

North American Resources Company

Babb, Montana

$110 \quad 1.60$

$52 \quad 1.99$

135

1.83

297

1.77

NORTH CANADIAN MARKETING CORPORATION Alberta \& Southern Company Ltd.

Enron Gas Marketing

NORTH CANADIAN MARKETING CORPORATION Alberta \& Southern Company Ltd. National Gas Resources

NORTH CANADIAN MARKETING CORPORATION Alberta \& Southern Company Ltd. Southern California Gas Company

NORTH CANADIAN MARKETING CORPORATION Alberta \& Southern Company Ltd. Sunrise Energy 
Page - 110

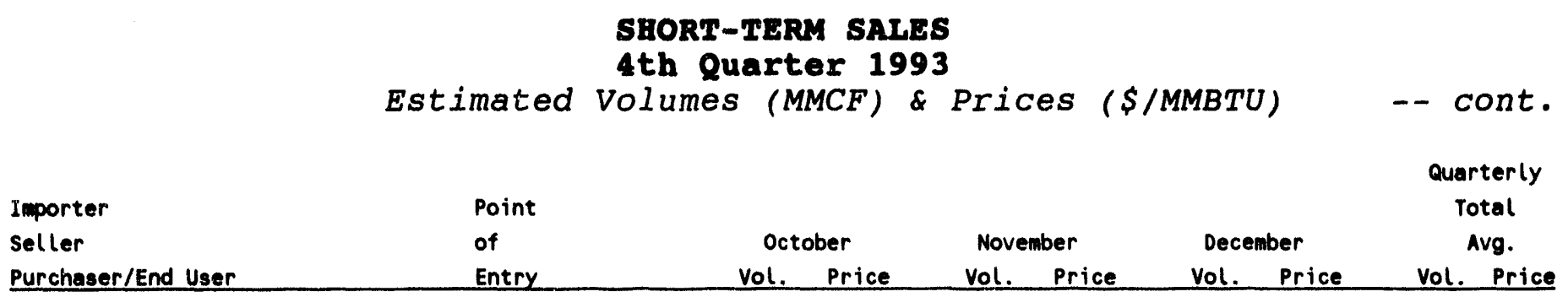

MCATH CANADIAN MARKETING CORPORATION

North Canadian Marketing Inc.

Enron Gus Marketing

Eastport, Idaho

O N/A

0 N/A

$2 \quad 2.23$

$2 \quad 2.23$

NORTH CANADIAN MARKETING CORPORATION North Canadian Marketing Inc.

Mock Resources

Eastport, Idaho

$0 \quad N / A$

241

1.94

218

2.23

459

2.08

NORTH CANADIAN MARKETING CORPORATION North Canadian Marketing Inc.

National Gas Resources

Eastport, Idaho

$0 \quad$ N/A

$288 \quad 1.94$

O N/A

$288 \quad 1.94$

NORTH CANADIAN MARKETING CORPORATION North Canadian Marketing Inc.

Natural Gas Clearinghouse

Eastport, Idaho

O N/A

$0 \quad N / A$

71

2.23

71

2.23

NORTH CANADIAN MARKEIING CORPORATION North Canadian Marketing Inc.

North Canadian Marketing Corp.

Noyes, Minnesota

$223 \quad 1.94$

$216 \quad 2.05$

223

2.11

662

2.03

NORTH CANADIAN MARKETING CORPORATION North Canadian Marketing Inc.

North Canadian Marketing Corp.

Port of Morgan, MT 0 N/A

$0 \quad N / A$

427

1.70

427

1.70

NORTH CANADIAN MARKETING CORPORATION North Canadian Marketing Inc.

Northern Illinois Gas

Port of Morgan, MT 2/88 1.20

48

2.14

O N/A

295

1.35

NORTH CANADIAN MARKETING CORPORATION North Canadian Marketing Inc. 
Page - 111

\section{SEORT-TERY SALES}

4th Quarter 1993

Estimated Volumes (MMCF) \& Prices (\$/MMBTU) -- cont.

Importer Point

Seller of

Purchaser/End User

Entry

October

vol. Price

November

Vol. Price

December

Vol. Price

Quarterly

Total

Avg.

Vol. Price

NORTH CANADIAN MARKETING CORPORATION North Canadian Marketing Inc. PGME UEG

Eastport, Idaho

$0 \quad N / A$

$0 \quad N / A \quad 1134$

2.23

1134

2.23

MORTH CAMADIAN MARKETING CORPORATION North Canadian Marketing Inc.

Sunrise Energy

$0 \quad N / A$

1319

1.94

O N/A

1319

1.94

MORTH CANADIAN MARKETING CORPORATION North Canadian Marketing Inc.

Tenaska Marketing Ventures

Port of Morgan, MT $303 \quad 1.20$

O N/A

102

1.70

405

1.33

MORTH CAMADIAN MARKETING CORPORATION North Canadian Marketing Inc.

Tosco Energy

Eastport, Idaho

$0 \quad N / A$

$27 \quad 1.94$

53

2.23

80

2.13

MORTH CAMADIAN MARKETIMG CORPORATION Morth Canadian Marketing Inc. Western Gas Marketing Inc.

MORTHERN ILLINOIS GAS COMPANY Unigas Corporation Northern Illinois Gas

2.70

177

NORTHEAN ILLIMOIS GAS COMPANY Western Gas Marketing Linited Northern Illinois Gas

Noyes, Minnesota

$0 \quad N / A$

91

2.14

94

2.44

186

2.29

MORTHERN MINWESOTA UTILITIES Proces Linited

Northern Minnesota Utilities

Noyes, Minnesota

15

1.86

242

2.21

271

2.14

669

2.10 


$$
\text { Page - } 112
$$

ATTACHMENT $C-4$

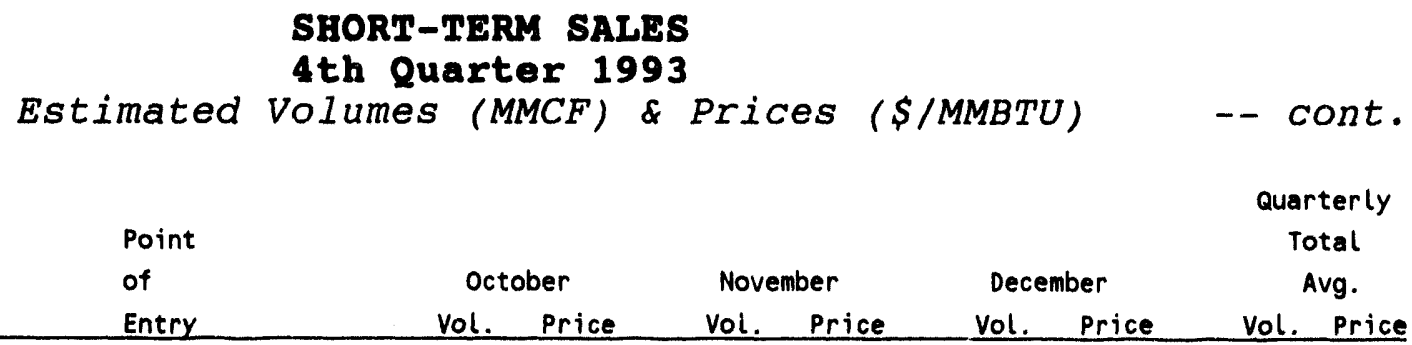

NORTHERN MINNESOTA UTILITIES

West Coast Gas Services Inc.

Northern Minnesota Utilities

$\begin{array}{lllll}\text { Port of Morgan, MT } & 0 & \text { N/A } & 14 & 1.99\end{array}$

$6 \quad 2.82$

21

2.25

NORTHERN MINNESOTA UTILITIES

Western Gas Marketing Limited

Northern Minnesota Utilities

Noyes, Minnesota

O N/A

226

2.25

233

2.51

459

2.38

NORTHERN STATES POWER COMPANY (MINNESOTA)

Unigas Corporation

Northern States Power (MN)

Port of Morgan, MT

N/A

189

1.98

207

2.51

396

2.26

NORTHERN STATES POWER COMPANY (WISCONSIN)

Unigas Corporation

Northern States Power (WI)

Port of Morgan, MT

$0 \quad N / A$

$35 \quad 1.98$

19

2.51

54

2.17

NORTHERN STATES POWER COMPANY (MINNESOTA)

Western Gas Marketing Limited

Northern States Power (MN)

Noyes, Minnesota

$0 \quad N / A$

398

2.18

407

2.56

805

2.37

NORTHERN STATES POWER COMPANY (WISCONSIN)

Western Gas Marketing Limited

Northern States Power (WI)

Noyes, Minnesota $\quad 0 \quad$ N/A

$74 \quad 2.18$

76

2.56

150

2.37

NORTHERN UTILITIES, INC.

Renaissance Energy Ltd.

Northern Utilities, Inc.

Niagara Falls, NY $0 \quad$ N/A

29

3.17

29

3.57

58

3.37

NORTHRIDGE GAS MARKETING, INC.

Northridge Petroleum Marketing, Inc.

Asarco Inc.

Babb, Montana

$25 \quad 1.94$

$29 \quad 1.94$

$56 \quad 1.94$

110

1.94 


SHORT-TERM SALES
4th Quarter 1993
Estimated Volumes (MMCF) \& Prices (\$/MMBTU)

NORTHRIDGE GAS MARKETING, INC.

Northridge Petroleum Marketing, Inc.

Barretts Minerals

Babb, Montan

$20 \quad 1.95$

$39 \quad 1.95$

$27 \quad 1.95$

$86 \quad 1.95$

NORTHRIDGE GAS MARKETING, INC.

Northridge Petroleum Marketing, Inc

cibola corporation

Port of Morgan, MT $92 \quad 1.72$

$1 \quad 1.88$

$0 \quad$ N/A

$93 \quad 1.72$

NORTHRIDGE GAS MARKETING, INC.

Northridge Petroleum Marketing, Inc.

Columbia Falls

Babb, Montana

$17 \quad 1.95$

$21 \quad 1.95$

22

1.95

60

1.95

NORTHRIDGE GAS MARKETING, INC.

Northridge Petroleum Marketing, Inc.

Holman

Babb, Montana

126

1.59

O N/A

$0 \quad N / A$

126

1.59

NORTHRIDGE GAS MARKETING, INC.

Northridge Petroleum Marketing, Inc.

Interenergy Corporation

Babb, Montana

$41 \quad 1.33$

$0 \quad$ N/A

$84 \quad 1.82$

125

1.66

NORTHRIDGE GAS MARKETING, INC.

Northridge Petroleum Marketing, Inc.

John Brown oil \& Gas

Babb, Montana

$72 \quad 1.34$

$0 \quad N / A$

$6 \quad 1.95$

$78 \quad 1.39$

NORTHRIDGE GAS MARKETING, INC.

Northridge Petroleum Marketing, Inc.

Louisiana Pacific

Babb, Montana

$18 \quad 1.95$

$28 \quad 1.95$

15

1.95

$61 \quad 1.95$

NORTHRIDGE GAS MARKETING, INC.

Northridge Petroleum Marketing, Inc.

Northridge Petroleum Marketing U.S. Noyes, Minnesota

32

2.03

$33 \quad 2.00$

23

2.05

88

2.02 


$$
\text { Page - } 114
$$

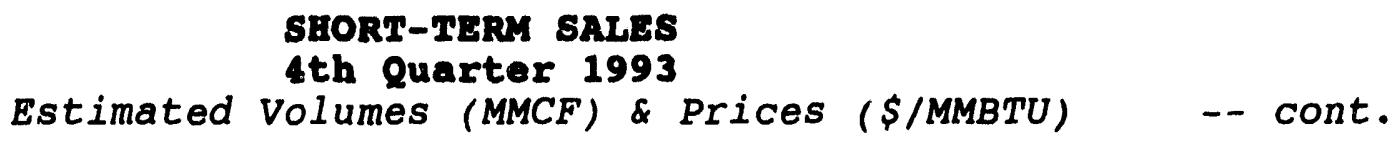

\begin{tabular}{|c|c|c|c|c|c|}
\hline $\begin{array}{l}\text { Importer } \\
\text { seller }\end{array}$ & $\begin{array}{l}\text { Point } \\
\text { of }\end{array}$ & October & November & Decenber & $\begin{array}{c}\text { Total } \\
\text { Avg. }\end{array}$ \\
\hline Purchaser/End User & Entry & Vol. Price & Vol. Price & Vol. Price & Vol. Pric \\
\hline
\end{tabular}

MORTHRIDGE GAS MARKETING, INC.

Northridge Petroleum Marketing, Inc.

Plum Creek Manufacturing

NORTHRIDGE GAS MARKETING, INC.

Northridge Petroleun Marketing, Inc.

Rhone-Poulenc

Babb, Montana

$31 \quad 1.95$

$48 \quad 1.95$

49

1.95

128

1.95

MORTHRIDGE GAS MARKETING, IMC.

Northridge Petroleum Marketing, Inc.

stone Container Corporation

Babb, Montana

$60 \quad 1.55$

135

1.98

175

1.98

370

1.91

MORTHRIDGE GAS MARKETIMG, IMC.

Northridge Petroleum Marketing, Inc.

Tenaska Marketing Ventures

Port of Morgan, ITT $51 \quad 1.81$

$0 \quad N / A$

$0 \quad$ N/A

51

1.81

MORTHRLOGE GAS MARKETIAG, INC.

Northridge Petroleum Marketing, Inc.

Union Gas Linited

Noyes, Minnesota

$483 \quad 2.03$

481

2.00

476

2.05

1440

2.03

MORTHUESTERM PUBLIC SERVICE COMPANY

Unigas Corporation

Northwestern Public Service Company

Port of Morgan, MT

$0 \quad$ N/A

$28 \quad 1.97$

$29 \quad 2.53$

58

OREGON MATURAL GAS DEVELOPMENT CORPORATION

Canstates Gas Marketing

Oregon Matural Gas Development

Eastport, Idaho

$55 \quad 1.65$

O N/A

o N/A

$55 \quad 1.65$

ORYX GAS MARKETING, L.P.

PEMEX

Oryx Gas Marketing Company

Hidalgo, Texas

$0 \quad N / A$

O N/A

86

2.05

86

2.05 


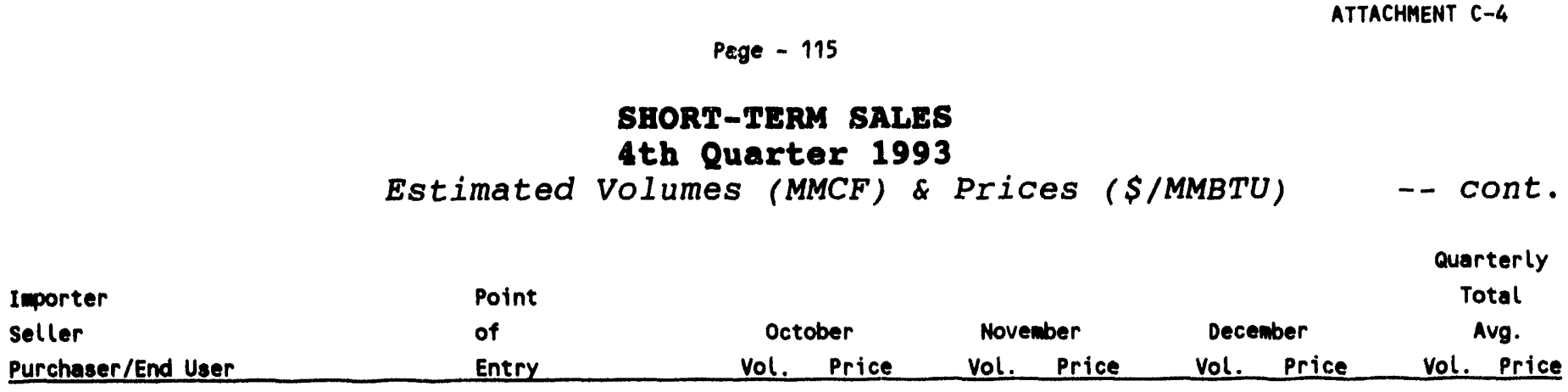

PACIFIC GAS TRANSMISSION COMPANY

Western Gas Marketing Limited

Pacific Gas Transwission (Linepack) Eastport, Idaho $\quad \begin{array}{llllllll}98 & 1.85 & 48 & 1.95 & 0 & \text { N/A } & 146 & 1.88\end{array}$

PAN MATIONAL GAS SALES, INC.

Sonatrading

Panhandle Trading Company

Lake Charles, LA $166 \quad 1.83 \quad 71 \quad 1.76$

$44 \quad 1.76$

$281 \quad 1.80$

PAN-ALBERTA GAS (U.S.), INC.

Northwest Pacific Energy Marketing Inc.

Arco Products Company

Sumas, Washington $105 \quad 1.39 \quad 136 \quad 1.93$

$91 \quad 2.15$

$331 \quad 1.82$

PAN-ALBERTA GAS (U.S.), INC.

Northwest Pacific Energy Marketing Inc.

James River Corporation

Sumas, Washington

328

$1.39 \quad 318 \quad 1.93$

443

2.15

$1089 \quad 1.86$

PAN-ALBERTA GAS (U.S.), INC.

Northwest Pacific Energy Marketing Inc.

Matural Gas Clearinghouse

Sumas, Mashington

$0 \quad$ N/A

1336

1.93

1619

2.15

2955

2.05

PAN-ALBERTA GAS (U.S.), IMC.

Morthwest Pacific Energy Marketing Inc.

Northwest Pipeline

Sumas, Washington

885

1.39

805

1.93

406

2.15

2096

1.74

PAN-ALBERTA GAS (U.S.), INC.

Northwest Pacific Energy Marketing Inc.

Pacific Gas a Electric Comany

Sumas, Hashington

O N/A

$363 \quad 1.93$

O N/A

$363 \quad 1.93$

PAN-ALBERTA GAS (U.S.), INC.

Pan-Alberta Gas Ltd.

Matural Gas Clearinghouse

Eestport, Idaho

$0 \quad N / A$

O N/A

20

1.83

20

1.83 


SHORT-TERM SALES
4th Quarter 1993
Estimated Volumes (MMCF) \& Prices (\$/MMBTU;

$\begin{array}{ll}\text { Importer } & \text { Point } \\ \text { Seller } & \text { of }\end{array}$

vol. Price

Vol. Price

Vol. Price

Vol. Price

PAN-ALBERTA GAS (U.S.), INC.

Pan-Alberta Gas Ltd.

Pacific Gas \& Electric Company

Eastport, Idaho

O N/A

$363 \quad 1.58$

577

1.83

940

1.73

PANHANDLE TRADING COMPANY

PEMEX

Panhandle Trading Company

Hidalgo, Texas

$0 \quad N / A$

$0 \quad$ N/A

160

1.80

160

1.80

PARAMOUNT RESOURCES U.S., INC.

Paramount Resources Ltd.

cook Inlet

Eastport, Idaho

$0 \quad$ N/A

589

1.10

607

1.63

1196

1.37

PAWTUCKET POWER ASSOCIATES L.P.

Pawtucket Power Associates

O \& R Energy

Waddington, NY

$19 \quad 2.20$

$4 \quad 2.56$

$0 \quad N / A$

23

2.26

PEOPLES NATURAL GAS COMPANY Unigas Corporation

Peoples Natural Gas Company

Port of Morgan, MT

$0 \quad N / A$

$240 \quad 1.73$

180

2.26

420

1.96

PEOPLES NATURAL GAS COMPANY Western Gas Marketing Limited

Peoples Natural Gas Company

Noyes, Minnesota

$0 \quad N / A$

186

2.16

194

2.45

380

2.31

PEPPERELL POWER ASSOCIATES L.P. SP

Western Gas Marketing Limited

Nizgara falls, NY 32

$11 \quad 2.25$

$0 \quad N / A$

43

2.15

PETRO-CANADA HYDROCARBONS, INC.

Petro-Canada Hydrocarbons Inc.

Bridgegas

Sumas, Washington $\quad 0 \quad$ N/A

$10 \quad 1.78$

$0 \quad N / A$

$10 \quad 1.78$

SP - Inter ruptible/special purchase sale made under long-term authorization 
Page - 117

\begin{tabular}{|c|c|c|c|c|c|}
\hline & & $\begin{array}{l}\text { SHORT-TERM SALES } \\
\text { 4th Quarter } 199\end{array}$ & & & \\
\hline & Estimated & Volumes $(M M C F) \&$ & Prices ( & $(\$ / M M B T U)$ & - cont. \\
\hline Importer & Point & & & & $\begin{array}{l}\text { Quarterly } \\
\text { Total }\end{array}$ \\
\hline Seller & of & October & November & December & Avg. \\
\hline Purchaser/End User & Entry & Vol. Price & Vol. Price & Vol. Price & Vol. Price \\
\hline
\end{tabular}

PETRO-CANADA HYDROCARBONS, INC. Petro-Canada Hydrocarbons inc. Coastal Gas Marketing Company

PETRO-CANADA hYDROCARBONS, INC. Petro-Canada Hydrocarbons Inc. Grand Valley Gas Company

PETRO-CANADA HYDROCARBONS, INC. Petro-Canada Hydrocarbons Inc. IGI Resources

PETRO-CANADA HYDROCARBONS, INC. Petro-Canada Hydrocarbons Inc. Intalco Aluminum Corporation

PETRO-CANADA HYDROCARBONS, INC. Petro-Canada Hydrocarbons Inc.

PETRO-CANADA HYDRUCARBONS, INC. Petro-Canada Hydrocarbons Inc.

J. Aaron Company

PETRO-CANADA HYOROCARBONS, INC. Petro-canada Hydrocarbons Inc. Natural Gas Clearinghouse

PETRO-CANADA HYDROCARBONS, INC. Petro-Canada Hydrocarbons Inc. 


$$
\text { Page - } 118
$$

\section{SHORT-TERM SALES \\ 4th Quarter 1993}

Estimated Volumes (MMCF) \& Prices (S/MMBTU) -- cont.

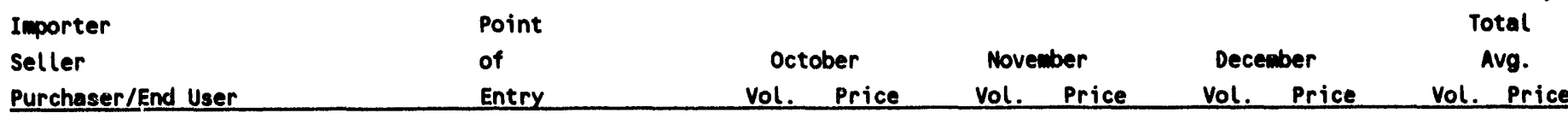

PETRO-CANADA HYDROCARBONS, INC.

Petro-Canada Hydrocarbons Inc.

Sierra Pacific Power Company

Sumas, Washington $\quad 0 \quad N / A$

435

1.78

$449 \quad 2.31$

884

2.05

PETRO-CANADA hYDROCAREOHS, INC. Petro-Canada Hydrocarbons Inc.

Washington Matural Gas Company Sumas, Washington

$299 \quad 1.50$

$290 \quad 1.78$

299

2.31

889

1.86

PGRE GAS SUPPLY BUSINESS UNIT

AEC Oil Gas Company

PGSE Gas Supply Business Unit

Eastport, Idaho

O N/A

$274 \quad 1.86$

464

2.27

738

2.12

PGRE GAS SUPPLY QUSINESS UNIT Amerada Hess Canado

PGRE Gas Supply Business Unit

Eastport, Idaho

O N/A

399

2.07

337

2.37

736

2.21

PGEE GAS SUPPLY BUSINESS UNIT American Hunter Exploration Ltd. PGeE Gas Supply Business Unit

Eastport, Idaho

O N/A

$439 \quad 1.71$

1.58

459

1.70

PGEE GAS SUPPLY DUSINESS UNIT Anoco Canada Marketing Corporation PGRE Gas Supply Business Unit

Eastport, Idaho

O N/A

O N/A

$19 \quad 1.95$

19

1.95

PGEE GAS SUPPLY BUSINESS UNIT Amoco Canada Petroleum Company Ltd. PCEE Gas Supply Business Unit

Eastport, Idaho

o N/A

253

1.89

1647

2.49

4186

2.12

PGE GAS SUPPLY BUSIMESS UNIT Anderson Exploration Ltd.

PGeE Gas Supply Business Unit

Eastport, Ideho

o M/A

1643

2.14

1388

2.43

3031

2.27 


SHORT-TERM SALES
4th Quarter 1993
Estimated Volumes (MMCF) \& Prices (S/MMBTU)

PGBE GAS SUPPLY BUSINESS UNIT Brywo Energy, Inc.

PG8E Gas Supply Business Unit

Eastport, Idaho

O N/A

$293 \quad 1.75$

$0 \quad$ N/A

$293 \quad 1.75$

PG\&E GAS SUPPLY BUSINESS UNIT C \& B Production

PG\&E Gas Supply Business Unit

Eastport, Idaho

$0 \quad N / A$

$361 \quad 1.76$

$0 \quad$ N/A

361

1.76

PG\&E GAS SUPPLY BUSINESS UNIT CanWest Gas Supply Inc.

PG\&E Gas Supply Business Unit

Eastport, Idaho

$0 \quad$ N/A

$146 \quad 1.82$

N/A

146

1.82

PGRE GAS SUPPLY BUSINESS UNIT Canadian Hunter Exploration Ltd. PG\&E Gas Supply Business Unit

Eastport, Idaho

$0 \quad N / A$

$941 \quad 1.94$

748

2.36

1689

2.13

PGRE GAS SUPPLY BUSINESS UNIT Canstates Gas Marketing

PG\&E Gas Supply Business Unit

Eastport, Idaho

$0 \quad$ N/A

346

2.08

246

2.30

592

2.17

PG\&E GAS SUPPLY BUSINESS UNIT Chevron Canada

PG\&E Gas Supply Business Unit

Eastport, Idaho

$0 \quad N / A$

$1285 \quad 1.84$

1669

2.18

2953

2.03

PG\&E GAS SUPPLY BUSINESS UNIT Cibola Canada

PG\&E Gas Supply Business Unit

Eastport, Idaho

$0 \quad$ N/A

29

2.37

155

1.60

184

1.72

PG\&E GAS SUPPLY BUSINESS UNIT Coastal Gas Marketing Company PG\&E Gas Supply Business Unit 


$$
\text { Page - } 120
$$

ATTACHMENT C-4

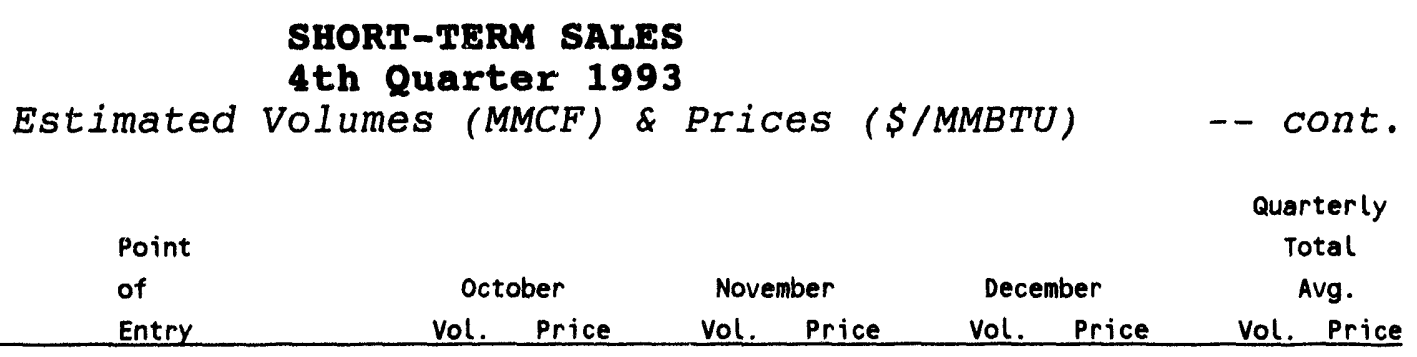

PG\&E GAS SUPPLY BUSINESS UNIT Colonial Oil \& Gas Limited PG\&E Gas Supply Business Unit Eastport, Idaho $0 \quad N / A$ 165 2.00 $153 \quad 2.27$

PG\&E GAS SUPPLY BUSINESS UNIT Cook Inlet Energy Supply PGRE Gas Supply Business Unit Eastport, Idaho $0 \quad$ N/A

$0 \quad N / A$

591

2.40

591

2.40

PGRE GAS SUPPLY BUSINESS UNIT Crestar Energy

PG\&E Gas Supply Business Unit

Eastport, Idaho

O N/A

166

2.31

875

2.11

1041

2.14

PG\&E GAS SUPPLY BUSINESS UNIT Direct Energy Marketing Limited PG\&E Gas Supply Business Unit

$\begin{array}{ll}78 & 2.24\end{array}$

736

2.02

815

2.04

PG\&E GAS SUPPLY BUSINESS UNIT Encore Energy Corporation PG\&E Gas Supply Business Unit

PG\&E GAS SUPPLY BUSINESS UNIT Enron Gas Marketing Canada PGBE Gas Supply Business Unit 


$$
\text { Page - } 121
$$

SHORT-TERM SALES
4th Quarter 1993
Estimated Volumes (MMCF) \& Prices (\$/MMBTU)

seller

Entry

Vol. Price

Vol. Price

Vol. Price

Vol. Price

PG\&E GAS SUPPLY BUSINESS UNIT Gulf Canada Resources Limited PG\&E Gas Supply Business Unit

Eastport, Idaho

$0 \quad$ N/A

33

2.02

564

2.18

897

2.12

PG\&E GAS SUPPLY BUSINESS UNIT Imperial oil

PG\&E Gas Supply Business Unit

Eastport, Idaho

O N/A

176

2.00

298

2.27

474

2.17

PG\&E GAS SUPPLY BUSINESS UNIT Institutional Capital Corporation PG\&E Gas Supply Business Unit

Eastport, Idaho

$0 \quad N / A$

1235

1.83

1090

2.40

2325

2.10

PG\&E GAS SUPPLY BUSINESS UNIT Mobil Natural Gas Inc.

PG\&E Gas Supply Business Unit

PG\&E GAS SUPPLY BUSINESS UNIT Mock Resources, Inc.

PG\&E Gas Supply Business Unit

PGBE GAS SUPPLY BUSINESS UNIT NGL Supply (Gas) Company Ltd. PG\&E Gas Supply Business Unit

PG\&E GAS SUPPLY BUSINESS UNIT Natural Gas Clearinghouse PG\&E Gas Supply Business Unit

Eastport, Idaho

D N/A

$8 \quad 1.97$

$0 \quad N / A$

$8 \quad 1.97$

PG\&E GAS SUPPLY BUSINESS UNIT Northridge Petroleum Marketing, Inc. PG\&E Gas Supply Business Unit Eastport, Idaho
$0 \quad N / A$

10

1.58

10

1.58
$0 \quad N / A$

229

2.02

$0 \quad N / A$

229

2.02

Eastport, Idaho

o N/A

332

1.98

300

2.31

632

2.14 


$$
\text { Page - } 122
$$

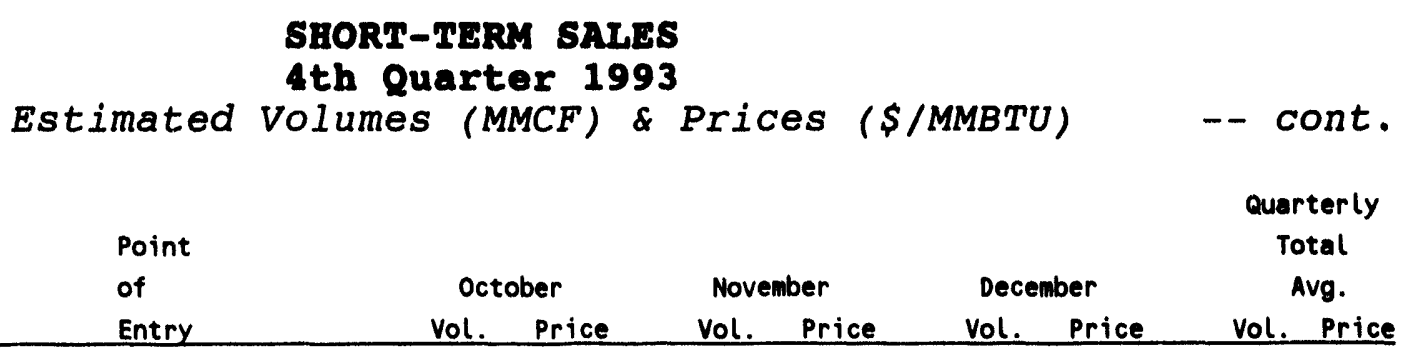

PG\&E GAS SUPPLY BUSINESS UNIT Pan-Alberta Gas Ltd.

PG\&E Gas Supply Business Unit

PG\&E GAS SUPPLY BUSINESS UNIT Perry Gas Companies, Inc.

PG\&E Gas Supply Business Unit

PGRE GAS SUPPLY BUSINESS UNIT Petro Source Gas Ventures PG\&E Gas Supply Business Unit

PGRE GAS SUPPLY BUSINESS UNIT Poco Petroleum Ltd.

PGBE Gas Supply Business Unit

PG8E GAS SUPPLY BUSINESS UNIT Premier Enterprises, Inc.

PG\&E Gas Supply Business Unit

PG\&E GAS SUPPLY BUSINESS UNIT Progas Limited

PG\&E Gas Supply Business Unit

PG\&E GAS SUPPLY BUSINESS UNIT RFA Natural Gas Inc.

PG\&E Gas Supply Business Unit

PG\&E GAS SUPPLY BUSINESS UNIT Shell Canada Limited

PG\&E Gas Supply Business Unit
Eastport, Idaho

O N/A

$367 \quad 2.01$

$530 \quad 1.99$

897

2.00

Eastport, Idaho

$0 \quad$ N/A

$293 \quad 1.88$

150

2.40

443

2.06

Eastport, Idaho

O N/A

$449 \quad 2.08$

1356

1.99

1806

2.01

Eastport, Idaho

$0 \quad N / A$

$44 \quad 1.97$

58

1.58

102

1.75

Eastport, Idaho

- N/A

O N/A

291

2.39

291

2.39

Eastport, Idaho

$0 \quad N / A$

107

2.20

470

2.00

577

2.04

Eastport, Idaho

$0 \quad$ N/A

881

1.79

903

2.31

1784

2.05

Eastport, Idaho

$0 \quad$ N/A

$898 \quad 2.02$

1513

2.28

2411

2.18 


$$
\text { Page - } 123
$$

\section{SHORT-TERM SALES \\ 4th Quarter 1993}

Estimated Volumes (MMCF) \& Prices ( $\$ / M M B T U$ ) -- cont.

Importer

Seller

Purchaser/End User
Point

of

Entry
Quarterly

Total

Avg.

PG\&E GAS SUPPLY BUSINESS UNIT Western Gas Marketing Limited PG\&E Gas Supply Business Unit

October

Vol. Price
November

Vol. Price
Eastport, Idaho

$0 \quad N / A$

$990 \quad 2.06$

815

December

Vol. Price
Vol. Price

POCO PETROLEUM, INC.

Poco Petroleum Ltd.

Bridgegas

Babb, Montana

$63 \quad 1.86$

O N/A

0 N/A

$63 \quad 1.86$

POCO PETROLEUM, INC.

Poco Petroleum Ltd.

Hadson Gas Systems

POCO PETROLEUM, INC.

Poco Petroleum Ltd.

IGI Resources

Eastport, Idaho

O N/A

$89 \quad 2.29$

142

2.01

232

2.12

POCO PETROLEUM, INC.

Poco Petroleum Ltd.

IGI Resources

Sumas, Washington

110

1.87

2177

2.13

$0 \quad N / A$

3281

2.04

POCO PETROLEUM, INC.

Poco Petroleum Ltd.

Northwest Natural Gas Company

Sumas, Washington $402 \quad 1.96$

570

2.32

620

2.24

1592

2.20

POCO PETROLEUM, INC.

Poco Petroleum Ltd.

Pacific Coastal Gas

POCO PETROLEUM, INC.

Poco Petroleum Ltd.

Pacific Gas \& Electric Company
Eastport, Idaho

$0 \quad$ N/A

$231 \quad 1.96$

$30 \quad 1.79$

261

1.94 


$$
\text { Page - } 124
$$

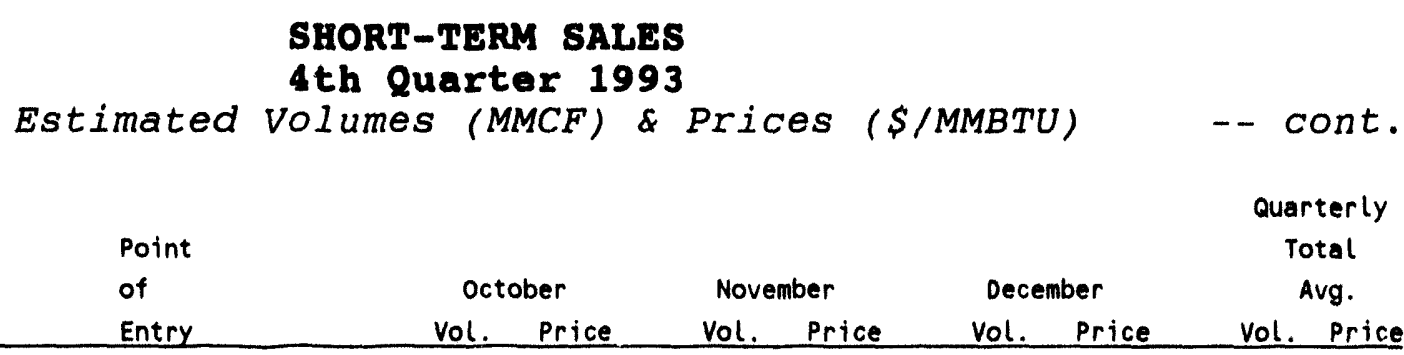

POCO PETROLEUM, INC.

Poco Petroleum Ltd.

Port land General Electric Company

Sumas, Washington $144 \quad 1.65$

$0 \quad$ N/A

O N/A

144

1.65

POCO PETROLEUM, INC.

Poco Petroleum Ltd.

Santanna Natural Gas

Noyes, Minnesota

$63 \quad 1.87$

O N/A

$0 \quad$ N/A

$63 \quad 1.87$

POCO PETROLEUM, INC.

Poco Petroleum Ltd.

Sierra Pacific Power Company

Eastport, Idaho

$7 \quad 1.83$

$17 \quad 1.76$

$0 \quad$ N/A

24

1.78

POCO PETROLEUM, INC.

Poco Petroleum Ltd.

Sierra Pacific Power Company

Sumas, Washington

$29 \quad 1.62$

$68 \quad 2.45$

$4 \quad 2.38$

102

2.21

POCO PETROLEUM, INC.

Poco Petroleum Ltd.

Sioux Pointe Inc.

Babb, Montana

$\begin{array}{ll}74 & 1.85\end{array}$

O N/A

$0 \quad$ N/A

$74 \quad 1.85$

POCO PETROLEUM, INC.

Poco Petroleum Ltd.

Sioux Pointe Inc.

Noyes, Minnesota

$112 \quad 1.80$

O N/A

O N/A

112

1.80

POCO PETROLEUM, INC.

Poco Petroleum Ltd.

Southwest Gas Corporation

Eastport, Idaho

O N/A

$3 \quad 1.75$

$10 \quad 1.90$

$13 \quad 1.87$

POCO PETROLEUM, INC.

Poco Petroleum Ltd.

Southwest Gas Corporation

Sumas, Washington

96

1.61

$0 \quad N / A$

10

2.28

106

1.67 


$$
\text { Page - } 125
$$

\section{SHORT-TERM SALES \\ 4th Quarter 1993}

Estimated Volumes (MMCF) \& Prices ( $\$ / M M B T U$ ) -- cont.

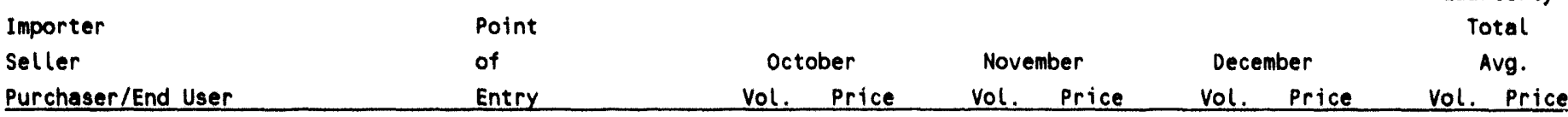

POCO PETROLEUM, INC.

Poco Petroleum Led.

Tristar Gas Marketing

Eastport, Idaho

$65 \quad 1.58$

$0 \quad$ N/A

$0 \quad N / A$

$65 \quad 1.58$

POCO PETROLEUM, INC.

Poco Petroleum Ltd.

Washington Natural Gas Company

Sumas, Washington

$0 \quad$ N/A

150

2.34

155

2.34

305

2.34

POCO PETROLEUM, INC.

Poco Petroleum Ltd.

Windward Energy \& Marketing inc.

Eastport, Idaho

$3 \quad 2.03$

D N/A

35

2.50

38

2.46

POCO PETROLEUM, INC.

Poco Petroleum Ltd.

Windward Energy \& Marketing Inc.

Sumas, Washington

$0 \quad$ N/A

O N/A

$3 \quad 2.65$

$3 \quad 2.65$

PORTLAND GENERAL ELECTRIC COMPANY

Unocal Canada Limited

Portland General Electric Company

$0 \quad$ N/A

$21 \quad 2.00$

$21 \quad 2.00$

PROGAS U.S.A., INC.

Progas Limited

AIG Trading

Noyes, Minnesota

$85 \quad 1.73$

O N/A

$0 \quad N / A$

$85 \quad 1.73$

PROGAS U.S.A., INC.

Progas Limited

CNG Gas Services

Niagara Falls, NY $152 \quad 2.06$

285

2.16

300

2.35

737

2.22

PROGAS U.S.A., INC.

Progas Limited

Cibola Corporation

Port of Morgan, MT 0 N/A

$0 \quad N / A$

464

2.12

464

2.12 


$$
\text { Page }-126
$$

\section{SHORT-TERM SALES}

4th Quarter 1993

Estimated Volumes (MMCF) \& Prices (\$/MMBTU) -- cont.

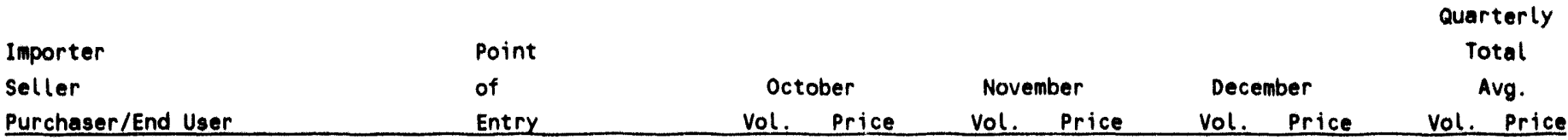

PROGAS U.S.A., INC.

Progas Limited

City of Perham, MN

Noyes, Minnesota

$43 \quad 1.73$

$47 \quad 1.88$

62

2.12

152

1.94

PROGAS U.S.A., INC.

Progas Limited

Coenergy Ventures

Noyes, Minneso

136

1.73

$419 \quad 1.88$

472

2.12

1027

1.97

PROGAS U.S.A., INC. Progas Limited

Equitable Resources

Niagara Falls, NY

$27 \quad 2.06$

$0 \quad N / A$

$0 \quad N / A$

$27 \quad 2.06$

PROGAS U.S.A., INC.

Progas Limited

Equitable Resources

Noyes, Minnesota

$0 \quad$ N/A

$0 \quad$ N/A

62

2.12

62

2.12

PROGAS U.S.A., INC.

Progas Limited

Great Plains Natural Gas Company

Noyes, Minnesota

229

1.73

$258 \quad 1.88$

310

2.12

797

1.93

PROGAS U.S.A., INC.

Progas Limited

Indeck Energy Services of Yerkes, I Noyes, Minnesota

$0 \quad N / A$

$0 \quad N / A$

$2 \quad 2.12$

2.12

PROGAS U.S.A., INC.

Progas Limited

Kaztex Energy Management

Noyes, Minnesota

$258 \quad 1.73$

$24 \quad 1.88$

94

2.12

377

1.84

PROGAS U.S.A., INC.

Progas Limited

Medina Power Company

Niagara Falls, NY $16 \quad 2.06$

$79 \quad 2.16$

N/A

95

2.14 


$$
\text { Page - } 127
$$

\section{SHORT-TERM SALES \\ 4th Quarter 1993}

Estimated Volumes (MMCF) \& Prices (S/MMBTU) - cont.

Quarterly

$\begin{array}{llll}\text { Importer } & \text { Point Total } \\ \text { Seller } & \text { of Avg. }\end{array}$

Purchaser/End User Entry vol. Price Vol. Price Vol. Price Vol. Price

PROGAS U.S.A., INC.

Progas Limited

Meridian Marketing

Niagara Falls, NY $0 \quad$ N/A

$3 \quad 2.16$

$0 \quad N / A$

32.16

PROGAS U.S.A., INC.

Procas Linited

Michigan Gas Ventures

Noyes, Minnesot

0 N/A

$275 \quad 1.88$

310

2.12

585

2.01

PROGAS U.S.A., INC.

Probas Linited

N.Y. State Electric \& Gas

Niagara Falls, NY 0 N/A

$86 \quad 2.16$

$97 \quad 2.35$

182

2.26

PROGAS U.S.A., INC.

Progas Linited

National fuel Resources

Niagara Falls, NY $105 \quad 2.06$

$0 \quad N / A$

$0 \quad$ N/A

$105 \quad 2.06$

PROGAS U.S.A., INC.

Prosas Limited

Natural Gas Clearinghouse

Niegara Falls, NY 0 N/A

O N/A

$92 \quad 2.35$

92

2.35

Progas U.S.A., INC.

Progas Limited

Natural Gas Clearinghouse

Port of Morgan, MT 0 N/A

0 N/A

155

2.12

155

2.12

Progas U.S.A., INC.

Procas Limited

O 8 R Energy

Niegara falls, NY $171 \quad 2.06$

$8 \quad 2.16$

o N/A

179

2.06

PRogas U.S.A., INC.

Proses Linited

Olympic Fuels, Inc.

Noyes, Minnesota $\quad 100 \quad 1.73$

$0 \quad N / A$

$0 \quad$ N/A

$100 \quad 1.73$ 


$$
\text { Page }-128
$$

\section{SHORT-TERM SALES}

4th Quarter 1993

Estimated Volumes (MMCF) \& Prices (\$/MMBTU) -- cont.

Importer

seller

Point

of October

Vol. Price

November

December

Quarterly

Total

Purchaser/End User

Entry

Vol. Price

Vol. Price

Avg.

Yol. Price

PROGAS U.S.A., INC.

Progas Limited

Olympic Fuels, Inc.

Port of Morgan, MT $0 \quad$ N/A

$0 \quad N / A$

$142 \quad 2.12$

142

2.12

PROGAS U.S.A., INC.

Progas Limited

Public Service Gas \& Electric

Niagara Falls, NY $\quad 15 \quad 2.06$

354

2.16

305

2.35

674

2.24

PROGAS U.S.A., INC.

ProGas Limited

Tenaska Marketing Ventures

Port of Morgan, MT 0 N/A

O N/A

232

2.12

232

2.12

PROGAS U.S.A., INC.

ProGas Limited

Western Gas Resources

Niagara Falls, NY $0 \quad$ N/A

$31 \quad 2.16$

$0 \quad N / A$

31

2.16

RENAISSANCE ENERGY (U.S.), INC.

Renaissance Energy Ltd.

C Energy, Inc.

Port of Morgan, Mr

$0 \quad N / A$

$150 \quad 1.59$

155

2.03

305

1.81

RENAISSANCE ENERGY (U.S.), INC.

Renaissance Energy Ltd.

Coastal Gas Marketing Company

Port of Morgan, MT $\quad 78 \quad 1.59$

$0 \quad N / A$

O N/A

78

1.59

RENAISSANCE ENERGY (U.S.), INC.

Renaissance Energy Ltd.

Connecticut Natural Gas

Waddington, NY

$0 \quad N / A$

206

2.28

O N/A

206

2.28

RENAISSANCE ENERGY (U.S.), INC.

Renaissance Energy Ltd.

Medina Power Company

Niagara Falls, NY $0 \quad$ N/A

$0 \quad N / A$

69

2.60

69

2.60 


$$
\text { Page - } 129
$$

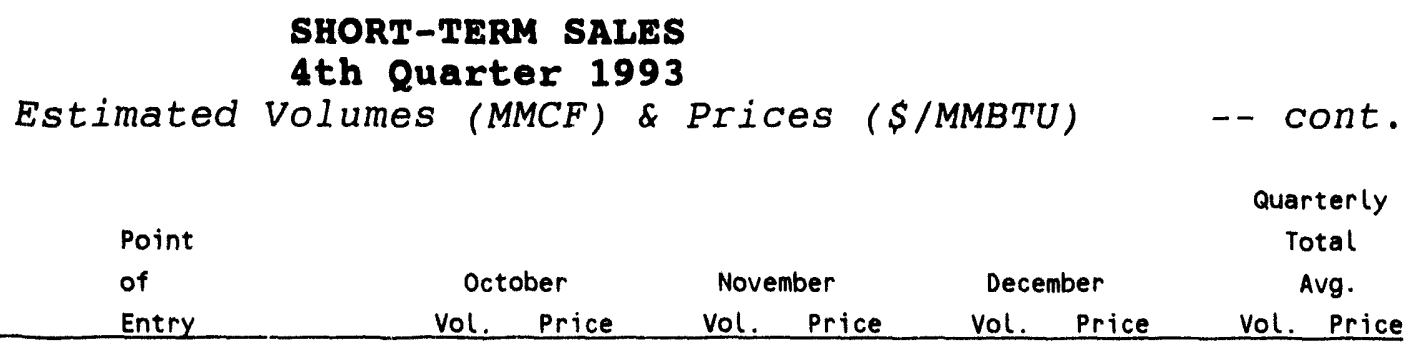

RENAISSANCE ENERGY (U.S.), INC.

Renaissance Energy Ltd.

Michigan Gas Company

Port of Morgan, MT

$63 \quad 1.59$

$0 \quad N / A$

$\begin{array}{ll}58 & 2.01\end{array}$

121

1.79

RENAISSANCE ENERGY (U.S.), INC. Renaissance Energy Ltd.

Minnegasco

RENAISSANCE ENERGY (U.S.), INC.

Renaissance Energy Ltd.

O \& R Energy

Waddington, NY

$0 \quad N / A$

$139 \quad 2.26$

o N/A

139

2.26

RENAISSANCE ENERGY (U.S.), INC. Renaissance Energy Ltd.

Peoples Natural Gas Company

Noyes, Minnesota $\quad 0 \quad \mathrm{~N} / \mathrm{A}$

O N/A

$61 \quad 2.32$

$61 \quad 2.32$

RENAISSANCE ENERGY (U.S.), INC. Renaissance Energy Ltd.

Peoples Natural Gas Company

Port of Morgan, MT $149 \quad 1.58$

$72 \quad 1.57$

$95 \quad 1.98$

$317 \quad 1.70$

RENAISSANCE ENERGY (U.3.), INC. Renaissance Energy Ltd.

phibro oil \& Gas Inc.

Niagara Falls, NY $0 \quad$ N/A

$89 \quad 2.29$

O N/A

$89 \quad 2.29$

RENAISSANCE ENERGY (U.S.), INC. Renaissance Energy Ltd.

Phoenix Chemical

Port of Morgan, M

158

1.58

O N/A

D

N/A

$158 \quad 1.58$

RENAISSANCE ENERGY (U.S.), INC. Renaissance Energy Ltd.

sioux pointe Inc. 


$$
\text { Page - } 130
$$

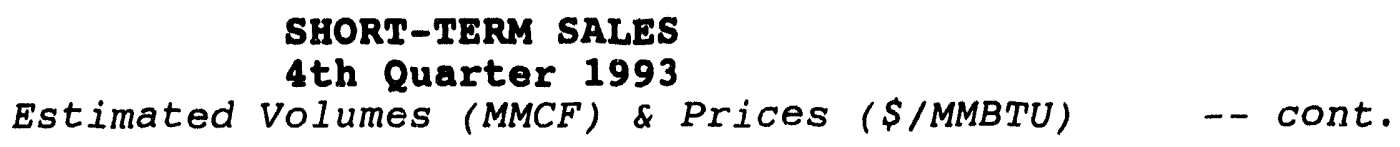

RENAISSANCE ENERGY (U.S.), INC.

Renaissance Energy Ltd.

Southern Connecticut Gas Company

Waddington, NY

$0 \quad$ N/A

O N/A

155

2.53

155

2.53

RENAISSANCE ENERGY (U.S.), INC.

Renaissance Energy Ltd.

Terra Industries

Port of Morgan, MT

O N/A

$75 \quad 1.56$

O N/A

$75 \quad 1.56$

RENAISSANCE ENERGY (U.S.), INC. Renaissance Energy Ltd.

Wisconsin Gas Port of Morgan, MT $93 \quad 1.58$

$0 \quad$ N/A

O N/A

$93 \quad 1.58$

RENAISSANCE ENERGY (U.S.), INC.

Renaissance Energy Ltd.

Wisconsin Power \& Light

Port of Morgan, MT $\quad 78 \quad 1.58$

$0 \quad$ N/A

$0 \quad$ N/A

$78 \quad 1.58$

SALMON RESOURCES LTD.

Shell Canada Limited

City of Ellensburg, WA

$$
\text { Sumas, Washington }
$$

$30 \quad 1.56$

88

2.13

$0 \quad N / A$

$118 \quad 1.99$

SALMON RESOURCES LTD.

Shell Canada Limited

Eastex Hydrocarbons

Waddington, NY

o N/A

$0 \quad N / A$

650

2.41

650

2.41

SALMON RESOURCES LTD. Shell Canada Linited

J. Maron Company

Sumas, Washington

$67 \quad 1.56$

0

N/A

$0 \quad N / A$

$67 \quad 1.56$

SALHON RESOURCES LTD. Shell Canada Limited 


\section{SHORT-TERM SALES}

4th Quarter 1993

Estimated Volumes (MMCF) \& Prices (\$/MMBTU) -- cont.

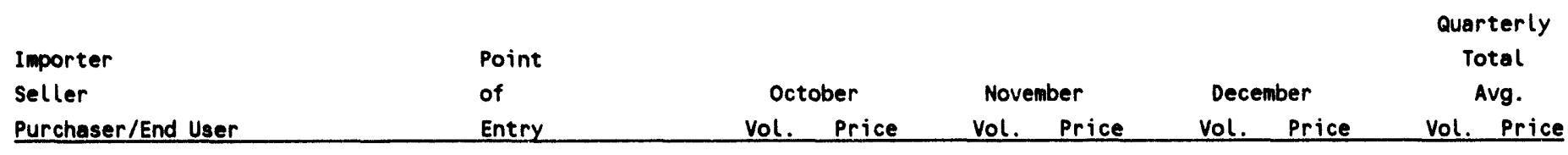

SALMON RESOURCES LTO.

Shell Canada Limited

Saimon Resources

Eastport, Idaho

O N/A

$940 \quad 1.53$

$934 \quad 1.76$

1874

1.64

SAN DIEGC GAS \& ELECTRIC

Sumit Resources Limited

San Diego Gas a Electric Company

Eastport, Idaho

$0 \quad N / A$

O N/A

$214 \quad 1.51$

214

1.51

SANTANMA MATURAL GAS CORPORATION Continental Energy Marketing Ltd. Brooklyn Interstate Matural Gas

$\begin{array}{lllllllll}\text { Haddington, NY } & 355 & 1.90 & 0 & \text { N/A } & 0 & \text { N/A } & 355 & 1.90\end{array}$

SIERRA PACIFIC PONER COMPANY Petro-Canada Hydrocarbons Inc. Sierra Pacific Pover Company Sumas, Hashington 4601.41 445

1.42

460

1.42

1365

1.42

SIERRA PACIFIC POUER COMPANY Shell Canada Limited

sierra Pacific Power Company

Susas, Washington

$459 \quad 1.47$

445

1.83

448

2.44

1353

1.91

SOUTHEASTERN MICHIGAN GAS COMPANY BP Western Gas Marketing Limited Southeastern Michigan Gas Company

SOUTHERN CALIFORNIA EDISON CONPANY American Hunter Exploration Ltd. Southern California Edison Campeny Eas vort, Idaho

O N/A

$48 \quad 2.13$

O N/A

48

2.13

SOUTHERA CALIFORNIA EDISON COMPANY Canedien Matural Resources Linited Southern California Edison Company 


$$
\text { Page - } 132
$$

\section{SHORT-TERM SALES \\ 4th Quarter 199.3}

Estimated Volumes (MMCF) \& Prices ( $\$ / M M B T U$ ) -- cont.

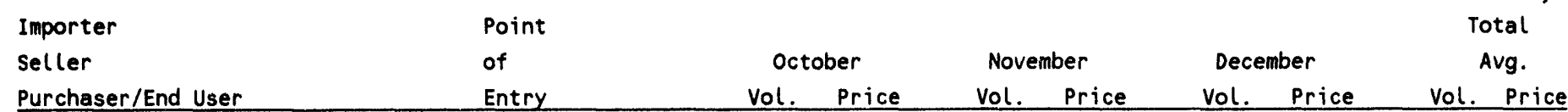

Entry

Vol. Price

Vol. Price

Vol. Price

Vol. Price

SOUTHERN CALIFORNIA EDISON COMPANY Cibola Canada

Southern California Edison Company

Eastport, Idaho

$0 \quad N / A$

351

1.96

191

2.00

542

1.97

SOUTHERN CALIFORNIA EDISON COMPANY Crestar Energy

Southern California Edison Company

Eastport, Idaho

O N/A

202

2.12

167

1.94

369

2.04

SOUTHERN CALIFORNIA EDISON COMPANY

Enron Gas Marketing Canada

Southern California Edison Company

Eastport, Idaho

$0 \quad N / A$

$0 \quad N / A$

129

1.92

129

1.92

SOUTHERN CALIFORNIA EDISON COMPANY

Fletcher Challenge Petroleum

Southern California Edison Company

Eastport, Idaho

$0 \quad N / A$

$58 \quad 1.87$

O N/A

$58 \quad 1.87$

SOUTHERN CALIFORNIA EDISON COMPANY

Home Oil Company Limited

Southern California Edison Company

Eastport, Idaho

$0 \quad N / A$

$0 \quad N / A$

$29 \quad 1.87$

$29 \quad 1.87$

SOUTHERN CALIFORNIA EDISON COMPANY

ICC Energy Corporation

Southern California Edison Company

Eastport, Idaho

$0 \quad N / A$

35

2.05

106

1.92

141

1.95

SOUTHERN CALIFORNIA EDISON COMPANY Northridge Gas Marketing

Southern California Edison Company

Eastport, Idaho

O N/A

36

1.96

12

1.90

165

1.91

SOUTHERN CALIFORNIA EDISON COMPANY Pan-Alberta Gas Ltd.

Southern California Edison Company
Eastport, Idaho
557

2.16
1.99

904

2.09 


$$
\text { Page - } 133
$$

\section{SHORT-TERM SALES}

4th Quarter 1993

Estimated Volumes (MMCF) \& Prices (\$/MMBTU) -- cont.

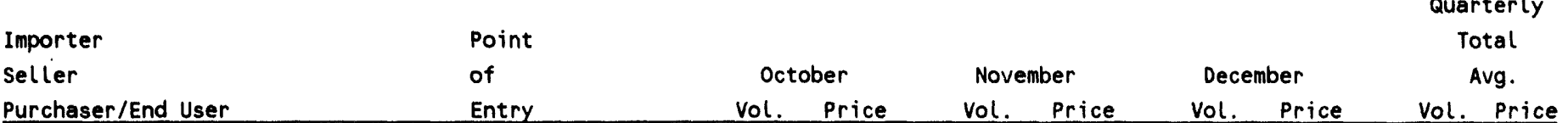

SOUTHERN CALIFORNIA EDISON COMPANY

ProGas Limited

Southern California Edison Company

Eastport, Idaho

$0 \quad$ N/A

$0 \quad$ N/A

149

1.90

149

1.90

SOUTHERN CALIFORNIA EDISON COMPANY Sceptre Resources Ltd.

Southern California Edison Company

Eastport, Idaho

$0 \quad N / A$

$48 \quad 2.25$

O N/A

48

2.25

SOUTHERN CALIFORNIA EDISON COMPANY USGT

Southern California Edison Company

Eastport, Idaho

$0 \quad$ N/A

O N/A

15

2.34

15

2.34

SOUTHERN CALIFORNIA EDISON COMPANY West Coast Gas Services Inc.

Southern California Edison Company

Eastport, Idaho

O N/A

79

2.51

0

N/A

$79 \quad 2.51$

SOUTHERN CALIFORNIA EDISON COMPANY Western Gas Marketing Limited Southern California Edison Company

Eastport, Idaho

O N/A

163

2.06

186

1.78

349

1.91

SOUTHERN CALIFORNIA EDISON COMPANY Western Gas Services

Southern California Edison Company

Eastport, Idaho

$0 \quad N / A$

$0 \quad N / A$

$147 \quad 1.97$

147

1.97

SOUTHWEST GAS CORPORATION

Mobil Oil Canada Ltd.

Southwest Gas Corporation

Sumas, Washington $146 \quad 1.65$

O N/A

O N/A

146

1.65

SUNCOR INC.

Suncor Inc.

Aquila Energy Marketing

Port of Morgan, MT $465 \quad 1.54$

O N/A

$0 \quad N / A$

465

1.54 


$$
\text { Page - } 134
$$

\section{SHORT-TERM SALES \\ 4th Quarter 1993}

Estimated Volumes (MMCF) \& Prices ( $\$ / M M B T U$ )

- cont.

Quarterly Total

Importer

Seller

Point

of

October

Vol. Price

November

Vol. Price

December

Vol. Price

Avg.

Vol. Price

SUNCOR INC.

Suncor Inc.

Bridgegas

Port of Morgan, MT

$73 \quad 1.54$

$170 \quad 1.59$

255

1.82

498

1.70

SUNCOR INC.

Suncor Inc.

City of Palo Alto, CA

Eastport, Idaho

$0 \quad$ N/A

$368 \quad 1.59$

$402 \quad 1.82$

770

1.71

SUNCOR INC.

Suncor Inc.

Coastal Gas Marketing Company

Port of Morgan, MT 0 N/A

$519 \quad 1.59$

387

1.82

906

1.69

SUNCOR INC.

Suncor Inc.

Enron Gas Marketing

Port of Morgan, MT $155 \quad 1.54$

$300 \quad 1.59$

$155 \quad 1.82$

610

1.64

SUNCOR INC.

suncor Inc.

Inland Steel Company

Port of Morgan, MT 0 N/A

$6 \quad 1.59$

127

1.82

$133 \quad 1.81$

SUNCOR INC.

Suncor Inc.

Louis Dreyfus Energy Corporation

Port of Morgan, MT $310 \quad 1.54$

$0 \quad N / A$

O N/A

310

1.54

SUNCOR INC.

Suncor Inc.

Metropolitan Utilities

Port of Morgan, MT 0 N/A

$92 \quad 1.59$

158

1.82

$250 \quad 1.74$

SUNCOR INC.

suncor Inc.

Midwest Gas

Port of Morgan, MT $474 \quad 1.54$

$0 \quad N / A$

115

1.82

590

1.59 


\section{SHORT-TERM SALES}

4th Quarter 1993

Estimated Volumes (MMCF) \& Prices (\$/MMBTU) -- cont. Quarterly Total

$\begin{array}{lll}\text { Importer } & \text { Point Total } \\ \text { Seller } & \text { of } & \text { October November }\end{array}$

Purchaser/End User Entry

Vol. Price

Vol. Price

Vol.

Vol. Price

SUNCOR INC.

Suncor Inc.

National Gas Resources

Port of Morgan, MT 0 N/A

$82 \quad 1.59$

$0 \quad$ N/A

$82 \quad 1.59$

SUNCOR INC.

Suncor Inc.

Peoples Natural Gas Company

Port of Morgan, MT $79 \quad 1.54$

$0 \quad N / A$

$0 \quad$ N/A

$79 \quad 1.54$

SUNCOR INC.

Suncor Inc.

Redwood Resources Inc.

Eastport, Idaho

O N/A

378

1.59

311

1.82

688

1.69

SUNCOR INC.

Suncor Inc.

San Diego Gas \& Electric Company

Eastport, Idaho

$0 \quad N / A$

$289 \quad 1.59$

$0 \quad$ N/A

289

1.59

SUNCOR INC.

Suncor Inc.

Sunrise Energy

Eastport, Idaho

$0 \quad N / A$

$0 \quad$ N/A

445

1.82

445

1.82

SUNCOR INC.

Suncor Inc.

Terra Industries

Port of Morgan, MT $158 \quad 1.54$

$153 \quad 1.59$

$158 \quad 1.82$

$469 \quad 1.65$

SUNCOR INC.

Suncor Inc.

Watsonville Cogeneration

Eastport, Idaho

$0 \quad N / A$

126

1.59

145

1.82

271

1.71

SUNCOR INC.

suncor Inc.

Wisconsin Power \& Light

Port of Morgan, MT 316

1.54

18

1.59

195

1.82

69

1.63 


$$
\text { Page - } 136
$$

\section{SHORT-TERM SALES \\ 4th Quarter 1993 \\ Estimated Volumes (MMCF) \& Prices (\$/MMBTU) -- cont.}

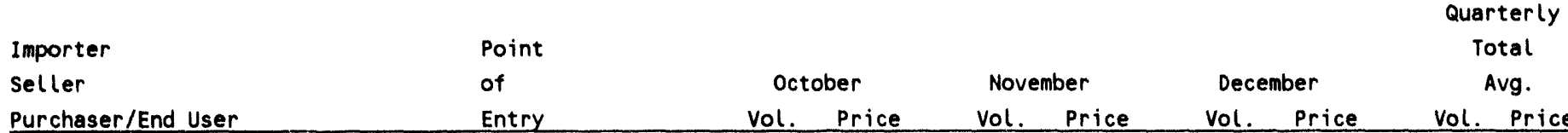

TARPON GAS MARKETING LTD.

Tarpon Gas Marketing Ltd.

Direct Gas

Waddington, NY

$0 \quad N / A$

56

2.29

$0 \quad N / A$

56

2.29

TARPON GAS MARKETING LTD.

Tarpon Gas Marketing Ltd.

O \& R Energy

Waddington, NY

$0 \quad N / A$

31

2.29

$0 \quad N / A$

31

2.29

TENNESSEE GAS PIPELINE COMPANY

Western Gas Services

Tennessee Gas Pipeline Company

Noyes, Minnesota

1653

1.70

O N/A

$0 \quad N / A$

1653

1.70

THE MONTANA PUWER COMPANY

Shell Canada Limited

The Montana Power Company

Babb, Montana

$0 \quad N / A$

$0 \quad N / A$

153

2.10

153

2.10

TRANSCO ENERGY MARKETING COMPANY

Canstates Gas Marketing

Baltimore Gas \& Electric

431

2.75

0

N/A

431

2.75

TRANSCO ENERGY MARKETING COMPANY

Canstates Gas Marketing

Canstates Gas Marketing

Niagara Falls, NY $11 \quad 2.18$

$23 \quad 2.50$

$0 \quad N / A$

34

2.40

TRANSCO ENERGY MARKETING COMPANY Canstates Gas Marketing

City of Fayetteville, NC

Niagara Falls, NY

$0 \quad N / A$

$0 \quad N / A$

23

1.94

23

1.94

TRANSCO ENERGY MARKETING COMPANY Canstates Gas Marketing

city of Greenwood, SC

Niagara Falls, NY $0 \quad \mathrm{~N} / \mathrm{A}$

D N/A

22

1.94

22

1.94 


$$
\text { Page - } 137
$$

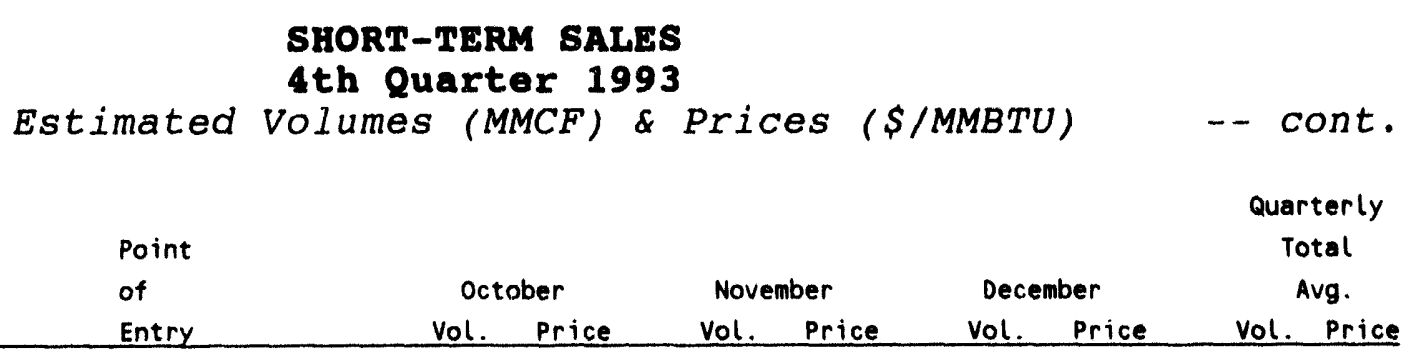

TRANSCO ENERGY MARKETING COMPANY

Canstates Gas Marketing

Clinton Gas

Niagara Falls, NY

O N/A

$178 \quad 1.84$

$0 \quad N / A$

$178 \quad 1.84$

TRANSCO ENERGY MARKETING COMPANY

Canstates Gas Marketing

Coastal Gas Marketing Company

Niagara falls, NY

24

2.15

$0 \quad$ N/A

O N/A

24

2.15

TRANSCO ENERGY MARKETING COMPANY Canstates Gas Marketing

Continental Energy Marketing L.td.

Niagara falls, NY $128 \quad 2.19$

53

1.98

127

1.93

307

2.05

TRANSCO ENERGY MARKETING COMPANY Canstates Gas Marketing

Direct Energy

Niagara Falls, NY

124

2.19

103

2.30

O N/A

227

2.24

TRANSCO ENERGY MARKETING COMPANY Canstates Gas Marketing

Hazelton Fuel Management Company

Niagara Falls, NY

$0 \quad$ N/A

296

2.63

639

2.63

935

2.63

TRANSCO ENERGY MARKETING COMPANY Canstates Gas Marketing Hopewell Cogeneration Facility Niagara Falls, NY $\quad 31 \quad 1.88$

$29 \quad 2.00$

101

1.88

161

1.90

TRANSCO ENERGY MARKETING COMPANY Canstates Gas Marketing Hub Services

Niagara Falls, NY

$$
0 \quad N / A
$$

$3 \quad 1.84$

O N/A

$3 \quad 1.84$

TRANSCO ENERGY MARKETING COMPANY Canstates Gas Marketing KCS Energy Management

Niagara Falls, NY $\quad 74 \quad 2.25$
0 N/A

$0 \quad$ N/A

74

2.25 


$$
\text { Page - } 138
$$

\section{SHORT-TERM SALES \\ 4th Quarter 1993}

Estimated Volumes (MMCF) \& Prices (\$/MMBTU) -- cont.

Importer

seller

Point

of

October

November

Quarterly

Total

Purchaser/End User

Entry

Vol. Price

Vol. Price

December

Avg.

Vol. Price

Vol. Price

TRANSCO ENERGY MARKETING COMPANY

Canstates Gas Marketing

Long Is land Lighting Company

Niagara Falls, NY $678 \quad 1.20$

O N/A

O N/A

678

1.20

TRANSCO ENERGY MARKETING COMPANY Canstates Gas Marketing

National Fuel Gas Distribution

Niagara Falls, NY

0 N/A

$29 \quad 1.55$

$0 \quad N / A$

$29 \quad 1.55$

TRANSCO ENERGY MARKETING COMPANY

Canstates Gas Marketing

National fuel Resources

Niagara Falls, NY

0 N/A

$97 \quad 1.58$

$0 \quad$ N/A

$97 \quad 1.58$

TRANSCO ENERGY MARKETING COMPANY

Canstates Gas Marketing

Philadelphia Electric Company

Niagara Falls, NY $0 \quad$ N/A

O N/A

48

2.83

48

2.83

TRANSCO ENERGY MARKETING COMPANY

Canstates Gas Marketing

Public Service Gas \& Electric

Niagara Falls, NY $54 \quad 1.12$

O N/A

$0 \quad$ N/A

54

1.12

TRANSCO ENERGY MARKETING COMPANY Canstates Gas Marketing

Public Service of North Carolina

Niagara falls, NY $0 \quad$ N/A

$164 \quad 1.94$

322

1.38

486

1.57

TRANSCO ENERGY MARKETING COMPANY Canstates Gas Marketing

Union Gas Company

Niagara falls, NY 0 N/A

TRANSCO ENERGY MARKETING COMPANY Cont inental Energy Marketing Ltd. Consumers' Gas Company Ltd.
Niagara Falls, NY $0 \quad$ N/A
164

2.87
164

2.87 


$$
\text { Page - } 139
$$

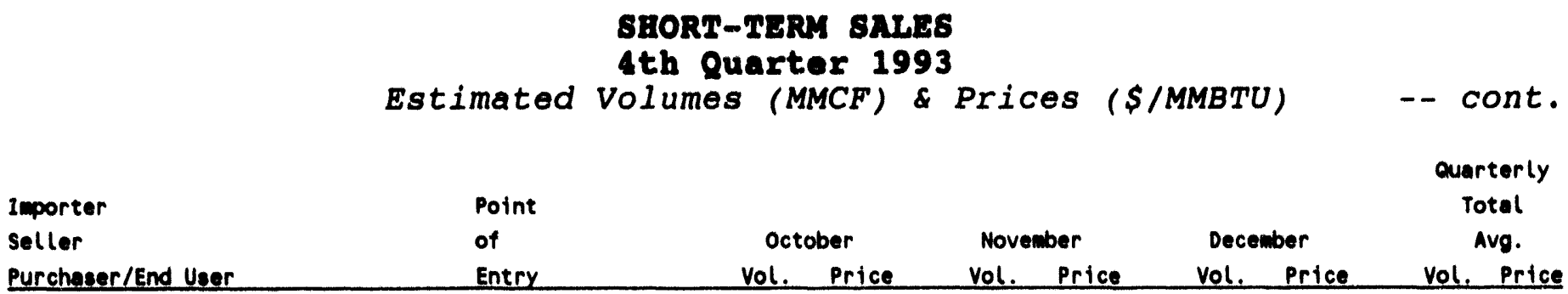

TRANSCO ENERGY MARKETING COMPANY Direct Energy Marketing Limited Public Service of North Carolina

TRISTAR GAS MARKETING COMPANY Alberta Soutliern Company Ltd. Pacific Gas \& Electric Company

UNIVERSAL RESOURCES CORPORATION Mobil Oil Canada Ltd.

IGI Resources

VALERO INDUSTRIAL GAS, L.P.

P.M. I. Comercio Internacional Valero Industrial Gas

VECTOR ENERGY (U.S.A.) INC.

Vector Energy Inc.

Altresco Pittsfield, L.P.

VECTOR ENERGY (U.S.A.) INC.

Vector Energy Inc.

Pacific Gas \& Electric Company

VERMONT GAS SYSTEMS, INC.

Gaz Metropolitan, Inc.

Vermont Gas Systems, Inc.

VERMONT GAS SYSTEMS, INC.

Western Gas Marketing Limited

Vermont Gas Systems, Inc.
Niagara Falls, NY 0 N/A

29

2.88

$0 \quad$ N/A

29

2.88

Eastport, Idaho $\quad 568 \quad 1.49$

O N/A

D N/A

568

1.49

Sumas, Washington $\quad 179 \quad 1.75$

O N/A

O N/A

179

1.75

Hidalgo, Texas

$0 \quad N / A$

O N/A

133

1.88

133

1.88

Niagara falls, NY

629

2.99

$0 \quad N / A$

$0 \quad N / A$

629

2.99

Eastport, Idaho

O N/A

504

1.44

509

1.54

1013

1.49

Highgate Spr., VT

$0 \quad$ N/A

$0 \quad$ N/A

$3 * t * t$

3

Highgate Spr., VT $\quad 0 \quad$ N/A

$0 \quad N / A$

$49 \quad 2.67$

49

2.67 


$$
\text { Page - } 140
$$

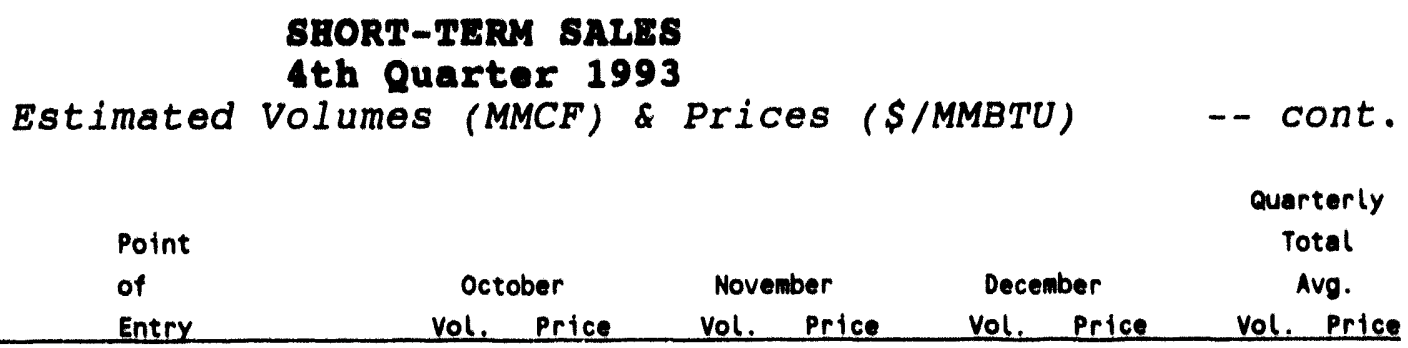

WASHINGTON ENERGY MARKETING, INC.

Ocelot Energy, Inc.

Boeing Company

Sumas, Washington

$155 \quad 1.54$

149

1.54

155

1.54

459

1.54

WASHINGTON ENERGY MARKETING, INC.

Talisman

Washington Energy

Sumas, Washington

$0 \quad$ N/A

$45 \quad 1.86$

$0 \quad$ N/A

$45 \quad 1.86$

WASHINGTON ENERGY MARKETING, INC. Washington Energy Marketing

Enron Gas Marketing

Eastport, Idaho

$0 \quad$ N/A

$667 \quad 1.80$

$0 \quad N / A$

667

1.80

WASHINGTON ENERGY MARKETING, INC.

Washington Energy Marketing

National Gas Resources

Eastport, Idaho

O N/A

20

2.13

$0 \quad N / A$

20

2.13

WASHINGTON ENERGY MARKETING, INC.

Washington Energy Marketing

sterra Pacific Power Company

Eastport, Idaho

O N/A

O N/A

$15 \quad 1.77$

$15 \quad 1.77$

WASHINGTON ENERGY MARKETING, INC. Westcoast Energy Marketing, Ltd.

Aluminum Company of America

Eastport, Idaho

O N/A

$75 \quad 1.97$

78

2.21

153

2.09

WASHINGTON ENERGY MARKETING, INC. Westcoast Energy Marketing, Ltd. Cascade Natural Gas

Eastport, Idaho

$0 \quad$ N/A

83

2.25

31

2.02

114

2.19

WASHINGTON ENERGY MARKETING, INC. Westcoast Energy Marketing, Ltd. chevron U.S.A. Inc. 


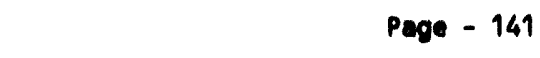

ATTACHMENT C-4

\section{SHORT-TERM BALES \\ Sth Quarter 1993}

Estimated Volumes (MMCF) \& Prices (\$/MMBTU) -- cont.

Importer Point

seller of

of October November

Quarterly

Total

Purchager/End User

Entry

Vol. Price

Vol. Price

December

Avg.

Vol. Price

Vol. Price

WASHINGTON ENERGY MARKETING, INC.

Westconst Energy Marketing, Ltd.

city of Buckley, OR

Eastport, Idaho

O N/A

$15 \quad 2.50$

13

2.02

$28 \quad 2.27$

WASHINGTON ENERGY MARKETING, INC. Westcosat Energy Marketing, Ltd.

city of Enumclaw, WA

Eastport, Idaho

O N/A

$39 \quad 2.50$

11

2.02

50

2.39

WASHINGTON ENERGY MARKETING, INC. Westcoast Energy Marketing, Ltd.

Encogen Nor thwest, L.P.

Eastport, Idaho

$0 \quad$ N/A

$14 \quad 1.78$

$59 \quad 1.30$

$73 \quad 1.39$

WASHINGTON ENERGY MARKETING, INC. Westcoast Energy Marketing, Ltd. Enron Gas Marketing

Eastport, Idaho

$0 \quad$ N/A

$0 \quad N / A$

$737 \quad 1.83$

$737 \quad 1.83$

WASHINGTON ENERGY MARKETING, INC. Westcoast Energy Market ing, Ltd. Grand Valley Gas Company

WASHINGTON ENERGY MARKETING, INC. Westcoost Energy Marketing, Ltd. IGI Resources

WASHINGTON ENERGY MARKETING, INC. West coast Energy Marketing, Ltd.

WASHINGTON ENERGY MARKETING, INC. Hestcosst Energy Marketing, Ltd. J.R. Simplot Company 


$$
\text { Page - } 142
$$

\section{SBORT-TERM SALES \\ 4th Quarter 1993}

Estimated Volumes (MMCF) \& Prices (\$/MMBTU)

- cont.

Importer

seller

Purchaser/End User

\section{Point}

of

Entry october

Vol. Price
Eastport, Idaho

$0 \quad N / A$

$0 \quad$ N/A

183

2.02

183

2.02

WASHINGTON ENERGY MARKETING, INC. Westcoast Energy Marketing, Ltd.

Portland General Electric Company
$0 \quad N / A$

Eastport, Idaho
O N/A

Westcoast Energy Marketing, Ltd.

WASHINGTON NATURAL GAS COMPANY Cantest Gas Supply inc.

Washington Natural Gas Company

WASHINGTON MATURAL GAS COMPANY

Canadian Hydrocarbons Marketing

Washington Natural Gas Company

Sumas, Washington

1.65

$0 \quad N / A$

Sumas, Washington $\quad 70 \quad 1.58$

$0 \quad$ N/A
O N/A

86

1.65

$0 \quad$ N/A

70

1.58

WASHINGTON MATURAL GAS COMPANY

Talisman

Washington Natural Gas Company

Sumas, Washington

0

N/A

200

2.26

240

2.38

440

2.33

WES CANA ENERGY MARKETING (U.S.) INC.

KannGaz Producers

Altresco Pittsfield, L.P.

Niagara Falls, NY $110 \quad 2.00$

$0 \quad$ N/A

$0 \quad N / A$

110

2.00

WES CANA ENERGY MARKETING (U.S.) INC.

KannGaz Producers

Direct Energy

Niagara falls, NY

144

2.00

142

2.14

352

2.00

637

2.03 


$$
\text { Page - } 143
$$

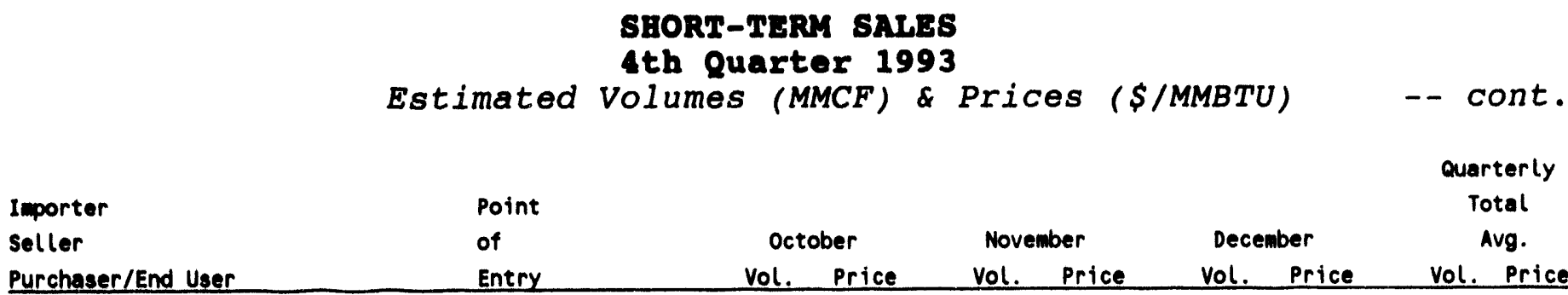

WES CANA ENERGY MARKETING (U.S.) INC.

KannGaz Producers

Eastex Hydrocarbons
Niagara Falls, NY $\quad 40 \quad 2.00$

$0 \quad N / A$

O N/A

40

2.00

WES CANA ENERGY MARKETING (U.S.) INC. KannGaz Producers

Enron Gas Marketing

Niagara Falls, NY

21

2.14

$0 \quad$ N/A

21

2.14
O N/A

WES CANA ENERGY MARKETING (U.S.) INC.

KannGaz Producers

Louis Dreyfus Energy Corporation

Niagara Falls, NY

$512 \quad 2.00$

$0 \quad$ N/A

(1)

$0 \quad N / A$

512

2.00

WES CANA ENERGY MARKETING (U.S.) INC.

KannGaz Producers

Meridian Marketing

Niagara Falls, NY

$0 \quad N / A$

104

2.14

152

2.00

256

2.06

WES CANA ENERGY MARKETING (U.S.) INC.

KannGaz Producers

National Fuel Gas Distribution

Niagara Falls, NY $0 \quad$ N/A

290

2.14

$0 \quad$ N/A

290

2.14

WES CANA ENERGY MARKETING (U.S.) INC.

KannGaz Producers

National Fuel Resources

Niagara falls, NY

290

2.00

146

2.14

$0 \quad$ N/A

436

2.05

WES CANA ENERGY MARKETING (U.S.) INC.

KannGaz Producers

New England Power Company

Niagara Falls, NY

O N/A

O N/A

363

2.00

363

2.00

WES CANA ENERGY MARKETING (U.S.) INC.

KannGaz Producers

Orange \& Rockland Utilities

Niagara Falls, NY $771 \quad 2.00$

747

2.14

771

2.00

2289

2.05 


$$
\text { Page - } 144
$$

\section{SHORT-TERM SALES \\ 4th Quarter 1993}

Estimated Volumes (MMCF) \& Prices (S/MMBTU) -- cont.

seller

of

October

November

Quarterly

Total

Purchaser/End User

Entry

Vol. Price

Vol. Price

December

Avg.

Vol. Price

WES CANA ENERGY MARKETING (U.S.) INC.

KannGaz Producers

Phibro 0il \& Gas Inc.

Niagara falls, NY 0 N/A

358

2.14

152

2.00

510

2.10

WES CANA ENERGY MARKETING (U.S.) INC.

KannGaz Producers

Prior Energy Corporation

Niegara falls, NY $61 \quad 2.00$

$0 \quad N / A$

$0 \quad N / A$

$61 \quad 2.00$

WES CANA ENERGY MARKETING (U.S.) -NC.

KannGaz Producers

Sunrise Energy

Niagara Falls, NY 32.00

$0 \quad N / A$

$0 \quad$ N/A

32.00

WES CANA ENERGY MARKETING (U.S.) INC.

Wes Cana Energy Marketing Inc.

Aquila Energy Marketing

Port of Morgan, MT $30 \quad 1.37$

$0 \quad$ N/A

$0 \quad N / A$

$30 \quad 1.37$

WES CANA ENERGY MARKETING (U.S.) INC.

Wes Cana Energy Marketing Inc.

Bridgegas

Port of Morgan, MT $10 \quad 1.37$

$0 \quad$ N/A

$19 \quad 1.39$

29

1.38

WES CANA ENERGY MARKETING (U.S.) INC.

Wes Cana Energy Marketing Inc.

Broad Street oil \& Gas

Port of Morgan, MT $155 \quad 1.37$

D N/A

$0 \quad N / A$

155

1.37

WES CANA ENERGY MARKETING (U.S.) INC.

Wes Cana Energy Marketing Inc.

Cibola corporation

Port of Morgan,

$268 \quad 1.37$

$141 \quad 1.66$

91

1.39

500

1.46

WES CANA ENERGY MARKETING (U.S.) INC.

Wes Cana Energy Marketing Inc.

Eastex Hydrocarbons

Port of Morgan,

$0 \quad$ N/A

151

1.66

$0 \quad N / A$

151

1.66 


$$
\text { Page - } 145
$$

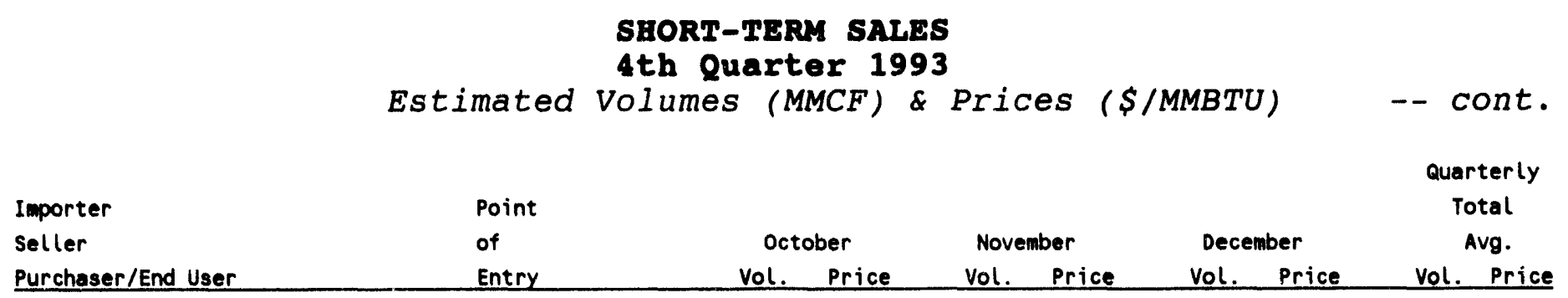

WES CANA ENERGY MARKETING (U.S.) INC.

Wes Cana Energy Marketing Inc.

Enron Gas Marketing

Port of Morgan, MT $0 \quad$ N/A

$0 \quad N / A$

$5 \quad 1.39$

5

1.39

WES CANA ENERGY MARKETING (U.S.) INC.

Wes Cana Energy Marketing Inc.

Northern Natural Gas

Port of Morgan, MT $0 \quad$ N/A

O N/A

$10 \quad 1.39$

$10 \quad 1.39$

WES CANA ENERGY MARKETIHG (U.S.) INC.

Wes Cana Energy Marketing Inc.

Olympic Fuels, Inc.

Port of Morgan, MT $\quad 155 \quad 1.37$

$151 \quad 1.66$

$0 \quad N / A$

306

1.51

WES CANA ENERGY MARKETING (U.S.) INC.

Wes Cana Energy Marketing Inc.

Prairieland Energy Marketing

Port of Morgan, MT $11 \quad 1.37$

O N/A

$0 \quad$ N/A

$11 \quad 1.37$

WES CANA ENERGY MARKETING (U.S.) INC.

Wes Cana Energy Marketing Inc.

Sioux Pointe Inc.

Port of Morgan, MiT $310 \quad 1.37$

$151 \quad 1.66$

$168 \quad 1.39$

628

1.45

WES CANA ENERGY MARKETING (U.S.) INC.

Wes Cana Energy Marketing Inc.

Tenaska Marketing Ventures

Port of Morgan, MT $27 \quad 1.37$

141

1.66

123

1.39

291

1.52

WES CANA ENERGY MARKETING (U.S.) INC.

Wes Cana Energy Marketing Inc.

Tristar Gas Marketing

Port of Morgan, MT $\quad 95 \quad 1.37$

$5 \quad 1.66$

69

1.39

169

1.39

WESTCOAST GAS STRVI' ¿S INC.

Unigas Corporatici:

cibola corporation

Noyes, Minnesota

$0 \quad N / A$

$8 \quad 1.88$

D N/A

$8 \quad 1.88$ 


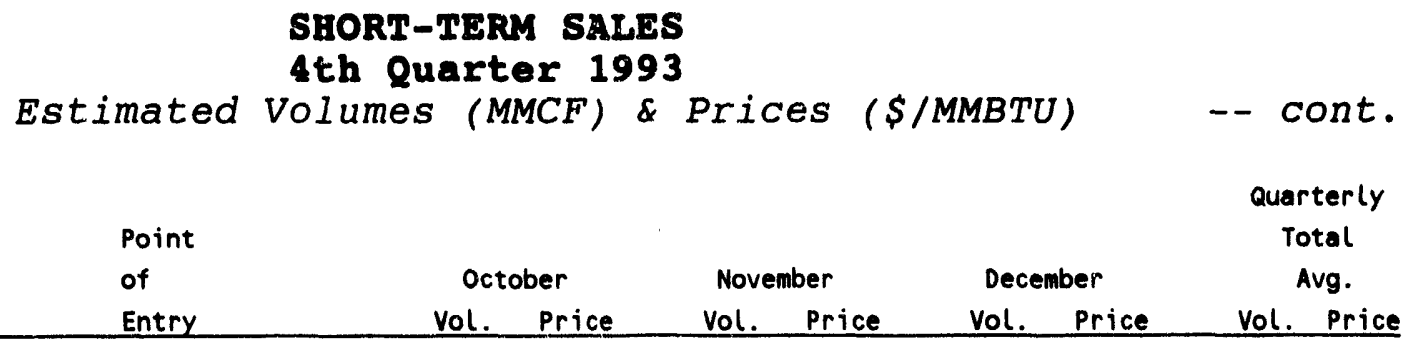

WESTCOAST GAS SERVICES INC.

Unigas Corporation

Cibola Corporation

Port of Morgan, MT 0 N/A

$12 \quad 2.04$

$0 \quad$ N/A

12

2.04

WESTCOAST GAS SERVICES INC.

Unigas Corporation

Industrial Energy Applications

Port of Morgan, MT 0 N/A

O N/A

214

2.05

214

2.05

WESTCOAST GAS SERVICES INC.

Unigas corporation

Interstate Power Company

Port of Morgan, MT $\quad 80 \quad 1.54$

$0 \quad$ N/A

$0 \quad N / A$

$80 \quad 1.54$

WESTCOAST GAS SERVICES INC.

Unigas Corporation

Midwest Gas

Port of Morgan, MT $77 \quad 1.55$

$90 \quad 1.58$

$4 \quad 1.61$

$172 \quad 1.57$

WESTCOAST GAS SERVICES INC.

Unigas Corporation

Minnegasco

Noyes, Minnesota 20

$0 \quad N / A$

$0 \quad N / A$

20

1.74

WESTCOAST GAS SERVICES INC.

Unigas Corporation

North Canadian Marketing Corp.

Port of Morgan, MT 15

1.59

$300 \quad 1.56$

$303 \quad 1.92$

761

1.71

WESTCOAST GAS SERVICES INC.

Unigas Corporation

Pancanadian Petroleum Ltd.

Port of Morgan, MT $410 \quad 1.55$

$0 \quad N / A$

$0 \quad$ N/A

410

1.55

WESTCOAST GAS SERVICES INC.

Unigas Corporation

Peoples Natural Gas Company

Noyes, Minnesota $\quad 158 \quad 1.77$

0 N/A

o N/A

158

1.77 


$$
\text { Page - } 147
$$

\section{SHORT-TERM SALES \\ 4th Quarter 1993}

Estimated Volumes (MMCF) \& Prices (\$/MMBTU) -- cont.

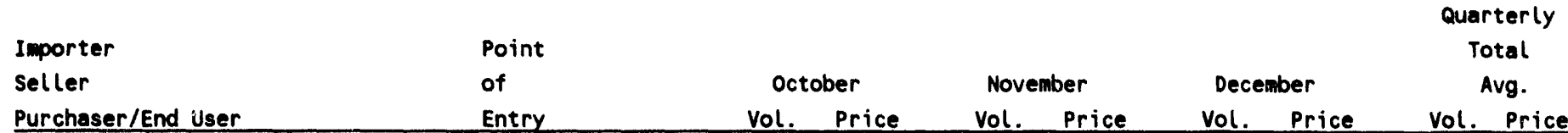

WESTCOAST GAS SERVICES INC.

Unigas Corporation

Peoples Natural Gas Company

Port of Morgan, MT

$223 \quad 1.54$

O N/A

O N/A

$223 \quad 1.54$

WESTCOAST GAS SERVICES INC.

Unigas Corporation

Potters Industries

Massena, New York $19 \quad 1.64$

$23 \quad 2.57$

12

2.57

$54 \quad 2.24$

WESTCOAST GAS SERVICES INC. Unigas Corporation

St. Lawrence University

Massena, New York

$12 \quad 1.63$

12

2.57

23

2.57

$47 \quad 2.33$

WESTCOAST GAS SERVICES INC. Unigas Corporation

Stone Container Corporation

Babb, Montana

$155 \quad 1.03$

O N/A

$0 \quad$ N/A

155

1.03

WESTCOAST GAS SERVICES INC. Unigas Corporation Unigas Noyes, Minnesota $140 \quad 1.81$

$0 \quad N / A$

$0 \quad$ N/A

$140 \quad 1.81$

WESTCOAST GAS SERVICES INC. Unigas Corporation Unigas Port of Morgan, MT $10 \quad 1.29$

178

2.14

N/A

188

2.10

WESTCOAST GAS SERVICES INC. West Coast Gas Services Inc. Unigas

WESTERN GAS MARKETING INC. Western Gas Marketing Limited Appalachian Gas Sales 


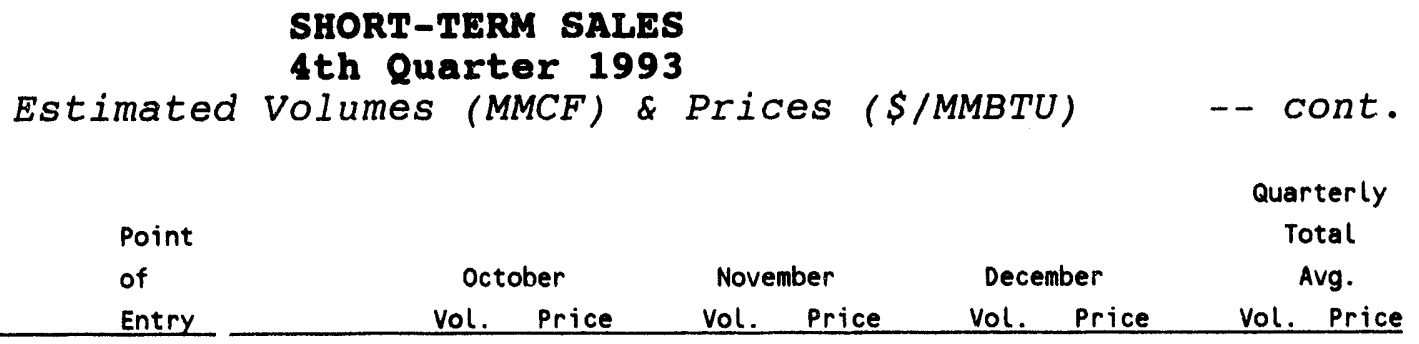

Importer

Seller

Purchaser/End User
Entry

Vol Price

$138 \quad 1.80$

$0 \quad$ N/A

138

1.80

Arco Products Company

WESTERN GAS MARKETING INC.

Western Gas Marketing Limited

Arkla Gas Marketing

Port of Morgan, MT $288 \quad 1.61$

$80 \quad 1.80$

$0 \quad$ N/A

$368 \quad 1.65$

WESTERN GAS MARKETING INC.

Western Gas Marketing Limited

Bearpaw

Babb, Montana

$0 \quad$ N/A

$12 \quad 1.50$

$0 \quad$ N/A

12

1.50

WESTERN GAS MARKETING INC. Western Gas Marketing Limited CNG Gas Services

Waddington, NY

$0 \quad$ N/A

590

2.27

447

2.44

1037

2.34

WESTERN GAS MARKETING INC. Western Gas Marketing Limited cibola Corporation

$\begin{array}{lllllllll}\text { Port of Morgan, MT } & 262 & 1.61 & 1026 & 1.80 & 921 & 1.75 & 2208 & 1.76\end{array}$

WESTERN GAS MARKETING INC.

Western Gas Marketing Limited clinton Gas

Waddington, NY

$70 \quad 2.09$

$47 \quad 2.27$

44

2.44

162

2.24

WESTERN GAS MARKETING INC. Western Gas Marketing Limited Coastal Gas Marketing Company

Port of Morgan, MT $343 \quad 1.61$

$472 \quad 1.80$

195

1.75

1010

1.73

WESTERN GAS MARKETING INC.

Western Gas Marketing Limited Coastal Gas Marketing Company

Waddington, NY
$0 \quad N / A$

7

2.44

7

2.44

l N/A

$0 \quad N / A$ 


$$
\text { Page - } 149
$$

SHORT-TERM SALES
4th Quarter 1993
Estimated Volumes (MMCF) \& Prices $(S / M M B T U)$

WESTERN GAS MARKETING INC.

Western Gas Marketing Limited

Continental Energy Marketing Ltd.

Waddington, NY

$176 \quad 2.09$

170

2.27

153

2.44

499

2.26

WESTERN GAS MARKETING INC. Western Gas Marketing Limited DGS Trading

WESTERN GAS MARKETING INC. Western Gas Marketing Limited DGS Trading

WESTERN GAS MARKETING INC. Western Gas Marketing Limited Development Associates

WESTERN GAS MARKETING INC. Western Gas Marketing Limited Entrade Corporation

WESTERN GAS MARKETING INC. Western Gas Marketing Limited Gaslantic

WESTERN GAS MARKETING INC. Western Gas Marketing Limited Goetz Energy Corporation

WESTERN GAS MARKETING INC. Western Gas Marketing Limited Hadson Gas Systems 


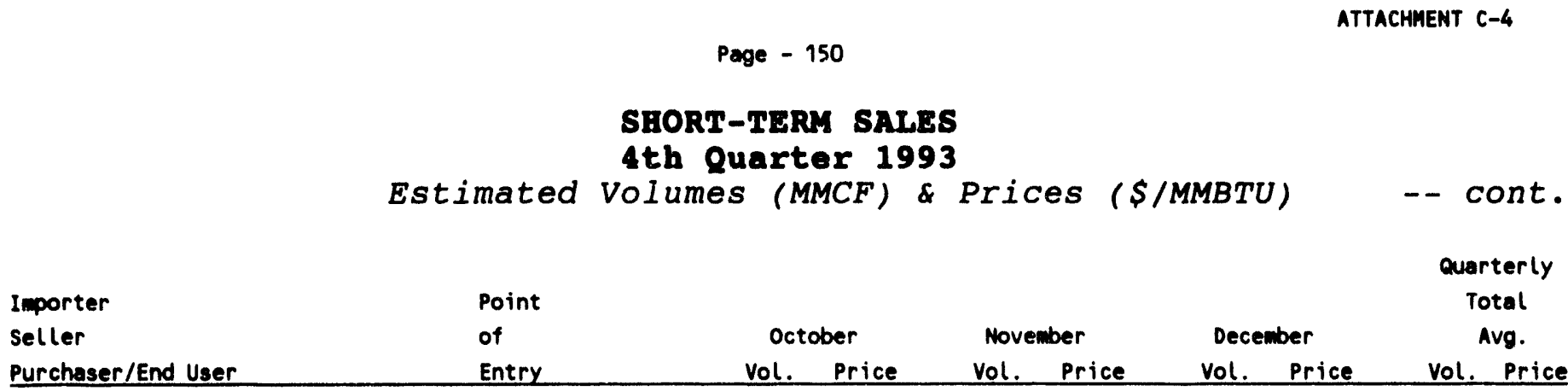

WESTERN GAS MARKETING INC.

Western Gas Marketing Limited

Hesse Gas Company

Noyes, Minnesota $\quad 321 \quad 1.74$

O N/A

$0 \quad$ N/A

321

1.74

WESTERN GAS MARKETING INC. Hestern Gas Marketing Limited Hesse Gas Company

Port of Morgan, MT 11

$673 \quad 1.75$

1308

1.77

WESTERN GAS MARKETING INC.

Western Gas Marketing Limited Hub Services

Niagara Falls, NY 0

$0 \quad N / A$

183

2.54

183

2.54

WESTERN GAS MARKETING INC.

Western Gas Marketing Limited ICC

$0 \quad N / A$

$17 \quad 1.75$

80

1.64

WESTERN GAS MARKETING INC.

Western Gas Marketing Limited

IGI Resources

Eastport, Idaho

$0 \quad N / A$

$504 \quad 1.85$

O N/A

$504 \quad 1.85$

WESTERN GAS MARKETING INC.

Western Gas Marketing Limited Interenergy Corporation

Babb, Montana

O N/A

$9 \quad 1.50$

16

2.44

25

2.10

WESTERN GAS MARKETING INC. Western Gas Marketing Limited Kaztex Energy Management

Port of Morgan, MT 18

$21 \quad 1.80$

129

1.75

$168 \quad 1.74$

WESTERN GAS MARKETING INC. Western Gas Marketing Limited Kimball Trippe Inc.

$13 \quad 2.05$
1.77

(1)




$$
\text { Page - } 151
$$

\section{SHORT-TERM SALES \\ 4th Quarter 1993}

Estimated volumes (MMCF) \& Prices (\$/MMBTU) -- cont.

$\begin{array}{lllll}\text { Inporter } & \text { Point } & & \text { Quarterly } \\ \text { Seller } & \text { of } & & & \text { Total } \\ \text { Purcheser/End User } & \text { Entry } & \text { October } & \text { November } & \text { December } \\ \text { Avg. }\end{array}$

WESTERN GAS MARKETIMG INC.

Western Gas Marketing Limited

Michigan Gas Company

Port of Morgan, MT $138 \quad 1.61$

$253 \quad 1.80$

$215 \quad 1.75$

$605 \quad 1.74$

WESTERN GAS MARKETING INC.

Western cas Marketing Limited

Midland cogeneration Venture

Noyes, Minnesota $117 \quad 1.77$

o N/A

O N/A

$117 \quad 1.77$

WESTER GAS MARKETING INC.

Western Gas Marketing Limited Midland Market ing

$\begin{array}{lllllllll}\text { Port of Morgan, MT } & 0 & \text { N/A } & 152 & 1.80 & 135 & 1.75 & 287 & 1.78\end{array}$

UESTERA GAS MARKETING INC. Western Gas Marketing Linited Matural Gas Clearinghouse Port of Morgan, HT $47 \quad 1.61$

$0 \quad \mathbf{N} / \mathbf{A}$

$0 \quad N / A$

$47 \quad 1.61$

WESTERN GAS MARKETING INC.

Western Gas Marketing Linited Matural Gas Clearinghouse

Waddington, NY

- N/A

O N/A

$5 \quad 2.44$

$5 \quad 2.44$

WESTERN GAS MARKETIMG IMC. Western Gas Marketing Limited Neste oy

Eastport, Idaho

$0 \quad N / A$

$0 \quad N / A$

1025

1.80

1025

1.80

WESTERM GAS MARETIMG IMC.

Western Gas Marketing Limited Meate oy

Port of Horgan, MT $0 \quad$ N/A

$0 \quad N / A$

313

1.75

313

1.75

WESTERW GAS MARKeTING IMC. Western Gas Marketing Limited Nipsco Energy Treding Corporation Port of norgan, nT $162 \quad 1.61$ 152 1.80

O N/A 


$$
\text { Page - } 152
$$

\section{SHORT-TERM SALES \\ 4th Quarter 1993 \\ Estimated Volumes (MMCF) \& Prices (\$/MMBTU) -- cont.}

Importer

Seller

Purchaser/End User
Point

of

Entry
Port of Morgan, MT $68 \quad 1.61$

Western Gas Marketing Limited

North Canadien Marketing Corp.

WESTERN GAS MARKETING INC.

Western Gas Marketing Limited

Northern Illinois Gas

WESTERN GAS MARKETING INC.

Western Gas Marketing Limited

Northern States Power (MN)

WESTERN GAS MARKETING INC.

Western Gas Marketing Limited

Northern States Power Company

WESTERN GAS MARKETING INC. Western Gas Marketing Limited

Northwestern Public Service Company

Port of Morgan, MT $\quad 71 \quad 1.61$

(0)

$N / A$

2.18

116

2.56

229

2.37

WESTERN GAS MARKETING INC.

Western Gas Marketing Limited

O \& R Energy

Waddington, NY

$246 \quad 2.09$

$86 \quad 2.27$

111

2.44

443

2.21

WESTERN GAS MARKETING INC. Western Gas Marketing Limited Olympic Fuels, Inc.
$161 \quad 1.61$

Port of Morgan, M
WESTERN GAS MARKETING INC. Western Gas Marketing Limited Pacific Gas \& Electric Company
Eastport, Idaho
$247 \quad 1.80$

135

1.75

543

1.73

$0 \quad N / A$

$88 \quad 2.01$

448

1.80

536

1.83 
Page - 153

SHORT-TERM SALES
4th Quarter 1993
Estimated Volumes (MMCF) \& Prices (S/MMBTU)

WESTERN GAS MARKETING INC. Western Gas Marketing Limited Pacific Gas Transmission

WESTERN GAS MARKETING INC.

Western Gas Marketing Limited Peoples Natural Gas Company

WESTERN GAS MARKETING INC. Western Gas Marketing Limited Phibro Oil \& Gas Inc.

Niagara Falls, NY $\quad 0 \quad$ N/A

Eastport, Idaho

1.65

$0 \quad N / A$

$0 \quad N / A$

N/A

$98 \quad 1.65$

WESTERN GAS MARKETING INC.

Western Gas Marketing Limited

Sierra Pacific Power Company

WESTERN GAS MARKETING INC. Western Gas Marketing Limited Southeastern Michigan Gas Company Noyes, Minnesota $31 \quad 1.77$ 31 2.05 $22 \quad 2.03$ $84 \quad 1.94$

WESTERN GAS MARKETING INC. Western Gas Marketing Limited Southwest Gas Corporation

WESTERN GAS MARKETING INC. Western Gas Marketing Limited Tarpon Gas Marketing
Waddington, NY
29

2.01

106

1.80

135

1.85
$0 \quad N / A$

$0 \quad$ N/A

93

1.80

$93 \quad 1.80$
O N/A

$25 \quad 2.27$
$0 \quad$ N/A

25

2.27 


$$
\text { Page }-154
$$

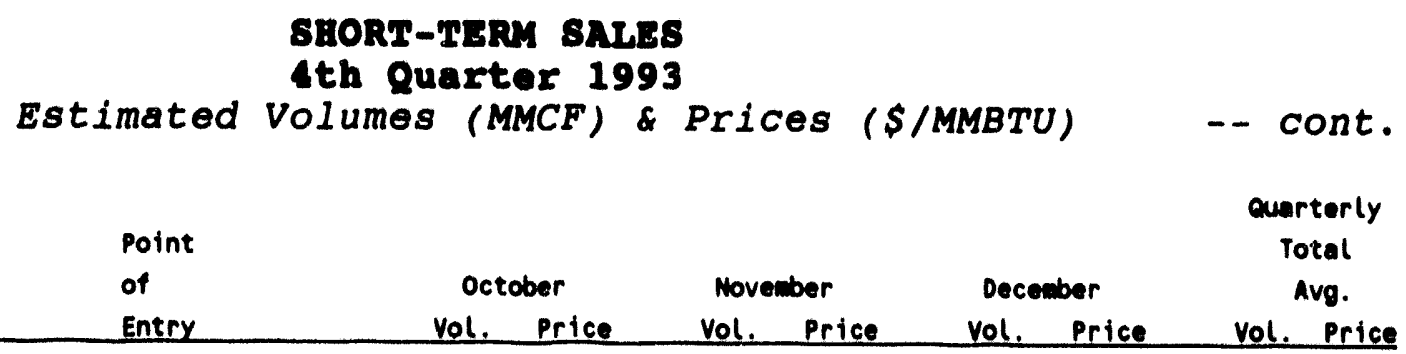

MESTERM GAS MARKETING INC.

Western Gas Marketing Liwited Tenaske Marketing Ventures

Port of Morgan, MT 109

$0 \quad N / A$

$137 \quad 1.75$

$245 \quad 1.69$

WESTERM GAS MARKETIMG INC.

Western Gas Marketing Liafted Torch Gas L.C.

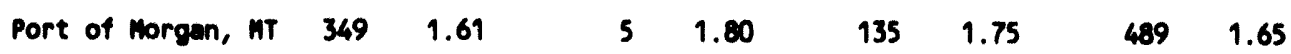

WESTERN GAS MARKETING INC.

Western Gas Marketing Limited Trensok

Port of Norgan, MT 146

1.61

O N/A

O N/A

146

1.61

MESTERN GAS MARKETING INC. Western Gas Marketing Lieited Unigas

Noyes, Minnesota

$0 \quad N / A$

$0 \quad N / A$

$68 \quad 2.03$

68

2.03

WESTERW GAS MAKETING IMC.

Western Gas Marketing Linited Western fas Marketing Inc.

Noyes, Minnesote

1.77

74

2.05

$0 \quad$ N/A

285

1.84

WESTERN GAS MARKETIMG INC. Western Gas Marketing Limited Western Gas Resources

Babb, Montana

$114 \quad 1.45$

$0 \quad N / A$

28

2.44

142

1.64

WESTERN GAS MARETING INC.

Western Gas Marketing Liaited Misconsin Matural Gas

Port'of Morgen, MT $162 \quad 1.61$

$0 \quad N / A$

O N/A

162

1.61

WESTERM GAS RESOURCES, INC. Western Gas Marketing Linited Public service of Colorado Babb, Montana $\quad 102$

$0 \quad N / A$

0 N/A

102

1.45 


$$
\text { Page - } 155
$$

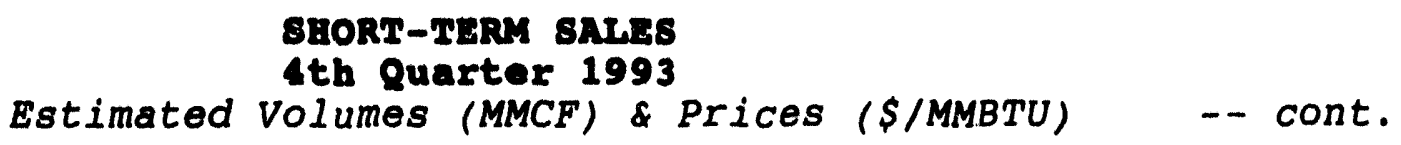

MESTERN GAS RESOURCES, INC. Western Gas Marketing Limited Washington Matural Gas Company Sumas, Mashington

WINOWARD ENERGY 2 MARKETING COMPANY Albarta 2 southern Company Ltd. Pacific Gas Electric Compeny

UISCONSIN GAS COMPANY Prosas Linited

Wisconsin Gas

UISCONSIN GAS COMPANY Western Gas Marketing Limited Hisconsin Gas 
SHORT-TERM EXPORTERS

Estimated Volumes (MMCF)

1992

1993

oct.-Dec.

Jan. - Mar

AMERICAN HUNTER EXPLORATION LTD. AMOCO ENERGY TRADING CORPORATION ANADARKO TRADING COMPANY

AQUILA SOUTHWEST MARKETING CORPORATION

BRYMORE ENERGY INC.

CANADIAN HYDROCARBONS MARKETING INC.

CATEX VITOL GAS, INC.

CENTRA GAS ONTARIO INC.

CHEVRON NATURAL GAS SERVICES, INC.

CMEX ENERGY, INC.

COASTAL GAS MARKETING COMPANY

COENERGY TRADING COMPANY

CONOCO INC.

CONSUMERS' GAS COMPANY LIMITED

CONTINENTAL ENERGY MARKETING LTO.

ENRON GAS MARKETING, INC.

INLAND GAS \& OIL CORPORATION

INTERENERGY CORPORATION

MERIDIAN OIL TRANSPORTATION INC.

MOBIL NATURAL GAS INC.

MOCK RESOURCES, INC.

NATURAL GAS CLEARINGHOUSE INC.

NORTH AMERICAN RESOURCES COMPANY

NORTH CANADIAN MARKETING CORPORATION

ORYX GAS MARKETING, L.P.

OXY USA INC.

P.M. I. COMERCIO INTERNACIONAL

PANHANDLE TRADING COMPANY

PETRO-CANADA HYDROCARBONS INC.

SANTANNA NATURAL GAS CORPORATION

ST. CLAIR PIPELINES LTD.

TARPON GAS MARKETING LTO.

TENNGASCO CORPORATION

TENNESSEE GAS PIPELINE COMPANY

TEXACO GAS MARKETING INC.

UMC PETROLEUM CORPORATION

UNION GAS LIMITED

UNION PACIFIC FUELS, INC.

VALERO INDUSTRIAL GAS, L.P.

VICTORIA GAS CORPORATION

UNIGAS ENERGY, INC.

WESTCOAST GAS SERVICES INC.

WESTERN GAS MARKETING INC.

\begin{tabular}{|c|c|c|c|c|}
\hline 0 & 73 & 0 & 290 & 0 \\
\hline 2546 & 2578 & 2966 & 4982 & 2545 \\
\hline 0 & 155 & 0 & 100 & 0 \\
\hline 1380 & 905 & 915 & 131 & 0 \\
\hline 0 & 0 & 0 & 0 & 53 \\
\hline 0 & 99 & 17 & 0 & 0 \\
\hline 0 & 0 & 0 & 100 & 0 \\
\hline 0 & 0 & 0 & 0 & 1269 \\
\hline 282 & 569 & 882 & 141 & 0 \\
\hline 752 & 0 & 0 & 0 & 0 \\
\hline 0 & 630 & 0 & 0 & 0 \\
\hline 2177 & 5848 & 793 & 0 & 464 \\
\hline 450 & 854 & 1319 & 934 & 747 \\
\hline 610 & 0 & 0 & 0 & 718 \\
\hline 40 & 24 & 0 & 0 & 0 \\
\hline 0 & 0 & 163 & 597 & 158 \\
\hline 43 & 100 & 0 & 0 & 810 \\
\hline 0 & 0 & 15 & 0 & 0 \\
\hline 1443 & 522 & 947 & 809 & 609 \\
\hline 3430 & 1353 & 1322 & 126 & 45 \\
\hline 0 & 0 & 38 & 0 & 0 \\
\hline 1076 & 0 & 335 & 0 & 0 \\
\hline 0 & 238 & 61 & 0 & 0 \\
\hline 0 & 0 & 0 & 437 & 0 \\
\hline 260 & 0 & 70 & 125 & 327 \\
\hline 0 & 111 & 0 & 0 & 0 \\
\hline 14018 & 5820 & 0 & 0 & 0 \\
\hline 0 & 210 & 0 & 50 & 0 \\
\hline 0 & 0 & 0 & 0 & 232 \\
\hline 1274 & 1603 & 1419 & 616 & 0 \\
\hline 0 & 0 & 0 & 0 & 149 \\
\hline 1078 & 0 & 0 & 0 & 0 \\
\hline 0 & 0 & 150 & 0 & 0 \\
\hline 6054 & 4613 & 5649 & 5345 & 1553 \\
\hline 623 & 715 & 900 & 79 & 0 \\
\hline 0 & 0 & 0 & 0 & 61 \\
\hline 3243 & 3111 & 3156 & 2199 & 3296 \\
\hline 0 & 0 & 0 & 100 & 0 \\
\hline 765 & 399 & 553 & 788 & 191 \\
\hline 249 & 0 & 0 & 0 & 0 \\
\hline 0 & 320 & 198 & 0 & 0 \\
\hline 7 & 755 & 220 & 0 & 0 \\
\hline 6637 & 2226 & 397 & 245 & 192 \\
\hline 48437 & 33829 & 22483 & 18194 & 13417 \\
\hline
\end{tabular}


Page - 157

PURCHASERS OF SHORT-TERM EXPORTS

Estimated Volumes Exported (MMCF)

$\begin{array}{lllll}1992 & 1993 & 1993 & 1993 & 1993\end{array}$

oct.-Dec. Jan.-Mar. Apr.-June July-Sep. Oct.-Dec.

Alberta \& Southern Gas Co. Ltd.

Altana Exploration

American Hunter Exploration Ltd.

BC Gas inc.

Canadian Hydrocarbons Marketing Inc.

Canadian Western Natural Gas

Centra Gas Marketing

Centra Gas Ontario Inc.

cibola corporation

Coastal Gas Marketing Company

Consol idated Fuel

Consumers' Gas Company Limited

Continental Energy Marketing Ltd.

Enron Gas Marketing Canada, Inc.

Ford Motor Company Canada Ltd.

Gaz Metropol itan

Gulf of Canada

Inland Natural Gas

Mobil Oil Canada

Northridge Gas Marketing Inc.

O \& R Energy

PEMEX

ProGas Limited

St. Clair Pipeline

Suncor Inc.

Tarpon Gas Marketing

Unigas Corporation

Union Gas Limited

Wes Cana Energy Marketing Inc.

Western Gas Marketing Limited

Western Gas Services

TOTALS

$\begin{array}{rr}0 & 81 \\ 0 & 238 \\ 0 & 210 \\ 43 & 100 \\ 0 & 99 \\ 40 & 0 \\ 779 & 120 \\ 0 & 0 \\ 299 & 0 \\ 0 & 0 \\ 0 & 0 \\ 922 & 70 \\ 1274 & 1627 \\ 0 & 0 \\ 31 & 0 \\ 1086 & 4373 \\ 0 & 450 \\ 0 & 0 \\ 0 & 0 \\ 0 & 506 \\ 0 & 0 \\ 27274 & 13981 \\ 1783 & 1350 \\ 0 & 0 \\ 0 & 0 \\ 778 & 33829 \\ 7 & \end{array}$

0

61

0

0

17

0

460

0

0

0

75

0

1419

0

0

285

0

38

0

15

0

10371

1365

0

0

48

220

3429

0

0

4681

22483
0

0

0

0

810

0

0

0

1269

0

232

0

1128

0

61

0

30

0

0

45

23

0

4577

0

1345

473

53

0

1626

0

0

1745

4526

13417 


\section{Page - 158 \\ SHORT-TERM SALES \\ 4th Quarter 1993 \\ Estimated Volumes (MMCF) \& Prices (S/MMBTU)}

ATTACHMENT D

\begin{tabular}{|c|c|c|c|c|c|}
\hline Exporter & Point & & & & $\begin{array}{c}\text { Quarterly } \\
\text { Total }\end{array}$ \\
\hline Purchaser/End User & Exit & Vol. Price & Vol. Price & Vol. Price & Vol. Price \\
\hline
\end{tabular}

AMOCO ENERGY TRADING CORPORATION Amoco Energy Trading Corporation PEMEX

$0 \quad N / A$

$42 \quad 2.22$

42

2.22

AMOCO ENERGY TRADING CORPORATION Amoco Energy Trading Corporation PEMEX

El Paso, Texas

$0 \quad$ N/A

$356 \quad 2.07$

$259 \quad 2.44$

614

2.23

AMOCO ENERGY TRADING CORPORATION Amoco Energy Trading Corporation PEAEX

$\begin{array}{lllllllll}\text { Hidalgo, Texas } & 1089 & 1.91 & 800 & 2.01 & 0 & \mathrm{~N} / \mathrm{A} & 1889 & 1.95\end{array}$

BRYMORE ENERGY INC.

Brywore Energy, Inc.

Gaz Metropolitan

Detroit, Michigan

$30 \quad 2.20$

$0 \quad N / A$

$0 \quad$ N/A

30

2.20

BRYMORE ENERGY INC.

Brywore Energy, Inc.

Northridge Gas Marketing Inc.

Detroit, Michigan $\quad 23 \quad 2.20$

$0 \quad$ N/A

$0 \quad N / A$

$23 \quad 2.20$

CENTRA GAS ONTARIO INC.

Coastal Gas Marketing Company

Centra Gas ontario inc.

Detroit, Michigan

$0 \quad$ N/A

624

2.73

645

2.73

1269

2.73

COENERGY TRADING COMPANY

Coenergy Trading company

Consumers' Gas Company Limited

St. Clair, MI

$2.42 \quad 302$

2.42

$0 \quad N / A$

$410 \quad 2.42$

COEKERGY TRADING COMPANY

Coenergy Trading compeny

Tarpon Gas Marketing

st. Clair, MI

$53 \quad 2.42$

O N/A

$0 \quad N / A$

53

2.42 


$$
\text { Page - } 159
$$

\section{SHORT-TERM SALES \\ 4th Quarter 1993}

Estimated Volumes (MMCF) \& Prices (\$/MMBTU) -- cont.

Exporter

Seller

Purchaser/End User

CONOCO INC.

Conoco Inc.

PEMEX

CONSUMERS' GAS COMPANY LIMITED

CoEnergy Ventures, Inc.

Consumers' Gas Company Limited

ENRON GAS MARKETING, INC.

Enron Gas Marketing, Inc.

PEMEX

INLAND GAS \& OIL CORPORATION Amoco Energy Trading Corporation $B C$ Gas Inc.

INLAND GAS \& OIL CORPORATION Conoco Inc.

BC Gas Inc.

INLANO GAS \& OIL CORPORATION Grand Valley Gas Company $B C$ Gas Inc.

INLAND GAS \& OIL CORPORATION Phillips Gas Marketing Company $B C$ Gas Inc.

INLAND GAS \& OIL CORPORATION Union Gas Limited

$B C$ Gas Inc.
Penitas, Texas

$432 \quad 1.85$

315

1.95

D N/A

747

1.89

St. Clair, MI

$108 \quad 2.17$

300

2.62

310

2.62

718

2.55

El Paso, Texas

$0 \quad N / A$

158

1.83

N/A

158

1.83

Sumas, Washington

o N/A

$0 \quad$ N/A

293

2.25

293

2.25

Sumas, Washington

$0 \quad$ N/A

$0 \quad N / A$

155

2.40

155

2.40

Sumas, Washington

$0 \quad N / A$

$67 \quad 2.36$

$0 \quad$ N/A

$67 \quad 2.36$

Sumas, Washington

$0 \quad$ N/A

o N/A

141

2.25

141

2.25

\author{
Sumas, Washington
}

N/A

- N/A

155

2.40

155

2.40 


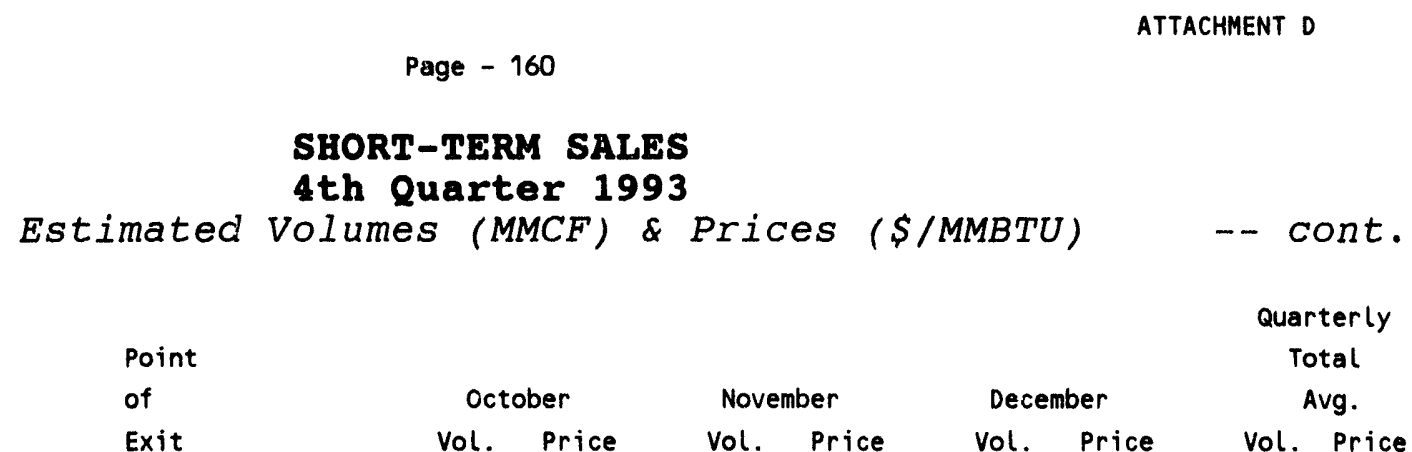

\begin{tabular}{|c|c|c|c|c|c|c|c|c|c|}
\hline Exporter & Point & & & & & & & \multicolumn{2}{|c|}{ Total } \\
\hline Seller & of & Oc & ber & Nov & per & Dec & ber & & g. \\
\hline Purchaser/End User & Exit & Vol. & Price & Vol. & Price & Vol. & Price & Vol. & Price \\
\hline
\end{tabular}

MERIDIAN OIL TRANSPORTATION INC. Meridian Oil Transportation Inc. PEMEX

El Paso, Texas

$0 \quad N / A$

$0 \quad N / A$

609

2.37

609

2.37

MOBIL NATURAI. GAS INC.

Mobil Natural Gas Inc.

Mobil Oil Canada

Harve, Montana

$0 \quad N / A$

$0 \quad N / A$

$45 \quad 2.31$

$45 \quad 2.31$

ORYX GAS MARKETING, L.P.

Oryx Gas Marketing

PEMEX

Douglas, Arizona

163

1.76

$0 \quad N / A$

164

2.14

327

1.95

PETRO-CANADA HYDROCARBONS INC.

Petro-Canada Hydrocarbons Inc.

Coastal Gas Marketing Company

Sumas, Washington

$0 \quad N / A$

$0 \quad N / A$

232

$2.6 C$

232

2.60

ST. CLAIR PIPELINES LTD.

St. Clair Pipelines

st. Clair Pipeline

St. Clair, MI

D N/A

$0 \quad N / A$

149

2.52

149

2.52

TENNESSEE GAS PIPELINE COMPANY Tennessee Gas Pipeline Company Western Gas Services

St. Clair, MI

1553

2.03

$0 \quad N / A$

$0 \quad$ N/A

1553

2.03

UMC PETROLEUM CORPORATION UMC Petroleum Corporation
Enron Gas Marketing Canada, Inc.

$0 \quad N / A$

$0 \quad N / A$

61

1.65

61

1.65

UNION GAS LIMITED

Associated Natural Gas, Inc.

Union Gas Limited

Detroit, Michigan

0

N/A

368

2.68

381

2.66

748

2.67 


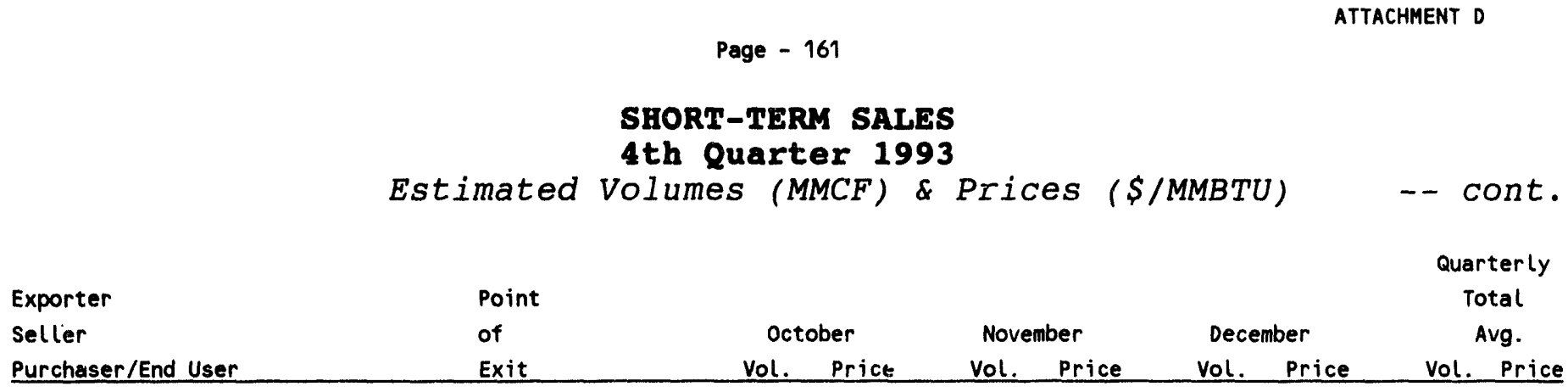

UNION GAS LIMITED

Centra Gas Ontario Inc.

st. Clair Pipeline

St. Clair, MI

311

2.30

$0 \quad$ N/A

$0 \quad N / A$

311

2.30

UNION GAS LIMITED

CoEnergy Ventures, Inc.

Union Gas Limited

St. Clair, MI

$0 \quad$ N/A

454

2.67

424

3.04

$878 \quad 2.85$

UNION GAS LIMITED

Panhandle Trading Company

st. Clair Pipeline

Detroit, Michigan

137

2.40

$0 \quad N / A$

D N/A

137

2.40

UNION GAS LIMITED

Suncor Inc.

Suncor Inc.

Detroit, Michigan $\quad 0 \quad \mathrm{~N} / \mathrm{A}$

233

2.40

241

2.43

$473 \quad 2.42$

UNION GAS LIMITED

Union Gas Limited

St. Clair Pipeline

St. Clair, MI

137

2.40

340

2.60

271

2.52

748

2.53

VALERO INDUSTRIAL GAS, L.P. Valero Industrial Gas, L.P. PEMEX

Eagle Pass, Texas

51

2.15

70

2.29

69

2.61

191

2.37

WESTERN GAS MARKETING INC. Western Gas Marketing Inc. Western Gas Services

St. Clair, MI

192

2.03

0

N/A

0

N/A

192

2.03 

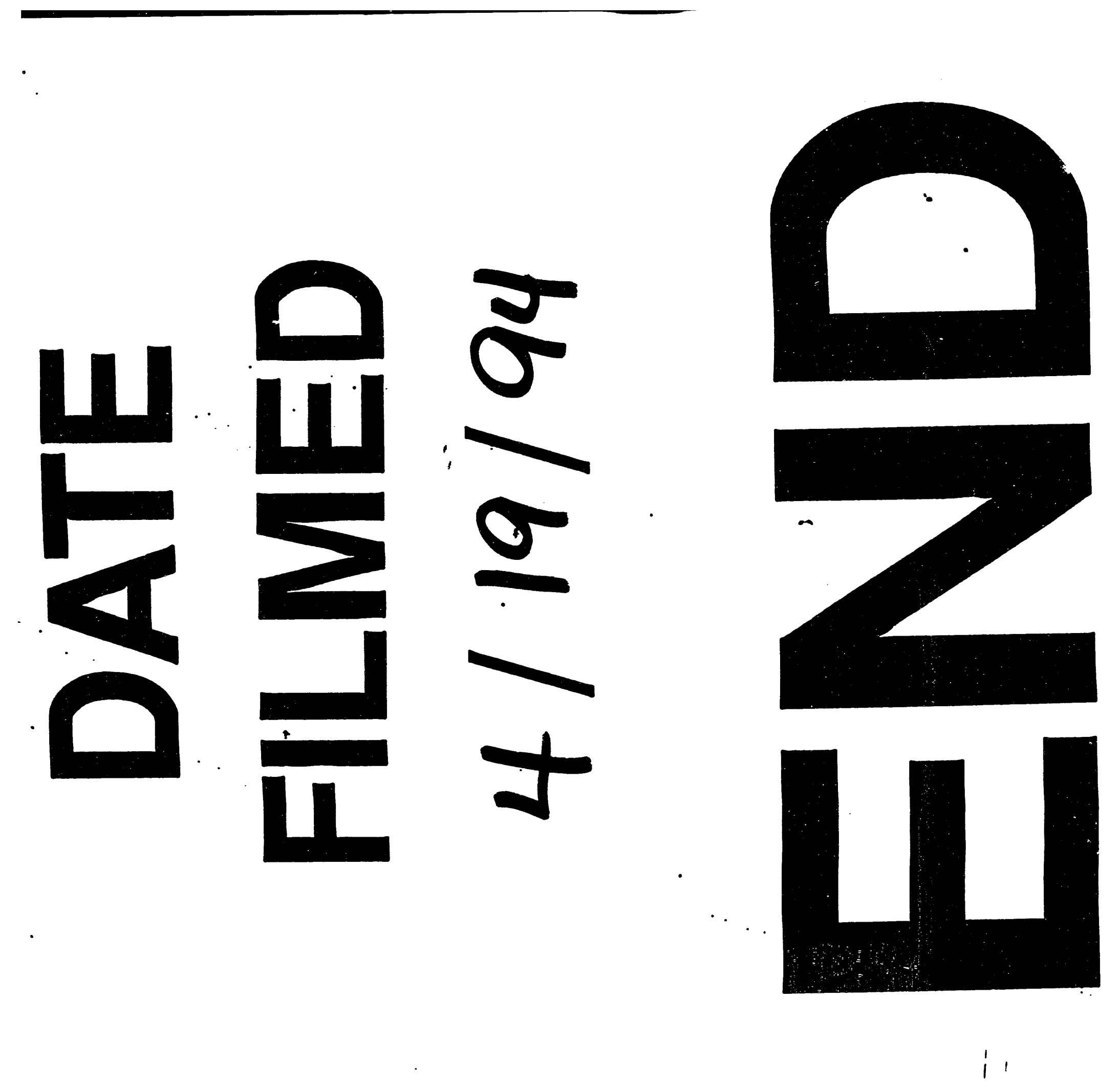


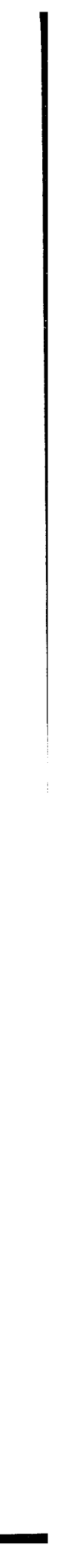

P

(20)

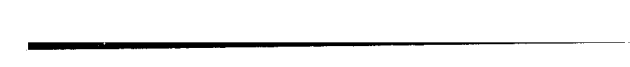

